\title{
Viktor Moser
}

\section{Chirurgen und Mechaniker auf Augenhöhe}

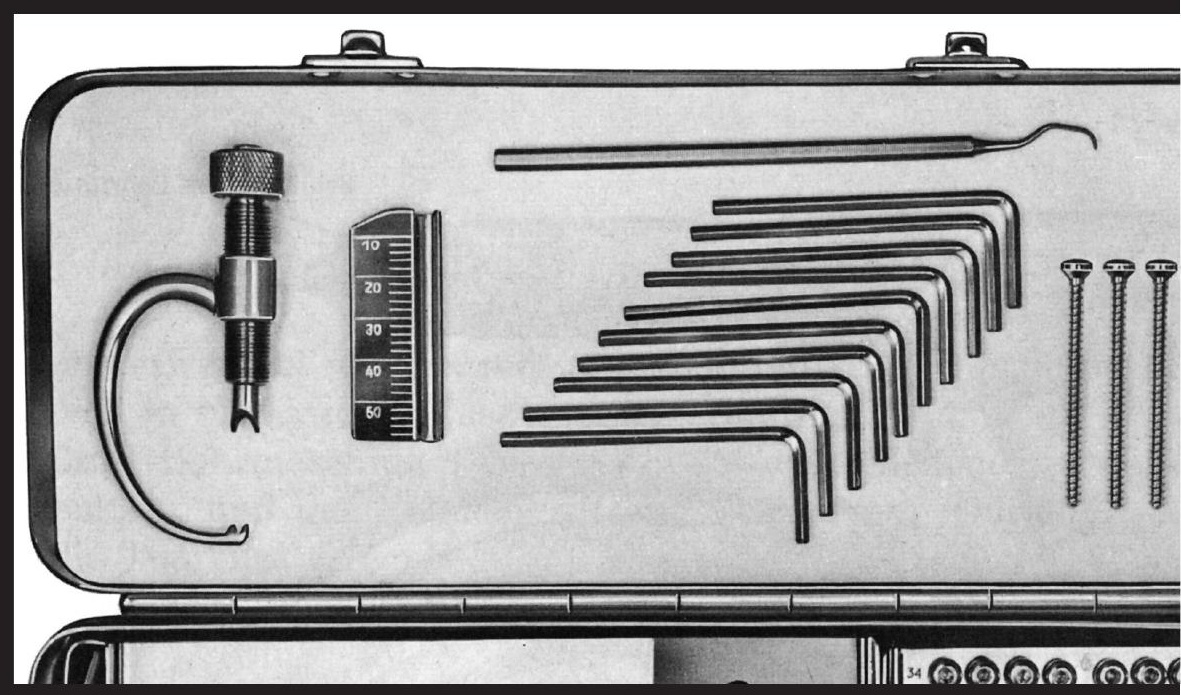

Geschichte der Medizintechnik am Jurasüdfuss 
Viktor Moser

Chirurgen und Mechaniker auf Augenhöhe 


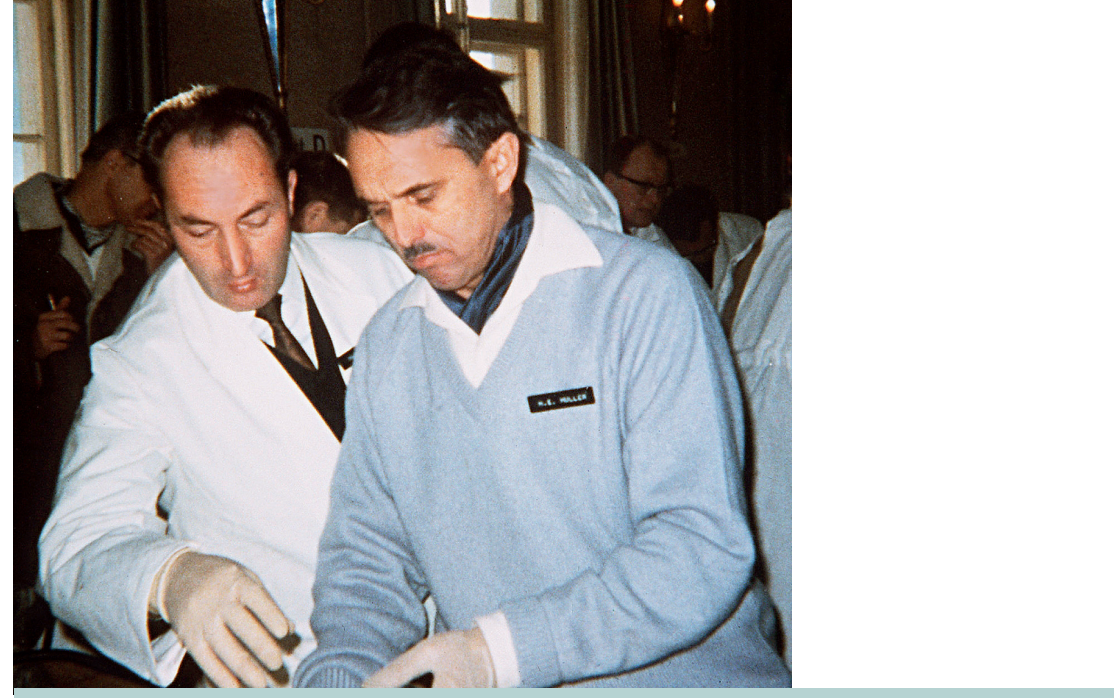

Der Mechaniker/Konstrukteur Robert Mathys sen. (links) und der Chirurg Maurice E. Müller (rechts) Copyright by AO-Foundation, Switzerland 


\section{Viktor Moser}

\section{Chirurgen und Mechaniker auf Augenhöhe}

Geschichte der Medizintechnik am Jurasüdfuss

LIBRUM Publishers \& Editors LLC 
Diese Publikation wurde gefördert durch:

\section{YPSOMED}

RMS

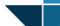

$\nabla$

\section{SWISS MEDTECH}

\section{centerVision}

sowie von Prof. Dr. Rudolf Blankart, Universität Bern,

und dem Autor, Viktor Moser, Therwil.

Lektorat: Rainer Vollmar und Henrik Halbleib, Frankfurt a. M.

Gestaltung und Satz: Katja von Ruville, Frankfurt a.M.

Druck: CPI Clausen \& Bosse, Leck

ISBN: 978-3-906897-61-5

DOI: $10.19218 / 3906897615$

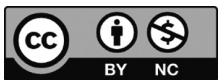

Chirurgen und Mechaniker auf Augenhöhe, Geschichte der Medizintechnik am Jurasüdfuss by Viktor Moser is licensed under a Creative Commons AttributionNonCommercial 4.o International License.

www.creativecommons.org

Open-access bei

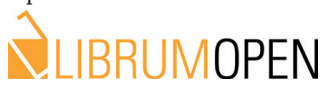

www.librumopen.com

Umschlag Vorderseite: Spritzenproduktion bei Ypsomed (Bildausschnitt) und Set mit Schrauben und Werkzeugen von Robert Mathys sen.

Umschlag Rückseite: Blutzuckermessgerät im Portfolio von Ypsomed und Robert Mathys sen. am Zeichenpult. 


\section{Inhaltsverzeichnis}

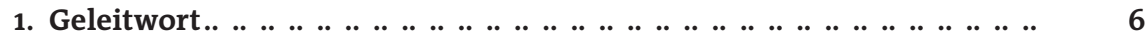

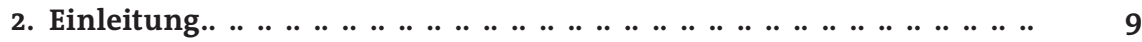

3. Was ist Medizintechnik? .. . . . . . . . . . . . . . . . . . . . . . . . . . . . . . . . . 12

4. Medizintechnik in der Schweiz - eine Erfolgsgeschichte . . . . . . . . . . $\quad 15$

5. Ein weltweiter Hotspot am Jurasüdfuss. . . . . . . . . . . . . . . . . . . . . . 25

5.1. Mathys steht am Ursprung . . . . . . . . . . . . . . . . . . . . . . . . . . . . . . . . . . . . . . 35

5.2. Synthes: Vom Aussenseiter zum Grosskonzern .. .. . . . . . . . . . . . . . 48

5.3. Stryker oder Amerika sucht die Schweiz .. . . . . . . . . . . . . . . . . . . . . . 57

5.4. Weltmarktführer Ypsomed .. . . . . . . . . . . . . . . . . . . . . . . . . . . . . . . . . . . . . . 59

6. Rahmenbedingungen und Erfolgsfaktoren . . . . . . . . . . . . . . . . . . . 66

6.1. Fruchtbarer Boden dank Uhrenindustrie . . . . . . . . . . . . . . . . . . . . . . 67

6.2. Industriell geprägte Region ... . . . . . . . . . . . . . . . . . . . . . . . . . . . . . . . . 72

6.3. Die Arbeitsgemeinschaft für Osteosynthese als Geburtshelfer und Partner . . . . . . . . . . . . . . . . . . . . . . . . . . . . . . . . . . . . . . . . . . . . . . . . . . . . . . . . . . . . . . . . . . 76

6.4. Chirurgie, Technik und Industrie arbeiten zusammen .. . . . . .. . . . . 79

6.5. Zwischen Konservatismus und Innovation . . . . . . . . . . . . . . . . . . . . 83

6.6. Kompetente Fachkräfte und gegenseitige Wertschätzung.. . . . . . . . 86

6.7. Ein Medtech-Cluster? . . . . . . . . . . . . . . . . . . . . . . . . . . . . . . . . . . . . . . . . . . . . . . . . . . . . . 93

7. Perspektiven der Medizintechnik aus Sicht der Betroffenen ... . . .. .. .. 96

8. ... und eine Stimme von jenseits des Jura.. . . . . . . . . . . . . . . . . . . . . . . 100

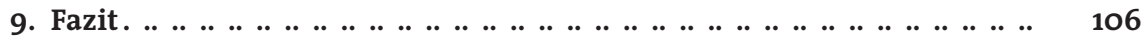

10. Anhang. . . . . . . . . . . . . . . . . . . . . . . . . . . . . . . . . . . . . . . . . . . . . . . 109

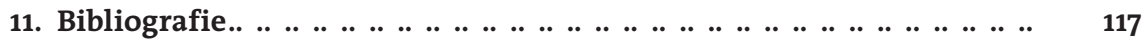

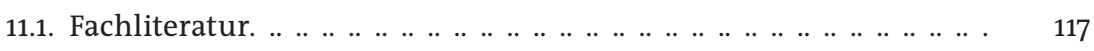

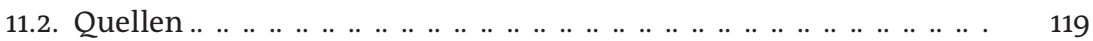

12. Persönliche Gespräche .. . . . . . . . . . . . . . . . . . . . . . . . . . . . . . . . . 122

12.1. Gesprächsprotokolle .. . . . . . . . . . . . . . . . . . . . . . . . . . . . . . . . . . . . . . 122 


\section{Geleitwort}

Was da heutzutage in den Operationssälen der Spitäler und in den Zahnkliniken alles geflickt, implantiert, korrigiert und wieder funktionsfähig gemacht wird!

Und was dabei alles an Prothesen, Implantaten, medizinischen und chirurgischen Geräten und Einrichtungen eingesetzt wird!

Und noch etwas: Was da in der Schweiz alles an medizinisch-technischen Produkten trotz hoher Löhne erzeugt und exportiert werden kann!

Heute produzieren 1400 Medtech-Firmen am Wirtschaftsstandort Schweiz. Sie beschäftigen rund 63000 meist hoch qualifizierte Mitarbeitende mit überdurchschnittlicher Arbeitsproduktivität und sie verfügen über Tausende patentfähiger Innovationen. Sie behaupten sich mit hohem Exportpotenzial auf den rasch expandierenden Weltmärkten für medizinisch-technische Spezialitäten. Bei all dem ist die ehemalige Uhrenregion am Jurasüdfuss quasi einer der Cluster, ein Ballungsraum der grössten und erfolgreichsten Medtech-Firmen.

Noch nie wurde die Wirtschaftsgeschichte der relativ jungen und rasch wachsenden Medizinaltechnikbranche firmenübergreifend aufgezeichnet. Dieses Buch ist in diesem Sinne erstmalig und einmalig. Es basiert nicht allein auf papierenen Recherchen eines Stubengelehrten, sondern kommt von einem Historiker, der nach vierzigjähriger Berufspraxis als Fachmann für innerbetriebliche Schulung und berufliche Reintegration sowie als Redaktor einer Fachzeitschrift den Arbeitsmarkt und die berufsorientierte Bildung in allen Facetten kennt. Der Autor Viktor Moser vereinigt hier die wirtschaftshistorische Sicht mit seinen langjährigen Erfahrungen mit dem Berufsbildungssystem, mit der betrieblichen Sozialpartnerschaft und mit den Standortfaktoren der schweizerischen Wirtschaft.

Das Besondere an dieser Branchenentwicklungsgeschichte liegt auch darin, dass sie im Sinne einer Oral History teilweise auf Gesprächen mit seinerzeitigen Akteuren, mit Firmengründern und ihren direkten Nachkommen beruht. Aufgrund von Firmenarchiven und Gesprächen sind hier vier Fallstudien von heute bedeutsamen Medtech-Gründergeschichten entstanden (Mathys, Synthes, Stryker, Ypsomed). Die Beschreibung markanter Episoden der Branchengeschichte machen das Buch für die Leserschaft spannend - wie etwa diese:

Wie der Starchirurg Maurice E. Müller mit dem Mechaniker und Tüftler Robert Mathys zusammenarbeitete und vorankam.

Wie Robert Mathys und Fritz Straumann im Bahnhofbuffet Olten den Weltmarkt für ihre Prothesen und Implantate quasi nach dem Zufallsprinzip aufteilten und so die Bildung von Firmenimperien vorspurten. 
Wie die Arbeitsgemeinschaft für Osteosynthese (AO) über 200000 Chirurgen und über 100 ooo Operationsfachleute aus aller Welt ausbildete und die Produzenten Mathys und Straumann auf diese Weise mit ihren Schrauben und Platten zur Vorherrschaft im Traumatologie-Weltmarkt gelangten.

Warum heute weltgrösste amerikanische Medizinalkonzerne wie Stryker trotz hoher Löhne und teurer Rahmenbedingungen in der Schweiz produzieren.

Die Medtech-Branche ist das Resultat einer einzigartigen Zusammenarbeit zwischen Chirurgen und gelernten Tüftlerhandwerkern. Keiner von beiden Berufstypen dominierte. Der eine lernte vom andern. Der Handwerker wohnte als Zuschauer im Operationssaal den chirurgischen Eingriffen bei; und der Medizinprofessor ging zum Handwerker in die Werkstatt, um die Materialtechnologie und die Prothesenbearbeitung zu entdecken.

Diese hierarchiefreie Zusammenarbeit zwischen Wissenschaftler und Handwerker ist quasi ein Alleinstellungsmerkmal des Industriestandorts Schweiz. In anderen Industriebranchen der Schweiz funktioniert die Zusammenarbeit zwischen Ingenieur, Konstrukteur und Polymechaniker ebenso fruchtbar. Oder andernorts läuft sie zwischen Chemiker und Laborant ähnlich erfolgreich ab. Für industrielle Innovationen braucht es immer beide, die Entwickler und die praktischen Handwerker.

In Deutschland mit dem vorherrschenden Akademikerdünkel, so berichten Ärzte und Ingenieure immer wieder, wäre eine solche interaktive und hierarchiefreie Entwicklungszusammenarbeit kaum denkbar. Ebenso wenig in Frankreich mit dem sehr theorielastigen Ausbildungssystem, das keine Berufslehre kennt. Auch nicht in den USA mit dem dort akuten Mangel an Skilled Workforce, also an spezialisierten Facharbeitern, die selber die Prototypen herstellen können.

Dass der Jurasüdfuss zu einem Branchencluster der Medtech-Industrie geworden ist, ist nicht zufällig. Die Vorläufer waren Dutzende von Uhrenherstellern, die schon immer Präzision, Exaktheit, Zuverlässigkeit und metallurgische Fachkenntnisse als hohe Branchenkultur gepflegt und ihre Lehrlingsausbildung danach ausgerichtet hatten.

Auch die Branchenpraxis der innerbetrieblichen Weiterbildung und die branchennahe höhere Berufsqualifikation in Fachhochschulen und höheren Fachschulen sind von der Uhrenbranche in die Medizinalepoche übernommen worden. Heute verfügt die Medizinalbranche mit ihrer hohen Innovationskraft und der dauernden Personalqualifizierung über viele tausend Patente.

Gerade am Jurasüdfuss war zudem die innerbetriebliche Sozialpartnerschaft zwischen Gewerkschaften, Betriebskommissionen und Unternehmerpersönlichkeiten hervorragend. Diese gegenseitige Akzeptanz wurde zum entscheidenden Faktor für die soziale Stabilität und Solidität der Branchen.

Der Autor zeigt auch die riskanten Gegenwartstrends der Medtech-Industrie 
auf: Welche Weltmarktentwicklungen auf die Branche einwirken, wie die Globalisierung und Kapitalkonzentration zu grossen marktmächtigen Playern führt oder wie die Regulierung und die Haftungs- und Sicherheitsanforderungen der EU der Branche zusetzen. Hier findet sich quasi eine Katalogisierung von Standortfaktoren und Erfolgsdeterminanten, aber ebenso von zukünftigen Herausforderungen und Bedrohungen der Industriekultur.

Dieses Buch ist aus dem stark bearbeiteten Fundus einer Masterarbeit des $\mathrm{Au}$ tors Viktor Moser in Applied History entstanden. Sie wurde von Professor Tobias Straumann am Historischen Seminar der Universität Zürich betreut und entgegengenommen.

Was mich an diesem Buch begeistert und beeindruckt, ist die vielseitige Sichtweise der Wirtschaftsgeschichte: Sie beleuchtet die Bedeutung der wissenschaftlichen Forschung, den Wert der Berufsbildung, die Rolle der Sozialpartnerschaft und die Innovationskultur mit den Marktentwicklungen in einer ganzheitlichen Optik und erst noch in einer spannenden Schreibweise.

\section{Rudolf H. Strahm}

Dr.h.c., Nationalökonom und Chemiker

Ehemaliger Preisüberwacher, alt Nationalrat 


\section{Einleitung}

In der von der Uhrenindustrie geprägten Region zwischen Grenchen und Solothurn ist in der zweiten Hälfte des 20.Jahrhunderts mit der Medizintechnik ein neuer Schwerpunkt entstanden. Peter Biedermann, Generalmanager von Swiss Medtech, der Dachorganisation medizintechnischer Unternehmen in der Schweiz, bringt diesen Sachverhalt auf den Punkt: «Solothurn entwickelte sich in den letzten Jahrzehnten zu einem nationalen Medtech-Hotspot, der in seiner Dichte einmalig ist.» ${ }^{1}$ Der Kanton Solothurn zählt 33 Standorte der Medizintechnik mit 92 Unternehmen (inkl. Zulieferer und Händler) mit rund 6200 Beschäftigten. Diese Zahl entspricht ziemlich genau 10 Prozent der Medizintechnikarbeitsplätze in der Schweiz. ${ }^{2}$

Die überwiegende Mehrheit der solothurnischen Medizintechnikfirmen und ihrer Zulieferer befindet sich am Jurasüdfuss. Wenn nachfolgend die Bezeichnung "Jurasüdfuss» benutzt wird, verstehen wir darunter den Raum Grenchen-Solothurn.

Ausgangspunkt unserer Studie ist folgende These: Wichtiger Erfolgsfaktor für die Entstehung und Entwicklung der Medizintechnik ist das seit Jahrzehnten verankerte Wissen und Können der Fachkräfte am Jurasüdfuss. Wesentlich zum Erfolg beigetragen haben auch die Industrietradition und die stabilen Beziehungen zwischen Arbeitgebern und Arbeitnehmern in dieser Region. Auf dieser Basis ist ein neues Cluster entstanden.

Bevor wir den Fokus auf die Entstehung und Entwicklung der Medtech-Branche am Jurasüdfuss richten, gilt es abzuklären, was zur Medizintechnik gehört und welche Merkmale sie aufweist. Das Wichtigste vorweg: Sie ist kein klar definierter Wirtschaftszweig. Medizintechnik wird etwa auch beim Bundesamt für Statistik (BfS) nicht als solche wahrgenommen. Vielmehr handelt es sich um eine Querschnittsbranche, die Physik, Chemie, Medizin- und Ingenieurwissenschaften vereinigt. ${ }^{3}$ Aus diesem Grunde ist es schwierig, genaue Zahlen über Beschäftigung oder Wertschöpfung zu erhalten; dies umso mehr, als die Zulieferbetriebe in der Produktion eine wichtige Rolle wahrnehmen. Diese stellen jedoch auch für andere Abnehmer wie z.B. die Uhren- oder Automobilindustrie Teile her. Genauere Statistiken, die diese Faktoren berücksichtigen, finden sich in den seit 2008 zweijährlich erschei-

\footnotetext{
Zit. nach: Graber, MedTech-Landschaft Kanton Solothurn, S. 2.

2 Wirtschaftsförderung Kanton Solothurn, Solothuner Life-Science-Industrie 2018, S.11.

Dümmler, Wissensbasierte Cluster in der Schweiz, S.134-136.
} 
nenden Branchenstudien von SwissMedtech und für den Kanton Solothurn seit 2016 in den Vollerhebungen der kantonalen Wirtschaftsförderung.

Die Entwicklung der Medizintechnik in der Region Grenchen-Solothurn ist Teil eines gesamtschweizerischen Prozesses. Aus diesem Grunde liefern wir einen historischen Abriss über die Entstehungsbedingungen und den Durchbruch der Medizintechnik in der Schweiz. Nach der Darstellung der sozioökonomischen und industriellen Grundlagen am Jurasüdfuss folgt ein geraffter Überblick über Entstehung und Ausbau der Medizintechnik in dieser Region. Die Ausführungen werden exemplarisch vertieft anhand der vier Unternehmen Mathys, Synthes (heute De Puy Synthes innerhalb von Johnson \& Johnson), Stryker und Ypsomed. Diese Betriebe nehmen innerhalb des Medtech-Spektrums im Kanton Solothurn einen zentralen Platz ein; nicht zuletzt deshalb, weil sie zahlreiche Zulieferfirmen beschäftigen. Zudem sind sie weltweit präsent.

Im Anschluss an den ereignisgeschichtlichen Teil analysieren wir die Rahmenbedingungen und Ursachen der Erfolgsgeschichte. Als Erstes gilt das Augenmerk den Beziehungen zwischen Uhrenindustrie und Medizintechnik. Tatsächlich sind mehrere Zentren der schweizerischen Medizintechnik in typischen Uhrenregionen entstanden. Die Verwandtschaft dieser beiden Sektoren hervorzuheben, ist beinahe schon zum Allgemeinplatz geworden. Die ehemalige Solothurner Regierungsrätin Esther Gassler hält stellvertretend für viele andere Beteiligte fest: «So kommt es denn nicht von ungefähr, dass die MedTech-Branche auch im Kanton Solothurn Fuss gefasst hat. Die von der Uhrenindustrie geprägte Meisterhaftigkeit im Umgang mit den anspruchsvollen Werkstoffen Metall und Kunststoff bis in mikroskopische Dimensionen, gepaart mit ausgesprochen innovativem Unternehmergeist, grosser Leistungsfähigkeit und hohem Qualitätsbewusstsein, bilden ideale Voraussetzungen für die MedTech-Industrie.»4

Neben der Nähe zur Uhrenindustrie ist die Region am Jurasüdfuss mit ihrer KMU-geprägten, übersichtlichen Struktur und ihrer industriellen Tradition ganz allgemein ein fruchtbarer Nährboden für die Medizintechnik. Darüber hinaus sind weitere Faktoren entscheidend. Als Erstes ist die 1958 beginnende Zusammenarbeit der Arbeitsgemeinschaft für Osteosynthese (AO) mit der Bettlacher Firma Robert Mathys zu nennen - die eigentliche Geburtsstunde. Die AO, eine Vereinigung von Schweizer Chirurgen, hatte bei der Heilung von Knochenbrüchen eine neue Technik entwickelt, wofür sie angepasste Platten und Schrauben benötigte. Aus dieser ersten Kooperation entstand über Standesgrenzen hinweg ein erfolgreiches $\mathrm{Zu}$ sammenwirken. Voraussetzung dafür waren eine hohe Innovationsbereitschaft,

4 Gassler, Esther: Die Bedeutung der medizintechnischen Firmen und von Mathys als Arbeitgeber im Kanton Solothurn, in: RMS Foundation (Hrsg.), Engagement in zweiter Generation, S. 75-76. 
mikromechanische Fähigkeiten der Fachkräfte, gegenseitige Loyalität zwischen Arbeitgebern und Arbeitnehmern sowie, daraus wachsend, eine ausgeprägte Identifikation aller Beteiligten mit der Tätigkeit und dem Unternehmen. Diese Faktoren sind nicht einzeln massgebend für den Erfolg, wohl aber in ihrer Kombination. Vor diesem Hintergrund stellt sich die Frage, ob am Jurasüdfuss von einem Medtech-Cluster gesprochen werden kann.

Danach äussern sich externe Fachleute und Protagonisten aus der Region über die Zukunftsaussichten der Medizintechnik angesichts verschärfter Regulierungen und des Spardrucks im Gesundheitswesen. Im Sinne einer Ergänzung und regionalen Erweiterung publizieren wir das Interview mit einem Medtech-Insider, der jenseits des Jura zwei Konzerne aufgebaut hat. Im Vordergrund stehen dabei die Fragen, ob die für den Jurasüdfuss gültigen Erfolgsfaktoren für die gesamtschweizerische Medizintechnik zutreffen und wieweit die eher düsteren Zukunftserwartungen aus dieser Region andernorts geteilt werden.

Unser Buch stellt die Betriebe der Medizintechnik am Jurasüdfuss ins Zentrum. Diese Sichtweise ist neu. Bisherige Publikationen zu dieser Thematik befassen sich primär mit der AO (Heim, Schlich, Jeannet) oder erwähnen die Medtech-Region im Rahmen einer Gesamtübersicht (Breiding/Schwarz oder Betschon, F. / Betschon, S. / Lindecker / Schlachter). Neu sind auch die Gespräche mit Protagonisten wie Robert Mathys jun., Robert Frigg, Thomas Wahl, Simon Michel. Auf diese Weise wurde die Quellenbasis quantitativ und qualitativ erweitert. Die Gesprächsprotokolle finden sich im Anhang. 


\section{Was ist Medizintechnik?}

Die Medizintechnik ist eine «heterogene Branche mit Querschnittcharakter». ${ }^{5}$ In amtlichen Unternehmensstatistiken umfasst sie die Herstellung von medizinischen und chirurgischen Geräten, orthopädischen Erzeugnissen, Behindertenfahrzeugen, Brillen, zahntechnischen Laboren, medizintechnischen Dienstleistungen sowie den Gross- und Detailhandel mit medizinischen, chirurgischen und orthopädischen Artikeln. ${ }^{6}$ Innerhalb des Life-Science-Sektors grenzt sie sich von der Pharmazie und der Biotechnologie ab. Sie zeichnet sich durch eine überdurchschnittliche Arbeitsproduktivität, eine ausgeprägte Exportorientierung und hohe Ansprüche an die Produktsicherheit aus.

Die betriebliche Struktur ist gekennzeichnet durch viele kleinere und mittlere Betriebe, namentlich bei den medizinischen Dienstleistungen. Diese KMU nehmen meistens die Funktion als Zulieferer wahr, unter anderem bei der Produktfertigung von kleinen Stückzahlen. Sie haben sich im Laufe der letzten Jahrzehnte von blossen Produzenten von Teilen mehr und mehr zu innovativen Erfindern entwickelt, die ihr Wissen in Form von Lizenzen an Grossunternehmen verkaufen. ${ }^{7}$ In der Schweiz arbeiteten 2008 innerhalb der Medtech-Branche 59 Prozent der Beschäftigten bei den Herstellern, gefolgt von Grosshandel und Vertrieb sowie den Zulieferern mit je 14 Prozent. Laut einer Umfrage aus demselben Jahr konzentrierten sich 63 Prozent der Beschäftigten auf 6 Prozent der Unternehmen. In den Betrieben mit weniger als zehn Mitarbeitenden - sie repräsentierten 47 Prozent aller Firmen - waren nur 3 Prozent der Arbeitskräfte tätig, weitere 12 Prozent fanden sich in Unternehmen mit 10 bis 49 und 22 Prozent in solchen mit 50 bis 249 Arbeitnehmenden. Durchschnittlich waren neunzig Personen pro Betrieb angestellt. ${ }^{8}$

Bezüglich der Qualifikationen ist die Medizintechnik eine Verbindung zwischen technischem Wissen, technischem Know-how und handwerklichem Geschick. ${ }^{9}$ Der Anteil an qualifizierten Arbeitskräften ist überdurchschnittlich hoch, zumal Kenntnisse in Mechanik, Elektronik und anderen Basistechnologien von

\footnotetext{
5 Rütter/Nathani/Popp/Holzhey, Wirtschaftliche Bedeutung der Medizintechnik in der Schweiz, S.11. 
grundlegender Bedeutung sind. ${ }^{10}$ Gemäss der bereits erwähnten Umfrage waren in der Schweiz 16 Prozent der bei den Medtech-Herstellern Beschäftigten Akademiker. Der Umsatz pro Beschäftigten betrug im selben Teilsektor jährlich $486000 \mathrm{CHF}$, beim medizintechnischen Grosshandel und Vertrieb 679000 CHF. Die Arbeitsproduktivität lag mit 229000 CHF deutlich über dem schweizerischen Durchschnitt von 143000 CHF. ${ }^{11}$

Die Medizintechnik richtet sich an ein breites Spektrum von Endnutzern: Privatpersonen, Spitäler, medizinische Labors, REHA-Kliniken, Arztpraxen, Spitex, Senioren- und Pflegeheime..$^{12}$ Fachkreise sind sich weitgehend darüber einig, dass die Nachfrage angesichts der demographischen Alterung, der zunehmenden Aktivitäten von Seniorinnen und Senioren und der gesteigerten Qualitätsansprüche tendenziell wächst. Aus diesen Gründen ist die Branche teilweise unabhängig von Konjunkturzyklen und Trends. ${ }^{13}$ In ihrer Darstellung des Markts für Medizintechnik relativieren die Autoren Schmitt-Rüth, Esslinger und Schöffski allerdings das demographische Argument. Sie weisen darauf hin, dass sich der Schwerpunkt vermehrt hin zur reparativen Medizin und zur zuverlässigen, schnellen, nicht-invasiven Diagnostik bewege. Daraus folgern sie: «Die Medizintechnik wird von der demographischen Entwicklung weit weniger beeinflusst werden als vielfach angenommen.» ${ }^{14}$ Die Autorengruppe hält auch fest, dass durch eine intensivere Zusammenarbeit zwischen Herstellern und Anwendern von Medizintechnik Zeitaufwand und Kosten reduziert werden könnten: «Medizintechnik kann hier einen entscheidenden Beitrag leisten, indem sie z. B. interne Abläufe rationalisiert oder präzisere und eindeutige Diagnosen stellt und hierdurch Doppeluntersuchungen vermeiden kann. $\aleph^{15}$ Zudem liessen sich dank ihr die Spitalaufenthalte verkürzen, die Lebensqualität verbessern und die Reintegration in den Arbeitsprozess beschleunigen. ${ }^{16}$ Diese Äusserungen deuten an, dass die Branche ausgesprochen innovativ ist. So geben Schweizer Medtech-Unternehmen bis zu 30 Prozent des Umsatzes für Forschung und Entwicklung aus, wobei grössere Unternehmen anteilmässig weniger investieren. ${ }^{17}$

Ein Wesensmerkmal der Medizintechnik ist ihre Positionierung im Spannungsfeld zwischen Marktwirtschaft und Regulierung. Bereits 1958, als der Berner Chir-

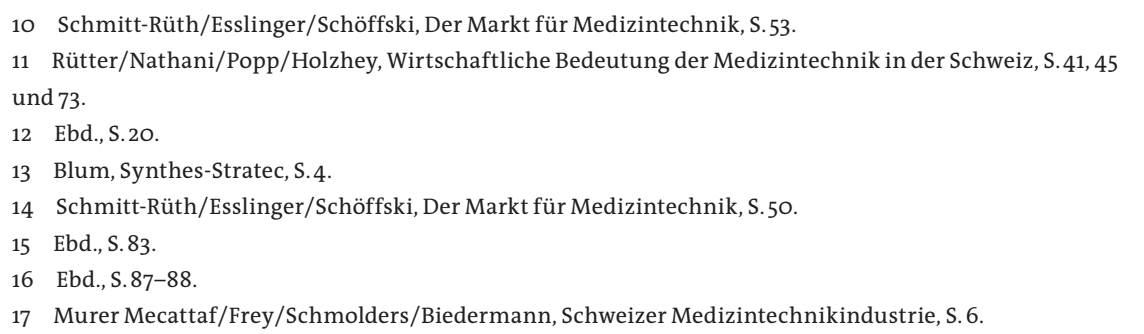


urg Maurice E. Müller und der Bettlacher Unternehmer Robert Mathys den Grundstein für die Medizintechnik am Jurasüdfuss legten, dauerte die Testphase drei bis vier Jahre. ${ }^{18}$ Diese Zeitspanne hat sich gemäss Robert Mathys jun., dem Sohn des Firmengründers, seither mindestens verdoppelt: «Die Produkteeinführung eines Implantats beträgt heute sechs bis zehn Jahre. Man muss den Markt vorbereiten, das Produkt einführen, alle Genehmigungen einholen, den Nutzen nachweisen, statistische Erhebungen durchführen, eine Dokumentation erstellen. ${ }^{19}$ Es handelt sich dabei um kostenintensive Prozesse: «In den Phasen der Markteinführung und der klinischen Akzeptanz werden etwa zwei Drittel der Gesamtkosten ausgegeben.» ${ }^{20}$ Da über 90 Prozent der schweizerischen Medtech-Produktion exportiert werden, sind die Regulierungen, etwa im Rahmen der EU, auch für die heimische Industrie verbindlich. ${ }^{21}$

18 Müller, Maurice E.: Laudatio, in: RMS Foundation (Hrsg.), Seminar zum 75.Geburtstag von Robert Mathys, S.161.

19 Gespräch mit Robert Mathys jun.

20 Schmitt-Rüth/Esslinger/Schöffski, Der Markt für Medizintechnik, S.49.

21 Rütter/Nathani/Popp/Holzhey, Wirtschaftliche Bedeutung der Medizintechnik in der Schweiz, S. 51. 


\section{Medizintechnik in der Schweiz - eine Erfolgsgeschichte}

Der Aufbau der Medizintechnik am Jurasüdfuss ist Teil einer gesamtschweizerischen Entwicklung, die sich im Wesentlichen in zwei Phasen unterteilen lässt. Der erste, zeitlich längere Abschnitt ist gekennzeichnet durch eine zusehends intensivere und systematischere Zusammenarbeit zwischen Chirurgie, Handwerk bzw. Industrie und Ingenieurwissenschaften. Daraus entstand an der Wende zum 20. Jahrhundert allmählich eine neue Branche, die auch organisatorische Veränderungen hervorrief. Anstelle punktueller philanthropischer Aktivitäten wurde das Spital der wichtigste Ort für ein immer stärker kommerzialisiertes Gesundheitswesen, wie der Historiker Pierre-Yves Donzé festhält: «Les nouvelles technologies contribuent à faire passer la médecine hospitalière d'une activité philanthropique à un business en croissance. ${ }^{22}$

Die moderne Medizintechnik ist nicht denkbar ohne die Verwissenschaftlichung der Medizin und, damit verbunden, ohne eine markante Spezialisierung ab Beginn des 19. Jahrhunderts. Hervorstechend ist dabei die Entstehung der Orthopädie als eigenständiges Fachgebiet. Es entstand ein neues Berufsfeld, das Mediziner und Chirurgen mit Handwerkern wie z. B. Bandagisten, Korsettfabrikanten und mit Verantwortlichen von Gesundheitsinstitutionen vereinigte. ${ }^{23}$ Als «Beschleuniger» wirkten bahnbrechende Erfindungen wie etwa das Stethoskop (1811), die moderne Anästhesie (1847) und neue Geräte in der Heilgymnastik. ${ }^{24}$ Die medizinischen Fortschritte generierten eine sprunghafte Nachfrage nach technischen Hilfsmitteln, die wiederum den unternehmerischen Geist vieler Handwerker weckten. ${ }^{25}$ Exemplarisch kommt der Wille zur fachübergreifenden Zusammenarbeit bei Wilhelm Conrad Röntgen zum Ausdruck. Seine Neuerfindung sollte möglichst vielen Menschen das Leben erleichtern: «Man bemühte sich ausserordentlich, die Röntgenstrahlen für den Einsatz in der Medizin nutzbar zu machen. Die Industrie arbeitete mit den Ärzten als Anwender zusammen..${ }^{26}$ Auch die Mediziner orientierten

\footnotetext{
22 Donzé, Pierre-Yves: Nouvelles technologies médicales et structuration du marché des soins à Genéve (1860-1930), in: Traverse: Zeitschrift für Geschichte, Band 19 (2012), Heft 2, Pflegekrisen, S. 33.

23 Quinn, Grégory: Jules Guérin, brève biographie d'un acteur de l'institutionalisation de l'orthopédie,

in: Gesnerus, Vol. 66.2, S. 237-240.

24 Schmitt-Rüth/Esslinger/Schöffski, Der Markt für Medizintechnik, S.14-15 und 39.

25 Breiding/Schwarz, Wirtschaftswunder Schweiz, S. 257.

26 Schmitt-Rüth/Esslinger/Schöffski, Der Markt für Medizintechnik, S. 39-40.
} 
sich aktiv hin zur Technik. Der neue Typus war "une sorte de généraliste capable, par son savoir-faire éclectique inspiré des nouvelles technologies». ${ }^{27}$

In der Schweiz wird die Klinische Orthopädie durch den Waadtländer Arzt JeanAndré Venel eingeführt. ${ }^{28}$ Als wichtigster "Gründervater» der Medizintechnik kann jedoch der Greyerzer Messerschmied Joseph-Frédéric-Benoît Charrière bezeichnet werden, der 1821 in Paris das Geschäft seines Lehrmeisters übernahm. Paris war damals das Zentrum der mit den Naturwissenschaften kooperierenden Chirurgie. Auf dieser Basis entstand eine starke Nachfrage nach chirurgischen Hilfsmitteln aller Art. Charrière stiess in diesen neuen Markt vor, baute Kontakte zu den bekanntesten Chirurgen sowie zu Armee, Spitälern und Universitäten auf. Seine Firma, die in Paris und Nogent mehr als 400 teilweise hoch spezialisierte Fachkräfte beschäftigte, produzierte Geräte und Werkzeuge vom Schoppen über den Katheter und die elastische Binde bis hin zur chirurgischen Säge, wie der Freiburger Historiker Urs Boschung betont: «Il n'est guère possible d'énumérer, même dans les grandes lignes, les instruments inventés par Charrière, scalpels, ciseaux, pinces, trousses, seringues, scies, prothèses [...] mentionnons encore ses appareils à narcose ainsi que ses équipements urologiques (sondes, cathètes) .... ${ }^{29}$ Massgebend war dabei die Entwicklung von neuen Materialien (Neusilber, rostfreier Stahl, Gummi). ${ }^{\circ}$ Parallelen zu Pionieren wie Robert Mathys, der gut hundert Jahre später mit Chirurgen kooperierte, sind unverkennbar. Nur am Rande sei erwähnt, dass die Zusammenarbeit zwischen Handwerk und Medizin zeitlich noch wesentlich weiter zurückreicht. Beim gelernten Scherer und späteren Stadtschnittarzt und Stadtarzt von Zürich Jakob Ruf (1505-1558) waren die beiden Disziplinen sogar in einer Person vereint. ${ }^{31}$

Eine stärkere Verbreitung erfuhr die schweizerische Medizintechnik im letzten Viertel des 19. Jahrhunderts. Standen bei orthopädischen Behandlungen bis anhin gymnastische Übungen im Vordergrund, wurden nach der Erfindung von medizinisch-therapeutischen Maschinen durch den Schweden Gustav Zander die Therapiemassnahmen standardisiert und rationalisiert - ein Phänomen, das sich auch in anderen medizinischen Fachbereichen wie etwa der Chirurgie verbreitete. Charles Scholder in Lausanne (1896) und Camille Reymond in Genf (1898) gründeten als Erste in der Schweiz mechanisch-heilgymnastische Institute. Die mechanischen

\footnotetext{
27 Kaba, Mariama: Jeunes filles scoliothiques et orthopédie à la Belle Époque: l'essor urbain de la mécanothérapie à Lausanne et Genève, in: Gesnerus, Vol.70.1, S.73.

28 Ebd., S. 69.

29 Boschung, Urs: Joseph-Frédéric-Benoît Charrière, fabriquant d'instruments de chirurgie à Paris,

in: Bibliothèque cantonale et universitaire, Fribourg (Hrsg.), Les Fribourgeois sur la planète, S. 52.

30 Ebd., S. 47-52.

31 Keller, Hildegard Elisabeth: Wissen und Werte verknüpft, in: Neue Zürcher Zeitung 20.2.2008.
} 


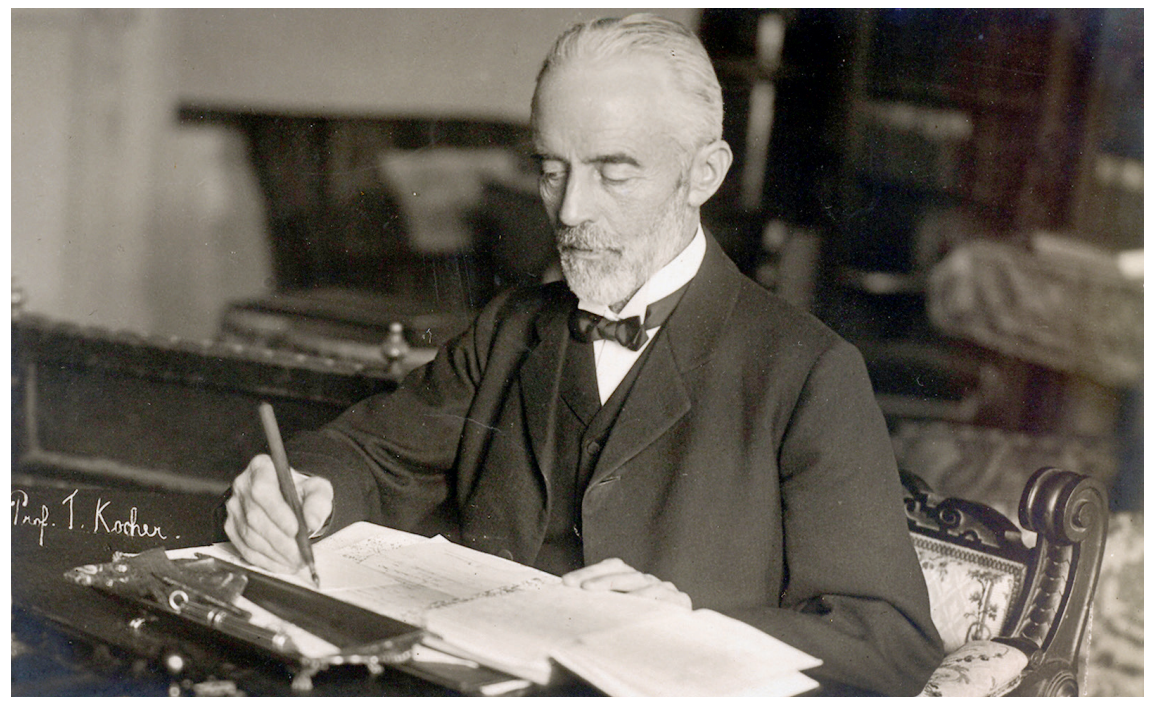

Der Chirurg Theodor Kocher 1907

Quelle: Medizinhistorisches Institut der Universität Bern

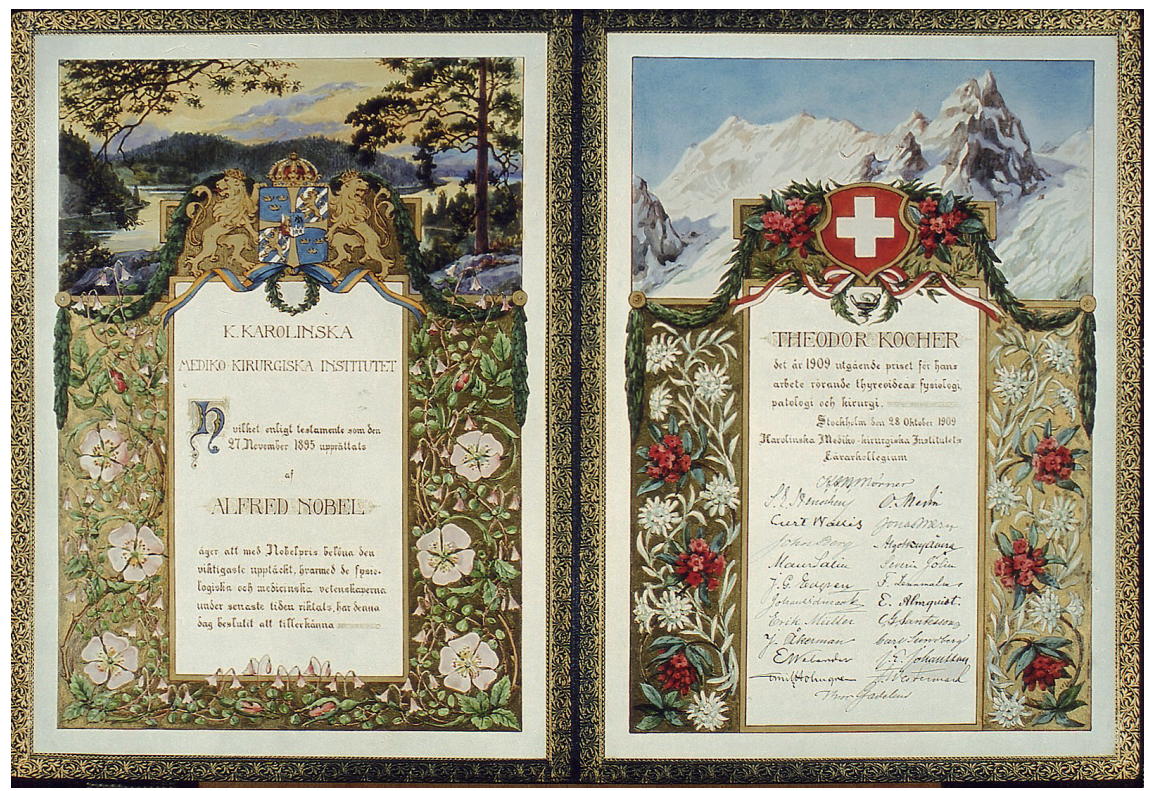

Nobelpreis 1909 für Physiologie und Medizin

Quelle: Medizinhistorisches Institut der Universität Bern 
Therapien wurden zu einer innovativen Nische auf einem sich stark entwickelnden Gesundheitsmarkt. ${ }^{32}$

Exemplarisch zeigt sich die wachsende Zusammenarbeit zwischen Chirurgie und Handwerk/Technik beim Berner Chirurgen Theodor Kocher, der 1909 den Nobelpreis für Physiologie und Medizin erhielt. Nach Kontakten mit den damals weltbekanntesten Chirurgen hatte Kocher durch zahlreiche Erfindungen die Sterblichkeit bei Operationen stark gesenkt und die Chirurgie als neue Wissenschaft weiterentwickelt. Neben einer völlig neuartigen Schilddrüsenoperation revolutionierte er durch die von ihm geschaffene Arterienklemme die Blutstillung bei Operationen. ${ }^{33}$ Der Historiker Edgar Bonjour bringt Kochers Arbeitsweise auf den Punkt: "Jede neue Operation wurde ihm zu einer neuen Theorie. Jede theoretische Deduktion brachte ihn auf neue technische Hilfsmittel für die Operation.» ${ }^{34}$ Tatsächlich interessierten ihn das Handwerklich-Technische und neue Instrumente auch auf seinen Auslandreisen stets.

Sein wohl wichtigster Ansprechpartner in Handwerkerkreisen war Maurice Schaerer, der 1892 im Berner Mattequartier die «Fabrik für Arzt- und Spitalbedarf» und 1903 das «Sanitätsgeschäft Schaerer AG» gründete. In Zusammenarbeit mit Kochers Nachfolgern produzierte die Firma später unter anderem Operationstische sowie Apparate für Sterilisation und Desinfektion. Aus ihr - das nur als Anekdote am Rande - entstand die Kaffeemaschinenfabrik M. Schaerer AG Zuchwil. ${ }^{35}$ Es ist aufschlussreich, wie die immer engere Zusammenarbeit mit der Medizin aus Sicht der Produktion erlebt wird: «Die Heilkunst hatte begonnen, sich der Technik auf vielfältigste Art zu bemächtigen, und ihr Weg in die Zukunft war nur über die Stufen ingeniöser Hilfsmittel möglich. ${ }^{36}$ Die Zusammenarbeit zwischen Theodor Kocher und Maurice Schaerer und anderen Handwerkern machte Bern bereits an der Wende zum 20. Jahrhundert zu einem Zentrum für Medizintechnik. Der Berner Chirurg Maurice Müller, der ein halbes Jahrhundert später den Kontakt zu Handwerkern vertiefte, ist demnach als Exponent Teil einer kontinuierlichen Entwicklung.

Wie bereits angedeutet, beeinflussten die technischen Umwälzungen auch das institutionelle Umfeld. Der medizinische Fortschritt konzentrierte sich auf die Spitäler, die - mehr und mehr betriebswirtschaftlich geführt - die Arbeitsteilung in der Chirurgie vorantrieben. ${ }^{37}$ Ebenso wie technologische Verbesserungen bildeten

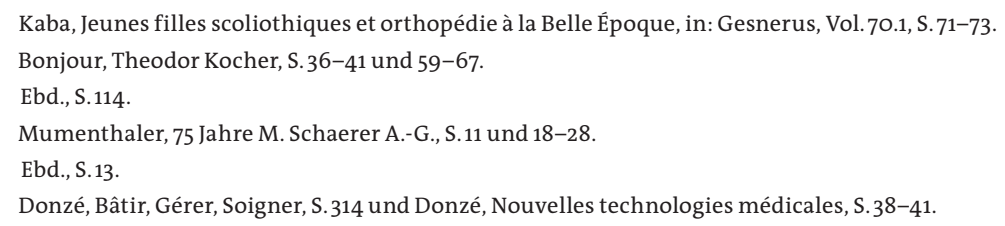




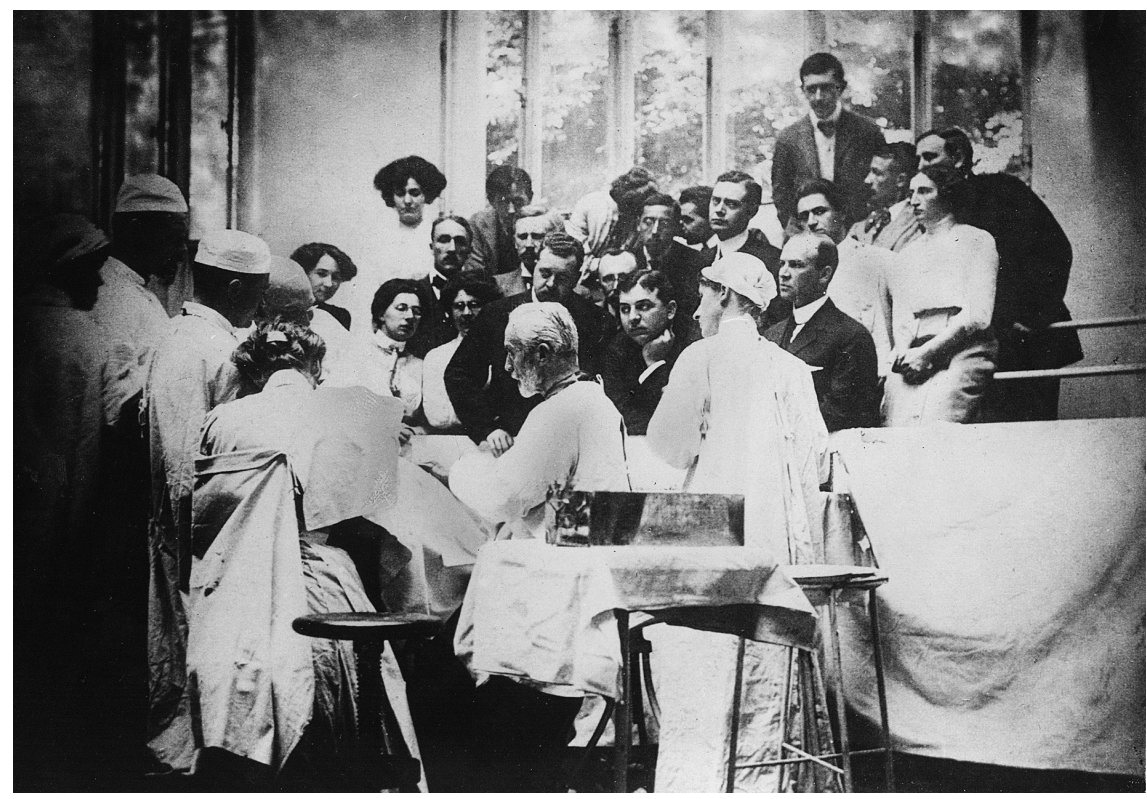

Theodor Kocher operiert vor Studenten

Quelle: Medizinhistorisches Institut der Universität Bern

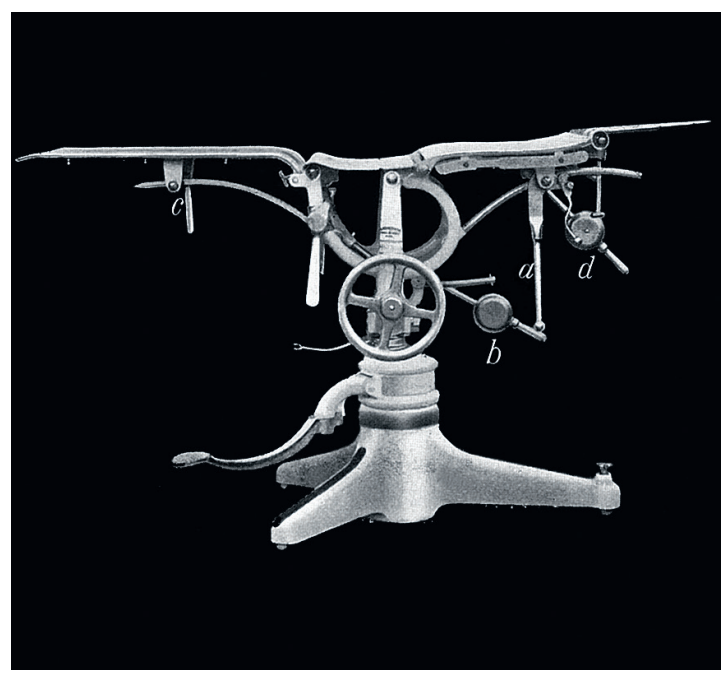

Mit diesem Operationstisch gewann die M. Schaerer AG an der Weltausstellung in Paris 1914 den "Grand Prix" (c) Schaerer Medical AG 
diese institutionellen Veränderungen eine entscheidende Basis für den Siegeszug der Medizintechnik in der Schweiz ab der Mitte des 20. Jahrhunderts.

Die zweite, zeitlich ungefähr in der Mitte des letzten Jahrhunderts einsetzende Entwicklungsperiode brachte den Durchbruch der Medizintechnik. Unsere gesamtschweizerische Übersicht behandelt die Medtech-Firmen am Jurasüdfuss bloss summarisch. Weil diese jedoch von entscheidender Bedeutung sind, lassen sie sich im Kontext nicht ausklammern. Eine ausführliche Darstellung von Mathys, Synthes, Stryker und Ypsomed findet sich in den Kapiteln 5.1., 5.2., 5.3. und 5.4.

In der Erfolgsgeschichte der schweizerischen Medizintechnik nimmt die AO (siehe Einleitung, S.10) eine zentrale Rolle ein. Statt mit Gips und Streckbettbehandlung sah sie die Heilung von Frakturen operativ mit Implantaten vor. Maurice E. Müller, Gründungsmitglied und - neben Martin Allgöwer und Hans Willenegger - treibende Kraft innerhalb der AO, hatte im April 1958 Kontakt mit Robert Mathys, Inhaber einer kleinen Maschinenfabrik im solothurnischen Bettlach, aufgenommen, um für die Heilung von Knochenbrüchen Implantate und Instrumente aus rostfreiem Stahl herstellen zu können. Mathys blieb aber nicht lange Alleinlieferant. Zwei Jahre später gelangte die AO an das 1954 gegründete Forschungsinstitut Dr.ing. Reinhard Straumann in Waldenburg (BL), um die Werkstoffe noch zu optimieren. Neben Mathys wurde Straumann zum zweiten Hersteller für Orthopädieprodukte. ${ }^{38}$ Die beiden Unternehmer hatten das exklusive Recht, die von der AO entwickelten Produkte unter dem Namen «Synthes» (in Anlehnung an Osteosynthese) zu vertreiben.

1977 stellte sich der ursprünglich aus Bern stammende Ingenieur Hansjörg Wyss an die Spitze von Synthes USA, die drei Jahre zuvor von Straumann den nordamerikanischen Markt übernommen hatte. Die immer noch von der AO gehaltene Marke Synthes verteilte sich nunmehr auf drei Player. Das Osteosynthesegeschäft des Instituts Straumann AG verselbstständigte sich 1989 unter dem Namen Stratec Medical, nachdem sich dieses durch ein Management-Buy-out von der Stammfirma gelöst hatte. ${ }^{39} 1999$ fusionierten Synthes USA und Stratec Medical zur Synthes-Stratec Inc., wo Wyss nach gut einem Jahr die Alleinherrschaft ausübte. Nachdem das neue Gebilde 2004 auch die Osteosyntheseabteilung von Mathys übernommen hatte, deckte die nun unter dem Namen Synthes Inc. firmierende Firma den gesamten Weltmarkt ab..$^{\circ}$ Das riesige und vor allem in der Unfallchirurgie weiterhin sehr

\footnotetext{
38 Breiding/Schwarz, Wirtschaftswunder Schweiz, S. 262.

39 Konrad, Thomas: Ingenieure der Medizin. Institut Straumann. Von Uhrenfedern zu Zahnimplantaten,

in: Betschon/Betschon/Lindecker/Schlachter (Hrsg.), Ingenieure bauen die Schweiz, S. 438-439.

40 Breiding/Schwarz, Wirtschaftswunder Schweiz, S. 266.
} 
erfolgreiche Unternehmen wurde am 14.Juni 2012 für 19,7 Mrd. USD an den USKonzern Johnson \& Johnson (J \& J) verkauft. ${ }^{41}$

Auf Maurice E. Müller geht ein weiteres ehemaliges Flaggschiff der Schweizer Medizintechnik zurück. Müller war nicht nur Unfallchirurg, sondern auch Orthopäde. In dieser Eigenschaft entwickelte er 1960 eine neue Hüftprothese. Da die Mathys AG allein die Nachfrage nicht erfüllen konnte, nahm er mit Sulzer Winterthur Kontakt auf. Das war der Beginn des bekannten «Sulzer-Hüftgelenks». Zusammen mit Sulzer gründete Müller 1965 die Protek AG, mit der er seine Erfindung perfektionierte. Parallel dazu entstand - ebenfalls in Winterthur - 1968 die Firma Allo Pro, die zehn Jahre später gemeinsam mit dem Wiener Professor Karl Zweymüller die erste zementfreie Hüftprothese auf den Markt brachte. Im selben Zeitraum (1970) wurde die Medizintechnik bei Sulzer zu einer eigenen Abteilung. ${ }^{22}$

Die verschiedenen Firmengründungen spiegeln einen Boom wider, der ursächlich mit Materialentwicklungen wie etwa einer neuen Titanlegierung zusammenhing. Bezüglich Körperverträglichkeit, Korrosionswiderstand und mechanischer Festigkeit waren die neuen Werkstoffe bahnbrechend. ${ }^{43}$ Es handelte sich um "reibungsarme Materialpaarungen, die schmerzfrei Gelenkbewegungen gewähren und geringstmögliche Abnützungen zeitigen».44 Wichtig waren zudem eine sofortige Funktionsfähigkeit und eine maximale Lebensdauer. Mit seinen qualitativ hochstehenden Produkten nahm Sulzer auf dem Weltmarkt eine Spitzenposition ein. Nach dem Erwerb von Mehrheitsbeteiligungen bei Protek AG und Allo Pro wurde die Firma zum grössten nicht amerikanischen Anbieter von Implantaten. 1990 erschien die Medizinabteilung unter dem neuen Namen Sulzer Medica als eigenständige Einheit innerhalb des Konzerns. Wiederum fünf Jahre später vereinigte sich diese mit Allo Pro, Protek AG und der amerikanischen Intermedic Ortopedics. 1997 folgte der Börsengang, und im folgenden Jahr verzeichnete Sulzer Medica einen Umsatz von 1,5 Mrd. CHF. Der kometenhafte Aufstieg endete abrupt. Ende 2000 und Mitte 2001 musste sich die Firma nach einer Sammelklage wegen verunreinigter Hüftgelenke und Knieimplantate vom US-Markt zurückziehen. Anfang 2002 willigte sie zu einem Vergleich in Höhe von 1 Mrd. CHF ein. 2003 wurde Sulzer Medica durch die amerikanische Zimmer Corp. übernommen. Auch wenn dadurch eine renommierte Schweizer Firma von der Bildfläche verschwand, blieb der neue Eigentümer in der Schweiz stark präsent. ${ }^{45}$

\footnotetext{
41 Müller, Giorgio V.: Synthes bleibt der Schweiz treu, in: Neue Zürcher Zeitung 14.6.2012.

42 Breiding/Schwarz, Wirtschaftswunder Schweiz, S. 262-265.

43 Buchel, André P.: Medizintechnik vom Maschinenbauer. Vier Jahrzehnte Sulzer, in: Betschon/Betschon/ Lindecker/Schlachter (Hrsg.), Ingenieure bauen die Schweiz, S. 379-383.

44 Ebd., S. 383.

45 Ebd., S. 398-407.
} 


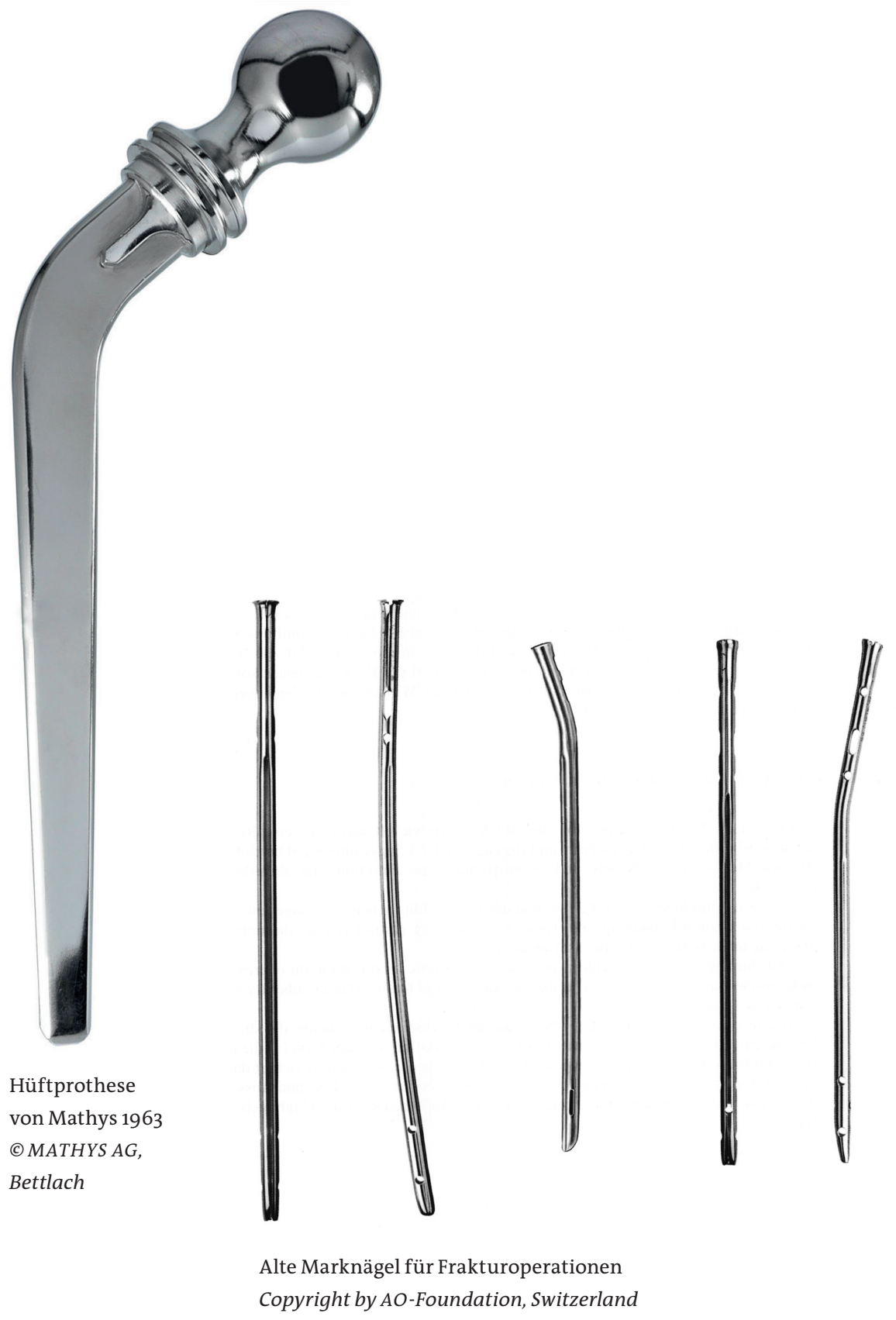


Neben der Traumatologie und der Orthopädie sind in der Schweiz auch in anderen Bereichen der Medizintechnik weltbekannte Grossbetriebe entstanden. So hat Thomas Straumann, der Enkel des Firmengründers Reinhard Straumann, nach dem Management-Buy-out der Osteosynthese-Abteilung (siehe S.20) den bereits bestehenden Sektor der Zahnprothetik ausgebaut und die Firma 1998 an die Börse gebracht. Das seit 2004 in Basel ansässige Unternehmen beschäftigt heute bei einem Jahresumsatz von knapp 1 Mrd. CHF insgesamt rund 4000 Mitarbeitende. ${ }^{46}$ In ähnliche Dimensionen stiess der Zahnimplantatehersteller Nobel Biocare vor, der 2014 vom amerikanischen Konzern Danaher übernommen wurde und in dessen Struktur er ein hohes Mass an Eigenständigkeit bewahren konnte. ${ }^{47}$

Auf dem Gebiet der Hörgeräte ist die Stäfner Firma Sonova einer der Weltmarktführer. Der 1947 als AG für Hörgeräteakustik gegründete Betrieb eroberte sich seine Spitzenposition dank kontinuierlicher technischer Neuerungen, die zusehends eine Miniaturisierung der Geräte ermöglichten. Bei einem Jahresumsatz von über 2,6 Mrd. CHF zählt der Konzern heute mehr als 14200 Beschäftigte. ${ }^{4}$

Ein wichtiges Kapitel in der Geschichte der schweizerischen Medizintechnik hat die Plus Orthopedics geschrieben. Sie entstand 2005 aus der Fusion der drei Medical-Holding-Firmen Plus Endoprotetik, Intraplant und Precision Implants. Zwei Jahre später wurde Plus Orthopedics zwar vom britischen Medizintechnikunternehmen Smith \& Nephew übernommen. Dieses verlegte jedoch seinen europäischen Hauptsitz und das globale Distributionszentrum nach Zug und behielt den Entwicklungs- und Produktionsstandort in Aarau bei. Als Beispiele für weitere international erfolgreiche Medtech-Unternehmen erwähnen wir die auf Selbstinjektionssysteme spezialisierte Ypsomed (siehe Kapitel 5.4.) und die 1980 gegründete Tecan, die einer der wichtigsten Hersteller von Laborinstrumenten ist.

Schweizer Medizintechnikfirmen sind auf der ganzen Welt tätig. Umgekehrt wählen ausländische Konzerne die Schweiz als Produktionsstandort. Neben Zimmer, J\&J, Stryker gehört dazu auch Medtronic. Dieser amerikanische Branchenriese hat in Tolochenaz (VD) nicht nur seinen Hauptsitz für Europa und Zentralasien sowie das europäische Schulungszentrum, sondern auch eine hochmoderne Fabrik für implantierbare Herz- und Hirnschrittmacher. Laut Angaben der Oberzolldirektion trug 2014 allein diese Produktionsstätte 0,55 Prozent zum schweizerischen Aussenhandel bei. ${ }^{49}$

\footnotetext{
46 www.straumann.com > Über uns > Zahlen und Fakten > Unsere Geschichte und Konrad, Thomas: Ingenieure der Medizin., in: Betschon/Betschon/Lindecker/Schlachter (Hrsg.), Ingenieure bauen die Schweiz, S. 438-443.

47 Breiding/Schwarz, Wirtschaftswunder Schweiz, S. 270.

48 Ebd., S. 257-261 und www.sonova.com > Über uns > Fakten und Zahlen.

49 Breiding/Schwarz, Wirtschaftswunder Schweiz, S. 266-269.
} 
Die volkswirtschaftliche Bedeutung der Medizintechnik für die Schweiz lässt sich mit Zahlen belegen. In 1350 Unternehmen beschäftigte sie 2014 rund 54500 Mitarbeitende. Das waren rund 5000 Personen mehr als zehn Jahre zuvor, was auf ein stetiges Wachstum hinweist. Der Gesamtumsatz betrug 2015 14,1 Mrd. CHF. Ihr Anteil am Bruttoinlandprodukt belief sich im selben Jahr auf 2,2 Prozent und jener an der Gesamtzahl der Beschäftigten auf 1,1 Prozent. Ebenfalls 2014 wurde ein Exportvolumen von 10,6 Mrd. CHF registriert, was 4 Prozent aller Schweizer Exporte entsprach. Diese Zahlen bilden einen mittelfristigen Trend ab. Über mehrere Jahre gesehen, zeigte die Medizintechnik ein konstantes Umsatzwachstum von jährlich 6 Prozent. ${ }^{50}$ Der Export stieg zwischen 2001 und 2008 um 109 Prozent. ${ }^{51}$ Tatsächlich konnte (und kann) der Standort Schweiz 2014 als globaler Schwerpunkt der Medizintechnik bezeichnet werden, wies er doch innerhalb Europas die grösste Dichte an medizintechnischen Unternehmen auf. Der Anteil an allen Medtech-Beschäftigten in Europa belief sich 2008 auf ungefähr 10 Prozent. ${ }^{52}$ Diese Relationen haben sich gemäss Auskunft der Dachorganisation Swiss Medtech bis heute nicht wesentlich verändert.

50 Murer Mecattaf/Frey/Schmolders/Biedermann, Schweizer Medizintechnikindustrie, S. 5, 9 und 13.

51 Rütter/Nathani/Popp/Holzhey, Wirtschaftliche Bedeutung der Medizintechnik in der Schweiz, S. 68-69.

52 FASMED, Medizintechnik - eine Schlüsselbranche, S. 6-7. 


\section{Ein weltweiter Hotspot am Jurasüdfuss}

Die Gesamtübersicht über die Geschichte der Medizintechnik am Jurasüdfuss dient als Orientierungsrahmen. Sie bleibt zunächst summarisch, da wir sie durch die exemplarische Beschreibung der vier wichtigsten Firmen konkretisieren und vertiefen werden. Dieses Vorgehen ist nicht nur methodisch, sondern auch inhaltlich begründet. Die vier ausgewählten Betriebe Mathys, Synthes, Stryker und Ypsomed decken das medizintechnische Spektrum in der Region umfassend ab. Drei von ihnen sind im Kerngebiet der Traumatologie und Orthopädie tätig, während der vierte (Ypsomed) mit der Injektionstechnik ein weiteres wesentliches Aktionsfeld der Medizintechnik bedient. Zudem lassen sich am Beispiel der vier ausgewählten Betriebe die geschichtlichen Abläufe präzis und anschaulich darstellen. "Ausgewählt» ist insofern der falsch gewählte Begriff, als sich die vier Firmen aufgrund ihrer regionalen Bedeutung gleichsam von selbst anbieten. Sie alle unterhalten ein fein verästeltes Netz an Zulieferbetrieben und gehören mit ihrer offensiven Forschung und Entwicklung weltweit nach wie vor zur Spitzengruppe der Innovatoren in der Medizintechnik.

Besondere Aufmerksamkeit gilt der AO (siehe Kapitel 6.3.). Von Anfang an hatte diese Vereinigung von Chirurgen einen entscheidenden Einfluss auf die Medizintechnik in der Region zwischen Grenchen und Solothurn und ist mit der Entwicklung dieses Industriezweiges am Jurasüdfuss untrennbar verbunden. Namentlich während der ersten fünfzig Jahre der regionalen Medizintechnik ist die AO als Vertragspartnerin der beiden Firmen Mathys und Straumann, später für den Straumann-Nachfolger Stratec Medical sowie für die 1974 gegründete Synthes USA, eine wesentliche Konstante.

Nicht immer lässt sich der Startpunkt einer Entwicklung genau datieren. Im Falle der Medizintechnik am Jurasüdfuss ist dies jedoch möglich. Ihre Geschichte beginnt am 8. April 1958 mit der bereits erwähnten Begegnung des Mechanikers und Konstrukteurs Robert Mathys mit dem Chirurgen Maurice E. Müller (siehe S. 6). Mathys, in seinem Umfeld als Tüftler bekannt ("Motörli-Röbu»), leitete zu diesem Zeitpunkt in Bettlach den 1946 von ihm gegründeten Mechanik- und Décolletagebetrieb. Zusammen mit 15 Mitarbeitern entwarf, entwickelte und produzierte er rostfreie Stahlschrauben und andere Mikroelemente, insbesondere für die nahe gelegene Uhrenindustrie. Der aus Biel stammende Chirurg Maurice E. Müller seinerseits suchte für die von ihm initiierte Bruchheilung mit Schrauben und Platten einen Fachmann, der in der Lage wäre, die benötigten Instrumente und Bestandteile in der gewünschten Qualität herzustellen. Durch die Vermittlung eines Stahl- 


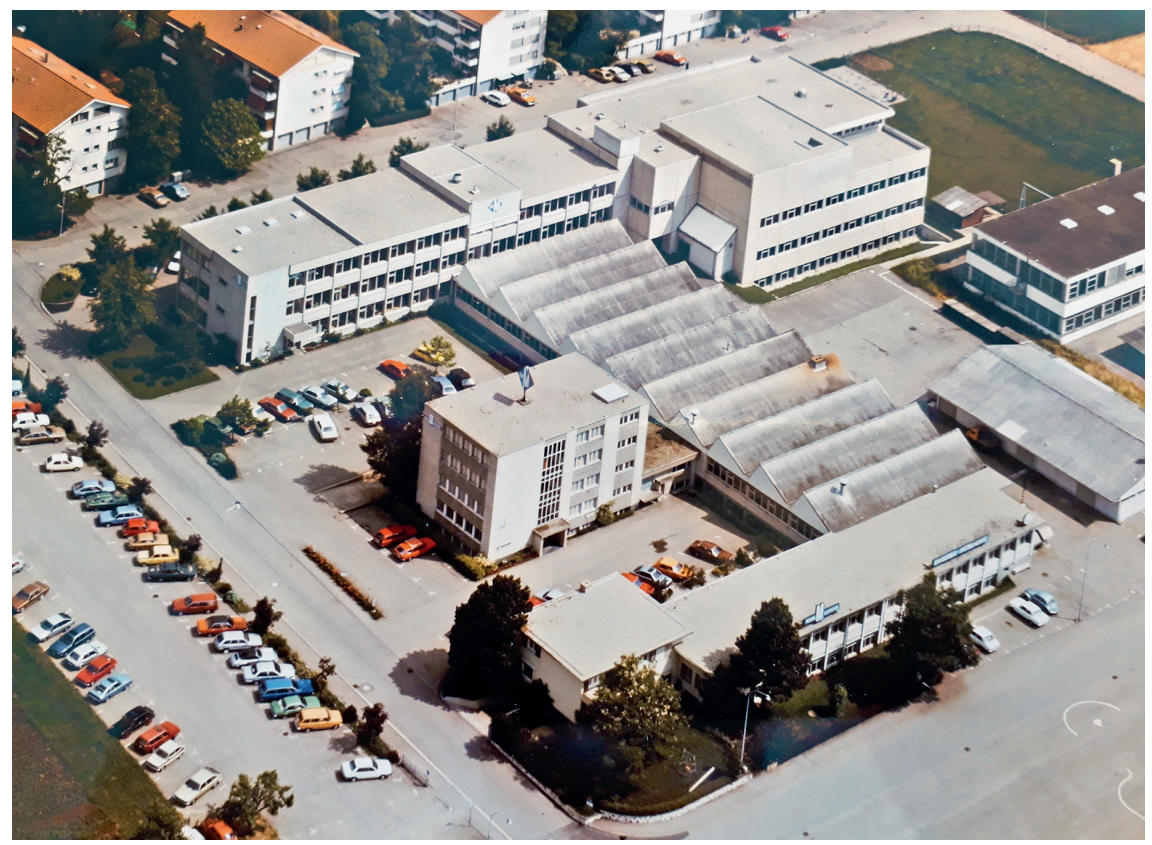

Die Firma Mathys in den 196oer-Jahren. Im Vordergrund die ältesten Gebäudeteile (c) MATHYS AG, Bettlach

lieferanten stiess er auf Robert Mathys, der sich mit viel Geschick und Engagement an die Realisierung des Auftrags machte. ${ }^{53}$ Nach einer Operationsassistenz im Spital Grenchen hatte Mathys bis zur Gründung der AO am 6. November 1958 alle Prototypen hergestellt. ${ }^{54}$

Einziger Konkurrent von Mathys wurde 1960 das Reinhard-Straumann-Forschungsinstitut in Waldenburg (BL). Dieses Unternehmen nahm, insbesondere für die Uhrenindustrie, Materialprüfungen vor. Bereits in den Jahren zuvor hatte der Firmengründer Reinhard Straumann bahnbrechend neue Legierungen für die Uhrenindustrie entwickelt. Nachdem bei den von Mathys hergestellten Implantaten erste Korrosionsschäden aufgetreten waren, kontaktierte das AO-Mitglied Hans Willenegger den ihm bekannten Reinhard Straumann. Dessen Sohn Fritz, ebenfalls Ingenieur und Leiter des Instituts, nahm mit der AO Verhandlungen auf. Zusammen mit seinem Team entwickelte er einen korrosionsfreien Stahl von bisher unerreichter Qualität.

53 Jeannet, Leading a Surgical Revolution, S. 74 .

54 Ebd., S.76. 


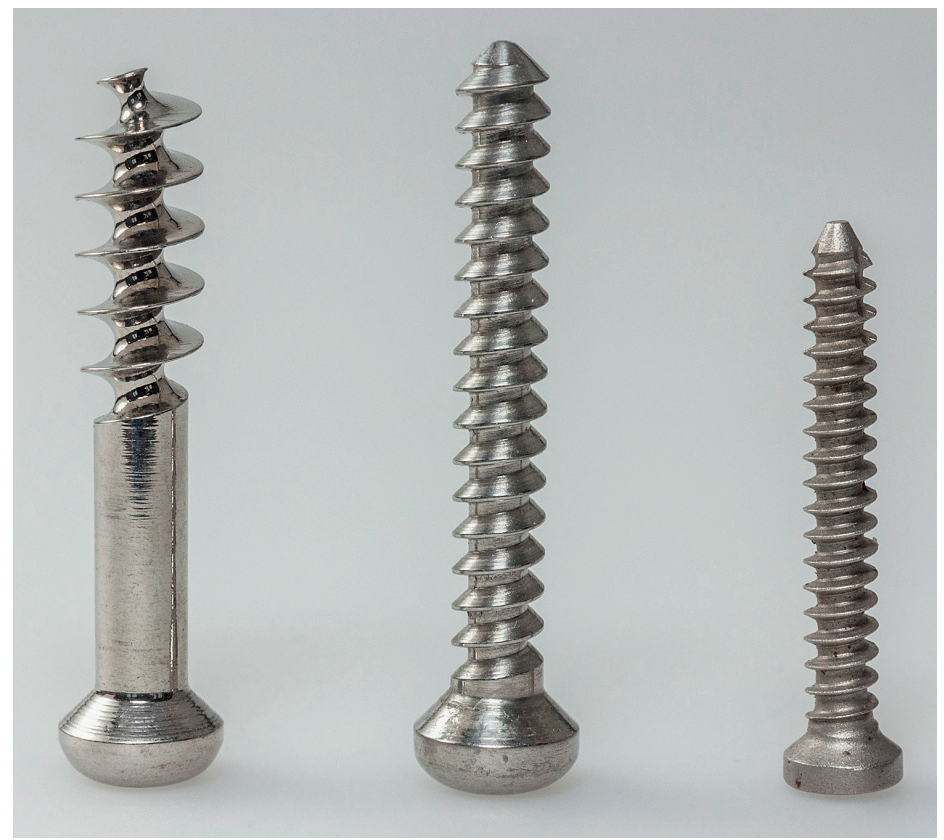

Entwicklung der Knochenbruchschrauben: System von Danis (links), System AO Kortikalischraube (Mitte), Spongiosaschraube (rechts)

Copyright by AO-Foundation, Switzerland

Am 21. November 1963 schloss die von der AO gegründete Synthes AG Chur mit Mathys und Straumann einen Vertrag ab, gemäss dem den beiden Produzenten das exklusive Recht für Produktion und Verkauf der Instrumente und Bestandteile zugestanden wurde. Die AO ihrerseits bewahrte als Patentinhaberin das alleinige Urheberrecht am gesamten Instrumentarium. Folgerichtig erhielt sie bzw. die Synthes AG Chur Lizenzgebühren, die zunächst ungefähr 15 Prozent der Produktpreise betrugen. Wörtlich wurde festgehalten: «Synthes als alleinige Inhaberin aller Rechte an den Instrumentarien nach den Prinzipien der Arbeitsgemeinschaft für Osteosynthese überträgt den Produzenten das alleinige Recht zur Herstellung und zum Vertrieb der Vertragsgegenstände ...»55

Spätestens an dieser Stelle drängt es sich auf, Entstehung, wichtige Meilensteine und Funktion der AO genauer zu betrachten, denn ohne sie gäbe es keine Medizintechnik am Jurasüdfuss. Am Ursprung der AO steht die Begegnung des Chirurgen und Orthopäden Maurice E. Müller mit Robert Danis 1950 in Brüssel. Danis war weltweit einer der ersten Chirurgen, die die Methode der Osteosynthese anwen-

55 Jeannet, Leading a Surgical Revolution, S.126-127 und zit. nach: Heim, Das Phänomen AO, S. 96. 
deten. Diese propagierte anstelle von Strecken und Immobilisierung die operative Heilung von Brüchen. Bei Danis wurde Müller auch klar, dass ein erfolgreicher Chirurg sein eigenes Werkzeug entwickeln musste.

Zusammen mit zwölf gleichgesinnten Berufskollegen gründete Müller am 6. November 1958 im Hotel Elite Biel die AO. Deren erster Obmann wurde Müllers Freund aus Bieler Zeiten Robert Schneider. Zur Kerngruppe der AO gehörten ausserdem Martin Allgöwer, Hans Willenegger und Walter Bandi. ${ }^{56}$ Als Verein, ausschliesslich bestehend aus praktizierenden Chirurgen, konstituierte sich die AO am 19. März 1960 in Chur. Das Prinzip der Osteosynthese bestand darin, dass das gebrochene Glied durch Schrauben, Platten und Nägel in seinen früheren anatomischen Zustand zurückversetzt wurde. Dafür waren eine absolute Stabilisierung des Knochens und eine kleinstmögliche Beeinträchtigung des Gewebes vonnöten. Von allem Anfang an stand eine optimale Standardisierung der Vorgehensweise im Vordergrund. Auf diesem Grundsatz basierten die vier Säulen der AO: Lehre, Forschung, Dokumentation und Instrumentarium..$^{57}$ Einheitliche Instrumente in Form eines ganzen Sets waren damals ungewohnt, für eine standardisierte Operationstechnik aber unerlässlich, wie der Medizinhistoriker Thomas Schlich betont: «The creation of the AO instrument sets was an important part of the trend toward standardisation and constituted a very elegant form of supporting and disciplining the users. ${ }^{58}$

Die Technik der Osteosynthese war zu Beginn der 196oer-Jahre noch völlig neu. Es verwundert deshalb nicht, dass die AO-Mitglieder in Chirurgenkreisen stark kritisiert und als "gefährliche Sektierer» betrachtet wurden. So schrieb Maurice E. Müller Jahre später über die feindliche Stimmung am Kongress der Schweizerischen Gesellschaft für Chirurgie vom 24. November 1960 in Bern: «But it was apparent that AO faced a hostile world, which was far from ready to accept anything we had to offer.» ${ }^{59}$ Die AO stand folglich unter starkem Legitimationsdruck. Zum Durchbruch verhalfen ihr nicht zuletzt die offensichtlich grösseren Erfolge der Osteosynthese gegenüber traditionellen Techniken. Ihre Überlegenheit wurde eklatant, wenn man bedenkt, dass gemäss der Schweizerischen Unfallversicherungsanstalt noch unmittelbar nach Kriegsende 40 Prozent der Versicherten im Anschluss an Tibiafrakturen und 70 Prozent nach Femurfrakturen eine Dauerrente erhielten. ${ }^{60}$

Neun Monate nach der statutarischen Vereinsbildung gründete die AO am 10. Dezember 1960 die Synthes AG Chur mit der Absicht, gegenüber den Produzen-

\footnotetext{
56 Heim, Das Phänomen AO, S. 33-38.

57 Ebd., S. 64-67 und 70-71.

58 Schlich, Surgery, Science and Industry, S. 52.

59 Jeannet, Leading a Surgical Revolution, S.109.

60 Heim, Das Phänomen AO, S. 26.
} 
ten Mathys und Straumann als geschäftlicher Ansprechpartner aufzutreten. Bis zum bereits erwähnten Vertrag dauerte es dann nochmals rund drei Jahre. Von diesem Zeitpunkt an war die Geschichte der AO eng mit den beiden Herstellern verknüpft. Dies zeigte sich besonders deutlich, als sich im Laufe der ersten Jahre die Konkurrenz auf internationaler Ebene merklich verschärfte. Als Beispiel seien die Geschäftspraktiken der 1963 von den ehemaligen Straumann-Ingenieuren Vogt und Karpf errichteten Firma Osteo erwähnt. Diese produzierte und verkaufte unter Umgehung der Lizenzabgaben AO-Produkte. Die Reaktion der AO blieb nicht aus, wie die Aussagen des damaligen Obmannes Robert Schneider belegen: «It could not be tolerated, he asserted, that a foreign group bearing the name AO collaborated with other companies, such as Osteo.» ${ }^{61}$ Folgerichtig wurde zur Verbesserung der Marktkontrolle am 25. November 1972 die AO International (AOI) als Dachorganisation aller nationalen AO-Organisationen gegründet. Deren erster Präsident, Hans Willenegger (1973-1983), widmete sich intensiv der systematischen weltweiten Schulung.

Eine neue Dynamik erhielt das Beziehungsgeflecht zwischen der AO und ihren Produzenten mit dem Markteintritt von Synthes USA. Diese Firma wurde 1974 von Straumann zusammen mit der Synthes AG Chur geschaffen, nachdem sich bis anhin das US-Geschäft nur schwach entwickelt hatte. Drei Jahre später übernahm der ursprünglich aus Bern stammende Ingenieur Hansjörg Wyss zwanzig Prozent der Aktien von Synthes USA. Er errichtete 1979 eine eigene Produktionsabteilung und reformierte die Verkaufsorganisation von Grund auf. Unter seiner Leitung steigerte die Firma zwischen 1977 und 1984 den Umsatz von 3,5 Mio. auf 28 Mio. und bis 1999 auf über 500 Mio. Dollar. ${ }^{62}$ Nachdem Synthes USA 1984 der Synthes AG Chur 2 Mio. CHF an Lizenzgebühren abgetreten hatte (Straumann: 4 Mio. CHF, Mathys: 5,6 Mio. CHF), überflügelte sie bereits zu Beginn der 1990er-Jahre die beiden Schweizer Betriebe. Die geänderte Struktur mit nunmehr drei Partnern gegenüber der Synthes AG Chur führte am 8. Dezember 1984 zur Gründung der AO-Stiftung mit einer neu geschaffenen Geschäftsleitung und dem Einsitz der Produzenten als stimmberechtigte Mitglieder. Im Zuge seines Markterfolges zahlte Wyss für den Betrag von insgesamt 54 Mio. USD alle Anteilhaber von Synthes USA sukzessive aus. Er besass nun denselben Status wie Mathys und Straumann. AO-Mitbegründer Maurice E. Müller sah durch die Schaffung der AO-Stiftung die ursprüngliche Idee seiner Institution zusehends durch kommerzielle Interessen in den Hintergrund gedrängt und stieg im Januar 1989 (zusammen mit Wyss) aus dem Stiftungsrat der AO-Stiftung aus. ${ }^{63}$

61 Schlich, Surgery, Science and Industry, S. 222 und Jeannet, Leading a Surgical Revolution, S. 223.

62 Breiding/Schwarz, Wirtschaftswunder Schweiz, S. 264.

63 Jeannet, Leading a Surgical Revolution, S. 167-168 und S. 254-255. 
Das enorme Wachstum von Synthes USA zeigt sich auch an folgenden Fakten: 1984 betrug der Marktanteil der Synthes AG Chur in Österreich 90 Prozent, in Deutschland 8o Prozent, in Belgien 70 Prozent, in Frankreich 50 Prozent, in Italien 10 Prozent. Die AO hatte zudem ihren Wirkungsbereich inzwischen von der Trauma- zur Hüft-, Rücken-, Gesichts- und selbst in die Veterinärchirurgie ausgeweitet. Keine zehn Jahre später war der amerikanische Betrieb wichtigster Partner der AO-Stiftung. ${ }^{64}$

Der Bedeutungszuwachs von Synthes USA im Laufe der 1980er-Jahre war jedoch erst der Auftakt. 1999 fusionierte das Unternehmen mit der 1989 gegründeten Stratec Medical und fasste dadurch in Europa und an der schweizerischen Börse unter dem Namen Synthes-Stratec Fuss. ${ }^{65}$ Im April 2004 übernahm das Unternehmen für 1,5 Mrd. CHF die Osteosynthese-Abteilung der Mathys Medizinaltechnik AG. Wie schon im Falle von Stratec Medical liess Hansjörg Wyss im Sinne der von ihm gewünschten Diversifikation die bestehenden (Zuliefer-)Betriebe unverändert. Der neu unter dem Namen Synthes, Inc. firmierende Konzern war von da an die dominante Medtech-Firma im Kanton Solothurn und alleiniger Partner der Synthes AG Chur. ${ }^{66}$

Die Synthes AG Chur nahm für den Erfolg der Produzenten weiterhin eine zentrale Rolle wahr. Die Bank Julius Bär brachte in einer Gesellschaftsstudie über Synthes-Stratec die Bedeutung der AO auf den Punkt: «Die AO betreibt primär Grundlagenforschung, deren Ergebnisse von den Partnerfirmen genutzt werden können. Daneben bietet sie Ausbildungskurse für Chirurgen und Operationssaal-Personal an mit Teilnehmerzahlen von jährlich über 1000o. So findet z.B. jedes Jahr im Dezember der Orthopädie-Kongress der AO in Davos statt. Ausbildungskurse und Kongresse stellen ein wichtiges Marketinginstrument für die Partnerfirmen dar, mittels welchem dem Fachpublikum der fachgerechte Einsatz der Produkte gelehrt wird sowie die Entwicklungen und Produkte gezeigt werden. ${ }^{67}$ Gemäss dem Aktienbroker Helvea hatte die AO zwischen 1960 und 2006215000 Chirurgen aus 122 Ländern und 110 ooo Operationsfachleute aus 168 Ländern geschult. ${ }^{68}$

Zwei Jahre nach der Übernahme der Mathys Medizinaltechnik AG einigten sich die Synthes Inc. und die AO-Stiftung auf ein neues Geschäftsmodell. Für 1 Mrd. CHF verkaufte die AO ihre ungefähr 3500 Patente an Synthes. Diese bezahlte der zur AO Technolgy umbenannten Synthes AG Chur anstelle der Lizenzgebühren nun einen jährlichen Fixbetrag für klar definierte Dienstleistungen wie Forschung und Schu-

\footnotetext{
64 Ebd., S.159-160 und S. 228.

65 Ebd., S. 256-257.

66 Ebd., S. 260-261.

67 Julius Bär (Hrsg.), Gesellschaftsstudie Synthes-Stratec, S.11.

68 Helvea, Synthes. Nailing Success, S.9.
} 
lung - eine Praxis, die auch nach der Übernahme durch J \& J fortgesetzt wurde. Die entsprechende Summe belief sich 2006 auf 50,7 Mio. CHF, zwischen 2012 und 2015 auf ungefähr 60 Mio. CHF. ${ }^{69}$ Auf dieser Basis setzt die AO-Stiftung mit heute ungefähr 320 Mitarbeitenden ihre Forschungs- und Schulungstätigkeit in verschiedenen klinisch spezialisierten Untergruppen fort. Bis heute bleibt die Technische Kommission der AO die einzige Instanz, die Synthes-Produkte genehmigen darf.7०

Nach der Jahrtausendwende ist die bereits gut positionierte Medizintechnik am Jurasüdfuss nochmals sprunghaft gewachsen. Neben dem durch die Synthes vorgenommenen Konzentrationsprozess traten mit der Übernahme der Osteo AG durch die Stryker AG (1996) und mit der Errichtung einer Zweigniederlassung in Solothurn durch die in Burgdorf ansässige Ypsomed (2004) zwei wichtige neue Unternehmen auf den Plan. Stryker verfügt heute in Selzach über 574 Vollzeitstellen, Ypsomed in Solothurn über 348 und in Grenchen bei seinem Décolletage-Hersteller Ypsotec nochmals über 87. Zwischen 2000 und 2004 sowie 2004 und 2008 hat sich die Bruttowertschöpfung ${ }^{71}$ der Medizintechnik im Kanton Solothurn (ohne Zulieferer und Dienstleister) jeweils verdoppelt. Letztere hat sich von der Euro- und Bankenkrise 2008 erholt und die Frankenstärke gut gemeistert. Im Jahre 2008 lag die kantonale Bruttowertschöpfung der Medtech bei rund 530 Mio CHF, was 10,72 Prozent des entsprechenden gesamtschweizerischen Medtech-Werts entsprach. ${ }^{72}$ Heute ist der Kanton Solothurn einer der vier bedeutendsten Medtech-Standorte der Schweiz, ${ }^{73}$ wie auch Roger Graber von der kantonalen Wirtschaftsförderung bestätigt: "Insbesondere in der Orthopädie wurde Wirtschaftsgeschichte geschrieben.» ${ }^{74}$ Die Führungsrolle in der Traumatologie und Orthopädie zeigt sich auch darin, dass 50 Prozent der in der Schweiz produzierten Implantate aus dem Kanton Solothurn stammen. ${ }^{75}$ Die Solothurner Medizintechnik ihrerseits konzentriert sich in hohem Mass auf die Region zwischen Solothurn und Grenchen. ${ }^{76}$

Siehe Anhang 1: Solothurner MedTech-Unternehmen, S.109

Jeannet unterstützt diese Einschätzung: «This is the region where the watch industry had, at one time, reigned supreme with large operations in several towns in

\footnotetext{
69 Jeannet, Leading a Surgical Revolution, S. 266-267 und 307.

70 Jeannet, Leading a Surgical Revolution, S. 319-322 und Helvea, Synthes. Nailing Success, S.11.

71 Die Bruttowertschöpfung ist der Gesamtwert der im Produktionsprozess erzeugten Waren und Dienstleistungen, abzüglich Vorleistungen, d. h. der von der Firma geschaffene Mehrwert, in: Graber/Müller, Life Science Landschaft Solothurn, Folie 16.

72 Ebd.

73 Biedermann, Peter: Ein Netzwerk am Puls der Medizintechnik, in: SO Economy 16.6.2011.

74 Graber, Vom Uhrenhersteller zum Schweizer Hotspot für Medizintechnik, in: Handelskammerjournal 7.6.2016.

75 Ebd.

76 Graber/Müller, Life Science Landschaft Solothurn, Folie 15.
} 
Solothurn. The medical cluster, led by the AO trauma inspired operations, having long eclipsed the watch industry in both importance and impact.» ${ }^{77}$ Neben dem vorhandenen Fachwissen in der Präzisionsmechanik ist die Region kleinräumig, die Wege sind kurz. Der ganze Berufsraum ist übersichtlich, reicht bis in die Uhrenindustrie zurück und verfügt deshalb über eine ausgeprägte Kontinuität, die nicht bloss die industrielle Produktion, sondern die ganze Lebenswelt umfasst. ${ }^{78}$ Sara Koch, Leiterin der Wirtschaftsförderung, folgert auf der Basis dieser Rahmenbedingungen: «Medizintechnik bietet sehr viel Potenzial für die Verbindung von Startups, Innovation und bestehenden Unternehmungen. . ${ }^{79}$ In ihrem historisch gewachsenen, traditionellen Umfeld ist die Medtech-Landschaft nach wie vor geprägt von KMU. So waren 2018 von den 92 Medtech-Firmen 36 Mikrounternehmen mit 1-9 Mitarbeitenden, 44 kleine Unternehmen mit 10-49 Mitarbeitenden, 8 mittlere Unternehmen mit 50-249 Mitarbeitenden und bloss 4 grosse Unternehmen mit mehr als 250 Mitarbeitenden. Die letztgenannten Firmen verfügten im selben Jahr allerdings über 3230 Arbeitsplätze und damit über klar mehr als die Hälfte der insgesamt 6200 Beschäftigten. ${ }^{80}$ Die vorwiegend klein- bis mittelbetriebliche Struktur ist zu einem guten Teil bedingt durch die insgesamt 44 Zulieferbetriebe, die ganz oder teilweise für die Medizintechnik arbeiten. Sie beschäftigen alles in allem 2400 Personen. Viele unter ihnen sind hoch spezialisierte Unternehmen und nehmen eine differenzierte Nachfrage nach kleinen Serien wahr. ${ }^{81}$ Dazu gehören Betriebe wie die Hakama in Bättwil, die Gehäuse aus Feinblech produziert, der Hersteller von hoch entwickelten Präzisionswerkzeugen Fraisa in Bellach ${ }^{82}$ oder die in Derendingen ansässige Sphinx, die Bohrer mit einem Durchmesser von 0,03 mm produziert. ${ }^{83}$

Obschon der Kanton Solothurn im internationalen Vergleich unbestritten eine Hochlohnregion ist, konnte sich die Medizintechnikbranche am Jurasüdfuss bisher halten. Wichtige Gründe für diesen Sachverhalt sind der hohe Grad an Automatisierung, die Fachkompetenzen der Arbeitskräfte sowie der Genius von Ingenieuren und Managern wie Mathys, Straumann oder Wyss. ${ }^{84}$ Dank dieser Voraussetzungen ist die Produktivität bis heute unerreicht, wie Jeannet als einer unter vielen fest-

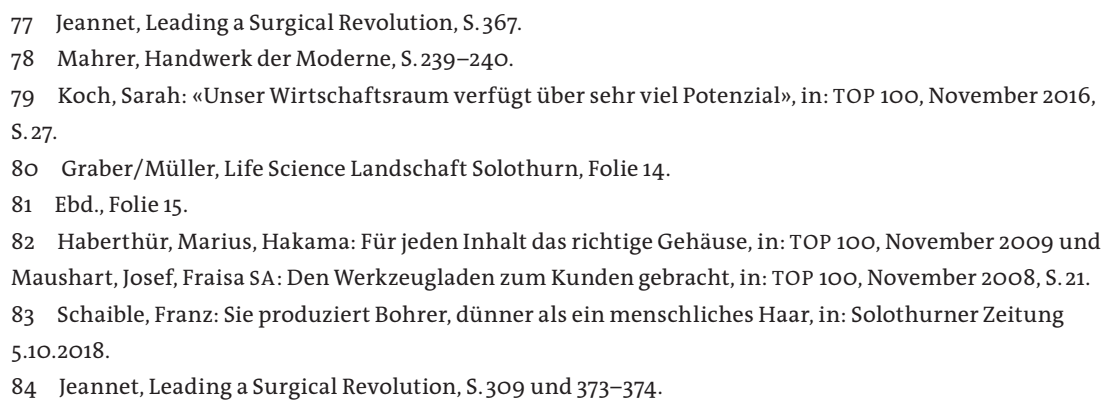


hält: «The Swiss operation was kept at such high productivity level that the plants became the model factories in the global DRS Trauma segment. ${ }^{85}$ Entsprechend weisen die Produkte eine überdurchschnittlich hohe Bruttowertschöpfung auf. Pro Arbeitnehmer und Jahr beträgt diese 150000 CHF. ${ }^{86}$ Wie bedeutend diese Tatsachen für die gesamte Industriestruktur der Region sind, unterstreicht Andreas Brand, ehemaliger Chef des Amtes für Berufsbildung, Mittelschulen und Hochschulen des Kantons Solothurn: «Ich denke, durch die Entwicklung von wertschöpfungsintensiven Betrieben, vor allem in der Medizintechnik, wurde die industrielle Basis in letzter Zeit gestärkt.» ${ }^{87}$ So weit einige Andeutungen, die die Konkurrenzfähigkeit der Medizintechnik am Jurasüdfuss zu erklären vermögen. Mit den Erfolgsfaktoren im Einzelnen befassen wir uns in Kapitel 6.

Die Entwicklung der Medizintechnik am Jurasüdfuss lässt sich seit dem Startpunkt 1958 auch quantitativ aufzeigen. Die Angaben sind allerdings bis zu Beginn des 21. Jahrhunderts mit Vorsicht zu geniessen. Bei den periodischen Betriebszählungen des Eidgenössischen Statistischen Amtes (heute: Bundesamt für Statistik) ändern sich die Kriterien zwischen 1955 und 1995 regelmässig. Ab 1995 verfügen wir über die Zahlen von NOGA. Für unsern Untersuchungsbereich betreffen diese die folgenden Codes:

266000 Herstellung von Bestrahlungs- und Elektrotherapiegeräten und elektromedizinischen Geräten

325001 Herstellung von medizinischen und zahntechnischen Apparaten und Materialien

325002 Herstellung von orthopädischen und prothetischen Erzeugnissen 325003 Zahntechnische Laboratorien

Als nützliche Quellen dienen ab 2007 die jährlichen Darstellungen der Beschäftigtenzahlen in den hundert wichtigsten Betrieben des Kantons Solothurn. ${ }^{88}$ Ab 2016 kommt auf kantonaler Ebene die Umfrage der Wirtschaftsförderung des Kantons Solothurn hinzu, die namentlich auch die Zulieferbetriebe berücksichtigt und dadurch die genausten Zahlen liefert. Wie stark diese variieren, mag folgendes Beispiel für 2016 aufzeigen: Über NOGA wurden für dieses Jahr 2582 Beschäftigte ausgewiesen. In der erwähnten Umfrage zu den hundert Betrieben mit den meisten Beschäftigten wurden allein für die fünf grössten Betriebe 3272 Vollzeitbeschäftigte

85 Ebd., S.310.

86 Graber/Müller, Life Science Landschaft Solothurn, Folie 16.

87 Brand, Andreas, zit. nach: Hafner, Die Krise und ihre Bewältigung, S.40.

88 Bundesamt für Statistik, NOGA 1995-2008 und 2011-2016 und TOP 100, November 2007-November 2018. 
angegeben. Die Wirtschaftsförderung erwähnte - ebenfalls für 2016 - 5860 Vollzeitbeschäftigte für die Medizintechnik des Kantons Solothurn. ${ }^{89}$

Vorbehältlich dieser Einschränkungen lassen sich die Wachstumstendenzen nachzeichnen. Die Eidgenössische Betriebszählung 1955 gibt für die «Berufsgruppe 36: Feinmechanik, medizinische Instrumente und Apparate» 43 Betriebe mit insgesamt 395 Beschäftigten an, davon 371 Männer. Die «Untergruppe medizinische Instrumente und Apparate» bringt es in dieser Statistik auf genau vier (!) Personen. Unsere Feststellung, wonach die Medizintechnik im Kanton Solothurn vor April 1958 nicht existent war, wird dadurch bestätigt. ${ }^{\circ \circ}$ Gegenüber 1955 ist die «Berufsgruppe 3507: Feinmechanische, optische, medizinische Apparate, Instrumente» 1965 auf insgesamt 22 Betriebe mit 243 Beschäftigten zurückgegangen. Ein Vergleich ist indessen nur sehr beschränkt möglich, weil die medizinischen Instrumente und Apparate nicht mehr gesondert aufgeführt sind. ${ }^{91}$ Wiederum zehn Jahre später, bei der Eidgenössischen Betriebszählung 1975, wird die "Kategorie 354" nur noch mit «Feinmechanik/Optik» angegeben. Die Zahl der Beschäftigten dieser Gruppe ist im Kanton Solothurn auf 534 Personen angestiegen. Erneut werden medizinische Instrumente und Apparate nicht spezifisch erwähnt, doch weist das Wachstum im Bereich Feinmechanik/Optik indirekt auf steigende Beschäftigtenzahlen in der Medizintechnik hin, was vor allem mit der Entwicklung der Mathys Medizinaltechnik AG zusammenhängt. Betrug der Frauenanteil 1965 noch 10,28 Prozent, kletterte er zehn Jahre später auf 18,7 Prozent - ein möglicher Hinweis auf verstärkte Arbeitsteilung und Zunahme der Beschäftigten in grösseren Betrieben. ${ }^{92} 1985$ weist die «Kategorie 3514: Maschinen/Präzisionswerkzeug» für den Kanton Solothurn $1352 \mathrm{Be}$ schäftigte in siebzig Betrieben auf. ${ }^{93}$ Dass diese Branche nach wie vor ausgesprochen KMU-lastig war, zeigt eine ergänzende Statistik des Finanzdepartementes des Kantons Solothurn. Gerade einmal sieben Betriebe beschäftigten mehr als fünfzig Arbeitnehmer. Diese teilten sich wie folgt auf:

Kategorie 50-99 Beschäftigte: drei Betriebe mit total 200 Mitarbeitern Kategorie 100-199 Beschäftigte: drei Betriebe mit total 417 Mitarbeitern Kategorie 200-499 Beschäftigte: ein Betrieb mit 202 Mitarbeitern $^{94}$

\footnotetext{
89 Bundesamt für Statistik, NOGA 2016, TOP 100, November 2016, S. 5, 7 und 13 und Graber, MedTech-Landschaft Kanton Solothurn, S.4.

90 Eidg. Statistisches Amt, Eidg. Betriebszählung 1955, S.40-41 und 236-237.

91 Eidg. Statistisches Amt, Eidg. Betriebszählung 1965, S. 212-213.

92 Eidg. Statistisches Amt, Eidg. Betriebszählung 1975, S. 398-399.

93 Bundesamt für Statistik, Eidg. Betriebszählung 1985, S. 152-153.

94 Finanzdepartement des Kantons Solothurn, Betriebszählung Kanton Solothurn 1985, S. 26-27.
} 
Wiederum eine neue Kategorie findet sich in der Betriebszählung 1995 unter «33: Humanmedizinische und Präzisionsinstrumente». Der Kanton Solothurn zählt 1995 für diese Berufsgruppe 145 Betriebe mit insgesamt 5004 Beschäftigten. Auch wenn sich diese Zahl mit den vorangegangenen Jahrzehnten aufgrund der geänderten Kategorienbildung kaum vergleichen lässt, kommt in ihr indirekt das Wachstum der Medizintechnik zum Ausdruck (Mathys Medizinaltechnik AG, Stratec Medical usw.) $)^{95}$

Seit 1995 hat das Bundesamt für Statistik auf die NOGA-Klassifizierung umgestellt. Die entsprechenden Zahlenreihen sind aus den erwähnten Gründen unpräzis (vor allem keine Zahlen aus Zulieferbetrieben). Trotzdem bilden sie tendenziell das rasante Wachstum der Medizintechnik ab. So fand zwischen 1995 und 2008 mit einer Beschäftigungszunahme von 952 auf 1893 nahezu eine Verdoppelung statt. Von 2008 bis 2016 ist eine stetige Zunahme auf 2528 Beschäftigte festzustellen.

Die aufgeführten Zahlen aus den Betriebszählungen 1955-1995 und NOGA ab 1995 sind für die Medizintechnik im Kanton Solothurn weder besonders genau noch über den Zeitraum hinweg vergleichbar. Trotzdem lassen sich aus ihnen Entwicklungstendenzen ablesen. Genauere Angaben finden sich bei den Beschäftigtenzahlen der vier ausgewählten Betriebe.

Siehe Anhang 2: Beschäftigte in der Medizintechnik im Kanton Solothurn 19952016, S. 110

\subsection{Mathys steht am Ursprung}

Zwischen 1958 und der Jahrtausendwende ist die Firmengeschichte praktisch deckungsgleich mit der Geschichte der Medizintechnik am Jurasüdfuss: «Mathys AG bildete den Grundstein für die Wachstumsbranche der Medizintechnik am Jurasüdfuss. ${ }^{96}$ Wichtige Daten und Fakten zu den Anfängen und der Weiterentwicklung der Mathys AG finden sich deshalb bereits in Kapitel 5. Seit 1958 hat die Firma ihren Namen mehrmals gewechselt. Von Robert Mathys über Robert Mathys AG, Mathys AG, Mathys Medizinaltechnik AG bis zu Mathys AG Bettlach (ab 2003). Der Einfachheit halber benutzen wir nachstehend als Firmennamen die Bezeichnung «Mathys».

Unmittelbar nach der Begegnung mit dem Chirurgen Maurice E.Müller am 8. April 1958 (siehe S. 25) war Robert Mathys sen. von den Möglichkeiten der Medi-

95 Bundesamt für Statistik, Eidg. Betriebszählung 1995, S. 64-65.

96 Scheidegger, Solothurner Pioniergeist, S. 64. 
zintechnik begeistert. Der Einsatz von Mathys war umso erstaunlicher, als er Müller versprechen musste, mit der serienmässigen Fabrikation zuzuwarten und die Produkte während dreier Jahre ausschliesslich der AO zu verkaufen. Damit wollten die Chirurgen sichergehen, dass das Instrumentarium nur von Ärzten angewendet wurde, die die AO-Kurse besucht hatten und auf diese Weise eine einwandfreie Handhabung gewährleisteten. Müller wollte folglich das Instrumentarium (Platten, Schrauben, Nägel) technologisch zur Reife bringen, danach die Anwender schulen und erst in einem nächsten Schritt mit den Produkten an den Markt gehen. Dies umso mehr, als die erforderliche Technologie noch in den Kinderschuhen steckte. «Das Know-how dieser Fertigung war noch nicht Allgemeingut; d.h. die Schneidemittel, Drehbänke und andere Hilfsmittel benötigten bestimmte Anforderungen für die Verarbeitung der rostfreien Materialien, weil diese relativ ‘zähfest` waren.» ${ }^{97}$

Andere von Müller kontaktierte Hersteller hatten diese Geduld nicht, auch wenn ihnen der Chirurg innert spätestens vier Jahren Millionenumsätze versprach. Mathys hingegen stieg auf den mit Handschlag besiegelten Handel ein. Beinahe schon obsessiv verfolgte er das Projekt, sodass seine übrige Produktion ins Hintertreffen geriet. Seine Mittel brauchte er vollumfänglich für die Entwicklung der neuen Instrumente und Bestandteile, sodass er 1960, immer noch vor Beginn der Serienproduktion, 300000 CHF Schulden hatte-damals eine beträchtliche Summe. ${ }^{98}$ Robert Mathys jun. übertreibt keineswegs, wenn er festhält: «Das Engagement hat meinem Vater fast den Kopf gekostet.»99 Maurice E. Müller beteuerte deshalb den ortsansässigen Banken, dass Mathys bald «loslegen» und die Schulden zurückzahlen würde.

Nur dank seiner Fokussierung war es Robert Mathys sen. möglich, bis zum ersten AO-Seminar im Dezember 1960 zwanzig Sets mit je sechs Aluminiumboxen zu liefern und so das "Durcheinander in den Operationssälen" zu ordnen. ${ }^{100}$ Bei der Beurteilung seiner Pionierleistung ist ferner zu berücksichtigen, dass die Rahmenbedingungen in der Region alles andere als einfach waren, wie Robert Mathys jun. betont: «Die Uhrenindustrie kontrollierte auf dem Platz Grenchen alles, was Fachkräfte betraf. Die Décolleteure konnten nirgendwo frei arbeiten. Qualifizierte Arbeitskräfte durften nicht in andere Branchen abwandern. Décolleteure durften also nicht einfach zu Mathys gehen. Mein Vater musste diese anlernen.»101

Mathys' Geduld sollte sich lohnen. Seine Firma wurde der beherrschende Medizintechnikbetrieb am Jurasüdfuss und blieb dies bis zum Ende des Jahrhunderts. Der Erfolg lässt sich auch in Zahlen ausdrücken. Im Jahre 1997 hatte die Mathys

97 Gespräch mit Robert Mathys jun.

98 Heim, Das Phänomen AO, S. 58 und Schlich, Surgery, Science and Industry, S. 55.

99 Gespräch mit Robert Mathys jun.

100 Jeannet, Leading a Surgical Revolution, S. 80.

101 Gespräch mit Robert Mathys jun. 


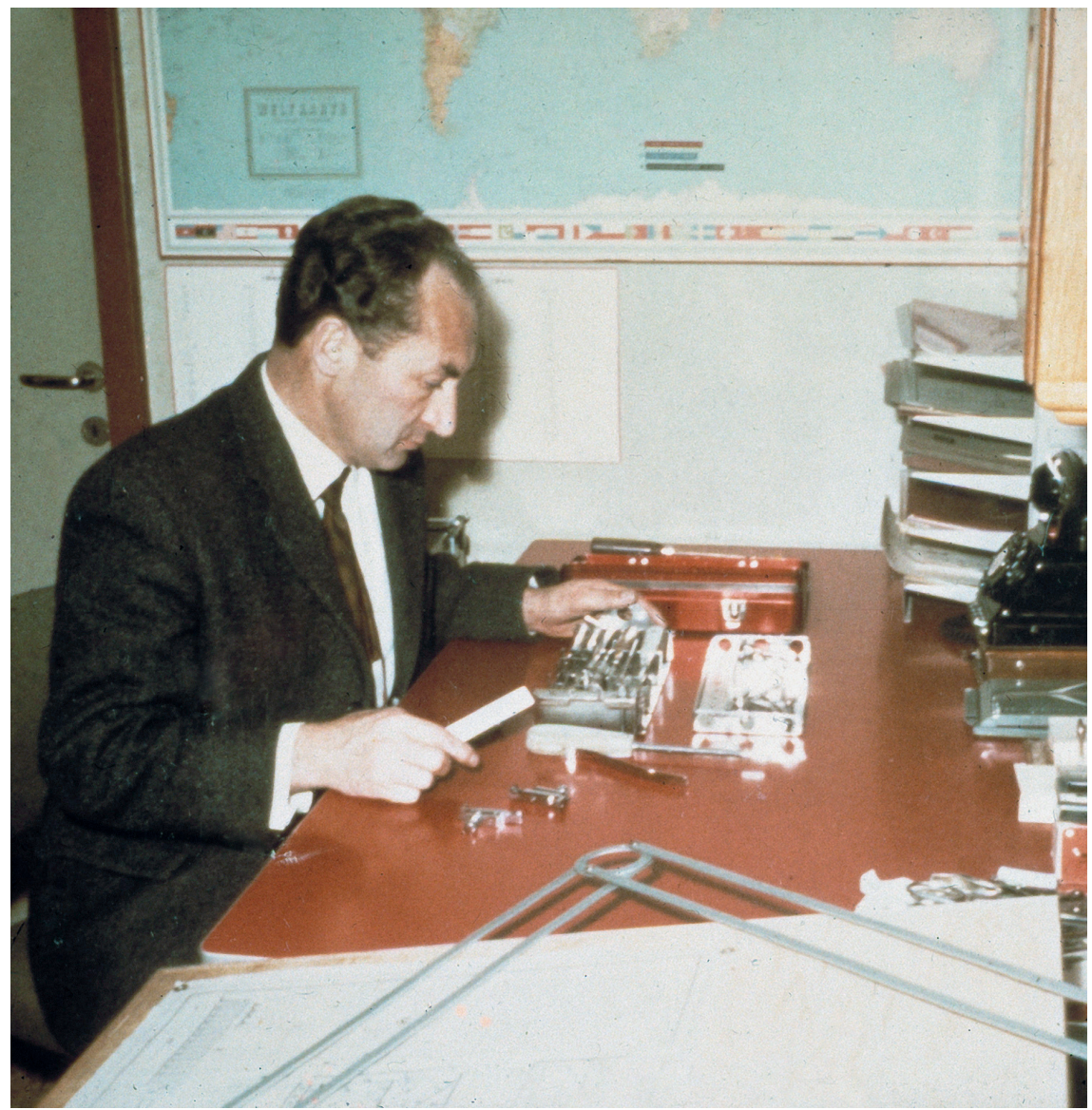

Robert Mathys sen. bei der Arbeit

(c) MATHYS AG, Bettlach 


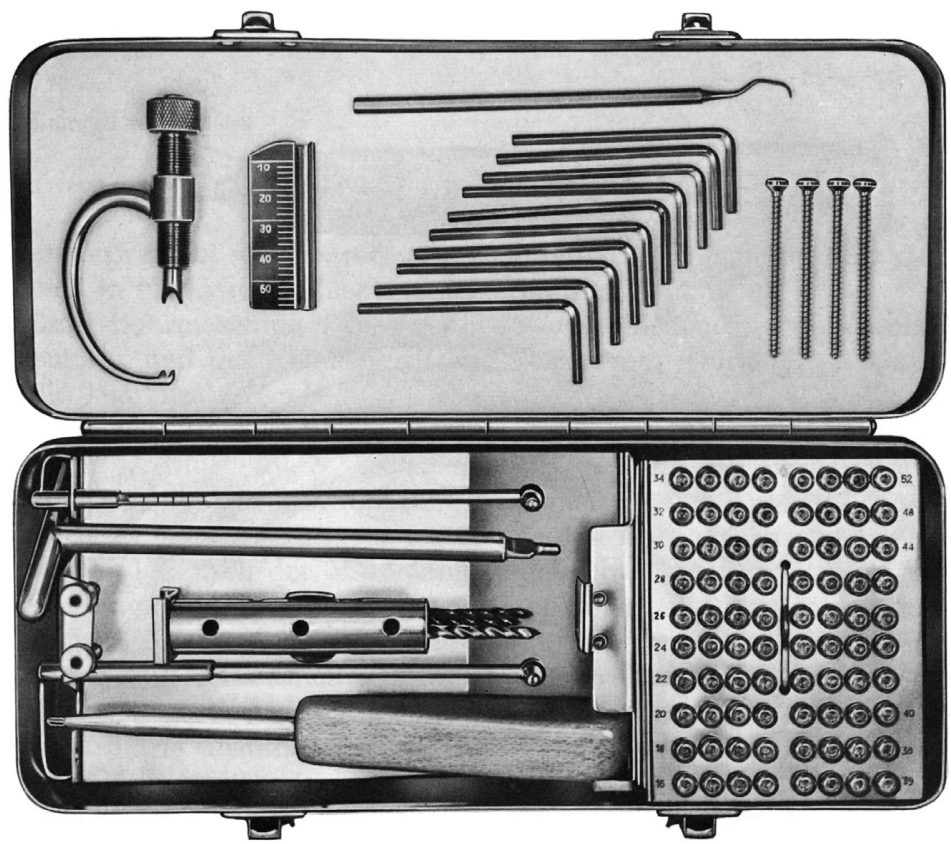

Set mit Schrauben und Werkzeugen

Copyright by AO-Foundation, Switzerland
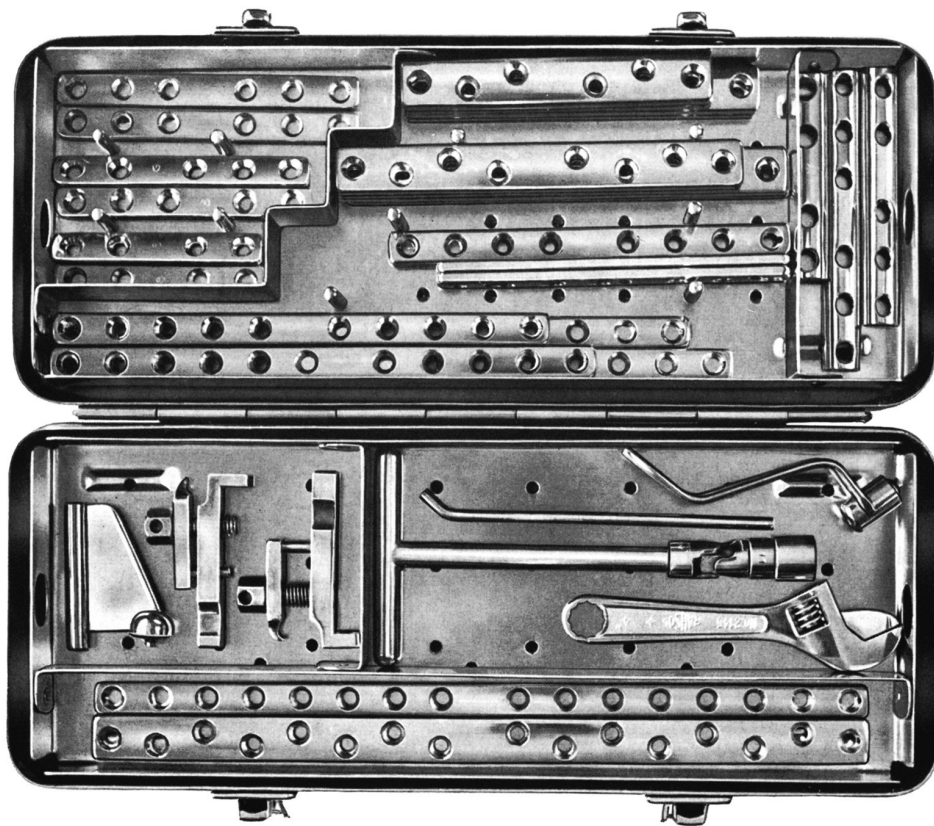

Set mit Druckplatten und Instrumenten

Copyright by AO-Foundation, Switzerland 
Medizinaltechnik AG 16 internationale Zweigniederlassungen und über fünfzig Verkaufsstellen weltweit. Insgesamt zählte sie zu diesem Zeitpunkt 1100 Angestellte, davon 600 im Kanton Solothurn und 500 im Ausland. ${ }^{102}$

Wie erwähnt, begann 1960 infolge von Materialschäden die Zusammenarbeit mit dem Reinhard-Straumann-Forschungsinstitut in Waldenburg (siehe S.26-27). Mathys bestellte nun den Stahl bei Straumann, zumal das Instrumentarium für die AO identisch sein musste. Später wurde Straumann auch zum Lieferanten des von ihm entwickelten Titans. Die Leute aus Waldenburg wiederum verfügten zu Beginn nicht über die Infrastruktur für die Produktion der Schrauben, Implantate und Nägel, sodass Mathys in die Bresche sprang. Trotz dieser partiellen Kooperation standen die beiden Betriebe in einem ungeklärten Konkurrenzverhältnis. ${ }^{103}$ Mathys hatte zunächst den Eindruck, dass die "Basler Fraktion» mit Willenegger und Allgöwer die Waldenburger Firma bevorzugen und ihn ausbremsen würde. Schliesslich trafen sich die beiden Kontrahenten 1962 oder 1963 - der Termin ist nicht mehr genau bekannt - auf mittlerem Weg zwischen Bettlach und Waldenburg, im Bahnhofbuffet Olten. Dort teilten sie per Losentscheid den (noch zu bearbeitenden) Weltmarkt untereinander auf. Ihr Vorgehen mag illustrieren, wie stark für die beiden Fachleute ihre Mission und die entwickelten Hilfsmittel im Vordergrund standen und welch «notwendiges Übel» demgegenüber die Marktaufteilung war. Mitarbeiter von Straumann hatten Zettel mit Ländern und Kontinenten vorbereitet. Als Älterer zog Mathys jeweils zuerst. Schliesslich erhielt er unter anderem Afrika, Asien, den Nahen Osten, Frankreich, Italien und Österreich, während Straumann die Schweiz, Grossbritannien, Skandinavien, Lateinamerika und Nordamerika zu seinem Absatzgebiet zählte. Deutschland wurde als potenziell wichtigster Markt in einen südlichen und nördlichen/nordöstlichen Teil (inkl. DDR) aufgeteilt, nachdem Robert Mathys am nahen Bahnhofskiosk eine Deutschlandkarte besorgt hatte ... ${ }^{104}$

Geregelte Marktbeziehungen unter den beiden Produzenten waren die Voraussetzung dafür, dass die AO den Verkauf ihres neu entwickelten Instrumentariums überhaupt freigeben konnte. Dass die Synthes AG Chur mit Mathys und Straumann erst am 21. November 1963 einen Vertrag abschliessen konnte, ist deshalb kein Zufall. Die Interessenkonflikte zwischen den beiden Firmen mussten zuerst behoben werden, und mit dem Vertrag erkannten beide den Primat der Chirurgen an, da sie an Glaubwürdigkeit und langfristigem Erfolg interessiert waren. ${ }^{105}$

Aufbau und Expansion der Firma Mathys lassen sich teilweise mit der Persönlichkeit von Robert Mathys sen. erklären. Neben seinem Einsatz und seiner Begeis-

102 Schlich, Surgery, Science and Industry, S.164.

103 Jeannet, Leading a Surgical Revolution, S.121-123 und S.151-153.

104 Aiolfi, Sergio: Müller und Motörli Röbu, in: NZZ Folio, November 2005.

105 Ebd., Heim, Das Phänomen AO, S. 93-94 und Schlich, Surgery, Science and Industry, S. 60. 


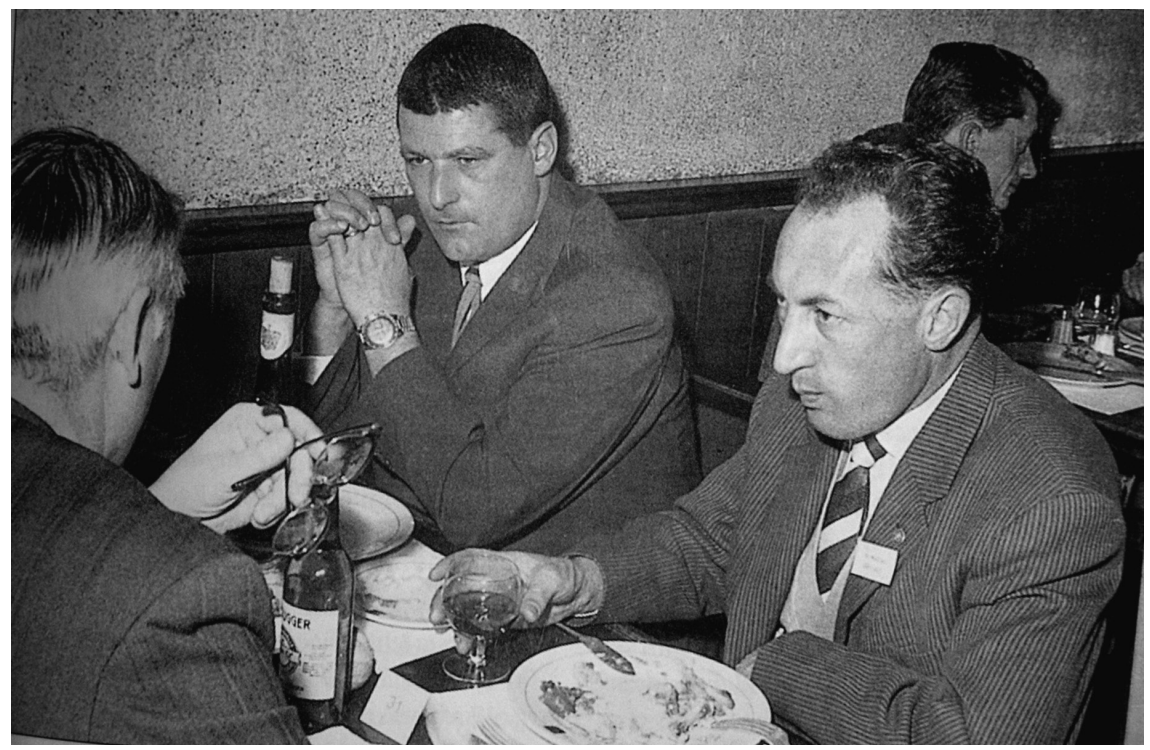

Robert Mathys sen. und Fritz Straumann nicht im Bahnhofbuffet Olten, sondern bei einem Mittagessen während eines AO-Kurses

Copyright by AO-Foundation, Switzerland

terungsfähigkeit zeichneten ihn eine intuitive Erfassung chirurgischer Problemstellungen, Wissensdurst, Beharrlichkeit ebenso wie ein fundiertes Praxiswissen aus. ${ }^{106}$ Anton Cotting, zwischen 1977 und 2005 leitend in Konstruktion und Entwicklung der Firma Mathys, charakterisiert ihn als «sehr innovativ» und «unglaublich kreativ». Und weiter: «Mit seinen Skizzen, verfasst auf irgendwelchem Papier oder notfalls auf einem Stofftischtuch, überhäufte er uns mit seinen 'Visionen`, die wir dann möglichst kurzfristig in funktions- und fabrikationsgerechte Produkte umzusetzen versuchten. ${ }^{107}$ Neben technischen standen für Robert Mathys sen. auch ethische Fragen im Vordergrund, wie eine Interviewantwort in der Solothurner Zeitung zu seinem 75. Geburtstag zeigt: «Es war für mich klar, dass meine Zukunft in der Mithilfe zur Pflege von Hilfebedürftigen und nicht in der Zerstörung liegen müsste. Aus diesem Grunde lehnte ich auch damals einen Grossauftrag einer Waffenfabrik ab.»108 Im selben Beitrag schimmert gleichzeitig durch, dass er kei-

106 Heim, Das Phänomen AO, S. 49.

107 Cotting, Anton: Ausgewählte Beispiele gemeinsamer Entwicklungsarbeiten, in: RMS Foundation (Hrsg.), Engagement in zweiter Generation, S. 22.

108 Jäggi, Daniela: Robert Mathys arbeitet nach der Devise: Marktlücken erkennen und diese schliessen, bevor es andere tun, Interview in: Solothurner Zeitung 31.1.1996. 


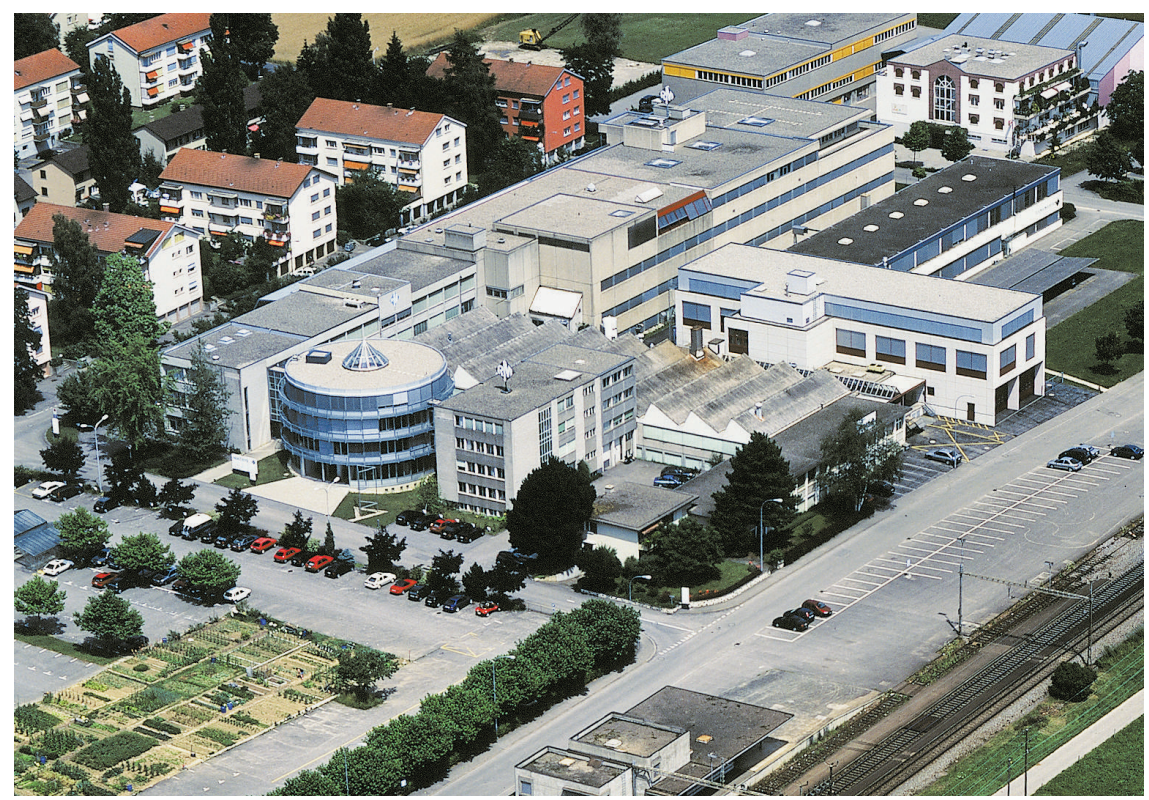

Das Firmenareal um die Jahrtausendwende. Der schrittweise Ausbau erfolgte nach der Devise: «Immer so viel wie nötig»

(c) MATHYS AG, Bettlach

neswegs bloss weltfremder Idealist war. Seine Devise sei «Lücken zu erkennen und diese zu schliessen, bevor es andere tun". ${ }^{109}$ Mathys selbst und seine Familie lebten bescheiden, die Reinvestitionen erfolgten jeweils über Eigenmittel. Typisch für sein unkompliziertes Engagement waren Flüge quer durch Afrika, die der passionierte Flieger selber unternahm, um seine Produkte kostenlos in Spitälern zu demonstrieren. ${ }^{110}$ Ohne Zweifel trugen diese Eigenschaften zur ausserordentlich engen Zusammenarbeit mit Maurice E. Müller bei. Thomas Schlich bezeichnet diese als Symbiose ("a unique collaboration between surgery and industry»). ${ }^{111}$

Nach dem Vertrag der Synthes AG Chur mit den beiden Produzenten Mathys und Straumann wuchs die Bettlacher Firma sprunghaft. Schon bald wurde klar, dass man nicht alles selber produzieren konnte. Mathys AG suchte die Zusammenarbeit mit anderen auf Décolletage spezialisierten Betrieben wie etwa Rieger in Grenchen. Später beteiligte sich Mathys an den Firmengründungen von Safrina

109 Ebd.

110 Jeannet, Leading a Surgical Revolution, S. 202 und 376.

111 Schlich, Surgery, Science and Industry, S. 55. 
Safnern (1972), der Almaco AG in Oensingen/Balsthal (1978), erweiterte die eigene Produktion in Grenchen (1996) und lagerte die Logistik nach Selzach aus (1998). Nach der Übernahme durch Synthes 2004 wurde die Almaco vom neuen Besitzer, der auch die Zweigstellen Grenchen und Selzach weiterführte, übernommen. Nach Aussagen von Robert Mathys jun. stammte stets ungefähr ein Drittel der Produktion von Zulieferern. ${ }^{112}$

1967 vergrösserte die Mathys AG ihr Spektrum von der Unfallchirurgie zur Orthopädie. Sie produzierte für die von Maurice E. Müller gegründete Firma Protek Implantate. Anton Cotting konkretisiert diese Phase im Rückblick: «Die Aufgaben wurden immer umfangreicher, zumal Produktideen aus unterschiedlichen Anwendungsgebieten und auch für verschiedene Partner zu entwickeln waren. Dazu gehörten Implantate und Instrumente für die Osteosynthese im Rahmen der Zusammenarbeit mit der AO-Gruppe (Arbeitsgemeinschaft für Osteosynthese-Gruppe), für den Gelenkersatz der Produktelinien der Firmen Mathys, Protek und Allopro sowie den Dentalbereich von Mathys.»113 Nachdem 1997 Protek an Sulzer verkauft worden war, produzierte Mathys unter eigenem Namen weiterhin orthopädische Produkte, die Gelenkersatz für Knie, Hüfte, Schultern sowie Knochenersatzmaterial umfassten. Die Orthopädie wurde nach dem Verkauf der Traumatologie an Synthes als Mathys Orthopedics AG mit stark steigenden Wachstumszahlen weitergeführt. ${ }^{114}$ Die Expansion spiegelt sich in den Erweiterungen 1963, 1966, 1968/69 sowie in der Entwicklung des Umsatzes wider. Dieser stieg von 2 Mio. CHF im Jahre 1966 auf 98 Mio. CHF 1985. ${ }^{115}$ Zu Beginn der 1980er-Jahre hatte Mathys 322 Beschäftigte in der Schweiz und 89 im Ausland. Mittlerweile belieferte die Firma weltweit 8000 Spitäler mit insgesamt 3000 Artikeln, zwei Drittel davon Implantate und ein Drittel Instrumente. Der Exportanteil betrug 95 Prozent. ${ }^{116}$ Bis 1997 verfügte Mathys über 16 internationale Zweigniederlassungen und auf dem ganzen Globus verteilt über mehr als fünfzig Verkaufsstellen. Die Beschäftigtenzahl war inzwischen auf 1100 Angestellte angewachsen, davon 600 in der Schweiz. Die internationale Ausdehnung der Produktion war nicht zuletzt auf Importzölle zurückzuführen. So entstanden etwa Produktionsstätten in Delhi und sogar in Moskau (1993). Grosse Erfolge hatte Mathys unter anderem auch in der DDR, wo das zentralisierte Gesundheitssystem eine strikte Kontrolle über die sachgemässe Anwendung des Instru-

\footnotetext{
112 Gespräch mit Robert Mathys jun.

113 Cotting, Anton: Ausgewählte Beispiele gemeinsamer Entwicklungsarbeiten, in: RMS Foundation (Hrsg.), Engagement in zweiter Generation, S. 22.

114 Jeannet, Leading a Surgical Revolution, S. 371.

115 Ebd., S.146.

116 Schlich, Surgery, Science and Industry, S. 156 und kt: Instrumentenwelt der Chirurgie, in: Solothurner Zeitung 6.7.1981.
} 


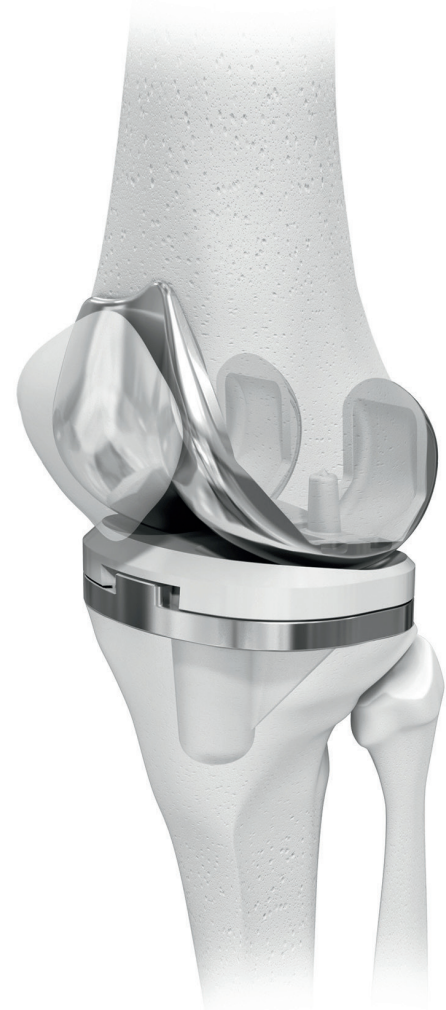

Kniesystem in der Gesamtschau (c) MATHYS AG, Bettlach
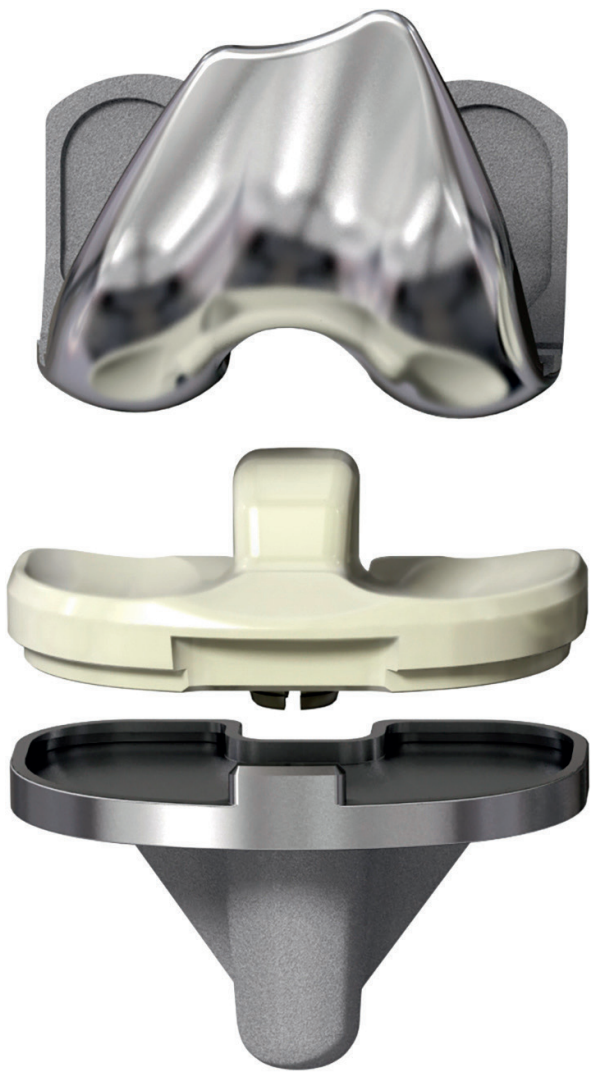

Knieprothese um 1995

(C) MATHYS AG, Bettlach 
mentariums gewährleistete. Nach einem kurzen Unterbruch, bedingt durch westdeutsche, an den obligatorischen Bezug von BRD-Produkten gekoppelte Kredite, hatte Mathys in der DDR ein Quasi-Monopol. ${ }^{117}$

Dank ihres stetigen Wachstums hatte Mathys AG genügend Polster, um 1998 die Asien-Krise sowie die durch die Internetblase verursachte Rezession 2002 zu überstehen. ${ }^{118}$ Wie wichtig, gerade in wirtschaftlich schwierigen Zeiten, die stabilisierende Wirkung von Mathys für die gesamte Region war, illustriert eine Aussage des ehemaligen Gemeindepräsidenten Hans Kübli: «Glücklicherweise begann nach der Krise der Uhrenindustrie mit dem Verlust vieler Arbeitsplätze ... unter Führung der Firma Mathys in Bettlach der Aufschwung der Medizinaltechnik.»119

Neben dem wirtschaftlichen Erfolg hat Robert Mathys für die moderne Chirurgie Pionierarbeit geleistet. Als Beispiel unter vielen dient folgender Ausschnitt aus einer firmeninternen Broschüre: «Bereits Ende der 6oer-Jahre entwarf Robert Mathys ein Konzept, das die unphysiologische Reaktion der zementierten Hüftgelenkprothese in Knochen vermeiden sollte. Die erste unzementierte Prothese entstand: RM Isoelastic, eine Verbundkonstruktion von Metall und Polyacetalharz. Heute weisen praktisch alle neuen Protheseentwicklungen in die von Robert Mathys bereits vor zwanzig Jahren aufgezeigte Richtung ... $\nu^{120}$ Dazu kamen Userworkshops und Symposien, die Mathys zusätzlich zum AO-Angebot für Chirurgen und OPPersonal aus der ganzen Welt durchführte. ${ }^{121}$

Zur Intensivierung der Forschungstätigkeit gründete Robert Mathys 1985 die Robert Mathys Stiftung (RMS Foundation). 1993 wurde diese rechtlich und finanziell unabhängig von der Mathys AG. Neben ihrer Forschungstätigkeit übernahm die RMS Foundation auch Dienstleistungsaufträge für andere Firmen, insbesondere in Form von Werkstoffuntersuchungen, biomechanischen Prüfungen, Materialtests und Technologieevaluationen. Einer der Grosskunden ist heute beispielsweise De Puy Synthes. Die RMS Foundation zählte 1992 17, 200326 und 201736 Mitarbeitende. ${ }^{122}$

Nach der Fusion von Synthes USA und Stratec-Medical 1999 wuchs der Druck auf Mathys AG. Noch wehrte sich die Bettlacher Firma gegen eine Übernahme durch den nunmehr um einiges grösseren Konkurrenten. Trotzdem erfolgte im April

\footnotetext{
117 Schlich, Surgery, Science and Industry, S. 164, 167 und 175-176.

118 Schenker, Urs: Robert Mathys - Führung der Medizinaltechnik AG, in: RMS Foundation, Engagement in zweiter Generation, S.14.

119 Kübli, Hans: Robert Mathys - seine Verdienste für die Gemeinde Bettlach, in: RMS Foundation (Hrsg.),

Engagement in zweiter Generation, S. 52.

120 Mathys AG Bettlach, Ganzheitliche Medizinaltechnik, S. 6.

121 Ebd., S.12.

122 Mathys, Robert: Schlossern für die Gesundheit, in: Betschon/Betschon/Lindecker/Schlachter, Ingenieure bauen die Schweiz, S. 432 und Gespräch mit Robert Mathys jun., Zahlen ebenfalls von Robert Mathys jun.
} 


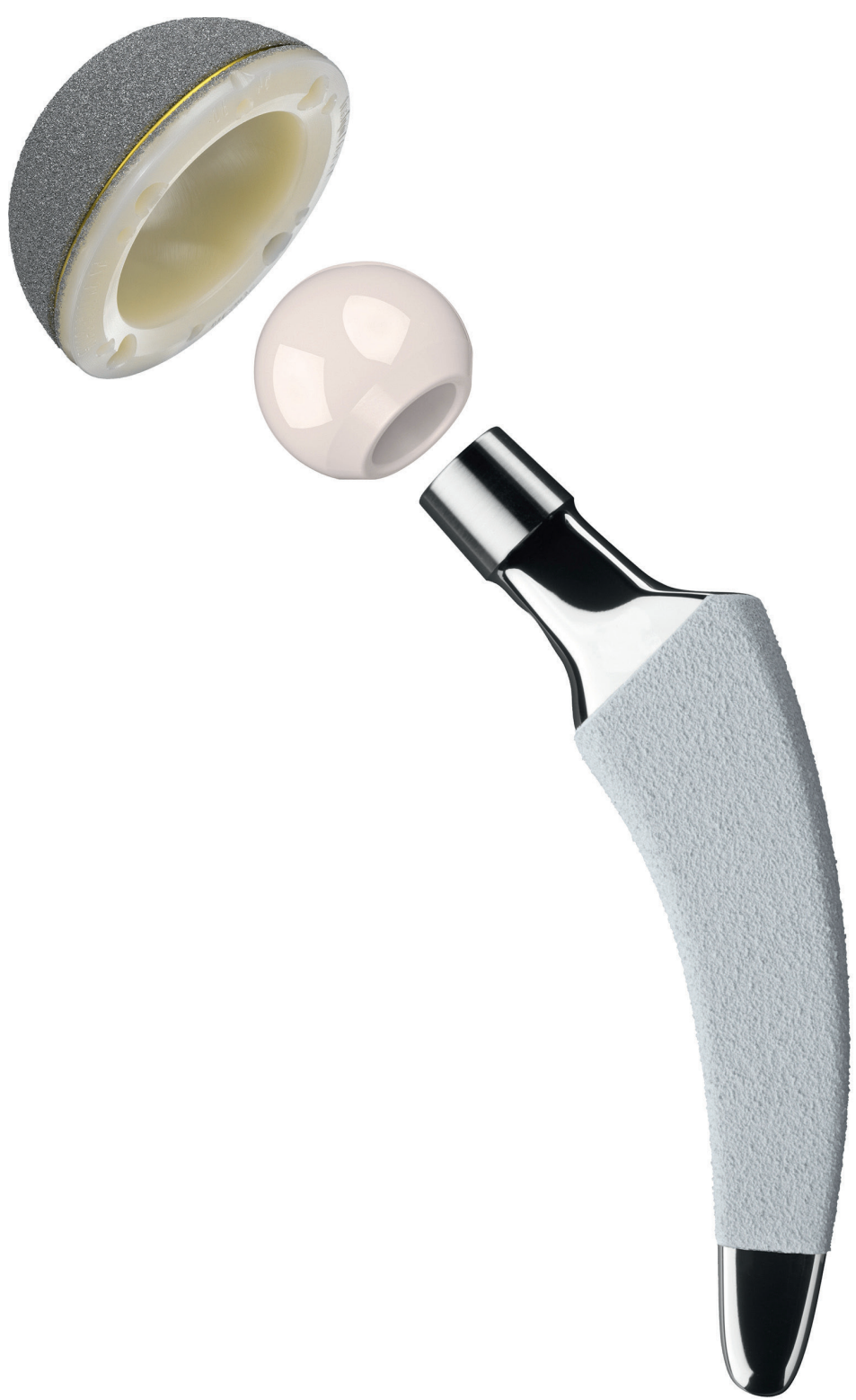

Der Hüftschaft mit beschichteter RMS-Pfanne erlaubt eine zementlose Fixierung (c) MATHYS AG, Bettlach 


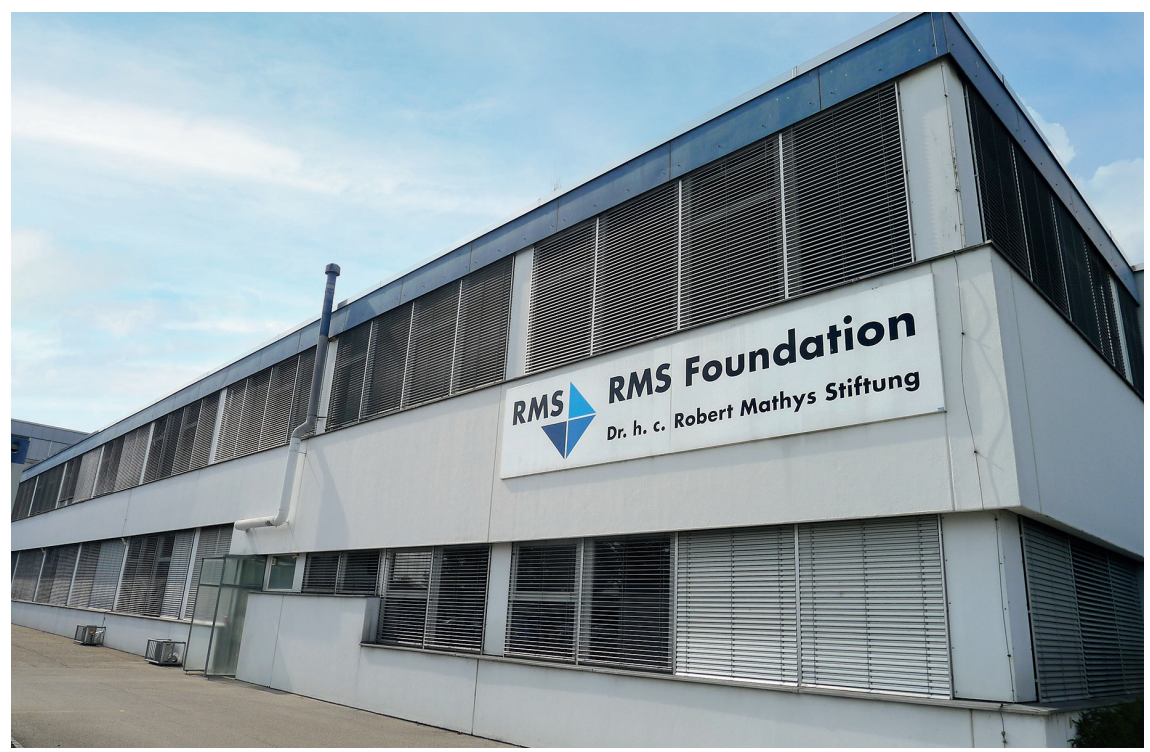

Die RMS Foundation in Bettlach

(C) MATHYS AG, Bettlach

2004 der Verkauf der Osteosynthese-Abteilung, die damals 90 Prozent des Umsatzes generierte, an Synthes-Stratec (siehe S. 30). Dieser Entscheid war aus verschiedenen Gründen sinnvoll. Die Grössenverhältnisse hatten sich im internationalen Rahmen, auch ausserhalb der AO, derart verschoben, dass Mathys in der Traumatologie nur noch mit Mühe mithalten konnte. Ziemlich genau zehn Jahre später bestätigte Urs Schenker, zwischen 1997 und 2004 Mitglied des Verwaltungsrats der Mathys Medizinaltechnik AG, diese Einschätzung, als er auf den Verkauf von Synthes an J \& J einging: «Dass der Verkauf an Synthes der richtige Schritt war, zeigte sich im Übrigen Jahre später, als Synthes selbst in Johnson \& Johnson aufging - der Osteosynthese-Markt erforderte noch grössere Unternehmen. Innovation und Kostenführerschaft, die früher auch in kleineren und mittleren Unternehmen erreicht wurden, konnten nun nur noch mit Grössenvorteilen erkämpft werden - Dimensionen, denen nicht einmal Synthes mehr gerecht wurde.»123 Damit ist gleich auch angedeutet, dass Synthes am 14. Juni 2012 vom US-Medizinkonzern J \& J für 19,7 Mrd. USD übernommen und mit dessen Abteilung De Puy zu De Puy Synthes vereinigt wurde. ${ }^{124}$

123 Schenker, Urs: Robert Mathys - Führung der Mathys Medizinaltechnik AG in der Jahrhundertwende, in: RMS Foundation (Hrsg.), Engagement in zweiter Generation, S.16.

124 Jeannet, Leading a Surgical Revolution, S. 303 und Feldges, Dominik: Der Unmut nimmt in der Synthes-Belegschaft zu, in: Finanz und Wirtschaft 19.12.2012 
Zudem wurde die Zusammenarbeit als Lizenzpartner mit der AO auch aus kartellrechtlichen Gründen mehr und mehr problematisch. Schliesslich ermöglichte der Verkaufspreis von 1,5 Mrd. CHF den Ausbau und die erfolgreiche Weiterführung der Orthopädie (künstlicher Gelenkersatz), die ab 1997 von Hugo Mathys, dem Bruder von Robert Mathys jun., geleitet wurde. ${ }^{125}$ Diese Option erwies sich als richtig, was sich unter anderem in der Beschäftigtenentwicklung während der folgenden Jahre zeigte. Unmittelbar nach dem Verkauf zählte die Firma insgesamt noch 329 Mitarbeitende, davon 183 in der Schweiz. Nur fünf Jahre später hatte sie diese Zahl bereits wieder auf 476 bzw. 280 gesteigert. ${ }^{126}$ Ihr Profil stellt sie dabei selbstbewusst zur Schau: «Seit 2003 fokussiert Mathys ausschliesslich auf Entwicklung, Produktion und Vertrieb von Implantaten für den künstlichen Gelenkersatz in den Bereichen Hüfte, Knie und Schulter sowie synthetisches Knochenmaterial.» ${ }^{127}$ Das Selbstbewusstsein kommt nicht von ungefähr, figurierte Mathys 2013 doch unter den weltweit zehn grössten Gelenkersatzanbietern. Auch in dieser Erfolgswelle blieb (und bleibt) Mathys seinen traditionellen Grundsätzen treu. Die Firma baut auf hoch qualifizierte Arbeitskräfte und verzichtet deshalb auf Produktionsverlagerungen in Billiglohnländer. ${ }^{128}$ Die sorgfältige Produkteentwicklung hat nach wie vor einen hohen Stellenwert, wie folgende Eigenwerbung verdeutlicht: «Die Firma investiert in die Entwicklung eines Produkts über mehrere Jahre. Das muss man durchhalten, so hat man ein im Markt langfristig akzeptiertes Produkt.» ${ }^{129}$ Entsprechend der Tradition des Familienunternehmens ist bis heute ein Börsengang kein Thema. Der Aufstieg hält bis in die jüngste Zeit an. Nachdem die Mathys AG 2013 in die Sportchirurgie eingestiegen war, erhielt sie im August 2018 den «Swiss Excellence Product Award» für das Produkt «Ligamys», das die Regenerierung frisch gerissener Kreuzbänder erlaubt. ${ }^{130}$

Ein umfassendes Bild über die Entwicklung der Firma sowie jene der RMS Foundation liefern die Beschäftigungszahlen.

Siehe Anhang 3: Beschäftigte Mathys AG 1955 - 2017, S. 111

Siehe Anhang 4: Beschäftigte RMS Foundation Bettlach, S. 112

\footnotetext{
125 Schenker, Urs: Robert Mathys, in: RMS Foundation (Hrsg.), Engagement in zweiter Generation, S.15-16 und Mathys, Schlossern für die Gesundheit, in: Betschon/Betschon/Lindecker/Schlachter, Ingenieure bauen die Schweiz, S. 431.

126 Angaben von Robert Mathys jun.

127 Eigenwerbung Mathys, in: TOP 100, November 2015, S. 21.

128 Wittwer, Judith: Durch Mark und Bein, in: Handelszeitung 9.1.2014.

129 Eigenwerbung Mathys, in: TOP 100, November 2009, S.15.

130 Medienmitteilung Mathys 28.8.2018.
} 


\subsection{Synthes: Vom Aussenseiter zum Grosskonzern}

Nach der Gründung im Jahre 1974 nannte sich das Unternehmen Synthes USA, im Anschluss an die Fusion mit Stratec Medical 1999 firmierte es als Synthes-Stratec, Inc. und nach der Übernahme der Mathys AG im April 2004 als Synthes, Inc. Seit 2012, als die Firma an Johnson \& Johnson (J \& J) verkauft wurde, heisst sie De Puy Synthes. Diese Namensänderungen spiegeln jeweils Meilensteine in der kurzen Unternehmensgeschichte wider. Wir benutzen nachstehend den Namen «Synthes».

In einem kurz vor der Übernahme durch J \& J publizierten Flyer hält Synthes fest: «Unsere Erfolgsgeschichte geht auf zwei Schweizer Unternehmen zurück: Mathys Medizinaltechnik AG in Bettlach und das Institut Straumann in Waldenburg. ${ }^{131}$ Diese Aussage ist ebenso kurz wie zutreffend. Die Bedeutung der schweizerischen Wurzeln kommt auch darin zum Ausdruck, dass Solothurn/Zuchwil weiterhin der Hauptsitz für Europa, den Nahen Osten und Afrika ist. Bis zur Übernahme durch J\&J befanden sich in der Schweiz auch die Produktionsentwicklungszentren für die Geschäftsfelder der Traumatologie, der Wirbelsäule und von Cranio-Maxillofacial.

Bis Mitte der 1980er-Jahre waren AO-Produkte in den USA wenig verbreitet, zumal sich die Vorbehalte gegenüber der Technik der Osteosynthese hartnäckig hielten. Nachdem die Firma Straumann auf dem schwierigen Boden nicht hatte Fuss fassen können, bauten einige amerikanische Chirurgen Synthes USA auf. Durch Vermittlung des Basler Chirurgen Martin Allgöwer übernahm Hansjörg Wyss 1977 die Firma. Unter seiner Führung steigerte Synthes USA zwischen 1977 und 1999 den Umsatz von 3,5 Mio. USD auf über 500 Mio. USD. Drei Ziele waren für sein (erfolgreiches) Handeln wegleitend: Gründung einer US-Produktionsstätte (1979 in Monument, Colorado erfüllt), regelmässige Schulungen in den USA und Aufbau einer Verkaufsinfrastruktur. ${ }^{132}$

Wie erwähnt, geht ein Pfeiler von Synthes auf die Firma Straumann in Waldenburg zurück. Nach dem plötzlichen Tod von Fritz Straumann erwarb der Liestaler Rudolf Maag 1989 den Löwenanteil der Firma durch ein Management-Buy-out in Höhe von 120 Mio. CHF. Der neu unter dem Namen Stratec Medical auftretende Betrieb umfasste ungefähr 75 Prozent des ehemaligen Stammhauses, während die restlichen Unternehmensteile (Uhren, Dentalprothetik, Elektronik) bei der Familie Straumann verblieben. Nachdem die AO 1989 das noch von Fritz Straumann entwickelte Titan als Material für Implantate genehmigt hatte, verdreifachte Stratec Medical den Umsatz innert fünf Jahren. Neue Fabriken wurden in Oberdorf (BL) und Mezzovico (TI) errichtet, in Hägendorf (SO) wurde ein bestehender

131 Synthes, Synthes in der Schweiz.

132 Schlich, Surgery, Science and Industry, S.180-188. 
Betrieb übernommen. Ab diesem Zeitpunkt gab es neben Mathys Medizinaltechnik AG mit Stratec Medical im Kanton Solothurn einen weiteren Medtech-Grossbetrieb. 1992 wurde die Produktion auf die Bereiche «Gesicht» und «Wirbelsäule» sowie auf Antriebsmaschinen und Sterilisationsbehälter ausgeweitet. Neben dem Einstieg in den Markt für "Computerunterstützte Systeme in der Medizin» übernahm die Firma 1995 die Tochtergesellschaft Synthes GmbH in Umkirch (D) sowie die auf künstliche Hüftgelenke spezialisierte Pantitan AG in Wien und errichtete in Oberdorf (BL) ihren Konzernhauptsitz. ${ }^{133} \mathrm{Zu}$ diesem Zeitpunkt verfügte Stratec Medical über 16 operative Betriebe mit 730 Mitarbeitern. Der Jahresumsatz betrug 185 Mio. CHF bei einem Reingewinn von 25,7 Mio. CHF. Der Export umfasste 90 Prozent der Verkäufe. Mit 83 Prozent des Umsatzes war die Osteosynthese nach wie vor das Kerngeschäft; Implantate und Instrumente machten den Löwenanteil aus. Daneben hatte Stratec-Medical ein kleineres Standbein in der Prothetik, wo sie, wie Mathys, die Firma Protek belieferte. 1996 erfolgte der Börsengang.

Bis zur Fusion mit Synthes 1999 hatte das Unternehmen mit 265 Mio. CHF den Umsatz seit dem Management-Buy-out beinahe verfünffacht. ${ }^{134}$ Diese Expansion kann als Folge eines allgemeinen Entwicklungsschubs in der Medizintechnik gesehen werden, der ab den 1970er-Jahren einsetzte, begann doch «... weltweit die Einführung von künstlichen Hüft- und Kniegelenken und die chirurgische Behandlung von Knochenbrüchen aller Art». ${ }^{135}$ Mit der Fusion von Stratec Medical und Synthes 1999 wurde die neu gebildete Synthes-Stratec, Inc. die Nummer 1 in der Welt bei der Osteosynthese. Innerhalb Europas nahm sie zudem eine beachtliche Position in der Orthopädie ein. Sie deckte die Märkte in den USA, in Lateinamerika und in wichtigen Teilen Europas ab. ${ }^{136}$ Die neu gebildete Firma ergab durchaus Sinn, wie Hansjörg Wyss im Geschäftsbericht der Synthes-Stratec 1999 betonte: «Der Zusammenschluss führt zwei Unternehmen zusammen, die über gemeinsame Wurzeln verfügen, in unterschiedlichen geografischen Regionen eine führende Stellung einnehmen und während vieler Jahre erfolgreich zusammengearbeitet haben.»137 Was bei der Fusion kaum erwähnt wurde, war der Umstand, dass es sich um zwei ungleiche Partner handelte. Hansjörg Wyss besass 61,5 Prozent, Rudolf Maag 13,7 Prozent der Aktien. Der Rest war frei flottierend. ${ }^{138}$ Das einseitige Kräfteverhältnis wirkte sich knapp ein Jahr später nach der ersten Meinungsverschiedenheit aus. Während Maag das Prothetik-Geschäft beibehalten wollte, befürwortete Wyss des-

133 Stratec Holding AG, Verkaufsprospekt.

134 Furler, Stratec, S. 5-6 und 10 und Jeannet, Leading a Surgical Revolution, S. 377.

135 Wyss, Hansjörg Wyss, S. 96.

136 Gachnang, Unternehmerportraits-Medizinaltechnik, S. 2O-21.

137 Synthes-Stratec, Geschäftsbericht 1999, S. 9.

138 Blum, Synthes-Stratec, S. 9. 
sen Verkauf. Folge dieser Divergenz: Wyss setzte sich durch, das Prothetik- Geschäft wurde an Biomet Merck verkauft, Maag zog sich aus dem Unternehmen zurück. ${ }^{139}$ Ungeachtet dieser personellen Auseinandersetzung, entwickelte sich das neue Unternehmen Synthes-Stratec ausgezeichnet. «Überraschend kräftiges Wachstum bei Synthes-Stratec», titelte die Neue Zürcher Zeitung stellvertretend für viele ähnliche Einschätzungen. ${ }^{140}$ Bereits unmittelbar nach der Fusion stellte die Bank Julius Bär fest, dass der Umsatz bei einer Gesamtbeschäftigtenzahl von 2600 um das 3,3-Fache gestiegen war. Dazu trage die ehemalige Osteosynthese von Synthes 70 Prozent, jene von Stratec-Medical 24 Prozent und die ebenfalls von Stratec-Medical eingebrachte (und nach einem Jahr verkaufte) Orthopädie 6 Prozent bei. Als Weltmarktleader habe Synthes-Stratec bei der Osteosynthese in den USA einen Marktanteil von 30 Prozent, in Europa sogar von 36 Prozent. Das neue Unternehmen war nun auch der wichtigste Lizenznehmer der Synthes Chur AG, indem es zwei Drittel der Lizenzgebühren - gegenüber einem Drittel der Mathys AG - einbezahlte. ${ }^{141} \mathrm{Im}$ Geschäftsbericht 2000 wies Synthes-Stratec 3140 Mitarbeitende und ein Umsatzwachstum von 22 Prozent aus. Die Wachstumsrate der Beschäftigten habe durchschnittlich 12 Prozent jährlich betragen. ${ }^{142}$ Ein Jahr später wurde ein Umsatzwachstum von 17,5 Prozent und eine Umsatzrendite von 24,3 Prozent erzielt, nachdem sich die Firma ausschliesslich auf die Osteosynthese fokussiert hatte. ${ }^{143}$ Der Geschäftsbericht 2002 hebt hervor, dass der Konzern zehn Millionen Artikel führe und dass jeder der 3803 Mitarbeiter mit 161000 CHF zum Betriebsergebnis beitrage. ${ }^{144}$ Zwischen 1999 und 2003 steigerte Synthes-Stratec den Umsatz von 695 Mio. USD auf 1229 Mio. USD, wobei drei Viertel dieser Summe auf dem US-Markt generiert wurden. Die Beschäftigtenzahl erreichte mittlerweile die 40ooer-Grenze. ${ }^{145}$

Die bereits erwähnte Übernahme der Osteosynthese-Abteilung von Mathys war der nächste und entscheidende Schritt. Bei Mathys verblieb lediglich die Prothetik. Abgesehen von zusätzlich rund 1900 Beschäftigten (davon 900 in Bettlach), war Synthes nun auf dem gesamten Weltmarkt vertreten, da Mathys und Synthes-Stratec mit nahezu identischen Produkten der Marke Synthes bloss in unterschiedlichen Märkten agierten. Mit der Übernahme verlagerte Synthes den schweizerischen Schwerpunkt an den Jurasüdfuss, wo eine Art Medtech-Cluster entstand. ${ }^{146}$

139 Städeli, Markus: Synthes-Stratec: Wyss auf dem Höhepunkt, in: Handelszeitung 19.8.2003.

140 I.M.: Überraschend kräftiges Wachstum bei Synthes-Stratec, in: Neue Zürcher Zeitung 11.8.200o.

141 Julius Bär, Synthes-Stratec, S. 4-5 und 11.

142 Synthes-Stratec, Geschäftsbericht 2000, S.7.

143 Synthes-Stratec, Geschäftsbericht 2001, S. 6-7.

144 Synthes-Stratec, Geschäftsbericht 2002, S. 6.

145 Jeannet, Leading a Surgical Revolution, S.304-305.

146 Pedroitto, Franziska: Synthes-Stratec prescht der Konkurrenz davon, in: Finanz und Wirtschaft 16.8.2003. 
Die nahezu optimale Synergie illustriert ein Titel, den die NZZ bei der Ankündigung der Fusion platzierte: «Das perfekte Paar in der Medizintechnik». ${ }^{147}$

Selbst eine Klage gegen die von Synthes in den USA übernommene Knochenersatzpaste Norian konnte den Erfolg nicht aufhalten. Nachdem drei Patienten nach Verwendung dieses in der Wirbelsäulenchirurgie angewandten Materials gestorben waren, bezahlte Synthes eine Busse von 23 Mio. USD und nahm das Produkt selbstredend vom Markt. Zudem wurden vier Synthes-Kaderleute zu Gefängnisstrafen verurteilt. ${ }^{148}$ Dem Unternehmenserfolg, der sich nach der Fusion mit Mathys gar noch akzentuieren sollte, tat dieser Vorfall aber keinen Abbruch.

Bevor wir diesen Zeitabschnitt genauer betrachten, richten wir das Augenmerk auf das Selbstverständnis von Synthes und die Person von Hansjörg Wyss. Die Norian-Episode zeigte auf, wie stark sich Synthes in einem Zielkonflikt zwischen Profitmaximierung, Konkurrenzdruck und Patientennutzen befand (und befindet). ${ }^{149}$ Ungeachtet dieses Spannungsfeldes, strahlte der Konzern ein starkes Selbstbewusstsein aus, wie in einer Selbstdarstellung kurz vor der Übernahme durch J\&J klar zum Ausdruck kommt: «Synthes has set the standard for treating fractures. We are the world leader in traumatology, we are among the top companies for spinal devices and we are in the leading position in the cranio-maxillofacial business. $\rangle^{150}$ Zusammen mit der AO, fährt Synthes fort, sei man führend in Forschung und Entwicklung. Die ganze Unternehmensphilosophie und -praxis basiere auf folgenden sieben Prinzipien: Patientenorientierung, Fokussierung auf den Chirurgen, Innovation, Qualität, Schulung, Partnerschaft und Integrität. ${ }^{151}$ Bereits zu Beginn seiner Aktivitäten legte Wyss Wert auf Schweizer Methodik und Logik, d.h. auf Kurse, Workshops und Netzwerkbildung («Fellowship»). Mit den von der AO durchgeführten Schulungen für Ärzte und Operationspersonal wies Synthes ein klares Unterscheidungsmerkmal gegenüber der Konkurrenz auf. ${ }^{152}$

Im Zentrum stand bei Synthes stets die Qualität der menschlichen Arbeitskraft. Folgende Aussage bringt diese Grundhaltung auf den Punkt: «Unser zukünftiger Erfolg wird davon abhängen, dass wir die allerbesten Leute gewinnen, fördern und halten ... $»^{153}$ Besonderes Gewicht wurde auf hoch qualifizierte Mitarbeitende im Aussendienst gelegt. Entsprechend wurde deren Anteil an der gesamten Beschäftigtenzahl seit der Übernahme von Mathys sukzessive erhöht. 2008 waren 30 Pro-

\footnotetext{
147 cei: Das perfekte Paar in der Medizintechnik, in: Neue Zürcher Zeitung 14.8.2003.

148 Wyss, Hansjörg Wyss, S.101-104.

149 Ebd., S.101.

150 Synthes, Dedicated to Health.

151 Ebd.

152 Schlich, Surgery, Science and Industry, S.194.

153 Synthes, Geschäftsbericht 2005, S. 5.
} 
zent der Synthes-Angestellten im Aussendienst tätig. Diese waren nicht bloss Verkäufer, sondern hoch spezialisierte Berater, die sich ihr Fachwissen in Schulungen und bei der Lancierung eines neuen Produktes sogar durch Assistenz bei Operationen holten. ${ }^{154}$ Unmissverständlich äussert sich der Geschäftsbericht 2008: «Wir ... verfügen ... weiterhin über die am besten geschulten, engagiertesten und am höchsten spezialisierten Aussendienstmitarbeitenden innerhalb der Branche.»1155

Robert Frigg, ab 2004 Chief Technology bei Synthes, Entwickler von 250 Patenten und während vieler Jahre engster Mitarbeiter von Hansjörg Wyss, bestätigt diese Aussage, indem er betont, dass die menschliche Arbeitskraft für den SynthesChef stets im Zentrum stand: «Wyss schätzte Handwerk, Können und Wissen und baute Netzwerke auf. Die Aussendienstmitarbeiter waren deshalb bei den Operationen anwesend. Das machte er mantramässig, und das war sein Erfolg. ${ }^{156}$ Er war ein «Patron der alten Schule», viel eher Ingenieur als Manager, «der für seine Produkte lebte». Diese stünden im Vordergrund, nicht Börsenzahlen, konterte er jeweils Anfragen von Journalisten, die genauere Prognosen über die Kursentwicklung in Erfahrung bringen wollten. Hedi Wyss, seine Schwester, unterstreicht diese Einschätzung, wenn sie festhält, für ihren Bruder sei es unerlässlich gewesen, dass Synthes-Produkte die besten und «in aller Leute Mund» seien. Folgerichtig sei er ein akribischer, ins Operative eingreifender Chef gewesen, im Umgang mitunter rechthaberisch, cholerisch - ein Machtmensch. ${ }^{157}$ Der Erfolg gab ihm recht, denn: «Zu Wyss' bemerkenswerten Fähigkeiten gehört, vorausschauen zu können, zu wissen, was wichtig ist und worauf es ankommt, damit ein Plan gelingt. ${ }^{158}$ Die Richtigkeit seiner Entscheide und Vorgehensweise zeigt sich auch in messbaren Fakten. Beim Verkauf von Synthes an J\&J besass Wyss mehr als 50 Prozent der Aktien. Zudem wurde er Ehrendoktor der Universitäten Basel (2004) und Salzburg (2005) und der Clemson University. ${ }^{159}$ So verwundert es nicht, dass Wyss auch nach dem Verkauf von Synthes nicht von der Medizintechnik ablassen konnte. Ende 2012 kaufte er in Bettlach die auf Präzisionsmechanik spezialisierte Firma Startech GmbH. Nachdem De Puy Synthes die Innovationsgruppe in Zuchwil geschlossen hatte, gelangten mehrere Medtech-Experten in den Betrieb, der sich seit Anfang 2016 41medical AG nennt, von Robert Frigg geleitet wird und zu einem wichtigen Entwickler und Zulieferer für die Medizintechnik geworden ist. ${ }^{160}$

\footnotetext{
154 Pictet, Synthes-Stratec, S.19.

155 Synthes, Geschäftsbericht 2008, S.2.

156 Gespräch mit Robert Frigg.

157 Städeli, Synthes-Stratec, in: Handelszeitung 19.8.2003 und Wyss, Hansjörg Wyss, S. 108-109.

158 N.N.: Der Milliardär aus Bern-Weissenstein, in: Berner Zeitung 17.5.2014.

159 Jeannet, Leading a Surgical Revolution, S. 377 und Synthes Annual Report 2010, CG4.

160 Leibundgut, Barbara: Wie ehemalige Synthes-Mitarbeiter ihr neues Medtech-Unternehmen aufbauten, in: Solothurner Zeitung 19.11.2017.
} 
Vor dem Hintergrund des Selbstverständnisses von Synthes und der Persönlichkeit von Hansjörg Wyss lässt sich die nochmals sprunghafte Entwicklung, die nach der Übernahme von Mathys einsetzte, besser einordnen. Die Produkteentwicklung wurde zusammengelegt, bei gleichzeitiger Verstärkung der weltweiten Verkaufsteams. Die Synergieeffekte führten dazu, dass Synthes gleich im ersten Jahr danach, also 2005, mit rund 7600 Mitarbeitenden (davon 2200 in der Schweiz) erstmals einen Gesamtumsatz von über 2 Mrd. USD erwirtschaftete, bei einer Steigerung von 16,8 Prozent. ${ }^{161}$ Die Machtkonzentration war mit ein Grund für die am 15. März 2006 erfolgte Vertragsänderung mit der Synthes Chur AG, die die Patentrechte auf Synthes übertrug (siehe S. 30). ${ }^{162}$ Trotz der Dimension eines globalen Konzerns bewahrte Wyss in der Schweiz die Struktur mit relativ kleinen Einheiten, da diese effizient seien. Zudem befanden sich diese untereinander in naher Distanz, sodass eine Art Synthes-Campus entstand. ${ }^{163}$

Die Übertragung der Patentrechte auf Synthes wurde 2006 von der Universität Bern angefochten, mit der Begründung, sie sei an der Wirbelsäulenchirurgie mitbeteiligt gewesen. Fünf Jahre später meinte der inzwischen emeritierte Berner Professor Max Aebi: «Es darf nicht sein, dass die Universitäten die ganze Forschungsstruktur zur Verfügung stellen und dann nur private Konzerne wie Synthes abkassieren. ${ }^{164}$ Schliesslich ist aber die Universität mit ihrer Klage vor Gericht abgeblitzt. ${ }^{165}$ Dieser Gerichtsfall war gewissermassen und indirekt der Schlusspunkt einer Kontroverse zwischen Maurice E. Müller und Hansjörg Wyss, die bis in die 199oer-Jahre zurückreicht. Müller hatte damals Wyss eine Abkehr von den AO-Prinzipien und eine Kommerzialisierung vorgeworfen. Dieser erwiderte, die Komplexität und Grösse des Marktes seien mit den schwerfälligen Entscheidungsprozessen bei der AO nicht mehr kompatibel. ${ }^{166}$ Ungeachtet solcher Reibungsflächen, war Synthes ganz einfach nicht aufzuhalten. Der Sturmlauf spiegelt sich in der Umsatzentwicklung von 0,9 Mrd. USD im Jahre 2001 auf 3,687 Mrd. USD 2010 wider, dies bei einer Gewinnmarge von 24,6 Prozent. Wachstumstreiber waren dabei die USA, wo 2010 der Umsatz 2,14 Mrd. USD ausmachte, gegenüber 850,2 Mio. USD in Europa und 424,4 Mio. USD in Asien/Pazifik. Auf die restlichen Regionen entfielen 258,2 Mio. USD. Es erstaunt deshalb nicht, dass Asien als Wachstumsmarkt definiert und der

\footnotetext{
161 Breiding/Schwarz, Wirtschaftswunder Schweiz, S. 266 und Synthes, Geschäftsbericht 2005, S.4.

162 Helvea, Synthes. Nailing Success, S. 5 und 11.

163 Jeannet, Leading a Surgical Revolution, S. 310 und 366.

164 Wissmann, Reto: Im Synthes-Patentstreit wirft die Uni der Gegnerin Verzögerungstaktik vor,

in: Der Bund 23.4.2011.

165 Jeannet, Leading a Surgical Revolution, S. 300.

166 N.N.: Unbekannter Milliardär, in: Neue Zürcher Zeitung 7.5.2006.
} 
Standort China im selben Jahr ausgebaut wurde. ${ }^{167}$ Vergleichbar zu den Umsätzen stiegen auch die Beschäftigtenzahlen. Diese kletterten von 6711 (2004), 7627 (2005), 8451 (2006), 9070 (2007), 9947 (2008), 10705 (2009) auf 11426 im Jahre 2010. ${ }^{168}$ Aufschlussreich ist auch die Mitarbeiterstruktur, aufgeteilt nach Funktionsgruppen: Fertigung/Produktion 43 Prozent, Aussendienst 27 Prozent, Produkteentwicklung 9 Prozent, sonstiges Fachpersonal 27 Prozent. ${ }^{169}$ Weltweit hatte Synthes im Traumabereich 2009 einen Marktanteil von über 50 Prozent. Diese Position hielt der Konzern auch zwei Jahre später, vor der Übernahme durch J \& J. ${ }^{170}$

Gemäss Robert Frigg hatte Hansjörg Wyss auch etwas Glück, dass er Synthes im bestmöglichen Moment unter so guten Bedingungen zu einem Preis von 19,7 Mrd. USD an J\& J verkaufen konnte (siehe S. 21). ${ }^{171}$ Gesamthaft gesehen, war J \& J mit einem Umsatz von 61,6 Mrd. USD und total 115000 Mitarbeitenden (Zahlen von 2010) gegenüber Synthes mit 11426 Angestellten und einem Umsatz von knapp 3,7 Mrd. USD ein Riese. Bezüglich der Unfallchirurgie waren die Grössenverhältnisse jedoch gerade umgekehrt. Der Weltmarktanteil betrug bei Synthes 50 Prozent, bei De Puy 4 Prozent. Bei den Wirbelsäulenimplantaten partizipierten die beiden Firmen mit je 10-15 Prozent. ${ }^{172}$ Vergleichbar waren zum Zeitpunkt des Fusionsbeschlusses die Mitarbeitenden in der Schweiz: J \& J mit rund 3000, Synthes mit 2800, davon 1600 im Kanton Solothurn. ${ }^{173}$ Mit der Namensbildung De Puy Synthes war Synthes als Firmenbezeichnung weiterhin präsent. Die Fusion vom 14. Juni 2012 war wohl vorbereitet. Das zeigt sich allein schon darin, dass seit deren Ankündigung am 27. April 2011 über ein Jahr verstrichen war. Mehrheitlich waren sich die Fachleute darin einig, dass sich am Standort Schweiz dank hoher Produktivität, moderner Anlagen, optimaler Fabrikationsverfahren und weiterhin wachsendem Traumamarkt nicht allzu viel ändern würde. ${ }^{174}$ Sowohl die Aktionäre wie auch Wyss, der J\& J aufgrund

167 Synthes, Synthes. Dedicated to Health, Palm, Carla: Synthes muss sich strecken, in: Finanz und Wirtschaft 4.9.2010 und Jeannet, Leading a Surgical Revolution, S.305.

168 Synthes, Geschäftsbericht 2007, S.7, Synthes, Geschäftsbericht 2008, S. 2, Synthes, Geschäftsbericht 2009, S. 2 und Synthes, Annual Report 2010, S.13.

169 Synthes, Geschäftsbericht 2008, S.27.

170 Synthes, Geschäftsbericht 2009, BR 2 und Feldges, Dominik: Synthes verliert Elan, in: Finanz und Wirtschaft 18.2.2012.

171 Wyss, Hansjörg Wyss, S.180.

172 Müller, Giorgio V.: Der Medtech-Standort Schweiz hat internationales Renommee, in: Neue Zürcher Zeitung 28.4.2011 und Feldges, Dominik: Der Unmut nimmt in der Synthes-Belegschaft zu, in: Finanz und Wirtschaft 19.12.2012.

173 Müller, Giorgio V: Synthes bleibt der Schweiz treu, in: Neue Zürcher Zeitung 13.6.2012 und Boder, Willy: Johnson et Johnson absorbe Synthes pour devenir le leader Mondial de l'ostéosynthèse, in: Le Temps 28.4.2011.

174 Müller, Giorgio V: Synthes bleibt der Schweiz treu, in: Neue Zürcher Zeitung 13.6.2012 und Schnyder, Stefan: «Wie die meisten Reichen zahle ich zu wenig Steuern«, Interview mit Hansjörg Wyss, in: Berner Zeitung 19.5.2011. 
deren dezentraler Entscheidungsstruktur als "gutes Heim» für Synthes bezeichnete, standen dem Verkauf positiv gegenüber. Robert Mathys jun. teilte diese Einschätzung, indem er der Übernahme durch J\&J eine stabilisierende Wirkung beimass. Bei Wyss mag die wohlwollende Beurteilung auch damit zusammengehängt haben, dass er mit einem Anteil von 8 Prozent grösster Hauptaktionär bei J \& J wurde. ${ }^{175}$ Lediglich die Westschweizer Zeitung Le Temps äusserte sich kritisch, da die Fusion zu einem Abfluss des Wissens, des Know-how und der Forschung hinaus aus der Schweiz führen werde. ${ }^{176}$ Der letzte Punkt sollte sich zwei Jahre später bewahrheiten, indem die Forschungs- und Innovationsabteilung für neue Implantate in Zuchwil aufgelöst wurde. Der Beschäftigungslevel wurde von J \& J ungefähr gehalten. Die Abteilung De Puy Synthes zählte 2015 in der Schweiz 2900 Angestellte, davon über 2000 im Kanton Solothurn. Sie war weltweit das grösste und umfassendste Orthopädieunternehmen innerhalb des - ebenfalls weltweit - grössten Gesundheitsunternehmens. ${ }^{177}$ Ende 2017 hielt Michelle Brennan, Verantwortliche von De Puy Synthes, fest: «Seit 2012 hat die Zahl der Arbeitsplätze von De Puy Synthes in der Schweiz um 5 Prozent zugenommen.»178 Aufschlussreich sind die Beschäftigtenzahlen an den Solothurner Standorten. In Solothurn/Zuchwil, nach wie vor Hauptsitz für Europa, Mittlerer Osten und Afrika, arbeiten rund 700 Personen, in Bettlach 370, in Hägendorf 340, in Grenchen 320, in Balsthal 110. Wieweit die gestaffelte Akquisition der beiden Werke Grenchen und Bettlach durch den US-Auftragsfertiger Jabil, beschlossen im Juli 2018, Auswirkungen auf die Beschäftigtenzahl haben wird, bleibt einstweilen offen. ${ }^{179}$

Alles in allem überwiegt nach der Übernahme von Synthes durch J \& J die Kontinuität. Vorschriften bezüglich Forschung, Entwicklung, Fertigung, Verkauf und Transparenz werden strikt befolgt. Die Zielgruppen, bestehend aus Ärzten, Krankenkassen, Materialverantwortlichen, Krankenhäusern, Einkaufsgemeinschaften und Managed-Care-Organisationen, sowie die Lieferanten und Regulierungsbehörden sollen gemäss Global Code von J \& J optimal informiert werden. ${ }^{180}$ Dass De

175 Toggweiler, Andreas: Schnittstelle zwischen Forschung und Industrie, in: SO Economy 16.6.2011, S.11, Müller, Giorgio V.: «Wir haben ein gutes Heim für Synthes gefunden«, in: Neue Zürcher Zeitung 11.6.2011 und Mathys, Robert: «Kostensenkungen sind ein Thema«, in: SO Economy 16.6.2011.

176 Boder, Willy: Le savoir-faire suisse s'étiole, in: Le Temps 28.4.2011.

177 Schaible, Franz: Europa-Hauptsitz des grössten Medtechkonzerns der Welt bleibt in Zuchwil, in: Solothurner Zeitung 2.9.2016, Eigenwerbung, in: TOP 100, November 2015, S. 2 und Eigenwerbung, in: TOP 100 November 2016, S. 2 und 5.

178 Müller, Giorgio V.: "Seit der Übernahme von Synthes hat Johnson \& Johnson in der Schweiz Stellen geschaffen««, in: Neue Zürcher Zeitung 5.11.2017.

179 Mathys, Urs: Auch Synthes-Standorte Balsthal und Hägendorf sollen an Jabil gehen, in: Solothurner Zeitung 13.9.2018 und Müller, Giorgio V.: Schweizer Mitarbeiter von J \& J wechseln Firma, in: Neue Zürcher Zeitung 20.7.2018.

180 De Puy Synthes, Integrität in Aktion, S. 3 und 5. 


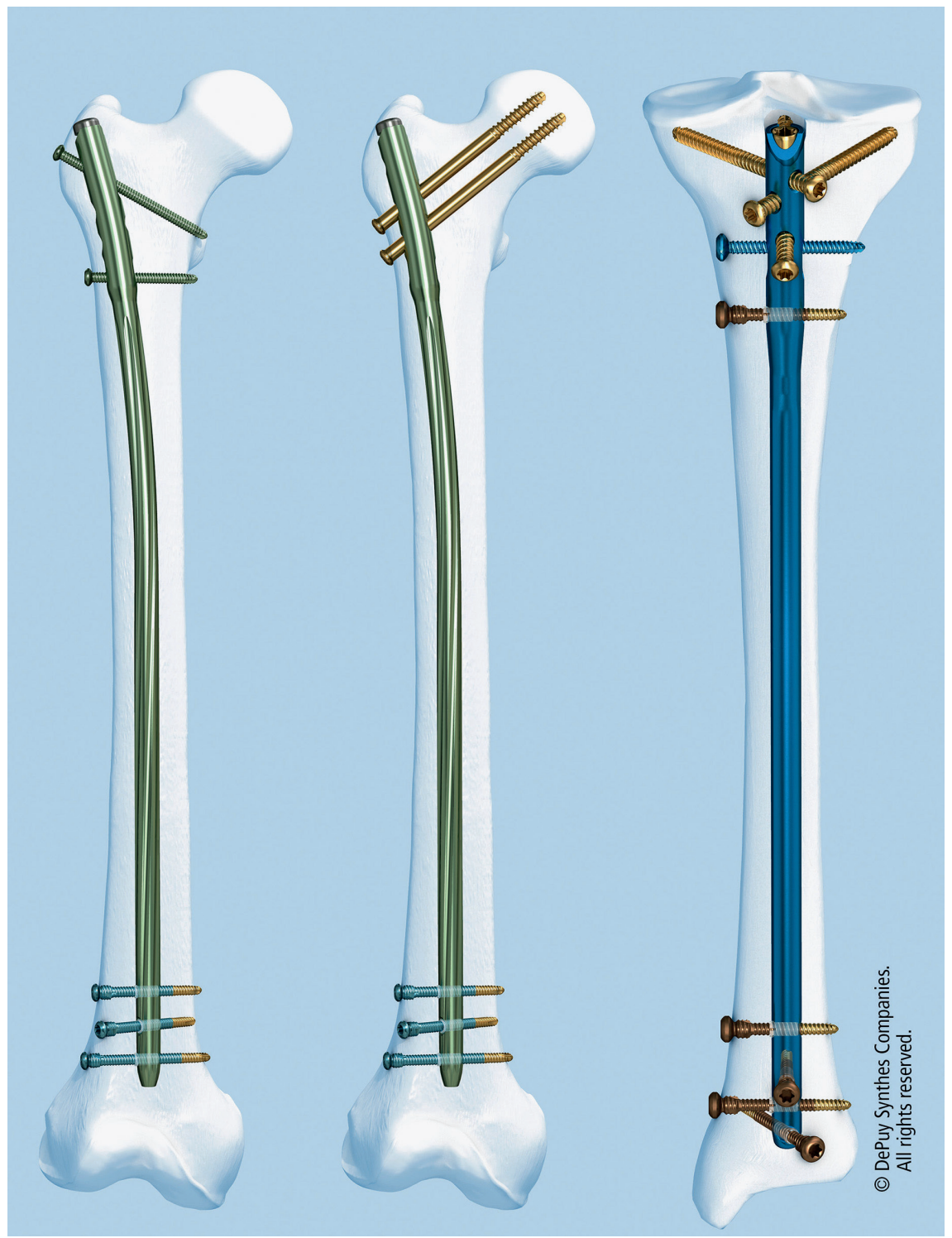

Verriegelungsmarknägel: Anwendungen im Oberschenkel (links und Mitte) und Anwendung im Unterschenkel (rechts)

DePuy Synthes Companies. All rigths reserved 
Puy Synthes die vertraglich festgelegte Zusammenarbeit mit der AO-Stiftung fortsetzt, wurde bereits erwähnt (siehe S.30-31), dies auch deshalb, weil die Kurse der AO um einiges kostengünstiger sind als vergleichbare Angebote. ${ }^{181}$ Kontinuität verrät schliesslich auch die Aussage von Michelle Brennan zu den Unternehmenszielen: «Innovative Produkte, Dienstleistungen und Prozesse dienen nicht nur dazu, bessere medizinische Resultate für die Patienten zu erzielen. Ziel ist auch, die Aufenthaltsdauer im Spital zu verkürzen, Infektionen und Komplikationen zu vermeiden und damit letztlich die Folgekosten zu minimieren.»182

Die in diesem Kapitel aufgeführten Beschäftigungszahlen betreffen mehrheitlich das ganze Unternehmen Synthes bzw. ab 2012 De Puy Synthes.

Siehe Anhang 5: Beschäftigte von Synthes im Kanton Solothurn zwischen 2007 und 2018, S. 113

\subsection{Stryker oder Amerika sucht die Schweiz}

Die Stryker GmbH in Selzach geht auf das 1963 von den beiden ehemaligen Straumann-Mitarbeitern Vogt und Karpf gegründete Unternehmen Voka zurück. Ein Jahr später übersiedelte dieses unter dem neuen Namen Osteo von Altreu nach Selzach. Osteo agierte in Konkurrenz zu Mathys und Straumann - ausserhalb der AO. Über ihre Firmengründer baute sie auf dem Know-how der beiden AO-Partner auf. In den ersten Jahren nach ihrer Gründung hatte sie sogar einen gewissen Marktvorteil, da sie sich nicht an den Entwicklungskosten beteiligen musste und dem Genehmigungsverfahren durch die Technische Kommission der AO nicht unterstellt war. Andererseits war sie von den Optimierungen der AO und von Publikationen in Fachzeitschriften ausgeschlossen. ${ }^{183}$ Aus diesem Grunde war die Osteo zwar erfolgreich, blieb aber in ihrer Expansion doch limitiert. Ihre Schwerpunkte waren gemäss Auskunft von Thomas Wahl, bis Mitte 2018 Generalmanager von Stryker Selzach, Knochenersatzmaterialien, Hüft- und Gelenkersatz sowie die Traumatologie. Die Produkte waren weitgehend analog jenen von Synthes, wenn auch mit etwas anderen Akzenten. Eine Pionierrolle übernahm Osteo im Bereich Keramik-Keramik-Systeme. Es handelte sich dabei um Hüftprothesen, bei denen Hüftkopf und Hüftpfanne beide aus Keramik gefertigt waren. Diese neue Entwicklung

181 Jeannet, Leading a Surgical Revolution, S.307.

182 Schaible, Franz: Europa - Hauptsitz des grössten Medtechkonzerns der Welt bleibt in Zuchwil, in: Solothurner Zeitung 2.9.2016.

183 Schlich, Surgery, Science and Industry, S. 63-64. 
entstand in Zusammenarbeit mit Heinz Mittelmeier, Ordinarius für Orthopädie an der Universität Homburg. Zwischen 1986 und 1996 richtete Osteo den Fokus auf Gelenkersatz und Marknagelung. Für Stryker war die Selzacher Firma wegen ihrer Position in der Unfallchirurgie von Interesse. Wahl fasst das Motiv von Stryker kurz und bündig zusammen: «Osteo wurde von Stryker übernommen, weil dieser Konzern neu in die Traumatologie einsteigen wollte.»184

Die Firma Stryker wurde 1946 von Homer Stryker als Orthopedic Frame Company gegründet. Der 1964 in Stryker Corporation umbenannte Konzern deckte die Orthopädie, Neurotechnologie sowie Medizinische Technologie und Ausrüstung ab. Das wichtigste Tätigkeitsfeld der Osteo war für Stryker folglich eine ideale Ergänzung. 2013 zählte Stryker weltweit 21000 Beschäftigte und nahm unter den Medtech-Unternehmen den zehnten Rang ein. ${ }^{185}$ Der Standort Selzach erlebte nach der Integration bei Stryker seine eigentliche Erfolgsgeschichte. Zwei Jahre nach dem Kauf von Osteo übernahm Stryker von Pfizer die ebenfalls in der Traumatologie tätige Howmedica. Obschon diese Firma wesentlich grösser als die ehemalige Osteo war, wurde 2000 die gesamte Platten- und Schraubenproduktion in Selzach konzentriert, nachdem dort die Prothetik eingestellt worden war. Die neue Zweigniederlassung nannte sich fortan Stryker Trauma Selzach AG. Im Jahre 2011 wurden zudem die von Stryker übernommenen Hersteller Memometall SA und Small Bone Innovations ebenfalls nach Selzach transferiert. Allein zwischen 1996 und 2013 erlebte Selzach als klar wichtigster Schweizer Standort von Stryker fünf Ausbauetappen. ${ }^{186}$ Das sprunghafte Wachstum äusserte sich auch in der Beschäftigtenentwicklung. Waren es 1996 bei der Übernahme von Osteo siebzig Beschäftigte, so stieg deren Zahl bis 2003 auf über 300, bis 2013 (fünfzig Jahre nach der Gründung der Osteo) auf rund 500 an. Heute (2018) arbeiten über 600 Personen bei Stryker Selzach, davon drei Viertel in der Produktion und Logistik sowie hundert als Ingenieure in der Forschung und Entwicklung. ${ }^{187}$ Die Produkte werden innerhalb des Konzerns an Zwischenlager in den Niederlanden und in den USA geliefert. Bei der Produktion stützt sich der Standort Selzach auf ein differenziert ausgebautes Netzwerk von Zulieferern in der Region. Gemäss Thomas Wahl werden ungefähr 50 Prozent der Wertschöpfung inhouse generiert. ${ }^{188}$

Schlüssel zum Erfolg war die Konzentration auf die Traumatologie, wie dies

\footnotetext{
184 Gespräch mit Thomas Wahl.

185 Ebd. und www.greaterzuricharea.com.

186 Gespräch mit Thomas Wahl und www.stryker.de > selzach.

187 Gespräch mit Thomas Wahl, Stryker GmbH, Selzach: der Global Player in der Medizintechnik, in: TOP 100, November 2018, S. 17 und Schaible, Franz: Die Firma Stryker ist auf Erfolgskurs, kämpft aber um Fachkräfte, in: Solothurner Zeitung 8.6.2018.

188 Gespräch mit Thomas Wahl.
} 
in der Eigenwerbung deutlich wird: «Stryker Selzach gilt als Spezialist für die Fertigung von Schrauben, Platten und externen Fixateuren für die Behandlung von Knochenbrüchen. 189 $^{189}$ Auch wenn die konzerninterne Konkurrenz, etwa aus China und Puerto Rico, nicht zu unterschätzen ist, behauptet Selzach innerhalb Stryker einstweilen seine Position. Die Gründe hierfür seien an dieser Stelle bloss angedeutet, da wir in Kapitel 6. vertieft auf die Erfolgsfaktoren eingehen werden. Der ehemalige Konzernchef Stephen P. Macmillan hob anlässlich eines Werkbesuchs die Kriterien "Innovation", "Qualitätsbewusstsein» und "permanente Verbesserung der Kosteneffizienz» hervor. Wörtlich: «In Selzach habe ich alle diese Eigenschaften angetroffen, weshalb wir gern in diesen Standort investiert haben.»190 Prägnant weist auch Thomas Wahl auf die Bedeutung der menschlichen Arbeitskraft hin: «Im Traumabereich hatte Osteo Erfahrung, Know-how und den entsprechenden Talentlevel. Die Leute haben Präzision im Blut.»191

Siehe Anhang 6: Beschäftigte Stryker Selzach zwischen 2007 und 2018, S. 114

\subsection{Weltmarktführer Ypsomed}

Die Ypsomed Holding AG geht auf die Disetronic AG zurück, die Willy Michel zusammen mit seinem Bruder 1984 in Burgdorf gründete. Als Aussendienst-Insulinverkäufer für Novo Nordisk hatte Michel erkannt, dass bei den Verbrauchern oft ungenau gespritzt wurde. Nach zehnjähriger Aussendiensttätigkeit entschloss er sich deshalb gemeinsam mit seinem Bruder, ein Gerät zu entwickeln, das Insulin automatisch verabreichen sollte. So entstand 1985 die erste Insulinpumpe. Da diese nicht immer zuverlässig funktionierte, wurden Insulinpens entwickelt, die bei Ausfall der Pumpe eingesetzt werden konnten. Das Pen- und Pumpengeschäft wuchs in Europa und den USA kontinuierlich. ${ }^{192}$ Gemäss einer Studie der Bank Julius Bär generierte Disetronic 1999 ihren Umsatz zu zwei Dritteln im Zusammenhang mit Diabetes-Erkrankungen. Sie hatte bei Insulinpumpen in Europa einen Marktanteil von 80 Prozent, in den USA von 20 Prozent. ${ }^{193}$

Trotz dieser eindrücklichen Zahlen stiess Disetronic im Bereich der Insulinpumpen an ihre Grenzen. So hatte etwa 2002 der Konkurrent Medtronic dreimal

\footnotetext{
189 Eigenwerbung, in: TOP 100, November 2016, S. 21.

190 Schaible, Franz: Thomas Wahl: «Selzach ist ein Vorzeigewerk», in: Solothurner Zeitung 23.6.2013.

191 Gespräch mit Thomas Wahl.

192 Gespräch mit Simon Michel.

193 Gachnang, Unternehmerportraits Medizinaltechnik, S.4-5.
} 


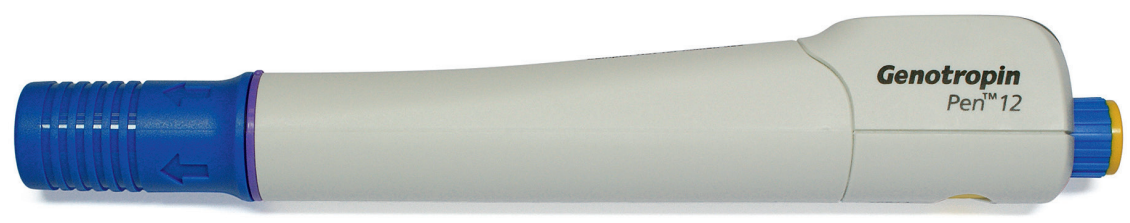

Gentropin-Spritze 1995

(C) Ypsomed AG

mehr Leute im Aussendienst als die Burgdorfer Fima. Ein Jahr später verkaufte deshalb Disetronic ihre gesamte Produktion für 1,6 Mrd. CHF an die Roche-Holding, kaufte aber das Pen-Geschäft für 500 Mio. CHF gleich wieder zurück. Diese Fokussierung war beabsichtigt, denn spätestens nach der Jahrtausendwende wurde deutlich, dass immer mehr neu entwickelte Medikamente gespritzt werden mussten, da bei oraler Verabreichung aufgrund der grossen Moleküle der Magen überlastet gewesen wäre. Nach ihrer Neuformierung nannte sich die neue, redimensionierte Firma Ypsomed Holding AG.

Dank des sprunghaft wachsenden Erfolgs des Diabetes-Medikamentes Lantus stiegen bei Ypsomed die Bestellungen des französischen Chemiekonzerns SanofiAventis rapide an. Eine verstärkte Automatisierung der Produktion wurde unumgänglich. Die drei Standorte in Burgdorf konnten die dafür erforderliche Fläche ein Automat ist 35 Meter lang - nicht mehr aufbringen. Ypsomed wurde in Solothurn fündig, weil die ebenfalls im Kunststoffspritzguss tätige Firma Flextronics die von der Ascom gekauften Hallen wieder loswerden und den dortigen Standort aufgeben wollte. Zwischen Ende 2003 und 2005 wurden schrittweise das gesamte Areal sowie fast 150 Mitarbeiter von Flextronics übernommen. Im Vordergrund standen zunächst die freiwerdenden Raumkapazitäten. Dass gleichzeitig die spezifischen Fachkräfte zur Verfügung standen, wertet der heutige CEO Simon Michel nachträglich gleichsam als «glückliche» Zugabe: «Die Kunststoffspritzgusstechnologie ist primär Erfahrung. Unsere Anlagen waren fast identisch mit jenen von Flextronics. Zudem waren die Leute bereits an Schichtarbeit gewohnt. Das war ein Glücksfall für uns, für die Arbeitnehmer und für den Kanton.» ${ }^{194}$ 


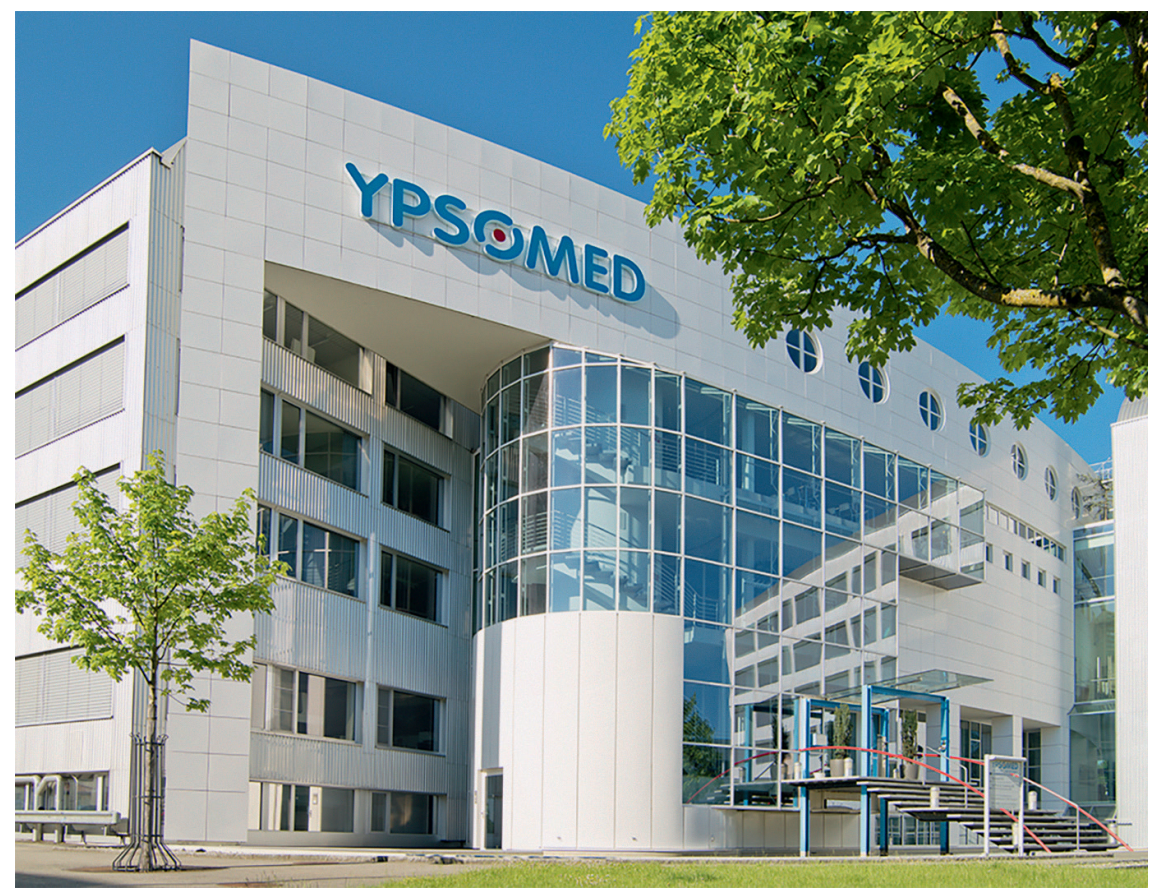

Ypsomed-Hauptgebäude

(c) Ypsomed $A G$

Die Mitarbeiterzahl am Standort Solothurn vergrösserte sich sehr rasch. Zu Beginn des Jahres 2004 waren es 15 Personen, die bis zum Frühjahr 2005 auf über 170 Beschäftigte und ein neu geschaffenes Managementteam anwuchsen. Im Frühjahr 2006 zählte Ypsomed Solothurn bereits 290, im Herbst desselben Jahres 320 Arbeitskräfte. ${ }^{195}$ Bei den Zahlen von 2006 waren allerdings die Mitarbeitenden von Ypsotec Grenchen mitgerechnet. Diese machten zu diesem Zeitpunkt ungefähr hundert Personen aus. Bereits 1994 hatte Disetronic die damalige Décolletage AG Grenchen übernommen. Es handelte sich um einen Zulieferer für die Metallverarbeitung, der sich danach an den hohen Standards der Medizintechnik orientierte. Bereits in den 1980er-Jahren hatte die Fabrik CNC-Maschinen eingeführt, die die Tätigkeit der Décolleteure und Polymechaniker grundlegend veränderten. Die Décolletage AG wurde von Disetronic gekauft, weil die Pens vor 20-25 Jahren noch viele Metallteile aufwiesen. Mit dem Verkauf des Pumpengeschäfts der Disetronic

195 Ypsomed, Geschäftsbericht 2004/05, S.4, Ypsomed, Geschäftsbericht 2005/2006, S.17 und Schaad, Peter: Steigflug bei Ypsomed hält an, in: Der Bund 16.11.2005. 
an Roche und der damit verbundenen Umbenennung der übrig gebliebenen Penfirma in Ypsomed änderte sich der Firmenname der Décolletage AG in Ypsotec AG. 2005 wurde in Tábor (Tschechische Republik) eine Zweigniederlassung mit siebzig Mitarbeitern (Stand 2006) eröffnet. Die Ypsotec generierte 6o Prozent ihres Umsatzes mit Ypsomed. Zehn Jahre später waren es 10 Prozent und heute verbleiben gerade noch 5-6 Prozent. Sie bleibt aber nach wie vor eine Tochtergesellschaft der Ypsomed AG. Diese markante Verlagerung hängt damit zusammen, dass in der Penproduktion kaum mehr mechanische Drehteile, sondern Kunststoffelemente benötigt werden. Ypsotec bewegte sich folgerichtig von der Medizintechnik hin zu verschiedenen Industrieanwendungen und zusätzlichen Aktivitäten wie Kurzdrehen, Fräsen, Laserschweissen/-gravieren, Montage und Materialentwicklung. ${ }^{196}$

Am 22. September 2004 ging Ypsomed an die Börse, wobei der Firmengründer Willy Michel 71 Prozent der Aktien behielt, 27 Prozent frei gehandelt wurden und 2 Prozent an Management und Personal gingen. ${ }^{197}$ Im Geschäftsjahr 2004/05 steigerte die Firma ihren Umsatz um 21,7 Prozent auf 241,8 Mio. CHF. Das Wachstum des gesamten Unternehmens spiegelt sich auch in der Entwicklung der Beschäftigten wider. Deren Gesamtzahl (Burgdorf und Solothurn) wuchs von $420 \mathrm{im} \mathrm{Ge-}$ schäftsjahr 2001/O2 auf 891 im Geschäftsjahr 2004/05.198 Dieser Aufschwung ist stark geprägt durch die bereits erwähnte Beziehung zu Sanofi-Aventis. Die Zusammenarbeit ging beinahe auf das Gründungsjahr von Disetronic zurück und führte zusehends zu einer gegenseitigen Abhängigkeit. Als "Hoflieferant» erzielte Ypsomed dank des Lantus-Insulins von Sanofi-Aventis enorme Gewinne. ${ }^{199} \mathrm{Ab} 2006$ begann der französische Chemiekonzern, selber sowie bei andern Lieferanten Pens zu produzieren. Der daraus entstehende Bestellrückgang führte bei Ypsomed im Geschäftsjahr 2005/o6 zu einem Abbau der temporär Beschäftigten, bei gleichzeitiger Zunahme der Festangestellten, insbesondere im Qualitätsmanagement und in der Technologie. ${ }^{200}$ Ein weiterer Personalabbau war aber nicht aufzuhalten. So mussten im Jahre 2010 erneut 66 Kündigungen ausgesprochen werden, nachdem das Aventis-Geschäft weiterhin stetig schrumpfte. Im Geschäftsjahr 2005/o6 generierte Ypsomed 64 Prozent des Umsatzes mit Sanofi-Aventis, fünf Jahre später betrug der betreffende Anteil noch 24 Prozent. ${ }^{201}$

\footnotetext{
196 Meytre, Maurice: Die 10o-jährige Erfolgsgeschichte der Décolletage Ypsotec AG, in: Ypsotec (Hrsg.), 100 Jahre Ypsotec, Kummer, Angela: 100 Jahre Industriegeschichte, in: Ypsotec (Hrsg.), 100 Jahre Ypsotec und Gespräch mit Simon Michel.

197 Schaad, Peter: 200 neue Stellen in Solothurn, in: Der Bund 14.11.2004.

198 Ypsomed, Geschäftsbericht 2004/2005, S. 32 und 55.

199 Ypsomed, Geschäftsbericht 2004/2005, S. 3.

200 Yposomed, Geschäftsbericht 2006/2007, S. 27.

201 Ypsomed, Geschäftsbericht 2010/2011, S. 56, Ypsomed, Geschäftsbericht 2012/2013, S. 19 und Sule, Adrian: Immer noch auf Durststrecke, in: Der Bund 27.5.2011.
} 
Zu Beginn des Jahres 2008 hatte das Unternehmen einen Patentstreit mit Sanofi-Aventis beigelegt und dadurch das Verhältnis zum französischen Chemieriesen immerhin normalisiert. ${ }^{202}$ Mit einer Verlagerung von Firmenkunden zu Endkunden und der Wiederaufnahme des Insulinpumpengeschäfts im Jahre 2009 schaffte es den Turnaround. Diese beiden Strategien führten zu einem spürbaren Wiederaufschwung. So wurden zwischen 2011 und 2018 zwanzig neue Tochtergesellschaften errichtet und rund 1000 Personen für die Schulung im Umgang mit Pumpen ausgebildet. Heute unterhält Ypsomed für Insulinpumpen virtuelle Callcenter in 17 Sprachen. Die Diversifizierung der Kundenbasis bis hin zum Endkunden im Pengeschäft besteht darin, dass der Lieferweg nicht mehr von B (Business) zu B (Business), sondern von B zu C (Customer) verläuft. Das bedeutet gleichzeitig, dass auf dem Endprodukt der Name «Ypsomed» steht. Von der Entwicklung zur Herstellung ist der Vorgang identisch wie vorher, bei der Distribution hingegen unterschiedlich. Weltweit ist Ypsomed in Kontakt mit über 150 Pharma- und Biotechfirmen. Für jeden dieser Kunden unterhält das Unternehmen eine Plattform, auf welcher der Bedarf antizipiert wird. Danach werden auf derselben Automationsschiene ähnliche, wenn auch leicht verschiedene Produkte hergestellt. Von der Plattform zum Produkt dauert es nicht mehr vier Jahre, sondern vier Monate. Simon Michel bilanziert: «Heute stellen wir für Pharmafirmen das ganze Produkt her. Die Ebit-Marge beträgt 35 Prozent. Im Entwickeln und Herstellen von Pens sind wir Weltmarktführer. Acht bis neun von zehn Deals werden über uns abgeschlossen. Wir sind praktisch Monopolist.» ${ }^{203}$ Zurzeit wächst das Pumpengeschäft um 20 Prozent, das Pengeschäft um über 10 Prozent. Zusätzlich stellt Ypsomed Blutzuckermessgeräte her. Der Aufschwung zeigt sich auch in den Eckdaten. ${ }^{204}$ Im Geschäftsjahr 2017/18 stieg der Umsatz gegenüber dem Vorjahr um 19,7 Prozent auf 466 Mio. CHF. Das Betriebsergebnis belief sich auf 61,1 Mio. CHF, der Reingewinn wurde um 12,6 Prozent von 46,2 Mio. CHF auf 52,1 Mio. CHF gesteigert. ${ }^{205}$

Nachdem der Vertrag mit dem US-Partner Insulet (Pumpenhersteller für Insulinverabreichung) im Juni 2018 auslief, will sich Ypsomed noch stärker auf eigene Produkte abstützen. Simon Michel schätzte Ende Mai 2018, dass die Produktion von Injektionssystemen für Insulin und andere Medikamente in den kommenden drei Jahren um je 25 Prozent zunehmen werde. Generell sind gemäss Michel die wirtschaftlichen Aussichten gut, da Diabetes-Erkrankungen weltweit zunehmen. ${ }^{206}$

\footnotetext{
202 Müller, Giorgio V.: Ypsomed auf dem Weg zur Emanzipation, in: Neue Zürcher Zeitung 28.5.2008.

203 Gespräch mit Simon Michel.

204 Ebd.

205 Ypsomed, Geschäftsbericht 2017/2018, S. 42-43.

206 Feldges, Dominik: Ypsomed muss stärker auf eigenen Füssen stehen, in: Neue Zürcher Zeitung 24.5 .2018
} 


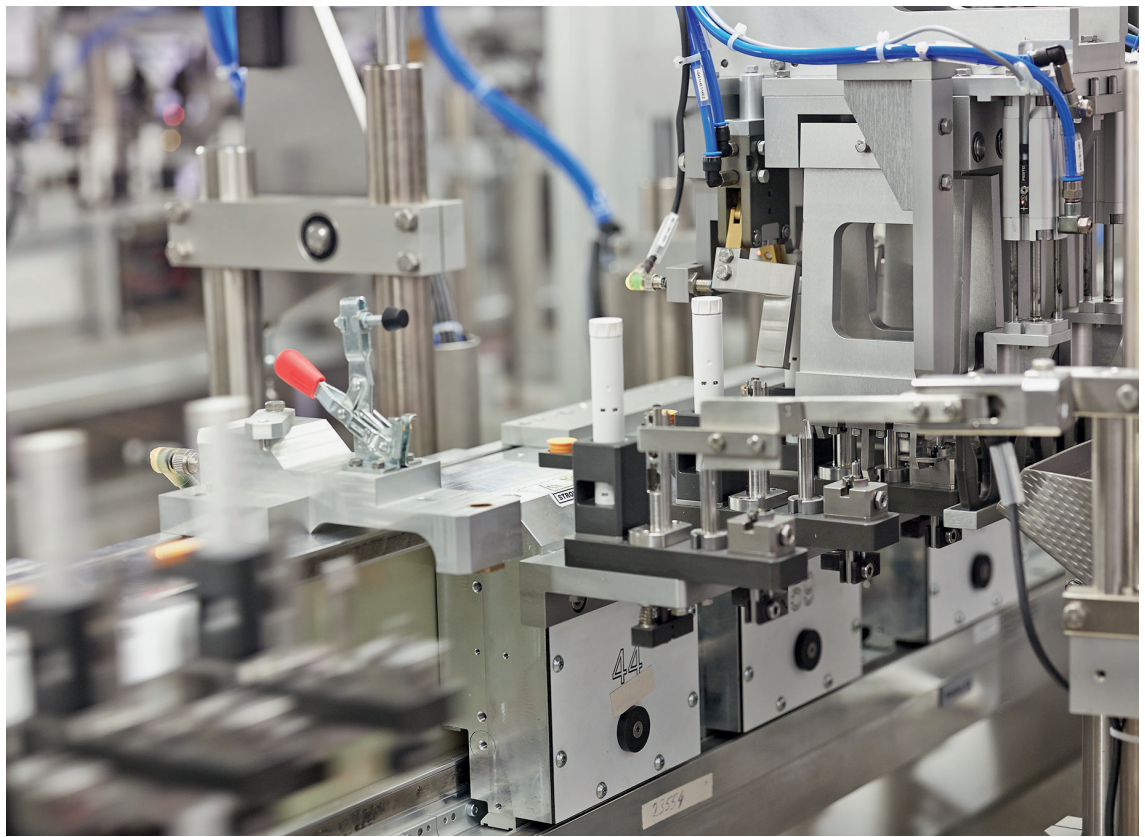

Uno-Spritzen-Produktion 2007

(c) Ypsomed $A G$

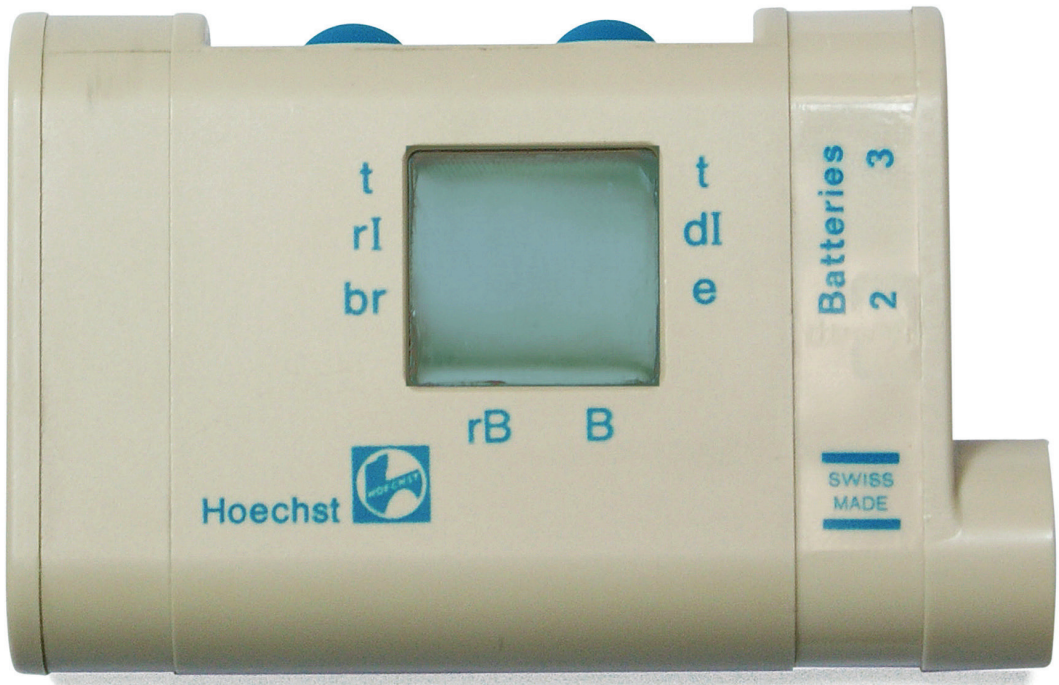

Insulinpumpe

(c) Ypsomed AG 
Die Zukunft von Ypsomed in der Schweiz macht Michel auch vom Steuerumfeld abhängig. Je nach dessen Weiterentwicklung zieht er einen Umzug des Hauptsitzes von Burgdorf nach Solothurn in Betracht. ${ }^{207}$ Seit jeher ist Ypsomed gegenüber Produktionsverlagerungen in Billiglohnländer restriktiv. Im Wissen um die Qualität der Arbeitskräfte geniesst die Schweiz als Produktionsstandort Priorität. Diese Vorgehensweise bedingt technologische Vorsprünge gegenüber der Konkurrenz, ein ausgeprägtes Mass an Automatisierung und demzufolge auch hohe Forschungsinvestitionen. ${ }^{208}$ So wurden 20168 Prozent der Kosten für Qualifizierung ausgegeben. Zudem arbeiten rund hundert Personen aus Deutschland in Solothurn und Burgdorf, weil es bestimmte Ausbildungen in der Schweiz nicht gibt. Produkte mit hohem manuellem Aufwand werden allerdings schon seit einiger Zeit in billiger produzierende Staaten verlagert. «Wenn die Herstellung eines Produktes mehr als zwei Minuten dauert, macht die Produktion in der Schweiz keinen Sinn mehr», erklärte Simon Michel anlässlich eines Podiumsgesprächs. ${ }^{209}$ Auch wenn die Beschäftigtenzahlen im Ausland verhältnismässig stärker wachsen und im Frühjahr 2019 in Schwerin (D) eine Fabrik mit 200 Mitarbeitern eröffnet werden soll, arbeiten für Ypsomed immer noch deutlich mehr Personen in der Schweiz als im Ausland (2018: 1026 gegenüber 471). ${ }^{210}$

Siehe Anhang 7: Beschäftigte Ypsomed Holding AG zwischen 2005 und 2018, S. 115

Siehe Anhang 8: Beschäftigte Ypsomed Holding AG an den Standorten Burgdorf und Solothurn 2012-2018, S. 116

207 Gespräch mit Simon Michel.

208 Ypsomed, Geschäftsbericht 2004/2005, S.3-4.

209 Fluri, Lucien: Weniger als zwei Minuten sollte die Produktion in der Schweiz dauern, in: Solothurner Zeitung 18.8.2016.

210 Gespräch mit Simon Michel. 


\section{Rahmenbedingungen und Erfolgsfaktoren}

Der Jurasüdfuss ist im Laufe der Jahrzehnte zu einem weltweiten Hotspot der Medizintechnik geworden. Auf diesem Weg hat er andere, konkurrierende Regionen ausgeschaltet. Es drängt sich deshalb die Frage auf, welche Rahmenbedingungen und Faktoren diesen Erfolg ermöglicht haben. Und, vertieft nachgefragt, wieweit diese allgemeiner Art und wieweit diese spezifisch sind.

Im gleichen Atemzug wie die Medizintechnik wird oft die Uhrenindustrie genannt. Tatsächlich sind die Überschneidungen mannigfach. Sie betreffen die Qualifikationen, die Berufsfunktionen und die Mentalität der Mitarbeitenden, die Betriebsstruktur, in der KMU eine wichtige Rolle einnehmen, sowie gemeinsame regionale Schwerpunkte, zu denen auch der Jurasüdfuss gehört.

Die Frage nach dem gesamtindustriellen und sozioökonomischen Umfeld für die Entstehung und Entwicklung der regionalen Medizintechnik stellt sich über deren Verbindung mit der Uhrenindustrie hinaus. Von besonderem Interesse sind die spezifischen Merkmale der Industrialisierung und der Stellenwert des sekundären Sektors im Raum Grenchen-Solothurn. Im Vordergrund steht dabei der Einfluss der entsprechenden Faktoren auf die Geschichte der Medizintechnik. Vor diesem Hintergrund spielen die Verschiebungen in den 1970er-Jahren weg von der Uhren- und Maschinenindustrie hin zu anderen, teils neu entstandenen Sektoren eine nicht zu unterschätzende Rolle.

Neben der Uhrenindustrie und der spezifischen Industriestruktur schälen sich weitere Erfolgsursachen heraus, die - etwas allgemein gesagt - allesamt auf die Bedeutung der menschlichen Arbeitskraft hindeuten. Auffallend ist dabei die Heterogenität der beteiligten Akteure. Die Rede ist von Chirurgen, Technikern, Ingenieuren, Managern, Facharbeitern. Die blosse Aufzählung dieser Personengruppen erklärt den Aufbau der neuen Branche noch unzureichend. Schon wesentlich weiter führt eine genaue Beschreibung von deren Wertvorstellungen, Einstellungen, Verhaltensweisen. Diese sind teilweise spezifisch und formen sich im Zusammenwirken der verschiedenen Beteiligten zu einer Art Alleinstellungsmerkmal. 


\subsection{Fruchtbarer Boden dank Uhrenindustrie}

Die Bedeutung der Uhrenindustrie für die Entstehung der Medizintechnik wird deutlich, wenn man die Geschichte dieser beiden Branchen vergleicht. Nachdem in den Kapiteln 4 und 5 die Entstehung und Entwicklung der Medizintechnik in der Schweiz und am Jurasüdfuss ausführlich dargestellt worden ist, liefern wir zunächst einen historischen Abriss der schweizerischen und regionalen Uhrenindustrie. Danach richtet sich das Augenmerk auf Gemeinsamkeiten und Unterschiede der beiden Wirtschaftszweige.

Die Ursprünge der schweizerischen Uhrenindustrie gehen auf die Schmuckherstellung und das Darstellen religiöser Motive durch Genfer Goldschmiede zurück. Nachdem Calvin das Schmucktragen und religiöse Abbildungen verboten hatte, wandten sich die betroffenen Handwerker der Uhrenproduktion zu. ${ }^{211}$ Die meisten unter ihnen arbeiteten in Kleinstbetrieben oder in Heimarbeit. Die stetig steigende Nachfrage führte zu immer stärkerer Arbeitsteilung mit bis zu hundert verschiedenen Arbeitsgängen, was der Tendenz zu einer betriebsökonomischen Mikrostruktur noch Vorschub leistete. Das Produktionswachstum sprengte gleichzeitig die Genfer Zunftschranken, die Uhrenherstellung dehnte sich auf das Vallée de Joux, den Neuenburger Jura und das Tal von St. Imier aus. So wuchs die Zahl der Uhrmacher im Kanton Neuenburg von 464 (1752) auf 3634 (1788). ${ }^{212}$ Früh entstanden in der Uhrenindustrie regionale Schwerpunkte («districts industriels»), die eine ausgeprägte Spezialisierung und Vielfalt der Produkte sowie rasche Anpassungen der Produktionsweise ermöglichten: «Les districts industriels permettent ainsi la fabrication de produits spécialisés de manière flexible et compétitive face à la production de masse standardisée des grandes entreprises.» ${ }^{213}$

Mitte des 19. Jahrhunderts hielt die Uhrenindustrie Einzug im Kanton Solothurn. 1856 gründeten der Schullehrer Urs Schild und der Arzt Joseph Girard in Grenchen eine Fabrik für Uhrwerke (Ebauches). Girard hatte dabei ein sozialpolitisches Motiv, wollte er doch die Lebenssituation im mausarmen Ort am Jurasüdfuss verbessern. Die Instruktion der lokal entstehenden Facharbeiterschaft übernahmen Spezialisten aus der Westschweiz. Zwischen 1862 und 1871 entstanden in Grenchen weitere Fabriken. Der erste von Schild und Girard errichtete Betrieb dehnte ab 1876 die Produktion auf die Herstellung ganzer Uhren aus. In der Stadt Solothurn gehen die Anfänge der Uhrenindustrie auf das Jahr 1860 zurück. Dreizehn Jahre später erfolgte die Gründung der Ebauches-Fabrik Lanco in Langendorf. Diese Firma war 1890 mit

211 Donzé, Histoire de l'industrie horlogère suisse, S.12-14.

212 Bergier, Die Wirtschaftsgeschichte der Schweiz, S. 182 und Donzé, Histoire de l'industrie horlogère su-

isse, S.14-16.

213 Ebd., S. 4O. 
rund 1000 Beschäftigten das weltgrösste Uhrenunternehmen. ${ }^{214}$ Diese Zahl deutet auf den generellen Aufschwung in der Region hin, wie Pierre Yves Donzé festhält: "Quant à Granges, qui voit le nombre de ses habitants passer de 1600 en 1850 à 5200 en 1900, elle acceuille notamment des fabriques d'ébauches, les sociétés Eterna (1856), A. Schild SA (1896) et A. Michel SA (1898), entreprises qui forment le coeur du trust Ebauches SA dans l'entre-deux-guerres.»15 Mit der Gründung der Firma Thommen in Waldenburg 1870 verbreitete sich die Uhrenindustrie auch jenseits des Jura. Diese Erweiterung ist für die Entwicklung der Medizintechnik insofern von Bedeutung, als dadurch der Grundstein für die Firma Straumann (später aufgegangen in Synthes) gelegt wurde.

Das Wachstum der Solothurner Uhrenindustrie war nunmehr Teil einer gesamtschweizerischen Entwicklung. Die erwähnte Mechanisierung erlaubte eine Massenproduktion. Um die Jahrhundertwende hatte die schweizerische Uhrenindustrie wieder einen Weltmarktanteil von 90 Prozent. ${ }^{216}$ Nach einem vorübergehenden Rückgang brachte der Erste Weltkrieg mit seiner sprunghaft steigenden Nachfrage nach Armbanduhren für Soldaten erneut eine rasante Steigerung des Exportes mit sich. In der Zwischenkriegszeit war die Uhrenindustrie wichtigster Devisenbringer für die Schweiz. Gleichzeitig wuchs der Graben zwischen der modernen Ebauches- und Bestandteileproduktion und der handwerklich geprägten Uhrenmontage. Infolge von Überproduktionen bei Ersterer wurden zusehends mehr Uhrwerke ins Ausland exportiert. Durch diese als «Schablonieren» bezeichnete Praxis drohte eine Auslandsverlagerung der gesamten Schweizer Uhrenindustrie. ${ }^{217}$ Zur besseren Kontrolle der Uhrwerkexporte gründeten die Schild AG Grenchen, die Fabrique d'Horlogerie Fontainemelon und die Ad. Michel AG Grenchen 1926 die Ebauches Holding SA Neuenburg. ${ }^{218}$ Mit gleicher Absicht fusionierten die Firmen Omega und Tissot 1930 zur Société Suisse pour l'Industrie Horlogère (SSIH). Der ganz grosse Zusammenschluss erfolgte jedoch 1931 mit der von Banken und Bund unterstützten Superholding Allgemeine Schweizerische Uhrenindustrie AG (ASUAG), die 1934 durch den Bundesbeschluss zum Schutze der Uhrenindustrie (Uhrenstatut) vom öffentlich-rechtlichen zum gesetzlichen Kartell wurde. ${ }^{219}$ Dieses sah für Neugründungen und Erweiterungen von Uhrenfabriken und für den Export eine rigide Bewilligungspflicht vor. ${ }^{220}$ Anstelle einer Vertikalisierung wur-

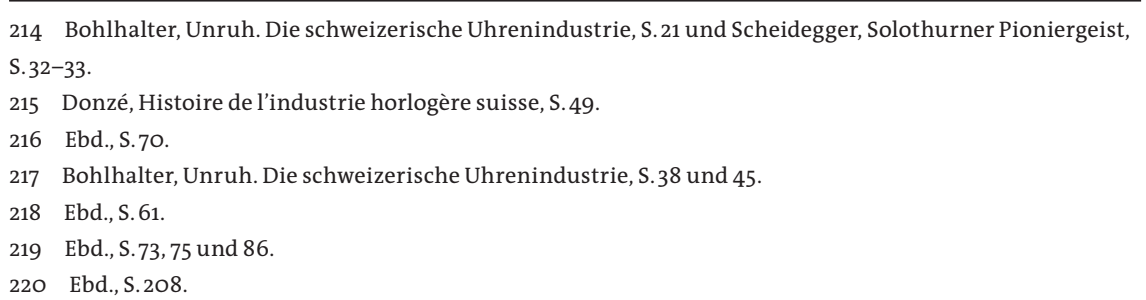


den - auch aus staatspolitischen Gründen - in der Zusammensetzung von Uhren kleinbetriebliche Strukturen mit extremer Arbeitsteilung aufrechterhalten. ${ }^{221}$ Die ausgeprägte Dezentralisierung traf auch für den Kanton Solothurn zu. Gleichzeitig fand im Raum Grenchen/Lebern eine starke Konzentration statt. ${ }^{222}$ Der Wirtschaftshistoriker Wolfgang Hafner bezeichnet diese Epoche als «goldene Zeiten der Uhrenindustrie». ${ }^{223}$

Die Kehrseite von Kartell und Protektionismus war ein sukzessiver Verlust der internationalen Konkurrenzfähigkeit. Fachleute sind sich darin einig, dass die Krise der Uhrenindustrie zwischen 1970 und 1976, in der 35000 von total 90000 Arbeitsplätzen verlorengingen, nicht eine Technologie-, sondern eine Strukturkrise war. ${ }^{224}$ Stellvertretend für andere, hält Pierre Yves Donzé fest: «Le principal problème de l'industrie horlogère suisse des années 1970 est qu'elle n'est pas compétitive sur le marché mondial [...] les montres suisses sont trop chères, notamment en raison d'un appareil de production non rationalisé ... $\rangle^{225}$ Nicht zufällig fiel 1970 das Uhrenstatut. Es darf allerdings nicht übersehen werden, dass die Uhrenkrise in den grössten Wirtschaftseinbruch der Schweiz seit den 1930er-Jahren integriert war, mit einem Negativwachstum von 7 Prozent im Jahre 1975 und dem Verlust von rund 300000 Arbeitsplätzen. ${ }^{226}$ Die zweite, durch den Technologiewandel von der mechanischen zur elektronischen Uhr bedingte Krise forderte zwischen 1976 und 1984 nochmals 25000 Arbeitsstellen. ${ }^{227}$

Die Wende erfolgte 1983 mit der Erfindung der Kunststoffuhr Swatch, die Elektronik mit einer Analoganzeige kombiniert und wesentlich weniger Teile als eine konventionelle Uhr benötigt. ${ }^{228}$ Dieser technologische Sprung fand auch darin seinen Ausdruck, dass 1985 die zwei Jahre zuvor fusionierten SSIH und ASUAG ihre Firmenbezeichnung in "Société Suisse de Microéléctronique et d'Horlogerie SA» (SMH) umwandelten. ${ }^{229}$ Diese wurde 1998 in Swatch Group umbenannt - ein Zusammenschluss, der alle Preiskategorien vereinigte und eine vertikale Konzentration vornahm. ${ }^{230}$ Die Swatch Group dominiert heute die schweizerische Uhrenpro-

\footnotetext{
221 Ebd., S.101.

222 Hafner, Die Krise und ihre Bewältigung, S. 51-53.

223 Ebd., S.33.

224 Bohlhalter, Unruh. Die schweizerische Uhrenindustrie, S. 120-121, 224 und 255.

225 Donzé, Histoire de l'industrie horlogère suisse, S.161.

226 Lipp, Standort Schweiz im Umbruch, S. 24.

227 Bohlhalter, Unruh. Die schweizerische Uhrenindustrie, S. 239-240.

228 Trueb, Lucien F.: Die Quarzrevolution. Von der Mechanik zur Elektronik und zurück, in: Betschon/Betschon/Lindecker/Schlachter, Ingenieure bauen die Schweiz, S. 372.

229 Bohlhalter, Unruh. Die schweizerische Uhrenindustrie, S. 276.

230 Donzé, Histoire de l'industrie horlogère suisse, S. 165 und 171.
} 
duktion. Sie zählt mehr als 36 ooo Mitarbeitende in rund fünfzig Ländern und konzentriert Forschung und Entwicklung in der ETA Grenchen. ${ }^{231}$

Die Querverbindung zwischen Uhrenindustrie und Medizintechnik bringt ein Titel des Tages-Anzeigers vom 25. Mai 2018 auf den Punkt: «Medtech-Firmen profitieren von der Uhrenbranche». Und die Zeitung präzisiert im Untertitel: «Ohne Uhrenindustrie gäbe es weder Herzschrittmacher noch chirurgische Instrumente». ${ }^{232}$ Seit Jahrzehnten ist es tatsächlich unbestritten, dass die Uhrenindustrie bei der Entstehung der Medizintechnik in der Schweiz eine zentrale Rolle wahrgenommen hat. Noch etwas spezifischer wird in der Zeitschrift Cash darauf hingewiesen, die Branche verbinde «die hohe Fertigungsqualität und die Präzision der Schweizer Uhrmachertradition mit dem medizinischen Know-how der heutigen Pharma-Unternehmen». ${ }^{233}$ Sinn für Genauigkeit, Konzentrationsfähigkeit, ein hohes Mass an Feinmotorik, Sauberkeit sind zweifellos Eigenschaften, die in beiden Sektoren bedeutend sind. So ist es nicht verwunderlich, dass der massive Arbeitsplatzverlust in der schweizerischen Uhrenindustrie zwischen 1970 und 1986 eine spürbare Verschiebung hin zur Medizintechnik ausgelöst hat. Bruno Bohlhalter weist in seinem historischen Rückblick zur Uhrenindustrie explizit auf diesen Umstand hin: «Aufgrund ihrer spezifischen Kenntnisse und feinmotorischen Fähigkeiten suchten viele dieser Fachleute ihre Chancen in verwandten Gebieten wahr zu nehmen, wie zum Beispiel in der Medizinaltechnik oder dem Apparateund Maschinenbau.»234 Diese Migrationsbewegung wurde auch von anderen fachkundigen Beobachtern dargestellt: «Die Uhrenindustrie entliess mit ihrem Niedergang Personal auf den Arbeitsmarkt, dem der Umgang mit Tausendstelmillimetern - Dimensionen, die in der Medizintechnik gang und gäbe sind - keine Mühe bereitete. $\aleph^{235}$

Von allen Seiten und unter verschiedenen Aspekten werden die Gemeinsamkeiten hervorgehoben. Bei so viel Einstimmigkeit stellt sich die Frage, wie weit diese reichen und ob es Unterschiede gibt. Auf die Verwandtschaft der Branchen weist auch Robert Mathys jun. hin, wenn er betont, eine mikrogenaue Fertigung und die Art der Oberflächenbehandlung seien beiden gemeinsam. Er legt den Akzent aber auch auf Unterschiede. Die Uhrenindustrie verwende das viel leichter bearbeitbare Messing, während die Medizintechnik mit harten, zähen Materialien wie rostfreiem Stahl oder Titan zurechtkommen müsse. Im Gegensatz zur Uhrenindustrie, wo der 1/100o mm gelte, genüge in seinem Umfeld der 1/10 mm. Wörtlich: «Die

231 Swatch Group, Geschäftsbericht 2018, S. 5 und 12.

232 Mettler, Jon: Medtech-Firmen profitieren von der Uhrenbranche, in: Tages-Anzeiger 21.5.2018.

233 Schaffner, Andreas: Ersatzteile für müde Knochen, in: Cash 15.7.2004.

234 Bohlhalter, Unruh. Die schweizerische Uhrenindustrie, S. 243.

235 Städeli, Markus: Medtech-Eldorado Schweiz, in: Handelszeitung 8.11.200o. 
Materialien in der Uhrenindustrie und bei uns sind ganz verschieden, hingegen ist die Vorstellung von Décolletage gleich. In der Uhrenindustrie ist alles filigraner, die Technologie an sich (Fräsen, Bohren) ist dieselbe. Unterschiedlich sind die Grössenordnungen, die Materialien und die Werkzeuge, die man anwendet.»136 Eher noch deutlicher äussert sich Robert Frigg: «Uhrenindustrie und Medtech sind zwei Paar Schuhe. Bezüglich des Materials und der Präzision gibt es Unterschiede. Gemeinsam ist das Qualitätsbewusstsein in beiden Branchen. ${ }^{237}$ Es ist aufschlussreich, wie die professionelle Wahrnehmung der beiden Fachleute die landläufige Meinung relativiert.

Dessen ungeachtet, ist die Nähe zwischen Uhrenindustrie und Medizintechnik unübersehbar. Sie äussert sich nicht zuletzt in der Bildung von mehreren regionalen Schwerpunkten (z. B. Jurasüdfuss, St. Imier, Le Locle, Region zwischen Lausanne und Genf), wo die Beschäftigten über die erforderlichen Kenntnisse verfügen. ${ }^{238} \mathrm{Lu}$ kas Eschbach, stellvertretender Geschäftsleiter der RMS Foundation, präzisiert diesen Sachverhalt: «Viele Medtech-Unternehmen hier in der Region haben eine Tradition, die aus der Uhrenindustrie herausgekommen ist. ${ }^{239}$ Vergleichbar sind im Weiteren auch die betrieblichen Strukturen. Neben wenigen, allerdings den Markt dominierenden Grossfirmen nehmen KMU eine wichtige Stellung ein. Bereits Mitte des 20. Jahrhunderts stiegen mehrere Zulieferer von Uhrenfirmen in die Medizintechnik ein. Eine zweite Welle von Neuorientierung fand im Anschluss an die Quarzkrise statt. So stellte etwa Tissot dank der Expertise von Uhrenfachleuten in Zusammenarbeit mit Roche Bestandteile für Herzschrittmacher her. Sehr viele Betriebe beliefern heute Uhrenindustrie und Medizintechnik gleichzeitig. ${ }^{240}$

Historische Unterschiede zeigen sich zwischen den beiden Wirtschaftszweigen bei der staatlichen Politik. Während die Uhrenindustrie zwischen 1934 und 1970 mit dem Uhrenstatut eine vom Bund abgesicherte Protektion genoss, bewegte sich die Medizintechnik stets auf dem freien Markt. Es sei denn, man betrachtet die Monopolstellung von Mathys AG, Straumann und Synthes USA bezüglich der Synthes-Produkte als vergleichbares Kartell. Diese Privilegierung war jedoch nicht beschäftigungs- und staatspolitisch motiviert, sondern diente der AO zur Qualitätssicherung. Im Gegensatz zur Uhrenindustrie ist die Medizintechnik auch kein in sich geschlossener Produktionszweig; sie hat vielmehr Querschnittcharakter (siehe S.12).

\footnotetext{
236 Gespräch mit Robert Mathys jun.

237 Gespräch mit Robert Frigg.

238 Dümmler, Wissensbasierte Cluster in der Schweiz, S.94.

239 Eschbach, Medtech im Kanton Solothurn.

240 Breiding/Schwarz, Wirtschaftswunder Schweiz, S. 91 und Mettler, Jon: Medtech-Firmen profitieren von der Uhrenbranche, in: Tages-Anzeiger 21.5.2018.
} 
Fazit: Wenn auch gewisse Abweichungen zwischen Uhrenindustrie und Medizintechnik festzumachen sind, überwiegt eindeutig das Gemeinsame, vor allem, was die Qualifikations- und Betriebsstruktur sowie die regionale Schwerpunktbildung anbetrifft.

\subsection{Industriell geprägte Region}

Die Industrialisierung setzte am Jurasüdfuss vergleichsweise spät, dann allerdings sehr rasant ein. Als Beispiele seien die von Roll AG (gegründet 1823, ab 1873 mit Sitz in Gerlafingen) und die Kammgarnfabrik Derendingen (gegründet 1872) erwähnt. Die ersten Fabrikarbeiter waren überwiegend Einwanderer aus ländlichen Regionen. ${ }^{241}$ Später, mit der Gründung der Scintilla durch die BBC (1917), setzten neue Industriezweige bereits an den Fähigkeiten der Uhrenarbeiter an. Urs Ruepp, 19832005 Leiter Entwicklung der Scintilla, unterstreicht diesen Wandel: «Solothurn wurde als Standort gewählt, weil sich hier nach Ansicht der Unternehmensgründer eine Arbeiterschaft befand, die dank der Uhrenindustrie fähig war, präzis zu

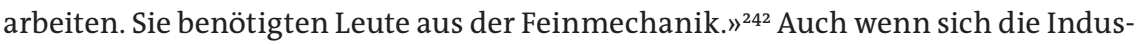
triestruktur im Kanton Solothurn sukzessive verschob, blieb die Metall- und Maschinenindustrie ein Leitsektor, in dem noch zu Beginn der 1980er-Jahre der Beschäftigungsanteil innerhalb des Sekundärsektors 55 Prozent betrug. Zusammen mit der Uhrenindustrie blieb deshalb die Solothurner Wirtschaft stets stark der internationalen Konkurrenz ausgesetzt. ${ }^{243}$ Diese Tatsache hängt nicht nur mit der Art der dominierenden Branchen, sondern generell mit der Industrielastigkeit des Kantons zusammen. So schreibt die Crédit Suisse in ihrer volkswirtschaftlichen Einschätzung über den Jurasüdfuss: «Der Wirtschaftsraum Jurasüdfuss ist industriell geprägt. Über die Jahre hat sich der Fokus von der Schwerindustrie mehr und mehr auf hochtechnologische Produkte mit extrem hohem Innovationsgrad verschoben. Die bedeutendste Branche ist heute die Herstellung von Elektronik und Uhren, die auch die Herstellung von Präzisionsinstrumenten und Optik umfasst ..." ${ }^{244}$ Auch wenn die Crédit Suisse den "Jurasüdfuss» (berechtigterweise) geografisch breiter fasst, als dies in unserer Studie der Fall ist, gilt ihre Aussage in hohem

241 Kienzle, «Es gibt nur ein Gerlafingen!», S. 254.

242 Ruepp, Urs, zit. nach: Hafner, Die Krise und ihre Bewältigung, S.143.

243 Frick/Holenstein, Innovationskraft und Innovationsverhalten, S.1.

244 Crédit Suisse (Hrsg.), Regionalstudie Wirtschaftsraum Jurasüdfuss, S.18. 
Masse gerade auch für die Region Grenchen-Solothurn, wie die Aufteilung der Beschäftigten nach Wirtschaftssektoren für das Jahr 2012 aufzeigt ${ }^{245}$ :

\begin{tabular}{|l|r|r|r|}
\hline Wirtschaftsregion & Beschäftigte Sektor 1 & Beschäftigte Sektor 2 & Beschäftigte Sektor 3 \\
\hline Grenchen & $482(3,1 \%)$ & $9592(61,3 \%)$ & $5557(35,5 \%)$ \\
\hline Solothurn & $895(2,4 \%)$ & $11394(30,7 \%)$ & $24770(60,8 \%)$ \\
\hline
\end{tabular}

Bezogen auf den ganzen Kanton, war 2016 rund ein Drittel der Arbeitnehmer in produzierenden Betrieben tätig. Das waren fast 10 Prozent mehr als im schweizerischen Durchschnitt. ${ }^{246}$

Die markanteste Verschiebung innerhalb der solothurnischen Industrie erfolgte im Anschluss an den Einbruch Mitte der 1970er-Jahre, nachdem die kantonale Wirtschaft im Jahrzehnt zuvor noch eine eigentliche Hochblüte erlebt hatte. Neben den beiden Uhrenkrisen zwischen 1970 und 1984 erschütterten auch andere massive Produktionseinbussen, etwa bei von Roll und Bally, die ökonomische Struktur bis hinein in die 1990er-Jahre. Stellenabbau, Teil- und Totalschliessungen waren an der Tagesordnung. So schnellte die Arbeitslosenzahl von 1644 im Jahre 1993 auf 5892 im folgenden Jahr hoch. ${ }^{247}$

Diese Erschütterungen waren begleitet von neuen technischen Entwicklungen, die bei verschiedenen Wirtschaftsakteuren neue Energien freisetzten, wie Wolfgang Hafner festhält: "Offenbar haben es einzelne Unternehmen der solothurnischen Industrielandschaft geschafft, diesen Wandel in der Produktion rechtzeitig vorzunehmen und sich entsprechend zu fokussieren. Anderen wiederum gelang es, sich aufgrund spezifischer Qualifikationen der Arbeitskraft - wie etwa bei der Medizintechnik - eine Nische zu sichern und sich da zu etablieren. ${ }^{248}$ Es fällt auf, dass - gleichsam als Antwort auf die Forderung nach einer verstärkten Tertiarisierung weiterhin ein beachtlicher Anteil von Produkten mit einer hohen Wertschöpfung hergestellt werden konnte. ${ }^{249}$ Von Vorteil waren dabei sicher die Überschaubarkeit dank relativ kleiner Einheiten und die Stabilität bestehender Netzwerke. ${ }^{250}$

Diese Erfolge dürfen aber nicht darüber hinwegtäuschen, dass viele «Fehlentwicklungen bei Unternehmen im Übergang von der kartellistisch-korporati-

\footnotetext{
245 Ebd., S.12.

246 Graber, Roger: Vom Uhrenhersteller zum Schweizer Hotspot für Medizintechnik, in: Handelskammerjournal 7.6.2016.

247 Wyser, Alfred: Der Kanton Solothurn im 20. Jahrhundert - Versuch einer historischen Übersicht, in: Jahrbuch für Solothurnische Geschichte 73 (2000), S. 97-98.

248 Hafner, Die Krise und ihre Bewältigung, S. 21.

249 Ebd., S.19-20 und 37.

250 Gugerli, David/Tanner, Jakob: Wissen und Technologie, in: Halbeisen/Müller/Veyrassat, Wirtschaftsgeschichte der Schweiz, S.309.
} 
ven Wirtschaftsform in der Nachkriegszeit bis in die 1970er Jahre zur Marktwirtschaft $»^{251}$ stattgefunden haben. Nicht nur die Uhrenindustrie, sondern auch die Metall- und Maschinenindustrie oder das Fernmeldewesen (Autophon Solothurn) waren durch Protektion abgeschirmt. Der Arbeitsplatzverlust in der Industrie konnte durch eine Zunahme im Dienstleistungssektor nur teilweise aufgefangen werden. Solothurn mutierte mehr und mehr zu einem Wohnkanton, "zur Agglomeration der grossen Wirtschaftszentren $»^{252}$. Diese Entwicklung schlug sich unter anderem in der Beschäftigungsquote nieder.

\section{Beschäftigungsquote ${ }^{253}$}

\begin{tabular}{|l|l|l|l|l|l|}
\hline Jahr & 1950 & 1975 & 1985 & 1995 & 2001 \\
\hline Schweiz & $48,9 \%$ & $42,9 \%$ & $55,5 \%$ & $53,7 \%$ & $53,6 \%$ \\
\hline Solothurn & $49,9 \%$ & $42,9 \%$ & $50,9 \%$ & $48,3 \%$ & $46,8 \%$ \\
\hline
\end{tabular}

Eine ähnliche Verlaufskurve zeigt sich in der Veränderung des Volkseinkommens.

$\mathrm{VE}=$ Volkseinkommen je Einwohner $(\mathrm{CH}=100)^{254}$

\begin{tabular}{|l|l|l|l|l|l|l|l|}
\hline Jahr & 1950 & 1960 & 1970 & 1985 & 1995 & 2000 & 2005 \\
\hline Solothurn & 103 & 115 & 94 & 88 & 93 & 84 & 84 \\
\hline
\end{tabular}

Die Crédit Suisse bestätigt diese Zahlen auch für die jüngste Zeit, wenn sie in ihrer Studie über den Jurasüdfuss betont, dass Solothurn und Bern einkommensmässig unter dem Landesdurchschnitt liegen und bei privaten Einkommen relativ hohe Steuern aufweisen. ${ }^{255}$ Zusammenfassend lässt sich festhalten, dass durch die strukturellen Veränderungen seit den 1970er-Jahren ein gut qualifiziertes Arbeitskräftepotenzial für die Medizintechnik freigesetzt wurde. Dieses konnte indessen nur partiell übernommen werden. Wolfgang Hafner spricht denn auch ausdrücklich von «Nischen». Eine grössere Verschiebung oder gar eine Kompensation des Arbeitskräfteverlustes fand nicht statt.

Ungeachtet der sektoriellen Verschiebungen innerhalb der Solothurner Wirtschaft blieben die Beziehungen zwischen Arbeitgebern und Arbeitnehmern bis in die jüngste Zeit konstant. Diese Stabilität lässt sich mit der dezentralisierten Form

\footnotetext{
251 Hafner, Die Krise und ihre Bewältigung, S.195.

252 Ebd., S, 20.

253 Hiestand, Manuel/Müller, Margrit/Woitek, Ulrich: Wohlstandsverteilung und regionale Entwicklung. Partizipation der Kantone und Regionen, in: Halbeisen/Müller/Veyrassat, Wirtschaftsgeschichte der Schweiz, S. 818.

254 Ebd., S.819.

255 Crédit Suisse (Hrsg.), Regionalstudie Wirtschaftsraum Jurasüdfuss, S.12.
} 
der Industrialisierung und der damit verbundenen betrieblichen Sozialisation der Arbeitnehmer erklären. Präzis beschreibt Hafner diese Vorgänge: «Gleichzeitig fand in dieser breiten Durchdringung der Landwirtschaft durch mittlere und kleinere Uhrenateliers eine eigentliche Ausbildungswelle grosser Bevölkerungsschichten in einem feinmechanisch und feinmotorisch höchst anspruchsvollen Bereich statt, der begleitet wurde von einer Disziplinierung und Anpassung der Arbeitnehmerschaft an die Bedürfnisse der Industrie. ${ }^{256}$ Auch wenn gegen Ende des 19. Jahrhunderts die Arbeits- und Lebensbedingungen schlechter waren als in anderen vergleichbaren Industrieregionen, wurde das zaghaft entstehende Klassenbewusstsein der Arbeiter durch ein betriebliches Gemeinschaftsgefühl überlagert. Arbeitsethos, Pflichtbewusstsein und eine ausgeprägte Identifikation mit dem Betrieb charakterisierten den idealtypischen Arbeiter im Kanton Solothurn, insbesondere auch am Jurasüdfuss. ${ }^{257}$

Generell war (und ist) das soziokulturelle Klima konservativ ausgerichtet. Darauf weist auch ein oft zitierter Text im Solothurner Lied hin: «Es isch immer eso gsi». Das Bestreben, Erreichtes zu verteidigen, macht veränderungsresistent. Dies zeigt sich deutlich in der Konkordanz der politischen Parteien sowie in der Kooperation zwischen Politik und Wirtschaft. ${ }^{258}$ Wörtlich schreibt dazu der ehemalige Regierungsrat und Verfasser einer kurzen historischen Übersicht über Solothurn im 20. Jahrhundert Alfred Wyser: «Eine besonders enge Verflechtung von Politik und Wirtschaft ist ein Charakteristikum des Kantons Solothurn. ${ }^{259}$ In diesem Kräftefeld ist auch die politische Linke fest eingebunden. Der Schriftsteller Peter Bichsel äussert sich dazu unmissverständlich in seinem Vorwort zu «12O Jahre Arbeiterbewegung des Kantons Solothurn»: "Die Geschichte der SP in diesem Kanton ist die Geschichte der FDP. Dem Widerstand gegenüber der Macht folgte immer wieder das Arrangement mit der Macht, und das Arrangement mit der Macht war das Streben nach Salonfähigkeit, und im Salon sind jene geduldet, die sich anständig verhalten, und der Anstand ist freisinnig ... ${ }^{260}$ Es ist denn auch kein Zufall, dass das Friedensabkommen in der Metall- und Maschinenindustrie von 1937 mit den Hauptakteuren Ernst Düby (Direktor von Roll) und dem Chef der Metall- und Uhrenarbeitergewerkschaft Konrad Ilg seinen geografischen Ursprung im ländlich geprägten Gerlafingen hatte.

\footnotetext{
256 Hafner, Die Krise und ihre Bewältigung, S. 54.

257 Lätt, 120 Jahre Arbeiterbewegung des Kantons Solothurn, S. 23 und Kienzle, «Es gibt nur ein Gerlafingen!», S. 173 und 204 .

258 Hafner, Die Krise und ihre Bewältigung, S.12-18 und Wyser, Der Kanton Solothurn im 20. Jahrhundert, S. 18 .

259 Ebd., S.21.

260 Bichsel, Peter: Vorwort, in: Lätt, 120 Jahre Arbeiterbewegung des Kantons Solothurn, S. 12.
} 


\subsection{Die Arbeitsgemeinschaft für Osteosynthese als Geburtshelfer und Partner}

Die Bedeutung der Arbeitsgemeinschaft für Osteosynthese (AO) für die Entwicklung der Medizintechnik am Jurasüdfuss ist in Kapitel 5 ausführlich dargestellt worden. Darüber hinaus haben ihr Selbstverständnis und ihre Funktionsweise den regional stark verankerten Wirtschaftszweig stark beeinflusst. Deshalb gilt unser Augenmerk nun dem Selbstverständnis, den Grundregeln und den Handlungsmaximen dieser Vereinigung von Chirurgen. Unter dem Titel «Eine wissenschaftlich-industrielle Symbiose» hält die Neue Zürcher Zeitung in einer «Sonderbeilage Medizinaltechnik» fest: «Ein Kreditgeber dürfte Mühe haben, den kommerziellen Reiz eines Projektes zu erkennen, bei dem eine Stiftung den Ton angibt, deren Ziel nicht die Gewinnmaximierung, sondern (laut Statuten) «das Studium der Fragen der Knochenbruchbehandlung> ist.» ${ }^{261}$ Tatsächlich war das Hauptmotiv der AO nicht Konkurrenz, sondern Kooperation. Der emeritierte Management-Professor Jean-Pierre Jeannet vermutet, dass - entgegen betriebswirtschaftlicher Logik - das gemeinsame geistige Eigentum und der offene Austausch der eigentliche Geniestreich gewesen sei, der für das Wachstum der AO verantwortlich war. ${ }^{262}$ Maurice E. Müller gab bei der Gründung der Arbeitsgemeinschaft, die durch Forschung, Dokumentation und Schulung der Osteosynthese zum Durchbruch verhelfen wollte und sich ausschliesslich aus Chirurgen zusammensetzte, alle seine Patente (ausser jene für Hüftprothesen) ab und definierte dadurch Prinzipien und Leitlinien. ${ }^{263}$ Wörtlich erklärte er: «I had given this issue a great deal of thought. My gift of intellectual property would ensure the necessary founding for AO Switzerland for the future. This act of giving intellectual property subsequently became a standard of practice for those who belonged to AO. AO surgeons voluntarily transferred new intellectual property that they developed to Synthes AG Chur in order to ensure the growth of welfare of the group and its common goals. $)^{264}$

Entsprechend ihren Zielsetzungen war die AO weitgehend frei von Hierarchien. Freiwilligkeit und Gleichheit kommen bereits in den Begriffen «Arbeitsgemeinschaft» und "Obmann» zum Ausdruck. Der demokratische Umgang stand im Gegensatz zum damals autoritären Stil in Spitälern und ermöglichte eine unver-

\footnotetext{
261 ai: Eine wissenschaftlich-industrielle Symbiose, in: Neue Zürcher Zeitung, Sonderbeilage Medizinaltechnik in der Schweiz 2.12.2003.

262 Jeannet, Leading a Surgical Revolution, S. 302.

263 Ebd., S.117-118.

264 Zit. nach: Schatzker, Joseph: Maurice E. Müller: In his own words, Davos 2018, in: Jeannet, Leading a Surgical Revolution, S.118.
} 
krampfte Fehlerkultur. Der Medizinhistoriker Thomas Schlich sieht in der vielfältigen und offenen Struktur der AO den wichtigsten Grund für die Integration von medizinischer Praxis, Wissenschaft und Industrie. Nach seiner Einschätzung wäre eine solche Arbeitsgemeinschaft in hierarchiebewussteren Gesellschaften wie in Deutschland oder Österreich nicht möglich gewesen. Er bezeichnet die AO als eine Art Bruderschaft ("fraternity»), wie sie sich in der Schweiz über Vereine, Militärdienst, informelle berufliche Verbindungen oft herausbilde. Tatsächlich entstand die Kerngruppe um Müller, Schneider, Bandi, Willenegger und Allgöwer teils auf der Basis von ausserberuflichen Aktivitäten und teils im professionellen Austausch, wobei die Fachleute typischerweise aus kleineren und mittleren Spitälern stammten. Diese Überschaubarkeit ermöglichte einen zwanglosen und unmittelbaren Kontakt («face to face») unter den Akteuren. Unter diesen Bedingungen war es auch möglich, sogar gewollt, dass jüngere Chirurgen ihre älteren Berufskollegen kritisieren konnten. ${ }^{265}$ Das ehemalige AO-Mitglied Urs F.A. Heim bestätigt die Analyse von Schlich, wenn er festhält, dass unter kompetitiven, hierarchischen Strukturen eine langjährige, methodische Zusammenarbeit, wie sie in der AO der Fall war, niemals möglich gewesen wäre. Dasselbe gelte für den Vertrag zwischen der Synthes AG Chur und den Produzenten. Dazu brauche es die in der schweizerischen Tradition verankerte Dialog- und Konsenskultur, bei der «Berufs- oder Standesunterschiede unbedeutend sind». ${ }^{266}$ Das gemeinsame System von Werten, Regeln und Vereinbarungen, der intensive Informationsfluss und das gegenseitige Vertrauen waren zweifellos wesentliche Mosaiksteine für den Erfolg der AO. Rein äusserlich zeigte sich die egalitäre Grundhaltung in der Verpflichtung aller Mitglieder, ihre Operationserfahrungen detailliert zu dokumentieren. Gleichzeitig genossen alle Beteiligten Einsicht in die umfangreiche Dokumentation. Diese Praxis führte - neben der intensiven Schulungstätigkeit - zu einer Dezentralisierung und zur Standardisierung des Wissens und begünstigte die Bildung von stets neuen Netzwerken. ${ }^{267}$

Die AO lieferte den Chirurgen ein integriertes Gesamtpaket von Sets mit Instrumenten, Schrauben, Platten, Büchern, Merkblättern, Kursen, wissenschaftlichen Begründungen. Das war in der Chirurgie neu und zugleich Schlüssel für den Erfolg der AO: "Comparative examination thus underlines the general thesis that control of the materials and aptitude in their application was the key to the successful introduction of osteosynthesis by the AO ... ${ }^{268}$ Dass dieser Erfolg nicht nur medizinische, sondern auch kommerzielle Auswirkungen hatte, unterstrich die Neue

265 Heim, Das Phänomen AO, S. 37-38 und Schlich, Surgery, Science and Industry, S.35-39 und 61.

266 Heim, Das Phänomen AO, S. 209.

267 Ebd., S.114-115 und Schlich, Surgery, Science and Industry, S. 43 und 81.

268 Ebd., S.184. 
Zürcher Zeitung in ihrer Sondernummer zur Medizintechnik: «Diese Standardisierung, die erste ihrer Art in der Knochenchirurgie, ... war auch gut für das medizinaltechnische Geschäft.» ${ }^{269}$

Über den medizinischen und kommerziellen Erfolg hinaus veränderte die AO das Berufsverständnis in der Chirurgie grundlegend. Bis dahin überwog die Meinung, dass die Chirurgie eher eine Kunst als Wissenschaft oder gar Handwerk und folglich entscheidend von der individuellen Genialität geprägt sei. So sah der bekannte Chirurg Hans Ulrich Buff, ab 1961 Professor an der Universität Zürich und Chirurg an der Zürcher Universitätsklinik, den Chirurgen primär als Künstler und weniger als Wissenschaftler. Er kritisierte die Verwissenschaftlichung dieser Disziplin als Kulturzerfall und Amerikanisierung. ${ }^{270}$ Demgegenüber betonte sein Widerpart und Exponent der AO, Maurice E. Müller, dass die Heilung von Frakturen nicht bloss reine Technik oder Wissenschaft, sondern primär medizinische Praxis sei, die durch die Bedürfnisse des Operationssaals definiert werde. Folglich könnten die Fähigkeiten, bei sachgemässer Instruktion, von jedem Chirurgen erworben werden. Die chirurgische Technik sei letztlich mit handwerklichem Können zu vergleichen. Sie werde gleichsam implizit «by doing» erworben. ${ }^{271}$

Die AO-Chirurgen stellten mit ihrer neuen Technik die bisherige Praxis radikal in Frage. In der etablierten Ärzteschaft wurde dies als Provokation empfunden, zumal die Neuerer an regionalen Spitälern praktizierten und keiner unter ihnen eine Professur innehatte. Die Replik von Müller gegenüber Buff, nur Experimente und nicht Ex-cathedra-Meinungen könnten die Osteosynthese widerlegen, verschärfte die Kontroverse und markierte die unüberbrückbaren Positionen. Die geradezu feindselige Haltung des traditionellen Lehrkörpers schweisste die AO-Mitglieder zusammen und stärkte ihr Sendungsbewusstsein. Als Innovatoren hatten sie eine Mission und glaubten an die Überlegenheit ihrer Technik. ${ }^{272}$

Bis heute hat die AO 500000 Chirurgen aus aller Welt geschult. Abgesehen davon, dass sie sich am 3. August 1984 von einer Arbeitsgemeinschaft in eine Stiftung umgewandelt hat, spiegelt ihre Struktur nach wie vor den Primat der Medizin wider. Der achtzigköpfige Stiftungsrat und die 15 Personen umfassende Exekutive setzt sich überwiegend aus aktiven Chirurgen zusammen. ${ }^{273}$

\footnotetext{
269 ai: Eine wissenschaftlich-industrielle Symbiose, in: Neue Zürcher Zeitung, Sonderbeilage Medizinaltechnik in der Schweiz 2.12.2003.

270 Schlich, Surgery, Science and Industry, S. 39-40 und 137.

271 Ebd., S. 67 und 108.

272 Jeannet, Leading a Surgical Revolution, S.110-113 und Schlich, Surgery, Science and Industry, S. 93.

273 Jeannet, Leading a Surgical Revolution, S. 246-248 und 280.
} 


\subsection{Chirurgie, Technik und Industrie arbeiten zusammen}

Der hierarchiefreie, pragmatische Umgang vereinfachte die Kooperation mit den Produzenten. Für eine Chirurgie, bei der praktisches Wissen, Technik und Handwerk im Vordergrund standen, war ein optimales Instrumentarium (Werkzeuge und Platten) zentral. Als einer der Ersten suchte Müller die Nähe zu Ingenieuren und Handwerkern. Es war sein Ziel, gemeinsam mit ihnen benutzerfreundliche Schrauben, Platten und Prothesen aus einwandfreiem Material zu entwickeln. Am Anfang seiner Zusammenarbeit mit Robert Mathys sen. stand die Entwicklung neuer Schraubentypen, leicht gewölbter Metallplatten und neuer Bohrinstrumente. ${ }^{274}$ Auf dieser Basis entstanden schon bald die systematisch aufgebauten Sets mit Instrumenten und Implantaten (siehe S.36 und 38). Die weltweite Überlegenheit der Hilfsmittel und Werkzeuge trug wesentlich zum Siegeszug der AO bei, zuerst in Europa, später und wohl noch entscheidender in den USA, wie Thomas Schlich hervorhebt: "One of the AO's main assets for winning the respect of its American colleagues was the high quality of its equipment [...] This impression was enhanced by the positive image of Swiss precision work ...."275

Dass Chirurgie und Orthopädie mit Technik und Industrie zusammenarbeiten, ergibt sich aus der Natur der Sache. Die Medizintechnik lebt von der Kooperation zwischen der Privatwirtschaft und staatlichen oder privaten Forschungsinstitutionen. ${ }^{276} \mathrm{Neu}$ und spezifisch waren im Rahmen der AO Intensität, Kontinuität und Umfang dieser Zusammenarbeit und die Vielfalt der beteiligten Akteure. Thomas Schlich spricht von einer eigentlichen Symbiose. "So with its specific symbiosis between surgeons and manufacturers, the AO offered exactly what many colleagues had demanded for decades; and they offered it in a ‘package), so to speak, comprising not only the equipment but also user education and the scientific underpinning.» ${ }^{277}$ Auch Hansjörg Wyss legte anlässlich einer Rede vor dem Swiss Economic Forum den Akzent auf die interdisziplinäre Kooperation. Neben ständiger Innovation und Einbezug der Mitarbeitenden auf allen Stufen sei die gemeinsame Lösungsfindung mit Chirurgen ein wesentliches Erfolgsrezept. ${ }^{278}$ Folgerichtig war (und ist) - im Gegensatz zum traditionellen Vorgehen an Universitäten - die For-

274 Schlich, Surgery, Science and Industry, S. 46-48 und Mathys, Robert: Schlossern für die Gesundheit,

in: Betschon/Betschon/Lindecker/Schlachter: Ingenieure bauen die Schweiz, S. 420.

275 Schlich, Surgery, Science and Industry, S.19o.

276 Breiding/Schwarz, Wirtschaftswunder Schweiz, S. 270.

277 Schlich, Surgery, Science and Industry, S. 255.

278 Wyss, Hansjörg Wyss, S. 225. 
schung stark praxisorientiert. Die praktische Anwendung war Ausgangs- und Zielpunkt. ${ }^{279}$ Sei es bei der Dynamic Compressive Plate, neuen Platten aus Titan, der verbesserten Marknagelung, der Ausdehnung auf die Gesichts-, Schädel- und Wirbelsäulenchirurgie - überall halfen Chirurgen von Anfang an mit. Umgekehrt trugen die Praktiker unter anderem zum Erfolg bei, indem sie aus ihrem impliziten ein explizites Wissen machten. ${ }^{280}$ Die ehemalige Solothurner Regierungsrätin Esther Gassler weist ihrerseits auf die kaum vorhandenen Berührungsängste zwischen Chirurgen und Handwerkern hin. Dadurch verschmelze «akademisches Wissen und handwerkliches Geschick». ${ }^{281}$ Noch deutlicher äussert sich der ehemalige AO-Obmann Robert Schneider, der nicht nur die fachlichen, sondern auch die freundschaftlichen Verbindungen vieler Akteure hervorhebt: «Ich bin fest überzeugt, dass der wissenschaftliche Gewinn, die offensichtlichen Verbesserungen der Behandlungsmöglichkeiten und schliesslich die wertvollen menschlichen und freundschaftlichen Kontakte über viele Grenzen hinweg eine sehr positive Bilanz ergeben. Chirurgen und Orthopäden, Grundlagenforscher, Techniker und Produzenten sind Glieder einer Kette, jedes auf das andere angewiesen und überzeugt, mit dem Partner eine glückliche Verbindung eingegangen zu sein.» ${ }^{282}$ Die Arbeitsbeziehungen waren von gegenseitigem Respekt geprägt, im Wissen um die gegenseitige Verstärkung. Als Beispiel unter vielen sei die Laudatio von Maurice E. Müller zum 75. Geburtstag von Robert Mathys erwähnt: «Besonders geschätzt habe ich seinen Enthusiasmus, seinen Einfallsreichtum, sein Einfühlungsvermögen und sein Verständnis für meine Anliegen. ${ }^{283}$ Auch Robert Frigg findet es im Rückblick aussergewöhnlich, dass er 1978 nach seinem Lehrabschluss als Mechaniker in die Technische Kommission (TK) der AO aufgenommen wurde. "Ich hatte Ideen, die Chance Neues auszuprobieren und bald danach die ersten Patente angemeldet. ${ }^{284}$

Die in der AO vereinigten Chirurgen haben bezüglich einer breit abgestützten Zusammenarbeit Pionierleistungen erbracht. Darauf weist Robert Mathys jun. hin, wenn er festhält, dass vor der Begegnung zwischen Maurice E. Müller und seinem Vater die Standesschranken klar abgesteckt gewesen seien. Erst das Durchbrechen dieser Schranken habe die Tür zum Erfolg geöffnet: «Der Ursprung war das Verständnis zwischen Müller und meinem Vater. Sie haben sich trotz verschiedenen Fachsprachen gefunden. Der Dialog wurde immer besser, auch wenn Technologie

\footnotetext{
279 Schlich, Surgery, Science and Industry, S. 95.

280 Ebd., S. 200-207.

281 Gassler-Leuenberger, Esther: Die Bedeutung der medizintechnischen Firmen und von Mathys als Arbeitgeber im Kanton Solothurn, in: RMS Foundation (Hrsg.), Engagement in zweiter Generation, S. 74.

282 Zit. nach: Schneider, Robert: 25 Jahre AO-Schweiz, Biel 1983, in: Heim, Das Phänomen AO, S. 128.

283 Müller, Maurice E.: Laudation, in: RMS Foundation, Seminar zum 75. Geburtstag von Dr. h.c. Robert Mathys, S.162.

284 Gespräch mit Robert Frigg.
} 


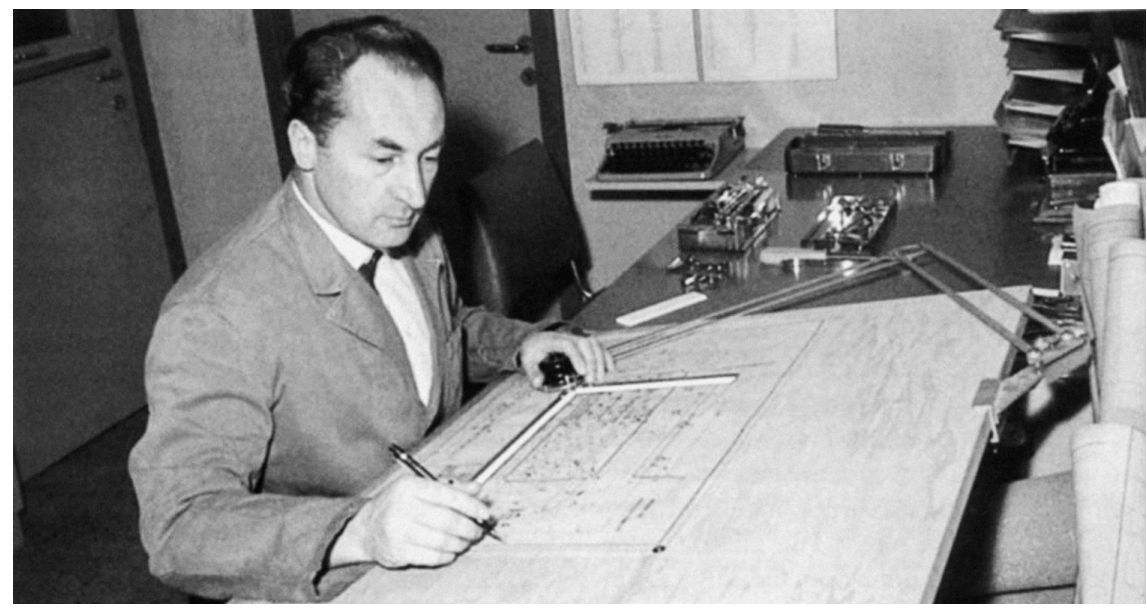

Der Tüftler Robert Mathys sen. am Zeichenbrett

Copyright by AO-Foundation, Switzerland

und Medizin ursprünglich verschiedene Welten waren.» ${ }^{285} \mathrm{Je}$ mehr die von der AO ausgehenden Synthes-Produkte den Weltmarkt eroberten, desto stärker wuchs das Ansehen der Techniker, Entwickler und Produzenten. So wurde Robert Mathys 1974 Dr.h.c. der Universität Bern, erhielt 1992 die Werner-Körte-Goldmedaille der Deutschen Gesellschaft für Chirurgie und wurde 1991 Honorarprofessor der Universität von Venezuela. Robert Frigg seinerseits wurde 2008 Ehrendoktor der Universität Zürich und 2011 der Privatuniversität Paracelsus in Salzburg. Zudem hat er einen regelmässigen Lehrauftrag an der Charité in Berlin. ${ }^{286}$

Die Zusammenarbeit erfolgte sowohl zwischen einzelnen Chirurgen und Firmen als auch institutionalisiert, im Rahmen der AO. So kooperierten Mathys und Straumann ab 1961 eng mit der TK der AO, welche ihrerseits die Bedürfnisse der Produzenten berücksichtigte. Hergestellt wurden bloss Instrumente und Implantate, die von der TK genehmigt wurden. Später waren die Produzenten an der Gründung der AO-Stiftung beteiligt, und danach waren sie Partner des 1992 errichteten AO-Centers in Davos. ${ }^{287}$ Auch an den AO-Schulungen nahmen die Hersteller eine aktive Rolle wahr, indem sie den Umgang mit ihren Produkten instruierten und von den Teilnehmenden Anregungen erhielten. Die Kurse waren in diesem Sinne auch ein Mittel der Qualitätssicherung und Standardisierung. ${ }^{288}$ Angeregt durch

\footnotetext{
285 Gespräch mit Robert Mathys jun.

286 Ebd. und Gespräch mit Robert Frigg.

287 Heim, das Phänomen AO, S.87.

288 Schlich, Surgery, Science and Industry, S. 61 und 69-71.
} 
die AO, führten die Firmen auch eigene, produkteorientierte Schulungen, Workshops und Symposien durch. ${ }^{289}$

In dem Netzwerk innerhalb der Medizintechnik waren sich die Produzenten ihrer Rolle und Bedeutung durchaus bewusst. Kurz und klar kommt dies in einer nach dem Management-Buy-out veröffentlichten Selbstdarstellung der Stratec Medical zum Ausdruck: «Ziel ist es, eine Brücke zwischen Kundenbedürfnissen, wissenschaftlichen Ideen und praktischen Herstellungsmöglichkeiten zu schlagen. Wir sind der Überzeugung, dass interdisziplinäre Teams, die sich aus Ärzten, Ingenieuren, Technikern, Betriebswirtschaftlern und Handwerkern zusammensetzen, die beste Voraussetzung dafür sind.» ${ }^{290}$ Die Produzenten müssen dabei - etwa bei der Herstellung einer Schraubenkassette und eines Druckplattensets - die Arbeitsinhalte der Chirurgie von den Operationsabläufen bis hin zur Knochenbeschaffenheit gut kennen. ${ }^{291}$ Dass sie in diesem Prozess durchaus als selbstbewusste Handwerker/Techniker mitwirkten, mögen die beiden Beispiele von Robert Frigg und Robert Mathys sen. verdeutlichen. Robert Frigg erklärte seinen «Medtech-Virus» mit der Möglichkeit, innerhalb eines faszinierenden, gegenseitigen Geflechts Bestehendes zu optimieren und Neues zu schaffen. Seinen Beitrag schätzte er nicht ohne Berufsstolz ein. "Ich habe die Begabung, etwas anzuschauen und zu sehen, ob es gut oder nicht gut ist. Dasselbe gilt, wenn ich jemandem zuschaue, der ein Instrument in den Händen hält. Das half mir, stur daran zu bleiben, bis ich eine Lösung hatte. Und, ich hatte (und habe) immer eine Lösung.»"292 Ähnlich äusserte sich Robert Mathys sen. nach seinen ersten Assistenzen bei Operationen im Oktober 1958: «Ich erkannte dabei gleich eine ganze Anzahl von Verbesserungen an den Instrumenten.» Und: «Innert weniger Wochen hatte ich 16 Neuerungen erkannt, unter anderem den neuen Schraubenkopf mit Innensechskant.» ${ }^{293}$

Diese Selbsteinschätzung wurde von Maurice E. Müller bestätigt, wenn er betonte, dass Mathys nach dem Besuch von Operationen innert weniger Tage die nötigen Hilfsmittel konstruiert habe. ${ }^{294}$ Mathys selbst ergänzte, dass er in einer zweiten Phase durch die regelmässige Teilnahme bei Operationen eigene Entwicklungen auf den Markt gebracht habe. ${ }^{295}$ Dank ihres Fachwissens waren Unternehmer wie Mathys, Straumann, später auch Wyss in der Lage, die Entwicklungschancen eines Projektes abzuschätzen und kurzfristigen finanziellen Verlockungen zu wi-

\footnotetext{
289 Gespräch mit Robert Mathys jun.

290 Stratec Medical, Im Dienste der Gesundheit, S.18.

291 Gespräch mit Robert Mathys jun.

292 Gespräch mit Robert Frig.

293 Heim, Das Phänomen AO, S. 58.

294 Müller, Maurice E., in: AO Dialogue 14 2001, S.12.

295 Jäggi, Daniela: Robert Mathys arbeitet nach der Devise: Marktlücken erkennen und diese schliessen, bevor es andere tun, Interview mit Robert Mathys, in: Solothurner Zeitung 31.1.1996.
} 
derstehen. ${ }^{296}$ Abgerundet wurde das Selbstverständnis der Verantwortlichen durch eine hohe Wertschätzung gegenüber ihren Mitarbeitern. Dazu zitieren wir nochmals aus der Selbstdarstellung von Stratec Medical. «Bei der Zusammensetzung der Teams hat die persönliche Kompetenz Vorrang vor Ausbildung, Diplomen oder Titeln. Konkret bedeutet dies bei uns, dass zum Beispiel ein Mechaniker sehr eng mit einem Ingenieur, einem Betriebswirtschaftler und einem Arzt in einem Projektteam von Stratec Medical zusammenarbeitet.»297

\subsection{Zwischen Konservatismus und Innovation}

Innovationen basieren auf bestehenden Kenntnissen, Fähigkeiten, Erfahrungen, die für die Entwicklung neuer Produkte oder neuer, optimierter Arbeitsprozesse eingesetzt werden. Von Innovationen ist dann die Rede, wenn sich die Neuerungen auf dem Markt erfolgreich durchgesetzt haben. Oft führen sie zu einem eigentlichen Bruch mit der bestehenden Praxis und können dadurch neue Entwicklungsschritte auslösen. ${ }^{298}$ Sie sind meist mit einem gewissen Risiko verbunden, versprechen aber neben dem zu erwartenden Profit auch neue Erkenntnisse und Überlegenheit gegenüber den Konkurrenten. Wichtige Erfolgsvoraussetzungen für Innovationen sind überbetriebliche Kooperationen, die namentlich auf regionaler Ebene als Katalysatoren für das Innovationspotenzial wirken können. Ebenso entscheidend ist der Austausch mit Kunden, Lieferanten oder Universitäten. ${ }^{299}$

Innovation ist gemäss Robert Mathys jun. eine Konstante in der Medizintechnik. Engineering hat deshalb einen hohen Stellenwert. Neben der Konkurrenz im In- und Ausland fördert auch der Spardruck im Gesundheitswesen die Notwendigkeit von Neuerungen. ${ }^{300}$ So waren zu Beginn des Jahrhunderts 25 Prozent der Produkte von Synthes-Stratec nicht älter als drei Jahre. Im Kanton Solothurn hatte die Medizintechnik 2017 nach der Uhrenindustrie mit Abstand am meisten Patente. Dank der hohen Gewinnmargen (ähnlich wie bei der Uhrenindustrie) können hohe Mittel für Innovationen eingesetzt und entsprechende Risiken eingegangen wer-

296 Perren, Stephan M./Regazzoni, Pietro: Das Familienunternehmen Mathys und die Arbeitsgemeinschaft für Osteosynthese, in: RMS Foundation (Hrsg.), Engagement in zweiter Generation, S. 71-72.

297 Stratec Medical, Im Dienste der Gesundheit, S.11.

298 Gugerli, David / Tanner, Jakob: Wissen und Technologie, in: Halbeisen/Müller/Veyrassat, Wirtschaftsgeschichte der Schweiz, S. 266 und Jeannet, Leading a Surgical Revolution, S.111.

299 Dümmler, Wissensbasierte Cluster in der Schweiz, S. 23-25.

300 Gespräch mit Robert Mathys jun. 
den. ${ }^{301}$ Wie in anderen Branchen sind auch in der Medizintechnik KMU oft besonders innovativ. Ihre Erfindungen werden dann von Grossunternehmen übernommen. ${ }^{302}$ Als Beispiele seien die bereits erwähnte, von Robert Frigg geleitete $41 \mathrm{me}-$ dical und die Firma Mathys nach dem Verkauf der Osteosynthese-Abteilung aufgeführt. Gerade in zielgerichteten Innovationen sieht Letztere ein wesentliches Unterscheidungsmerkmal gegenüber Grosskonzernen. ${ }^{303}$ Wörtlich erklärte Hugo Mathys in der Handelszeitung: "Als KMU sind wir auch weiterhin gezwungen, innovativer und schneller zu sein als die Konkurrenz.»304

Gesamtschweizerisch ist das Umfeld für Innovationen in der Medizintechnik positiv. In der schweizerischen Industrie werden überdurchschnittlich viele Produkte mit hoher Wertschöpfung hergestellt. 2013 nahm die Schweiz beim Anteil von Neuheiten in Prozent des Verkaufsumsatzes europaweit den 1. Rang ein. Sie weist pro Kopf der Bevölkerung doppelt so viele Patente wie Deutschland und über dreimal mehr als die USA auf. ${ }^{305}$ Die spezifischen Voraussetzungen für Innovationen in der Medizintechnik sind günstig, mit Blick auf die allgemeinen Fortschritte in der Technologie, die demografische Entwicklung, den hohen Stand des Gesundheitswesens, die Nähe zur Maschinen-, Uhren- und Pharmaindustrie.. ${ }^{306}$

Gerade wegen seiner Innovationskraft bezeichnete Hansjörg Wyss als damaliger Verantwortlicher von Synthes-Stratec den Standort Schweiz als sehr wichtig. In Oberdorf (BL) verfüge man über ein ausgezeichnetes Team in der Produkteentwicklung, das den Spezialisten in den USA zumindest ebenbürtig sei. Ebenfalls am Beispiel Synthes-Stratec lässt sich zeigen, dass die schweizerische Medizintechnik dank Kundennähe sowie hoher Distributions- und Servicequalität über eine solide Basis für die Umsetzung von Innovationen verfügte (und verfügt). ${ }^{307}$ In der SMTI-Branchenstudie 2016 figurierte die Medizintechnik in der Schweiz bezüglich innovationsfreundlichem Umfeld im Vergleich mit den anderen führenden Staaten USA, Deutschland, Irland und Singapur an zweiter Stelle. ${ }^{308}$ Auch die Verantwortlichen selbst massen den Innovationen einen hohen Stellenwert bei. In einer Um-

\footnotetext{
301 Pictet, Synthes-Stratec, S.19, Graber, MedTech-Landschaft Kanton Solothurn, S. 8 und Bohlhalter, Unruh. Die schweizerische Uhrenindustrie, S. 290-292.

302 Gugerli, David / Tanner, Jakob: Wissen und Technologie, in: Halbeisen/Müller/Veyrassat, Wirtschaftsgeschichte der Schweiz, S. 306 .

303 Palm, Carla: Mathys wehrt sich standhaft gegen Offerten, in: Finanz und Wirtschaft 21.7.2007.

304 Wittwer, Judith: Durch Mark und Bein, in: Handelszeitung 9.1.2014.

305 Borner/Porter/Weder/Enright, Internationale Wettbewerbsvorteile, S. 65 und Strahm, Die Akademisierungsfalle, S. 68.

306 Dümmler, Wissensbasierte Cluster in der Schweiz, S. 6.

307 Erbacher, Felix: Möglichst keinen Knochenkontakt, in: Basler Zeitung 4.4.2002 und Städeli, Markus: Höhere Margen in Sicht, in: Handelszeitung 23.10.2002.

308 Murer Mecattaf/Frey/Schmolders/Biedermann, Schweizer Medizintechnikindustrie, S. 41.
} 
frage erwähnten bei den Herstellern 79 Prozent die Produkteinnovation, 75 Prozent die Lancierung neuer Produkte und 72 Prozent die Verbesserung der Prozesse als prioritär, bei den Zulieferern stand das letztgenannte Postulat an erster Stelle. Bezüglich der Zukunftsherausforderungen setzten die Hersteller den Erhalt der Innovationsfähigkeit mit 83 Prozent der Nennungen an die erste Stelle; bei den Zulieferern schaffte es dasselbe Anliegen mit 79 Prozent auf Platz zwei. ${ }^{309}$

In einer 1986 durchgeführten Analyse der Innovationslandschaft im Kanton Solothurn treten vor allem drei Merkmale hervor. Viele Firmen wiesen einen hohen Exportanteil auf und standen deshalb unter einem starken Innovationsdruck. Dank besseren Zugangs zu Informationen, differenzierterer Organisationsstruktur und besserer Stellung auf den Absatzmärkten waren Grossbetriebe tendenziell innovativer als KMU. Da es im Kanton Solothurn einen Überhang an Arbeitskräften mittlerer Qualifikation und verhältnismässig wenig hoch qualifizierte Arbeitskräfte gebe, so die Autoren der Studie, liege der Akzent bei den Prozess-, weniger bei Produkteinnovationen. ${ }^{310}$ Wörtlich erklärten sie: "Solothurnische Unternehmen konzentrieren sich offenbar mehrheitlich auf ihre technische Kompetenz in der Fertigung und widmen der Entwicklung und/oder rascher Übernahme neuer Produkte und den dafür erforderlichen Marktkontakten weniger Aufmerksamkeit als die schweizerische Industrie.» ${ }^{311}$ Abgesehen vom hohen Exportanteil trifft diese Beschreibung auf die Medizintechnik am Jurasüdfuss nicht zu. Die Bedeutung der KMU für Innovationen wurde in diesem Unterkapitel bereits erwähnt. Was die Prozess- und Produkteinnovationen anbetrifft, lässt sich ein Gleichgewicht, wenn nicht gar eine Dominanz bei Neuerungen von Produkten feststellen. Auf diesen Aspekt wurde bereits mehrmals - zumindest indirekt - hingewiesen. Zur Verdeutlichung beschränken wir uns deshalb auf drei kurze Beispiele von Mathys, Stryker und Ypsomed.

Bereits in den frühen 1980er-Jahren waren Forschung und Entwicklung zentrale Grundpfeiler bei Mathys: "Grosses Gewicht misst die Fabrik Mathys Co. der Entwicklung und Forschung neuer Produkte bei, wofür auch enorme Geldsummen aufgewendet werden.»312 Auch bei der zweiten Generation war der Stellenwert von Innovationen unvermindert hoch. So war Robert Mathys jun. beispielsweise für die neuartige Produktion der Implantate in Titan, die Überarbeitung der Universalmarknägel und für die Optimierung des Gelenkersatzes Protek verantwortlich. ${ }^{313}$

\footnotetext{
309 Ebd., S.5O.

310 Frick/Hollenstein, Innovationskraft und Innovationsverhalten, S. 68-71.

311 Ebd., S.86.

312 kt: Instrumentenwelt der Chirurgie, in: Solothurner Zeitung 6.7.1981.

313 Cotting, Anton: Ausgewählte Beispiele gemeinsamer Entwicklungsarbeiten, in: RMS Foundation (Hrsg.), Engagement in zweiter Generation, S. 24-25.
} 
Im persönlichen Gespräch betonte der Sohn des Firmengründers, dass sich Innovationsfreude, modernste Technologien und konsequente Qualitätsorientierung wie ein roter Faden durch die Firmengeschichte ziehen würden. ${ }^{314}$ Auch die Stryker AG hob schon vor zehn Jahren hervor, dass Preisdruck und der Trend zur Regulierung starke Innovationstreiber seien. Als Schlüsselfaktoren für die Zukunft wurden «Innovation", "Qualitätsbewusstsein» und "permanente Verbesserung der Kosteneffizienz» genannt. ${ }^{15}$ Bei der Ypsomed AG hat Innovation sogar eine industriepolitische Bedeutung über die eigenen Firmengrenzen hinaus. Im Geschäftsbericht 2011/12 erwähnte der Hersteller von Injektionssystemen und Insulinpumpen die regelmässige Finanzierung eines firmeninternen Fonds zur Intensivierung innovativer Untersuchungen im Espace Mittelland. ${ }^{316}$ Auf die Innovationsbereitschaft von Synthes sowie auf den hohen Anteil von Ingenieuren bei allen vier Kernbetrieben der Medizintechnik am Jurasüdfuss wurde bereits mehrfach an anderer Stelle hingewiesen.

\subsection{Kompetente Fachkräfte und gegenseitige Wertschätzung}

Bevor wir die Bedeutung gut qualifizierter Fachkräfte für die Medizintechnik am Jurasüdfuss genauer ins Auge fassen, richten wir den Blick auf das strukturelle Umfeld der Bildung in der Schweiz; im Wissen darum, dass ein hoher Bildungsstand für den Erfolg einer Volkswirtschaft unerlässlich ist. Der Wirtschaftshistoriker Tobias Straumann bringt diesen Kausalzusammenhang auf den Punkt: «Je unternehmerischer und je besser gebildet die Arbeitskräfte sind, desto grösser ist die Wahrscheinlichkeit, dass ein Land reich ist.»"317 Das in der Schweiz stark verankerte und verbreitete duale Berufsbildungssystem ist seit Jahrzehnten ein Garant dafür, dass beim Erwerb beruflicher Qualifikationen Theorie und Praxis optimal verbunden werden. Mittlerweile wird diese Tatsache auch im Ausland als komparativer Vorteil angesehen. Gemäss einer Befragung des International Institute for Management Development stellte eine repräsentative Gruppe von 3000 internationalen Managern die Qualität der beruflich ausgebildeten Fachkräfte an die zweite Stelle der At-

\footnotetext{
314 Gespräch mit Robert Mathys jun.

315 Toggweiler, Andreas: Die Schere kam von ganz oben, in: Solothurner Zeitung 24.6.2009.

316 Ypsomed, Geschäftsbericht 2011/2012, S. 47.

317 Straumann, Tobias: Warum ist die Schweiz ein reiches Land? Eine Antwort aus wirtschaftshistorischer Sicht, in: Die Volkswirtschaft 1/2 2010, S.7.
} 
traktivitätsfaktoren der Schweiz, gleich nach der politischen Stabilität. ${ }^{318}$ Das auf einer Kombination von betrieblicher und schulischer Qualifizierung basierende Ausbildungssystem bewirkt, dass sich Fachkräfte relativ rasch neu qualifizieren oder sich auch «in kompetitive Nischen der industriellen Fertigung» umschulen lassen können. ${ }^{319}$

Im persönlichen Gespräch bestätigte der Ex-CEO von Stryker, Thomas Wahl, die Bedeutung der Berufsbildung auch für den Medtech-Sektor. Das duale Ausbildungssystem generiere ein praxisnahes Know-how. Zusammen mit den Universitäten und Fachhochschulen sei im Umfeld der Medizintechnik «eine geballte Ladung an Wissen vorhanden». Das Qualitätsniveau sei in der ganzen Branche überdurchschnittlich hoch. ${ }^{320}$ Wie wichtig das implizite, verinnerlichte Wissen der Fachkräfte ist, beschreibt der ehemalige Chirurg Urs F.A. Heim, als er in seinem Buch über die AO eine Betriebsbesichtigung bei Mathys schildert: "Nur ein einziger besonders begabter Mann war in der Lage, die Winkelplatten zu biegen, ohne sie zu brechen. Man konnte ihm zusehen, wie er diese in der Gasflamme auf Weissglut erhitzte, dann bei einer bestimmten - nur ihm erkennbaren - Temperatur mit einem Handgriff elegant abbog und so in die gewünschte Form brachte.»321

Berufsleute mit einem ausgeprägten Sinn für Präzision, Disziplin bei handwerklicher Arbeit und Sauberkeit sind für die Schweiz im Allgemeinen und für den Jurasüdfuss im Besonderen charakteristisch. ${ }^{322}$ Hinzu kommt die Fähigkeit, sich laufend auf neue Produkte und Anforderungen einzustellen. «Dieses Umfeld ist die ideale Basis, um Ideen von Ingenieuren und Ärzten in Produkte umzusetzen», hält die Sonderbeilage der NZZ aus dem Jahre 2003 fest. ${ }^{323}$ Gemäss Patrick Dümmler, der eine Dissertation über Cluster in der Medtech-Branche verfasst hat, war die Entwicklung der Medizintechnik stark abhängig von den bestehenden industriellen, von der Uhren- und Maschinenindustrie geprägten Rahmenbedingungen. Wörtlich präzisiert er: "Dazu gehören Kenntnisse in der Mikro- und Nanotechnologie, der Präzisions- und Elektromechanik, der Werkstofftechnologie, der Oberflächenbehandlung sowie im Spezialmaschinenbau.»324 Diese Vorteile machten aus der Sicht zahlreicher Beteiligter die hohen Löhne in der Schweiz wett. Diese Einschätzung bekräftigten die befragten Firmen in der bereits erwähnten Umfrage der Medtech-Branchenstudie 2016. Auf die Frage, warum sie in der Schweiz produzieren,

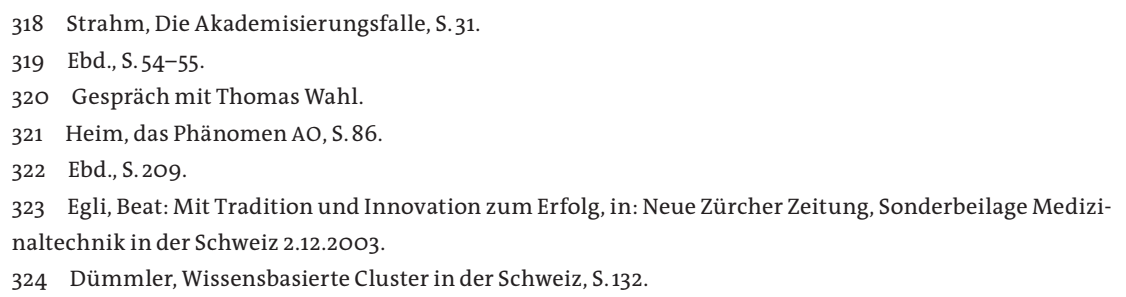


nannten sie das qualifizierte Fachpersonal an erster Stelle. In der Standortanalyse nahm beim Faktor «Fachkräfte» die Schweiz gegenüber Deutschland, USA, Irland und Singapur den Spitzenrang ein..$^{225}$

Trotz des hohen Ausbildungsniveaus leidet die Medizintechnik am Jurasüdfuss - wie schweizweit praktisch alle Produktionssektoren mit hoher Wertschöpfung an einem Mangel an Fachkräften. Fast alle Firmen forcieren deshalb die betriebsinterne Aus- und Weiterbildung, namentlich bei der Vermittlung von implizitem Wissen. Die Tendenz hat mit der Einführung von EDV-gestützten Produktionsprozessen ab Mitte der 1970er-Jahre spürbar zugenommen. Ein wichtiges Ziel ist dabei die vermehrte Ausbildung von Ingenieuren und Technikern. ${ }^{326}$ Stryker, Mathys, Synthes - sie alle unternehmen einiges, um den Fachkräftemangel selber zu beheben. So wies Mathys bereits zu Beginn der 1990er-Jahre auf das eigene Ausbildungskonzept hin. Dieses umfasse mit der Berufslehre, der beruflichen und allgemeinen Weiterbildung drei Schwerpunkte. Für die technischen Berufe stehe eine betriebsinterne Lehrwerkstätte zur Verfügung. ${ }^{327}$ Eine permanente Weiterbildung der eigenen Mitarbeiter war auch für Synthes prioritär. Im Geschäftsbericht 2005 schrieb das Unternehmen: «Die ständige Weiterbildung und Entwicklung unseres Personals ist für das Erreichen unserer Ziele von entscheidender Bedeutung [...] Allein in der Schweiz verbrachte jeder Mitarbeiter 2005 durchschnittlich drei Tage in Ausbildungskursen.» ${ }^{328}$ Einen besonders hohen Stellenwert hat die betriebsinterne Schulung bei der Ypsomed Holding AG: Die starke Gewichtung hängt unter anderem auch mit dem hohen Anteil an angelernten Mitarbeitern ohne Berufsabschluss zusammen. Am Standort Solothurn war 2018 ungefähr die Hälfte von 350 Beschäftigten in der Produktion angelernt, darunter auch ehemalige Berufsleute aus anderen Branchen. Bereits im Stadium der Bewerbung geht die Firma praxisorientiert vor. Sie klärt im Rahmen eines Probetages ab, ob der Bewerber technisch begabt ist. Danach bietet Ypsomed seinen Angestellten eine Nachholbildung (Berufslehre für Erwachsene) für Produktionsmechaniker oder Logistiker an. Diese spezifische Schulung ist auch eine Antwort auf die Qualifikationsveränderungen. Dazu CEO Simon Michel: «Bei der Automation hat der Mensch seinen Platz in der Planung und Steuerung des Prozesses. Wer früher (z. B. als Polymechaniker) ölige Hände hatte, analysiert heute Daten.»329 Neben der Grundausbildung mit zwölf Lehrberufen und internen Weiterbildungen fördert das Unternehmen auch die Höherqualifizierung seiner Berufsleute an Fachhochschulen. Das vielfältige Engagement im Bildungs-

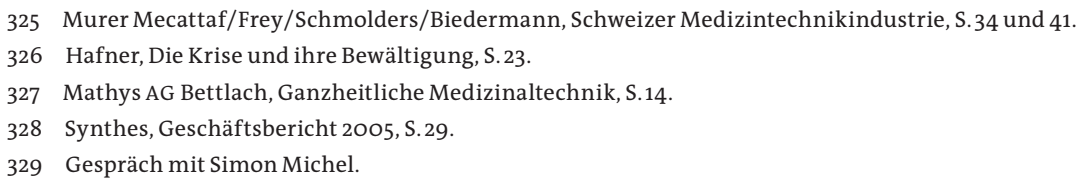


sektor macht Ypsomed unabhängiger vom Arbeitsmarkt. ${ }^{330}$ So wurden etwa im Geschäftsjahr 2014/15 37 Personen in neue Funktionen weitergebildet, davon 24 im Rahmen einer Fachkarriere, zehn innerhalb einer Führungskarriere und drei zu Projektleitern. ${ }^{331}$

Die Schweiz als Hochpreisland kann auf den Weltmärkten nur durch bessere Qualität ihrer Produkte und Dienstleistungen punkten. Der Wirtschaftsfachmann Rudolf H. Strahm betont diesen Fakt und stellt gleichzeitig die Verbindung zum Bildungswesen her: «Hochpreisländer wie die Schweiz, Deutschland, Österreich, aber auch die Länder Skandinaviens haben auf den Weltmärkten nur einen Wettbewerbsvorteil, wenn sie sich auf den Qualitätswettbewerb konzentrieren [...] Als Qualitätsmerkmale gelten zum Beispiel Präzision, Exaktheit, Innovation, Serviceleistungen, Termintreue oder Design - dies sind exakt jene Eigenschaften, die durch das Berufsbildungssystem in Kombination mit gutem, praxisorientiertem Management sichergestellt werden.»332 Das gilt in hohem Masse auch für die Medizintechnik. Bisher war die Fachkompetenz der Arbeitskräfte weitgehend ein Garant gegen Produktionsverlagerungen. Am Jurasüdfuss werden die Fähigkeiten und Qualifikationen der Arbeitnehmer von fast allen Beobachtern als Basis für eine Industrie mit hoher Wertschöpfung und dadurch als Standortvorteil gesehen. ${ }^{333}$ Vor über zehn Jahren begründete der damalige Synthes-Verwaltungsratspräsident Hansjörg Wyss das Festhalten am Produktionsstandort Schweiz damit, dass der Anteil der Personalkosten in der Produktion begrenzt sei und dass die hohen Qualitätsstandards entsprechend geschultes Personal benötigten. ${ }^{334}$ Für die Firma Mathys verwies Robert Mathys jun. auf die Schwierigkeiten seiner Firma beim Ausbau der Produktion in Indien. Mit den Worten «Schweizer Qualität hat ihren Preis», fasste er diese Erfahrung zusammen. ${ }^{335}$ Ähnlich äusserte sich Stryker-CEO Mario Della Casa in der Solothurner Zeitung: «Eine Verlagerung der Produktion in vermeintlich günstigere Standorte ist kein Thema. Wir können uns gegenüber unseren Schwesterbetrieben dank hoher Qualität und einer motivierten Belegschaft behaupten.»336

Das Wissen und die Kenntnisse der Fachkräfte werden von den Arbeitgebern explizit geschätzt. Die Arbeitnehmer ihrerseits identifizieren sich mit ihrer Tätigkeit und dem Betrieb. Auf der Basis dieser gegenseitigen Wertschätzung ist in der Region eine bestimmte Art von Korporatismus entstanden. Unter diesem Begriff ver-

\footnotetext{
330 Ebd.

331 Ypsomed, Geschäftsbericht 2014/2015, S.31.

332 Strahm, Die Akademisierungsfalle, S. 35 .

333 Brand, Andreas, in: Hafner, Die Krise und ihre Bewältigung, S.40.

334 Siebenmann, Nadja: Festhalten am Werkplatz Schweiz, in: Basellandschaftliche Zeitung 29.2.2008.

335 Mathys, Robert jun.: Kostensenkungen sind ein Thema, in: SO Economy 16.6.2011, S.10.

336 Schaible, Franz: Die Firma Stryker ist auf Erfolgskurs, kämpft aber um Fachkräfte, in: Solothurner Zeitung 8.6.2018.
} 
stehen wir im Zusammenhang mit unserer Thematik weder die enge Verflechtung zwischen Wirtschaftsverbänden und dem Staat noch die ständestaatliche Zwangsorganisation von Arbeitgebern und Arbeitnehmern in faschistischen Systemen der 1930er-Jahre. ${ }^{337}$ Vielmehr steht das «liberal-korporatistische System des Schweizer Kapitalismus» ${ }^{338} \mathrm{im}$ Vordergrund. Es ist die Basis für einen Korporatismus auf betrieblicher Ebene, der durch Betriebsgemeinschaft, Identifikation der Mitarbeitenden mit dem Betrieb und wertschätzenden Paternalismus der Arbeitgeber gekennzeichnet ist.

Wie in Kapitel 6.2. angedeutet, hat diese Form der innerbetrieblichen Verbundenheit im Kanton Solothurn und insbesondere am Jurasüdfuss eine lange historische Tradition. Die Industrialisierung ist geprägt von patriarchalen Unternehmern, die politisch und wirtschaftlich ganze Regionen beherrschten wie von Roll in Gerlafingen, Urs und Anton Schild in Grenchen oder Bally in Schönenwerd. 339 So bezahlte etwa die Firma von Roll in der Zwischenkriegszeit im schweizerischen Vergleich niedere Löhne, band die Arbeiter aber durch Zulagen wie betriebliche Altersversicherung oder Familienzuschüsse und Zugaben wie Werkwohnungen oder Familiengärten an den Betrieb. ${ }^{34^{\circ}}$ Besonders markant war der Korporatismus in der Uhrenindustrie, wo die familiären Betriebsstrukturen als Ordnungsfaktor, Garant für soziale Stabilität und Abwehr gegenüber dem Kommunismus gesehen wurden. ${ }^{341}$ Vergleichbar mit der von Roll AG hatte die Uhrenfabrik Lanco in Langendorf eigene Geschäfte und Wohnungen für die Mitarbeiter und die Roamer AG in Solothurn betrieb eine aktive Politik der Aussöhnung zwischen Kapital und Arbeit. ${ }^{342}$ Gemäss Bohlhalter begünstigte die Monokultur eine gewisse Interessenharmonie zwischen Unternehmern und Arbeitern: «In den KMU-geprägten Unternehmen der Uhrenindustrie begegneten sich die Arbeiter und der Patron täglich im Betrieb. Es entstand eine Art Hassliebe.»343 Auch Wolfgang Hafner hebt hervor, dass durch die Dominanz der KMU, in denen anstelle einer Massenproduktion Kleinserien gefertigt wurden, nicht bloss fachliche Fähigkeiten, sondern vielleicht noch ausgeprägter eine durch Zuverlässigkeit, Pünktlichkeit, Disziplin charakterisierte Arbeitsmentalität geschaffen wurde. ${ }^{344}$

Wie aktuell diese Einschätzung ist, unterstreicht Thomas Wahl von der Stryker

337 Farago/Kriesi (Hrsg.), Wirtschaftsverbände in der Schweiz, S.4-5 und S.11.

338 David, Thomas / Degen, Bernard / Mach, André / Brigitte, Studer: Die schweizerische Variante des

Kapitalismus, in: Halbeisen/Müller/Veyrassat, Wirtschaftsgeschichte der Schweiz, S. 827.

339 Lätt, 120 Jahre Arbeiterbewegung des Kantons Solothurn, S. 259.

340 Kienzle, «Es gibt nur ein Gerlafingen!», S. 109 und 259.

341 Donzé, Histoire de l'industrie horlogère suisse, S.193.

342 Scheidegger, Solothurner Pioniergeist, S. 42-44 und 54.

343 Gespräch mit Bruno Bohlhalter.

344 Hafner, Die Krise und ihre Bewältigung, S.115. 
AG: «Unsere Leute haben ein Qualitätsbewusstsein, arbeiten präzis und effizient. Zudem haben wir in der Schweiz stabile politische Rahmenbedingungen, keine mächtigen Gewerkschaften (sic!), eine gute Zusammenarbeit von Politik und Wirtschaft.» ${ }^{345}$ Ähnlich äussert sich Simon Michel, Ypsomed, wenn er feststellt, die Firmen kämen in die Schweiz, weil die Gewerkschaften und dadurch auch der Kündigungsschutz relativ schwach seien. Aus diesem Grunde würden aber auch viele neue Arbeitsplätze geschaffen und Wachstum generiert. Michel betont die gute Zusammenarbeit mit der betriebsinternen Personalkommission und fährt fort: «In einer sozialen Firma, bei der 74 Prozent der Aktien der Familie gehören, sind Gewerkschaften kein Thema. Wir haben keinen Gesamtarbeitsvertrag (GAV). Der GAV ist als Vertragskonstrukt ein Auslaufmodell. [...] Bei einem Familienbetrieb ist das Verantwortungsbewusstsein höher als bei einem managergetriebenen Konzern.»346 Besonders ausgeprägt ist das Selbstverständnis als Familienbetrieb bei Mathys AG. "Wir sind ein typischer Familienbetrieb, in dem der Mitarbeiter eine starke Bedeutung hat. Wir waren (und sind) stets sozial eingestellt - im Gegensatz $\mathrm{zu}$ amerikanischen Betrieben. Unsere Grösse ist überschaubar. Jeder trägt seinen Teil an Verantwortung», ${ }^{347}$ sagt Robert Mathys jun. im persönlichen Gespräch. Diese Grundhaltung hat in der Firma Tradition. Bereits in den 196oer-Jahren erhielten die Mitarbeiter eine Leistungspartizipation von bis zu 10 Prozent Lohnprozenten, jährlich zweimal eine automatische Teuerungszulage, ab drei Jahren Betriebszugehörigkeit eine Treueprämie. Zudem wohnte jeder Vierte in einer günstigen Firmenwohnung. ${ }^{348}$ Dass dieses Prinzip bis in die jüngste Zeit gelebt wurde, wird in der Festschrift zum 70.Geburtstag von Robert Mathys jun. deutlich: «Robert Mathys zielte während seiner Tätigkeit nie auf kurzfristigen Gewinn, sondern hatte immer eine langfristige Optik und hielt so die zentralen Werte des Familienunternehmens aufrecht. [...] Robert Mathys war in diesem Sinne ein Unternehmer ‘alter Schule», der von den Mitarbeitern des Unternehmens nicht nur Loyalität zur Gesellschaft verlangte, sondern selbst auch Loyalität vorlebte.»349

Dem Paternalismus von Unternehmerseite entsprachen bei den Arbeitnehmern eine positive Arbeitseinstellung, Berufsstolz, Motivation, Arbeitszufriedenheit und Identifikation mit dem Betrieb. Diese Eigenschaften sind für eine hohe Arbeitsproduktivität unerlässlich. ${ }^{350}$ Alle Gesprächspartner bestätigten, dass diese

\footnotetext{
345 Gespräch mit Thomas Wahl.

346 Gespräch mit Simon Michel.

347 Gespräch mit Robert Mathys jun.

348 Robert Mathys, Robert Mathys stellt für die ganze Welt Produkte her ...

349 Schenker, Urs: Robert Mathys - Führung der Mathys Medizinaltechnik AG in der Jahrhundertwende,

in: RMS Foundation (Hrsg.), Engagement in zweiter Generation, S. 15.

350 Strahm, Die Akademisierungsfalle, S. 74 .
} 
Einstellung ein Merkmal bei den regionalen Arbeitskräften sei. So erklärte Simon Michel, er habe in Solothurn vor rund 15 Jahren eine vorbildliche Arbeitsmentalität vorgefunden, dies bei einem Ausländeranteil von über 50 Prozent. Insbesondere sei Schichtarbeit am Jurasüdfuss - im Gegensatz zum Kanton Bern - nichts Aussergewöhnliches. ${ }^{351}$ Auch in der Jubiläumsschrift der Ypsotec werden "Qualitätsbewusstsein, Zuverlässigkeit, Ehrlichkeit und Offenheit»352 als spezifische Stärken der Belegschaft hervorgehoben. Die positive Arbeitszufriedenheit und Identifikation zeigten sich bei Ypsomed auch bei einer Mitarbeiterbefragung Ende 2014. Bei einer Rücklaufquote von 84 Prozent betrugen die Zustimmungswerte bei der Verbundenheit mit dem Betrieb (Zukunft) 86 Prozent, bei der Identifikation (Zugehörigkeit) 81 Prozent und bei der Zufriedenheit 75 Prozent. ${ }^{353}$ Entsprechend tief ist bis heute die Fluktuationsrate. Sie beträgt in der Produktion zurzeit weniger als 5 Prozent. Dies ist für den Betrieb allein schon deshalb nutzbringend, weil es in der Regel ein Jahr braucht, bis ein Mitarbeiter ‘seine` komplexe Anlage versteht. ${ }^{354}$ Kontinuität als Erfolgsfaktor hebt auch Mathys hervor. Deren damaliger CEO Arne C. Faisst erwähnte bei einem Podiumsgespräch 2016 einzelne Mitarbeitende bis zu vierzig Dienstjahren. Wörtlich betonte er: «Wir bauen Produkte, die zehn, fünfzehn, zwanzig Jahre dauern müssen, dann sollten die Mitarbeitenden nicht alle drei Jahre wechseln.»355

Wie die Verantwortlichen der drei anderen Grossbetriebe der Medizintechnik am Jurasüdfuss gewichtete auch Hansjörg Wyss Motivation und Berufsstolz seiner Mitarbeiter: «Die Leute müssen stolz sein auf das, was sie machen, sie müssen sehen, dass es sinnvoll ist, was produziert und unter die Leute gebracht wird. Sie müssen das Gefühl haben, dass man nicht nur den Kunden hineinlegen will, wie das in den Banken geschieht.»356 Diese Aussage weist indirekt auf eine berufsethische Dimension, auf die Frage der Sinnhaftigkeit, die im Geschäftsbericht 2005 explizit angesprochen wird. «Was Mitarbeiter jedoch an unserem Unternehmen vor allem reizvoll finden, ist die Befriedigung, zu einer sinnvollen Sache beizutragen - der Verbesserung der Lebensqualität von Menschen auf der ganzen Welt.» ${ }^{357}$ Fast identisch äusserte sich Robert Mathys mehr als 35 Jahre früher, als er festhielt, dass es für jeden seiner Mitarbeiter eine "Quelle der Befriedigung und des Stolzes» sei, «irgendwo auf der Welt einem Kranken Hilfe und Erleichterung (zu) bringen».358

\footnotetext{
351 Gespräch mit Simon Michel.

352 Michel, Simon: Schweiz oder nicht Schweiz, in: 100 Jahre Ypsotec, ohne Seitenangabe.

353 Ypsomed, Geschäftsbericht 2014/2015, S. 36.

354 Gespräch mit Simon Michel.

355 Fluri, Lucien: Weniger als zwei Minuten sollte die Produktion in der Schweiz dauern, in: Solothurner

Zeitung 18.8.2016.

356 Zit. nach Hansjörg Wyss, in: Wyss, Hansjörg Wyss, S. 89.

357 Synthes, Geschäftsbericht 2005, S. 29.

358 Robert Mathys. Es gibt auch eine humanitäre Industrie in der Schweiz.
} 
Im persönlichen Gespräch bestätigte Jesus Fernandez, Verantwortlicher der Gewerkschaft UNIA für die Region Biel-Solothurn, der gewerkschaftliche Organisationsgrad sei in den Medtech-Firmen am Jurasüdfuss verschwindend klein. Er führte das unter anderem ebenfalls auf den Berufsstolz, die Identifikation der Fachkräfte mit dem Betrieb und auf ihre extrem strukturierte Arbeits- und Denkweise zurück. Zudem habe sich die Gewerkschaft zu sehr auf die Uhrenindustrie konzentriert und die Bedeutung der Medizintechnik zu Beginn unterschätzt. 359

\subsection{Ein Medtech-Cluster?}

Die beiden Wirtschaftshistoriker Hartmut Berghoff und Jörg Sydow definieren den Begriff «Cluster» als geografische Konzentration vernetzter Unternehmen mit spezialisierten Zulieferern und Dienstleistern. Merkmale dieses Netzwerks sind zudem die Nähe zu Firmen in verwandten Industrien sowie die Zusammenarbeit mit Institutionen wie Universitäten, Handelsvereinigungen usw. Die relativ stabilen Beziehungen unter den Akteuren generieren Wettbewerbsvorteile. ${ }^{360}$ Diese Faktoren per se bilden aus der Sicht von Patrick Dümmler, Verfasser einer Studie über Clusterbildung in der Medtech-Branche, noch keinen Cluster, sondern sie schaffen das Potenzial dafür. Die Entstehung eines Clusters bedarf folglich einer aktiven Industriepolitik. Diese ist gleichsam ein Balanceakt zwischen «Förderung von Kooperationen und Aufrechterhaltung des Wettbewerbs». ${ }^{361}$ Diese Aussage impliziert - bei aller Gemeinsamkeit der Interessen - ein starkes Element der Konkurrenz unter den Produzenten, wodurch Innovation, Spezialisierung und die Bildung von Zulieferbetrieben überhaupt erst gefördert werden. ${ }^{362}$ In diesem Sinne schliessen sich Wandel und Kontinuität nicht a priori aus. Im Gegenteil: «Ein Berufsraum, der für sich dynamisch ist, stellt Kontinuität durch Innovation und Wandel her.»363

Vor dem Hintergrund dieser Begriffsklärungen soll nun die Frage beantwortet werden, ob es sich bei der Medizintechnik am Jurasüdfuss um ein Cluster handelt. Zunächst lässt sich eine Art Cluster in der verwandten und räumlich nahen Uhrenindustrie feststellen. Der Historiker Pierre Yves Donzé nennt diese Gebilde «districts industriels» (siehe S. 67). Gemäss Breiding und Schwarz ist die «starke regio-

\footnotetext{
359 Gespräch mit Jesus Fernandez.

360 Berghoff/Sydow, Unternehmerische Netzwerke, S. 27 und 31.

361 Dümmler, Wissensbasierte Cluster in der Schweiz, S. 209.

362 Ebd., S. 28 und 49.

363 Mahrer, Handwerk der Moderne, S. 239.
} 
nale Clusterbildung» sogar ein wichtiger Grund dafür, dass die Uhrenbranche existenzielle Krisen immer wieder überwunden habe. ${ }^{364}$ Unter den Gesprächspartnern spricht niemand ausdrücklich von einem Medtech-Cluster am Jurasüdfuss. Auch schriftliche Quellen äussern sich diesbezüglich vorsichtig. Am ehesten können die Aussagen von Thomas Wahl als Hinweis auf ein Cluster verstanden werden. Er wies darauf hin, dass grosse Firmen dorthin gehen, wo bereits Know-how vorhanden sei. Daraus entstünden eine Konkurrenzsituation und eine gegenseitige Stimulierung, die zu Bestleistungen antreibe. Im Interesse der Medtech-Region Jurasüdfuss habe er die Ansiedlung des Life-Science-Konzerns Biogen in Luterbach aktiv unterstützt, indem er Tipps für den Firmenaufbau in der Region weitergegeben habe. Auch der Kanton bzw. die Wirtschaftsförderung nehmen nach seiner Meinung eine aktive Rolle wahr, etwa durch die Umzonung von Land für Firmenneubauten. Thomas Wahl beschreibt die Region denn auch - allerdings etwas allgemein - als "HighTech-Cluster». ${ }^{365}$ Ähnlich äussert sich die Website der Stryker AG. Sie erwähnt einen regionalen Zusammenschluss von Herstellern, Zulieferern, Dienstleistungs- und Forschungsunternehmen, die Fachwissen austauschen und über Betriebsgrenzen hinweg kooperieren. ${ }^{366}$ Seit bald zehn Jahren arbeitet der Kanton Solothurn aktiv im gesamtschweizerischen Medical Cluster mit, in der Absicht, Interaktionen und Neuansiedlungen zu fördern. Trotz dieser engeren Kooperation wird der Jurasüdfuss eher als "nationaler Hotspot für Medizintechnik» oder als "Branchenschwerpunkt» bezeichnet. ${ }^{367}$ Punktuell betreibt die RMS Foundation eine Clusterpolitik, indem sie als Bindeglied zwischen Grundlagenforschung und industrieller Produkteentwicklung tätig ist.

In ihrer Studie über den Wirtschaftsraum «Jurasüdfuss» vermeidet auch die Crédit Suisse den Begriff "Cluster». Obschon die Life-Science-Branche ein jährliches Stellenwachstum von 5,6 Prozent aufweist, benennt auch sie den Jurasüdfuss allgemein als «Standort für Hightech-Produkte». ${ }^{368}$ Sehr dezidiert zur Frage «Cluster - ja oder nein?» nimmt Ypsomed-CEO Simon Michel Stellung. Allein schon wegen ihrer Heterogenität sei die Medtech-Branche keineswegs ein Cluster. Wenn schon gelte diese Bezeichnung eher für die Präzisionsmechanik. Zudem fehle im Kanton Solothurn eine gezielte Clusterpolitik, die Wirtschaftsförderung sei diesbezüglich im Gegensatz zu Swiss Medtech - halbherzig und wenig dynamisch. Eine Art «Best Practice» sei in Solothurn nirgends festzustellen. Allerdings profitiere Ypsomed

\footnotetext{
364 Breiding/Schwarz, Wirtschaftswunder Schweiz, S. 89.

365 Gespräch mit Thomas Wahl.

366 www.stryker.de $>$ standorte $>$ selzach.

367 Toggweiler, Andreas: Schnittstelle zwischen Forschung und Industrie, in: SO Economy 16.6.2011, S.11.

368 Crédit Suisse (Hrsg.), Regionalstudie Wirtschaftsraum Jurasüdfuss, S. 3, 18 und 20.
} 
von gut ausgebildeten Fachkräften und Zulieferern in der Region, auch wenn die Firma als Hersteller von Injektionssystemen nicht zur Orthopädie gehöre. ${ }^{369}$

Fachleute stellen für den breiter gefassten Raum zwischen Basel, Luzern und Biel allenfalls die «Tendenz einer Clusterbildung «Medical Valley` mit Schwerpunkt Jurasüdfuss fest».370 Auch Patrick Dümmler betont, dass eine regionale Förderung nicht dem Aktionsradius der Branche entspreche. Er kommt zum Schluss: «Regionale Medizinaltechnikcluster sind eine Fiktion, ein schweizerischer Medizinaltechnikcluster entspricht besser der Realität. ${ }^{371}$ Zusammengefasst: Die Medizintechnik am Jurasüdfuss weist mehrere Elemente und dadurch auch das Potenzial eines Clusters auf, kann aber nicht als solches bezeichnet werden.

369 Gespräch mit Simon Michel.

370 Simonet, Roland A.: Die starke Medizintechnik als Folge einer logischen Entwicklung, in: So Economy

16.6.2011, S. 22 .

371 Dümmler, Wissensbasierte Cluster in der Schweiz, S.XI. 


\section{Perspektiven der Medizintechnik aus Sicht der Betroffenen ...}

"Hat die Schweizer Medtech-Branche ihren Zenit erreicht?», titelte die NZZ im September 2018..$^{372}$ Mit 15,8 Mrd. CHF Umsatz sei 2017 zwar ein Spitzenwert erreicht und im selben Jahr seien 1500 neue Arbeitsplätze geschaffen worden, hält die NZZ mit Bezug auf die jüngste SMTI-Studie fest. Gleichzeitig weist sie darauf hin, dass die Investitionen in Forschung und Entwicklung zwischen 2015 und 2017 von 9 Prozent des Umsatzes auf 6 Prozent zurückgegangen seien und dass Investitionen vor allem im Ausland erwartet würden. Als Gründe für diese Gewitterwolken am Horizont sieht sie die Sparmassnahmen im Gesundheitswesen, die zunehmende Regulierung und den intensivierten Wettbewerb. 373

Ähnliche Hindernisse nannte Roger Graber von der kantonalen Wirtschaftsförderung Solothurn am Community Event «Medtech Solothurn" vom 20. September 2017. Als besondere Herausforderungen bezeichnete er die strategische Neuausrichtung (Medtech / Biotech / Informations- und Kommunikationstechnologie), Unterwanderung der Standortvorteile durch Globalisierung, neue Regularien, Fachkräfte- und Kompetenzmangel sowie den durch die Frankenstärke verursachten Preis- und Lohndruck. ${ }^{374}$ Der letztgenannte Faktor führt dazu, dass immer mehr Spitalmanager anstelle von Chirurgen über die Art der Implantate entscheiden. Dieser Umstand wiederum hat, in Verbindung mit der verstärkten Regulierung, zur Folge, dass der direkte Kontakt zwischen Forschern/Entwicklern und der Medizin kaum mehr möglich ist. Fast nebenbei bemerkte Robert Frigg im Gespräch, dass ihm nach der Übernahme von Synthes durch J \& J sogar der private Umgang mit befreundeten Medizinern verboten worden sei ... ${ }^{375}$ Ungemach droht der Forschung noch von anderer Seite. Wenn nämlich die Fertigung in der Schweiz heruntergefahren würde, dürfte auch die in den letzten 50 Jahren aufgebaute Technologieführerschaft bald der Vergangenheit angehören. Nach Einschätzung von Robert Mathys jun. sind die marktgängigen Produkte in der Knochenbruchbehandlung ausgereift und auch in der Prothetik ist der Innovationsdruck nicht mehr hoch. Sein Fazit: «Ich war früher immer Optimist, heute sehe ich für den Werkplatz Schweiz Prob-

372 Müller, Giorgio V.: Hat die Schweizer Medtech-Branche ihren Zenit erreicht?, in: Neue Zürcher Zeitung

6.9.2018.

373 Ebd.

374 Graber, MedTech-Landschaft Kanton Solothurn, Folie 10.

375 Wittwer, Judith: Durch Mark und Bein, in: Handelszeitung 9.1.2014 und Gespräch mit Robert Frigg. 
leme kommen. Wir haben in der Medizintechnik keinen Vorsprung mehr. Einfache Produkte sind schon heute «Made in Chinas.» ${ }^{376}$

Regulierung - von allen Beteiligten wird ihre Zunahme als wichtigste Hemmschwelle für die Medizintechnik in der Schweiz eingestuft. In der SMTI-Branchenstudie 2016 bezeichneten die Hersteller die «Hürden bei der Produkteentwicklung» mit 83 Prozent der Nennungen als zweitgrösste Herausforderung. An dritter Stelle, bei den Zulieferern gar an erster Stelle standen die «Qualitäts- und Produktionsanforderungen» (81 Prozent bzw. 80 Prozent der Nennungen).377 Gemäss Robert Frigg werden durch die (regulatorisch bedingte) Trennung von Ärzten und Technikern Innovationen beinahe verunmöglicht. Gespräche - sofern sie überhaupt noch stattfinden - würden ausführliche Rechtfertigungen und Gesprächsnotizen erfordern. Ein schier unüberwindbares Hindernis seien zudem die aus seiner Sicht exzessiven Dokumentationen. Mittlerweile müsse man - gemäss EU-Recht - im Voraus den Nutzen einer Neuerfindung oder Optimierung beweisen. Um dies zu bewerkstelligen, müsse man das Dossier eines konkurrierenden Produzenten mit dem eigenen vergleichen. Kleinere Entwicklungen würden infolge des unverhältnismässigen finanziellen Aufwandes blockiert, grössere Entwicklungen massiv verzögert und verteuert. Robert Frigg rechnet damit, dass ungefähr ein Drittel aller Betriebe schliessen bzw. in den weniger stark regulierten US-Markt abwandern werden. ${ }^{378}$ Auch andere Beteiligte wie beispielsweise Simon Michel rechnen aufgrund der wachsenden Regulierung mit einer Konsolidierung in der Orthopädie. ${ }^{379}$

Uneinheitlich ist die Beurteilung der Zukunftschancen von KMU. Wenig verheissungsvoll hören sich die Prognosen in einem Kommentar des Experten Giorgio V. Müller in der NZZ an: "Das grosse Heer der kleinen Schweizer Medtech-Firmen wird bestenfalls zu Zulieferern der globalen Multis, wird schlimmstenfalls aber geordnet seine Zelte abbrechen und sich andere Tätigkeitsbereiche suchen. Den führenden Medtech-Firmen sollte dies zu denken geben, denn es ist gerade dieses weitverzweigte Ökosystem, das die Branche in der Schweiz so erfolgreich gemacht hat.»380 Demgegenüber sehen Robert Mathys jun. und Simon Michel die Rolle der kleinen und mittleren Betriebe vermehrt als Entwickler für die Grossen. Michel begründet seine Einschätzung damit, dass eingekaufte Entwicklungen die Erfolgsrechnung der Grossfirmen nicht belasten würden. Deshalb gehe der Trend - ganz besonders am Jurasüdfuss - in die Richtung, dass kleine Firmen entwickeln und Grossunternehmen optimieren würden. Überdies sieht Michel die Gefahr,

376 Gespräch mit Robert Mathys jun.

377 Murer Mecattaf/Frey/Schmolders/Biedermann, Schweizer Medizintechnikindustrie, S. 50.

378 Gespräch mit Robert Frigg.

379 Gespräch mit Simon Michel.

380 Müller, Giorgio V.: Der Dreschflegel kommt auf die Medtech zu, in: Neue Zürcher Zeitung 4.4.2016. 
dass nach Abschaffung der heute gültigen Holdingsteuer mehrere internationale Betriebe aus dem Kanton Solothurn abwandern werden, sollte der neue Steuersatz mehr als die in einer Arbeitsgruppe vereinbarten 12,9 Prozent betragen. ${ }^{381}$

Abgesehen von diesem Warnruf, sieht er für sein Unternehmen Ypsomed die Zukunft in positivem Licht. Als «Technologie- und Kostenführer» werde die Firma 2019/2O2O mit dem Pumpengeschäft in den chinesischen Markt eintreten; dies umso mehr, als Diabetes weltweit stark zunehmen werde, von 44 Mio. Menschen, die heute spritzen, auf 72 Mio. im Jahre 2040. Das Geschäft mit den Insulinpumpen werde bereits in den nächsten drei Jahren um jährlich 25 Prozent wachsen. ${ }^{82}$ Dieser Optimismus korrespondiert mit den Prognosen der Kantonalen Wirtschaftsförderung, welche am "Community Event» 2018 festhielt, dass Life Science bezüglich Innovationskraft und Bedeutung die mit Abstand wichtigste Zukunftstechnologie sei, mit der Präzisierung, dass der grösste Teil der Patente aus der Medizintechnik stamme. ${ }^{383} \mathrm{Zu}$ Zuversicht Anlass gibt der Wirtschaftsförderung vor allem die Ansiedlung von Biogen in Luterbach. Bis 2018 hat Biogen bereits 210 Arbeitsplätze geschaffen, mit der Inbetriebnahme der weltweit modernsten Medikamentenherstellungsanlage sollen 2020 weitere 600 hinzukommen. Es handelt sich dabei weitgehend um gut bis hoch qualifizierte Fachleute, die auch aus dem Bereich der Medizintechnik rekrutiert werden. ${ }^{384}$ Auch De Puy Synthes hat - zumindest bishersignalisiert, dass der europäische Hauptsitz in Solothurn bleiben wird. ${ }^{385}$ Gleichzeitig entsteht aber auch eine gewisse Verunsicherung, nachdem neben Le Locle (NE), Raron (VS) und Mezzovico (TI) auch die beiden Solothurner Standorte Bettlach und Grenchen an den riesigen US-amerikanischen Auftragsfertiger Jabil verkauft werden sollen. ${ }^{386}$

Diese globalen Veränderungen deuten darauf hin, dass in den Unternehmen der Medizintechnik die Zeit der charismatischen Einzelkämpfer abläuft und eine verstärkte betriebsübergreifende Kooperation vonnöten sein wird. ${ }^{387}$ Unter diesen neuartigen makroökonomischen Rahmenbedingungen könnte die Sozialpartnerschaft in dem Sektor an Bedeutung gewinnen, gerade auch aus industriepoliti-

\footnotetext{
381 Gespräch mit Robert Mathys jun. und Gespräch mit Simon Michel.

382 Gespräch mit Simon Michel, N.N.: Ypsomed ist auf Wachstumskurs, in: Berner Zeitung 24.5.2017 und Feldges, Dominik: Ypsomed muss stärker auf eigenen Füssen stehen, in: Neue Zürcher Zeitung 24.5.2018. 383 Graber/Müller, Life-Science-Landschaft Kanton Solothurn, Folie 11.

384 Graber, Roger: Vom Uhrenhersteller zum Schweizer Hotspot für Medizintechnik, in: Handelskammerjournal 7.6.2016 und Heim, Roland: Wirtschaft zum Wohle aller, in TOP 100, November 2018, S. 3 . 385 Schaible, Franz: Europa-Hauptsitz des grössten Medtechkonzerns bleibt in Zuchwil, in: Solothurner Zeitung 2.9.2016.

386 Mathys, Urs: Johnson \& Johnson-Standorte Bettlach in neue Hände?, in: Solothurner Zeitung 19.7.2018 und Müller, Giorgio V.: Schweizer Mitarbeiter von J \& J wechseln Firma, in: Neue Zürcher Zeitung 20.7.2018. 387 Müller, Giorgio V.: Die Einzelkämpfer lernen kooperieren, in: Neue Zürcher Zeitung 14.7.2017.
} 
schen Gründen (neue Sichtweisen, politisch stärkeres Gewicht, Verhandlungskultur). Nach Auskunft von Jesus Fernandez hat J\& J am 1. September 2018 die beiden De-Puy-Synthes-Betriebe Grenchen und Bettlach dem Gesamtarbeitsvertrag der Metall-, Elektro- und Maschinenindustrie unterstellt. ${ }^{388}$ 


\section{8. ... und eine Stimme von jenseits des Jura}

Die Kooperation zwischen Chirurgen und Produzenten, das Fachwissen und die Kernkompetenzen der Mitarbeiter, die gegenseitige Wertschätzung von Arbeitgebern und Arbeitnehmern haben wesentlich zur Entwicklung der Medizintechnik am Jurasüdfuss beigetragen. Sind diese Erfolgsfaktoren spezifisch für die erwähnte Region oder gelten sie für die gesamte Medtech-Branche in der Schweiz? Thomas Straumann beantwortet diese Frage in einem Gespräch mit dem Autor.

Straumann ist Gründer und Verwaltungsrat des weltweit vernetzten Zahnimplantate-Herstellers Straumann Group sowie Gründer und Verwaltungsratspräsident von Medartis, einem Produzenten von Implantaten für die Chirurgie von Mund, Kiefer, Gesicht und Extremitäten. Beide Unternehmen sind in Basel, also auf der anderen Juraseite, ansässig.

Thomas Straumann äussert sich im Gespräch über die aktuelle Situation und die Zukunftsaussichten der Medizintechnik in der Schweiz. Zur Veranschaulichung lässt er einige gezielte Informationen aus der Straumann Group und aus Medartis einfliessen.

Das Gespräch fand am 12. August 2020 in Basel statt.

Massgebend für die Entwicklung der Medizintechnik am Jurasüdfuss war die hierarchiefreie Zusammenarbeit zwischen Chirurgen und Produzenten. Begonnen hatte diese Kooperation Ende der 1950er-Jahre zwischen der Arbeitsgemeinschaft für Osteosynthese (AO) und den Produzenten Robert Mathys und Fritz Straumann. Ist diese Verbindung ein generelles Merkmal für die Medizintechnik? Besteht sie heute noch?

Straumann: Die Synergie zwischen Wissenschaft und Industrie ist und bleibt einer der wichtigsten Erfolgsfaktoren. Es geht darum, gegenseitiges Wissen zu vermitteln und umzusetzen. Wir praktizieren das weiterhin unvermindert, sei es bei Straumann, sei es bei Medartis, wo wir ja wieder in der Osteosynthese tätig sind. Alle unsere Aktivitäten basieren auf einem engen Austausch mit Kliniken und Ärzten. Wir stellen unsere Produkte nicht im luftleeren Raum her.

\section{Werden Ihre Fachleute zu Operationen eingeladen?}

Wenn wir neue Ideen entwickeln, sind wir am Operationstisch. Wir sind deshalb namentlich im Entwicklungsprozess regelmässig dabei, um zu beobachten, wie unsere Produkte eingesetzt werden. Auf diese Weise erkennen wir mögliche Schwachpunkte am besten. Ist es eine Frage des Handlings oder des Designs ei- 
ner Platte oder Schraube? Viele Erkenntnisse gewinnen wir auch bei Kadaverkursen. Diese sind für uns ebenso wie für die Ärzte extrem wichtig.

Kurz, das direkte «Arbeiten am Patienten» ist absolut matchentscheidend.

Robert Frigg hat demgegenüber erklärt, dass der direkte Kontakt mit den Chirurgen wesentlich erschwert würde.

Frigg ist bzw. war viele Jahre mit Synthes verbunden. Seine Einschätzung mag dadurch beeinflusst sein, dass zwischen Synthes und der AO die Verbindung mittlerweile lockerer geworden ist. Zeitweise wurde die AO ja fast ausschliesslich durch die Synthes (über die Lizenzvergabe) finanziert. Mit der Übernahme durch Johnson \& Johnson hat sich die Kooperation abgeschwächt. Das bestätigen uns auch ehemalige Synthes-Kunden, die heute mit Medartis zusammenarbeiten.

Bei uns ist die Kooperation zwischen Wissenschaft und operativem Geschäft nach wie vor von zentraler Bedeutung. So entstanden früher die Produkte: Stehen am Tisch, Zeichnen, Diskutieren, Umsetzen. Prototypen entwickeln. Abgesehen von den aktuellen technischen Hilfsmitteln ist die Vorgehensweise im Kern gleich geblieben.

Vor vierzig Jahren gründete mein Vater Fritz Straumann zusammen mit Prof. André Schroeder das International Team for Implantology (ITI). Es handelt sich dabei um einen durch die Funktionsweise der AO inspirierten weltweiten Zusammenschluss von Wissenschaftlern und Industriellen. Die Partnerschaft zwischen der Straumann Group und ITI ist bis zum heutigen Tag sehr intensiv. Medartis seinerseits kooperiert eng mit der International Bone Research Association (IBRA), die wir unter den exakt selben Vorgaben gegründet haben.

Der Zweck solcher Zusammenschlüsse bleibt unverändert: Gemeinsames Weiterentwickeln von Systemen, Vereinfachen von Lösungen im Interesse von Patienten und Ärzten sowie Aus- und Weiterbildung. Auch dieser letztgenannte Tätigkeitsschwerpunkt ist extrem wichtig, weshalb sowohl ITI als auch IBRA darauf grossen Wert legen. Das vielfältige Zusammenwirken von Wissenschaft und Praxis generiert stets neue Ideen und ist deshalb ausgesprochen innovativ.

Verweilen wir kurz bei den beiden Firmen Straumann Group und Medartis. Nach dem Management-Buy-out 1990 haben Sie bei Straumann auf die orale Implantologie fokussiert, die vor der Trennung ungefähr 10 Prozent des Umsatzes ausmachte. Heute ist die Straumann Group der weltweit führende Anbieter in der Implantologie. Wie erklären Sie sich diese rasante Entwicklung?

Die Zahnmedizin erlebte damals eine starke Expansion. Unser Produkt war komplett anders als die bisherigen Systeme. Wir setzten unsere Implantate 
nicht auf Knochenkammhöhe, sondern durch die Schleimhaut hindurch. Dadurch war bzw. ist nur eine einzige Operation erforderlich. Das hat uns einen entscheidenden Konkurrenzvorteil verschafft. Zudem setzte sich die orale Implantologie generell durch. Mit neuen Entwicklungen konnten wir bei diesem Aufwärtstrend mithalten.

Hilfreich war zudem die Konzentration des Unternehmens auf Zahnimplantate. Dieses Geschäft war bisher neben der Osteosynthese marginal. Ich und meine Geschwister wollten aber nach dem Tod meines Vaters die Straumann Holding nicht in der bestehenden Form übernehmen.

So bot mir die Zahnchirurgie die Chance, selber etwas Neues aufzubauen, zumal ich dafür die richtigen Fachleute hatte. Geholfen hat uns auch unsere Risikobereitschaft, etwa bei der Expansion in die USA. Durch Innovationen in der Implantologie, neue Produkte, neue Oberflächenentwicklung, neue Elemente in der Prothetik gelangten wir an die jungen Oralchirurgen. Einer von ihnen war Daniel Buser, Professor für Oralchirurgie an der Universität Bern. Die Zusammenarbeit mit ihm war für uns wesentlich.

Wie sind Sie mit Medartis verknüpft?

Ich bin mit der Osteosynthese gross geworden. Das Gebiet hat mich seit jeher fasziniert. 1992/93 wollten wir bei Straumann ein neues Standbein errichten. Nachdem wir uns auf die Zahnmedizin spezialisiert hatten, lag die Kieferchirurgie am nächsten, zumal wir bereits mit entsprechenden Fachleuten zusammenarbeiteten. Von der im Neurobereich tätigen Firma Elektra aus Freiburg i. B. erwarben wir ein komplettes Maxillo- und Craniofacialsystem. Der Zeitpunkt war allerdings nicht gelegen, da Straumann zur selben Zeit in den Vorbereitungen für den Börsengang steckte. Aus diesem Grunde habe ich zusammen mit Willi Miesch 1997 Medartis gegründet. Zunächst benutzten wir die Vertriebskanäle von Straumann, doch für die Aussendienstmitarbeiter war es schwierig, Plattensystem plus Zahnimplantate gleichwertig zu verkaufen. Folglich schufen wir bei Medartis einen völlig unabhängigen Vertrieb.

Auch die Produktion bauten wir von Grund auf selber auf. Wir konnten uns dabei auf die Décolletage-Kenntnisse im Oberbaselbiet (Bretzwil) stützen. Schritt für Schritt haben wir uns auf der Landkarte der Osteosynthese einen guten Platz erworben. Heute ist der ganze Betrieb - Forschung und Entwicklung sowie die Produktion - in Basel angesiedelt. Mittlerweile haben wir rund 600 Mitarbeiter.

Die Gründung von Medartis war der Weg zurück zu unseren Ursprüngen, zur Osteosynthese. Mit Kopf, Hand, Radius, Ellbogen, Schulter, Schlüsselbein und dem kompletten Fusssystem decken wir ein breites Spektrum ab. Zurzeit erweitern wir unser Aktionsfeld in Richtung Tibia und Sprunggelenk. Weil wir - wie 
erwähnt - eng mit den Chirurgen zusammenarbeiten und deren Ideen umsetzen, sind wir bei unseren Kunden sehr geschätzt.

Gemäss dem Medizinhistoriker Thomas Schlich ist die hierarchiefreie Zusammenarbeit zwischen Chirurgen und Produzenten ein schweizerisches Alleinstellungsmerkmal, das beispielsweise in Deutschland nicht möglich wäre. Teilen Sie diese Meinung?

Zum Teil. Unsere Form der Kooperation funktioniert in Deutschland auch, da gibt es einige Beispiele. Aber es sind auch sehr viele Alleingänge erkennbar. Unsere Kooperation mit der ITI hat ihren Ursprung in der Schweiz, aber auch zum Teil in Deutschland.

Damit sind wir zurück bei den Erfolgsfaktoren. Zu diesen gehört auch das im internationalen Vergleich hohe Qualifikationsniveau der Fachkräfte in der Schweiz.

Das trifft immer noch zu. Unsere Firmen befinden sich am Dreiländereck und profitieren von hervorragenden Mitarbeitern aus dem süddeutschen Raum. So ist Freiburg i. B. seit langem ein Medtech-Schwerpunkt mit ausgeprägtem Fachwissen. Auch aus dem Raum Tuttlingen kommen Fachleute zu uns.

Tuttlingen ist ein ehemaliges Uhrenzentrum. Wie beurteilen Sie die Gemeinsamkeiten zwischen Medizintechnik und Uhrenindustrie?

Die gemeinsamen Kernkompetenzen sind unübersehbar. Auch wir haben uns in Uhrenregionen niedergelassen. Die Fachleute sind dort präzises Arbeiten gewohnt, sie wissen, was Toleranzen bedeuten.

Als im Laufe der 1980er-Jahre die orale Implantologie in Schwung kam, hat Straumann als Produktionsstandort St.Imier im Berner Jura gewählt. Unsere Firma hatte dort schon lange vorher für die Uhrenindustrie die Aufzugs- und Spiralfeder Nivaflex entwickelt und laminiert. Nachdem die Laminage eher rückläufig war, haben wir in St. Imier die gesamte Produktion für Zahnimplantate aufgebaut, wobei wir auf bestehende Décolletage-Kenntnisse zurückgreifen konnten. Auch nach der Expansion blieben wir in dieser Region und errichteten im benachbarten Villeret ein neues Werk.

Ich spüre Ihre Wertschätzung gegenüber den Fachleuten. Diese Wertschätzung ist mit ein Grund dafür, dass sich die Medtech-Arbeitnehmer verhältnismässig stark mit ihrem Beruf und ihrem Arbeitgeber identifizieren.

Das kann ich bestätigen. Wir stellen in der Medtech-Branche eine hohe Loyalität, ein ausgeprägtes Commitment zu Unternehmen und Produkt fest. Indem wir viele Personen selber aus- und weiterbilden, entsteht eine starke Bindung zum Betrieb. Auch Mitarbeiter, die aus anderen Betrieben zu uns kommen, sind meist keine Jobhopper. 
Die Anstellungsdauer hängt natürlich immer auch von der allgemeinen Konkurrenzsituation ab. Generell haben wir jedoch, etwa bei Medartis, langjährige Mitarbeiter. Die Fluktuationsrate ist vergleichsweise sehr niedrig.

Trotz hohem Lohnniveau ist die Medizintechnik nach wie vor in der Schweiz verankert, selbst in der Produktion. Was sind die Gründe?

Gerade in der Medizintechnik wird grosser Wert auf das Label «Swiss made» gelegt, sei es in China, Brasilien oder den USA. Die recht hohen Margen der Produkte erlauben es zudem, den Standort Schweiz zu halten. Eine gewisse Rolle spielt auch das Know-how, doch lässt sich dieses heutzutage ins Ausland transferieren, um Produkte von gleicher Qualität herzustellen.

Die Schweiz als Standort von Forschung, Entwicklung und Produktion bedeutet uns viel. So werden wir mit Medartis in Basel bleiben, auch wenn wir in den süddeutschen Raum übersiedeln könnten. Wir sehen einen Vorteil darin, alles unter einem Dach zu vereinigen.

Die Straumann Group hat Produktionsstätten in USA und Brasilien, nur handelt es sich dabei um andere Dimensionen. Dank Fachleuten aus der Schweiz haben wir in Brasilien eine mit der Schweiz vergleichbare Qualität hochgefahren. Auch die Produktion in den USA ist mit jener von Villeret identisch. Das Ganze ist eine Kapazitätsfrage. Der Markt in den USA ist derart gross, dass wir ihn nicht von der Schweiz aus abdecken könnten. In China wiederum bleibt uns keine andere Wahl. Dort müssen wir eher aufpassen, dass das Know-how nicht irgendwohin abfliesst.

Alles in allem: Die Schweiz bleibt wichtig. Wir haben die Fachleute, wir haben eine gute Ausbildung. Es gibt keinen Grund wegzuziehen.

Sie erwähnen die Ausbildung. Deutschland und die Schweiz haben beide das duale Berufsbildungssystem. Haben Sie auch Angestellte aus dem benachbarten Frankreich?

Ja, aber vorwiegend in der Produktion. In Villeret arbeiten bei uns viele Grenzgänger, die - vor dem Hintergrund der Uhrenindustrie - Erfahrungen in der Décolletage mitbringen. Sie sind bei der Bedienung von komplexen Maschinen versiert, ihr Spektrum ist aber begrenzter als bei den schweizerischen und deutschen Fachleuten.

\section{Wie weit sind Sie von den Regulierungsmassnahmen der EU betroffen?}

Regulierungen wird es immer geben, dem können wir uns nicht entziehen. Die neue Medical Device Regulation (MDR) der EU ist strenger als entsprechende Kontrollsysteme aus den USA oder Brasilien. Früher setzten die USA und Brasilien die Massstäbe, heute ist es Brüssel. Bedingt durch COVID-19 ist die EU-Regulierung verzögert worden, doch werden wir nicht darum herumkommen. Wir 
verstehen diese oder ähnliche Entwicklungen immer als Chance. Vielleicht können wir dadurch unsere Prozesse optimieren und uns von der Konkurrenz noch besser absetzen.

Tatsächlich sind die MDR-Vorschriften ausserordentlich detailliert, bis ein Produkt auf den Markt gelangen kann. Das wird kleineren Firmen das Genick brechen, viele Startups werden scheitern. Sie werden finanziell auf der Strecke bleiben, zumal die Innovationsprozesse verlangsamt werden. Möglicherweise besteht da für uns die Chance, beim einen oder anderen Technologie einzukaufen.

"Hat die Schweizer Medtech-Branche den Zenit erreicht?» Was halten Sie von diesem NZZ-Titel vom September 2018 ?

Ich beantworte diese Frage mit einem klaren Nein. Innovation wird es immer geben. Die Digitalisierung wird uns sicher während der nächsten zehn Jahre beschäftigen. Die computergesteuerte Operationstechnik wie die Grundmaterialien lassen viel Entwicklungsraum offen. Die Systeme vereinfachen, die Zeit auf dem Behandlungsstuhl verringern und dadurch massiv Kosten einsparen, dem Operationspersonal Hilfsmittel geben, damit es auch hoch komplexe Fälle auf einfache Art lösen kann - das sind heute die Herausforderungen. Nicht nur die Produktentwicklung, sondern auch die gesamte Aus- und Weiterbildung steht vor neuen Aufgaben. Nein, der Zenit ist bei weitem nicht erreicht.

Robert Mathys jun. sieht in der Frakturbehandlung und in der Prothetik eine Innovationsgrenze.

Das sehe ich anders. Bereits die Zusammenarbeit zwischen Synthes und AO bewegte sich auf einem hohen Niveau. Damals haben wir von Stahl auf Titan umgestellt. Das war ein Entscheid von grosser Tragweite, man glaubte, den Peak erreicht zu haben.

In den letzten zwanzig Jahren hat jedoch nochmals ein enormer Innovationsschub stattgefunden, neue Produkte sind auf den Markt gekommen.

Wir müssen für das Neue bereit sein, das Bestehende immer wieder hinterfragen, in Zusammenarbeit mit der Wissenschaft Alternativen entwickeln. Das gilt für alle Bereiche, auch für die Osteosynthese und für die Traumatologie. Wir selber haben einiges in der Produktepipeline. Ich sehe der Zukunft optimistisch entgegen. 


\section{Fazit}

Im Laufe von gut sechzig Jahren ist der Jurasüdfuss zu einem Schwerpunkt der Medizintechnik geworden. Das zeigt sich allein schon darin, dass in dieser Region die überwiegende Mehrheit der 6200 Medtech-Beschäftigten des Kantons Solothurn tätig ist. Die wichtigsten Teilbereiche sind dabei Traumatologie und Orthopädie. Drei der exemplarisch ausgewählten vier Betriebe, Mathys, Synthes (heute: De Puy Synthes) und Stryker, decken dieses Teilgebiet der Medizintechnik ab. Ypsomed, die vierte Firma, nimmt im Bereich der Injektionstechnik und der Insulinpumpen eine weltweit sehr starke Position ein. Die vorgenommene Auswahl bildet das Spektrum der Medizintechnik am Jurasüdfuss repräsentativ ab. Neben ihrer quantitativen Bedeutung arbeiten diese vier Unternehmen intensiv mit zahlreichen, teilweise hoch spezialisierten Zulieferern zusammen, die ihrerseits rund ein Drittel aller regionalen Medtech-Arbeitsplätze zur Verfügung stellen. Zudem handelt es sich um je zwei Familienbetriebe (Mathys, Ypsomed) und zwei Niederlassungen von globalen Grossunternehmen (De Puy Synthes, Stryker).

Die Annahme, dass die Medizintechnik auf Fachkompetenzen von Arbeitskräften in der viel länger ansässigen Uhrenindustrie zurückgreifen konnte, hat sich bestätigt. Erwähnt seien vor allem die Präzisionsmechanik und Montage. Diese weithin unbestrittene Tatsache wird allerdings von Robert Mathys jun. und Robert Frigg, zwei Ingenieuren bzw. Technikern aus der Medizintechnik, in dem Sinne relativiert, dass sowohl die einzelnen Tätigkeiten wie auch die zu bearbeitenden Materialien in der Uhrenindustrie und der Medizintechnik nicht identisch seien. Aus dieser Einschätzung folgern wir, dass die Verwandtschaft der beiden Branchen weniger durch die spezifischen Tätigkeitsprofile, sondern viel stärker durch die Arbeitsmentalität definiert ist. Diese wird durch strukturiertes, genaues Arbeiten generiert und gefestigt.

Die Arbeitshypothese, wonach «die stabilen Beziehungen zwischen Arbeitgebern und Arbeitnehmern" entscheidend zum Erfolg der Medizintechnik am Jurasüdfuss beigetragen haben, trifft in verschiedener Hinsicht zu. Die Stichworte sind unter anderem «Zusammenarbeit über Standesgrenzen hinweg», «Wertschätzung der menschlichen Arbeitskraft», "Innovationsfähigkeit» und «Identifikation mit dem Betrieb». Das Prinzip «Kooperation statt Konkurrenz» gilt zunächst für die AO, die zusammen mit begabten Handwerkern und Technikern den Grundstein für den neuen regionalen Industriezweig gelegt hat. Die AO als Zusammenschluss von Chirurgen funktionierte weitgehend hierarchiefrei und pragmatisch. Sie betrachtete die Chirurgie nicht als Kunst, sondern primär als Handwerk, das sich sys- 
tematisch schulen und dokumentieren lässt. Diese Grundhaltung erleichterte den Zugang zu hoch qualifizierten Fachleuten und Tüftlern aus Gewerbe und Industrie. Die Zusammenarbeit wurde als notwendige Basis für Neuentwicklungen gesehen. Darüber hinaus war sie begleitet von gegenseitiger Wertschätzung. Für Maurice E. Müller, Robert Mathys und Hansjörg Wyss stand das Wissen und Können ihrer Mitarbeiter im Zentrum. Der Wert des praxisnahen dualen Berufsbildungssystems sowie der permanenten betriebsinternen Schulung war den Arbeitgebern bewusst. Diesen Werten entsprach ein ausgeprägtes Selbstbewusstsein der Fachkräfte, die ihre wichtige Rolle im Gesamtprozess durchaus einzuschätzen wussten. Zusammengefasst lässt sich feststellen, dass die hohe Fachkompetenz auf allen Stufen ein zentraler Erfolgsfaktor war (und ist). Das zeigt sich beispielsweise darin, dass Stryker Selzach innerhalb eines Weltkonzerns bei der Osteosynthese zum Zentrum der Herstellung und Entwicklung geworden ist; dies - wohlverstanden - im Hochlohnland Schweiz.

Ein weiterer wichtiger Mosaikstein für die Erfolgsgeschichte der Medizintechnik am Jurasüdfuss ist eine Form von Korporatismus, der sich bei den Arbeitgebern in wertschätzendem Paternalismus, bei den Arbeitnehmern in ausgeprägter Identifikation mit Beruf und Betrieb äussert. Diese Identifikation wird bei vielen Beteiligten durch das Bewusstsein verstärkt, mit den Produkten und Dienstleistungen zahlreichen Menschen zu helfen - also durch ein gleichsam berufsethisches Element.

Formulierungen wie "Hierarchiefreiheit und Einebnen der Standesunterschiede», «Identifikation mit der Firma», "Arbeitsdisziplin und Präzision», «Kooperation statt Konkurrenz", "Arbeitsgemeinschaft» mögen als helvetisch-altbacken erscheinen. In ihrer Kombination werden sie auch von zahlreichen ausländischen Fachleuten tatsächlich als schweizerisches Alleinstellungsmerkmal und als entscheidende Grundlage für den Erfolg der Medizintechnik in der Schweiz betrachtet. Sie sind kein Widerspruch zu Innovationen, vielmehr fördern sie diese auf allen Ebenen.

Alle bisher erwähnten Erfolgsfaktoren gelten für die Medtech-Branche in der ganzen Schweiz. Sie sind am Jurasüdfuss besonders stark ausgeprägt. Bis heute hat der industrielle Sektor in dieser Region mit einem Drittel der Beschäftigten einen weit grösseren Stellenwert als im gesamtschweizerischen Durchschnitt. Dank einer langen Tradition in der Mikromechanik werden dort überdurchschnittlich viele Produkte mit hoher Wertschöpfung entwickelt und hergestellt. Auch Paternalismus, traditionelle Arbeitstugenden und Identifikation mit dem Betrieb haben in dieser KMU-geprägten Gegend historisch tiefe Wurzeln.

Die Arbeitshypothesen sind bezüglich der Fachkenntnisse, der Industrietradition und der Arbeitgeber-Arbeitnehmer-Beziehungen vollauf bestätigt worden. Was die Clusterbildung anbetrifft, kann am Jurasüdfuss indessen nicht von einem 
Medtech-Cluster gesprochen werden, eher von einem Schwerpunkt. Zwar ist mit der Konzentration verwandter Betriebe sowie mit spezialisierten Zulieferern und Dienstleistern ein Clusterpotenzial teilweise vorhanden, was aber vor allem fehlt, ist eine aktive Clusterpolitik. Zudem ist die Medizintechnik als solche zu heterogen und die Region für die Entstehung eines Clusters zu begrenzt.

In der Studie wird bewusst auch die Bedeutung von Einzelpersonen hervorgehoben; im Wissen darum, dass es einen bestimmten Kontext braucht, damit individuelles Denken und Handeln geschichtlich wirksam werden kann. Im Rahmen eines dialektischen Entwicklungsprozesses beeinflussen Individuen ihrerseits das kontextuelle Umfeld. Mit der zunehmenden Globalisierung könnte in Zukunft allerdings der Einfluss charismatischer Persönlichkeiten schwinden. Die verstärkte Regulierung und der Kostendruck im Gesundheitswesen haben die persönlichen Kontakte zwischen Ärzten und Produzenten und damit einen zentralen Pluspunkt in der Medizintechnik bereits spürbar abgeschwächt. Es wird sich weisen, ob neue Formen der Kooperation entstehen. Dieser eher skeptische Blick in die Zukunft von regionalen Protagonisten wird jenseits des Jura nicht geteilt. Der Basler Medizintechnik-Exponent Thomas Straumann nimmt eine unvermindert enge Kooperation zwischen Wissenschaft und Industrie wahr. Die zunehmenden Regulierungen und den Kostendruck im Gesundheitswesen sieht er primär als Ansporn für weitere Innovationen. 


\section{Anhang}

\section{Anhang 1: Solothurner MedTech-Unternehmen}

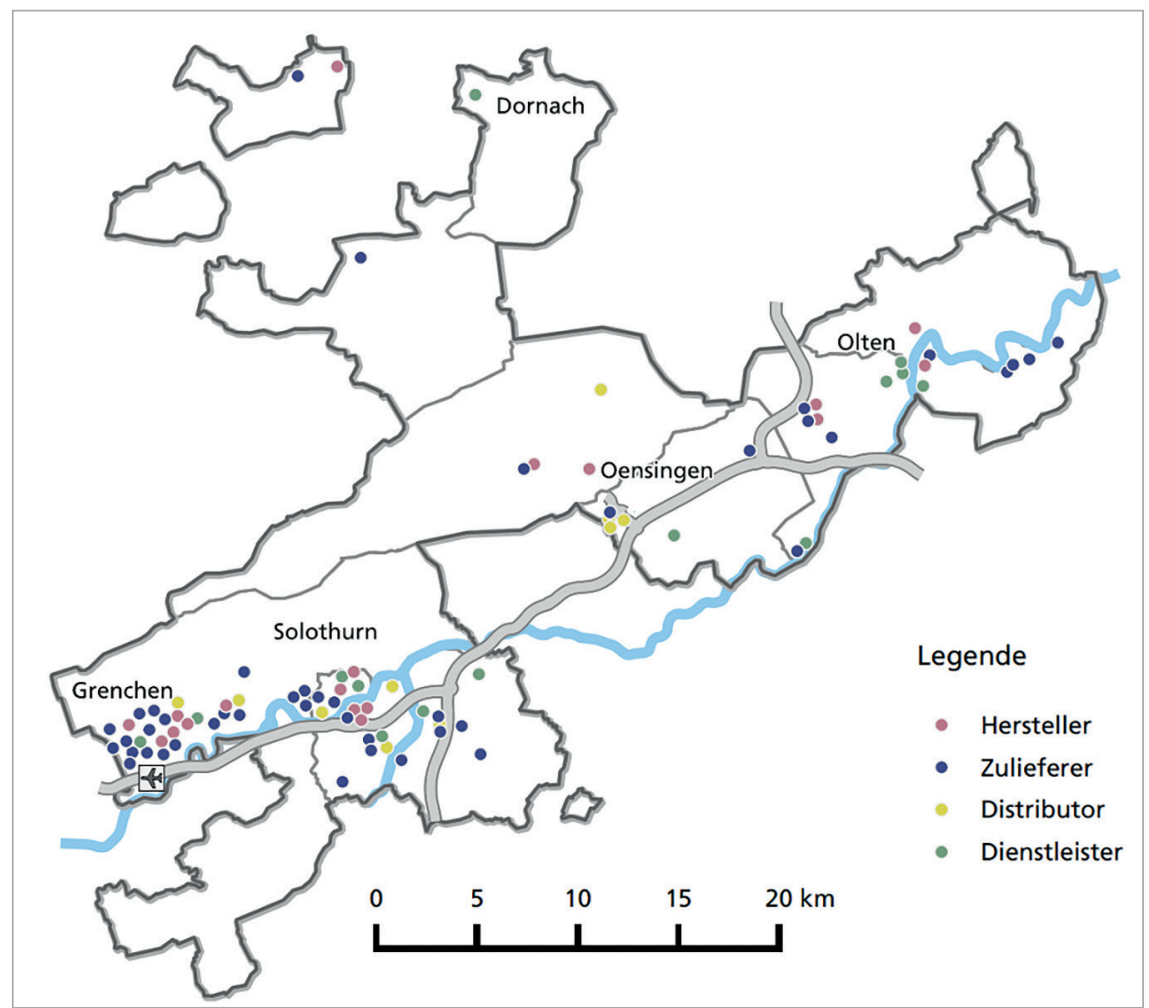

Quelle: Wirtschaftsförderung des Kantons Solothurn, Solothurner Life-Science-Industrie 2018 
Anhang 2: Beschäftigte in der Medizintechnik im Kanton Solothurn 1995-2016

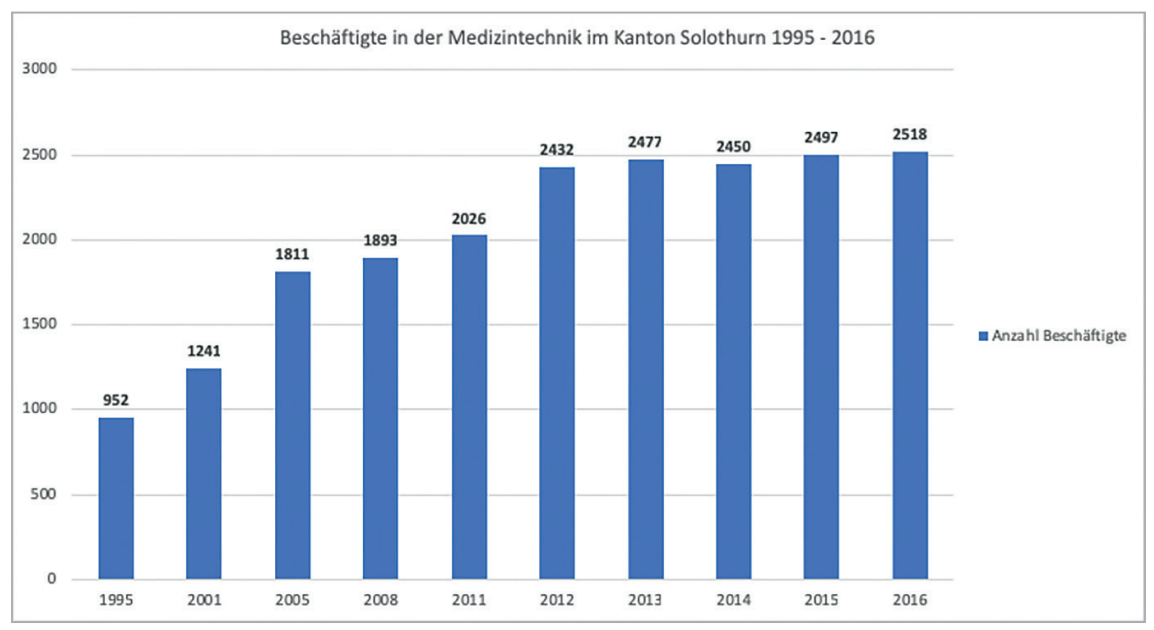

Quelle: Bundesamt für Statistik NOGA 1995-2008 und 2011-2016 


\section{Anhang 3: Beschäftigte Mathys AG*1955-2017}

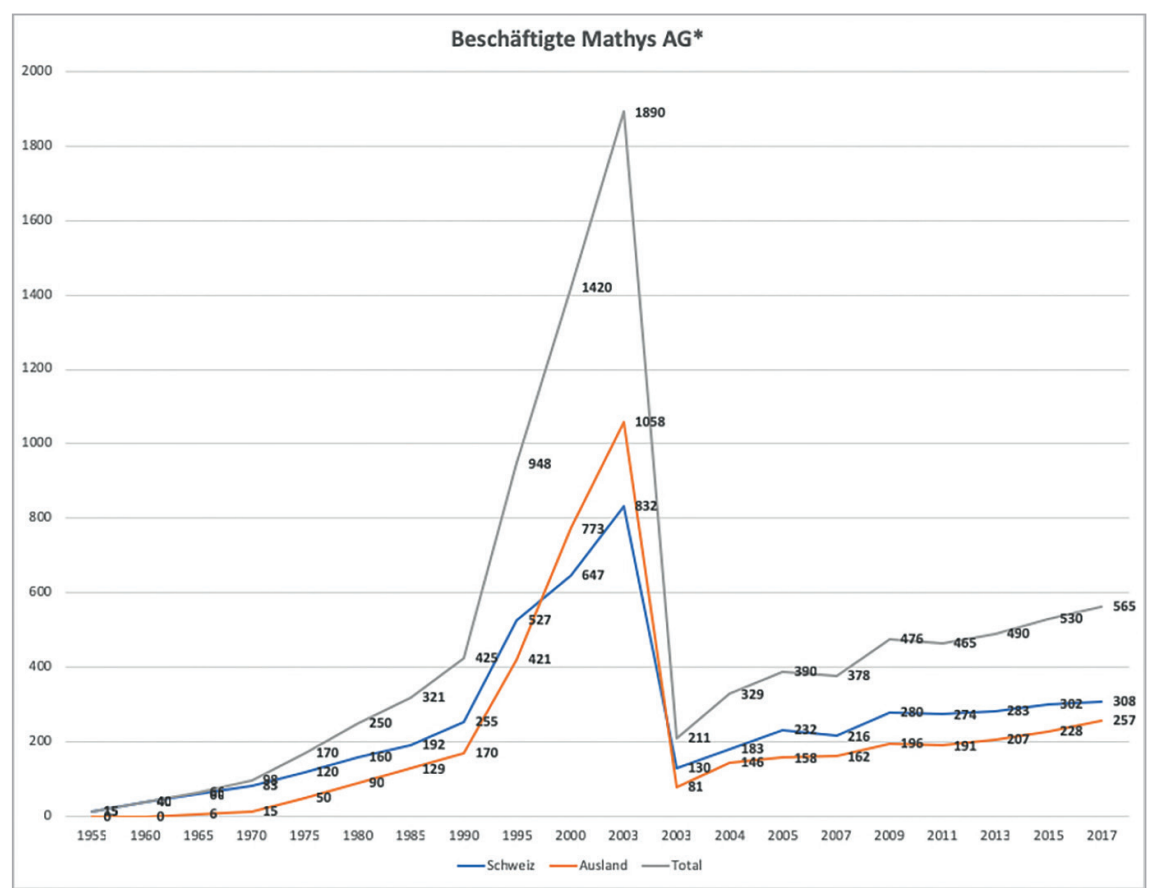

${ }^{*}$ Robert Mathys > Robert Mathys Co. > Mathys AG > Mathys Medizinaltechnik AG; ab 2003 Mathys AG (Orthopädie)

Quelle: Angaben von Robert Mathys jun. 


\section{Anhang 4: Beschäftigte RMS Foundation Bettlach*}

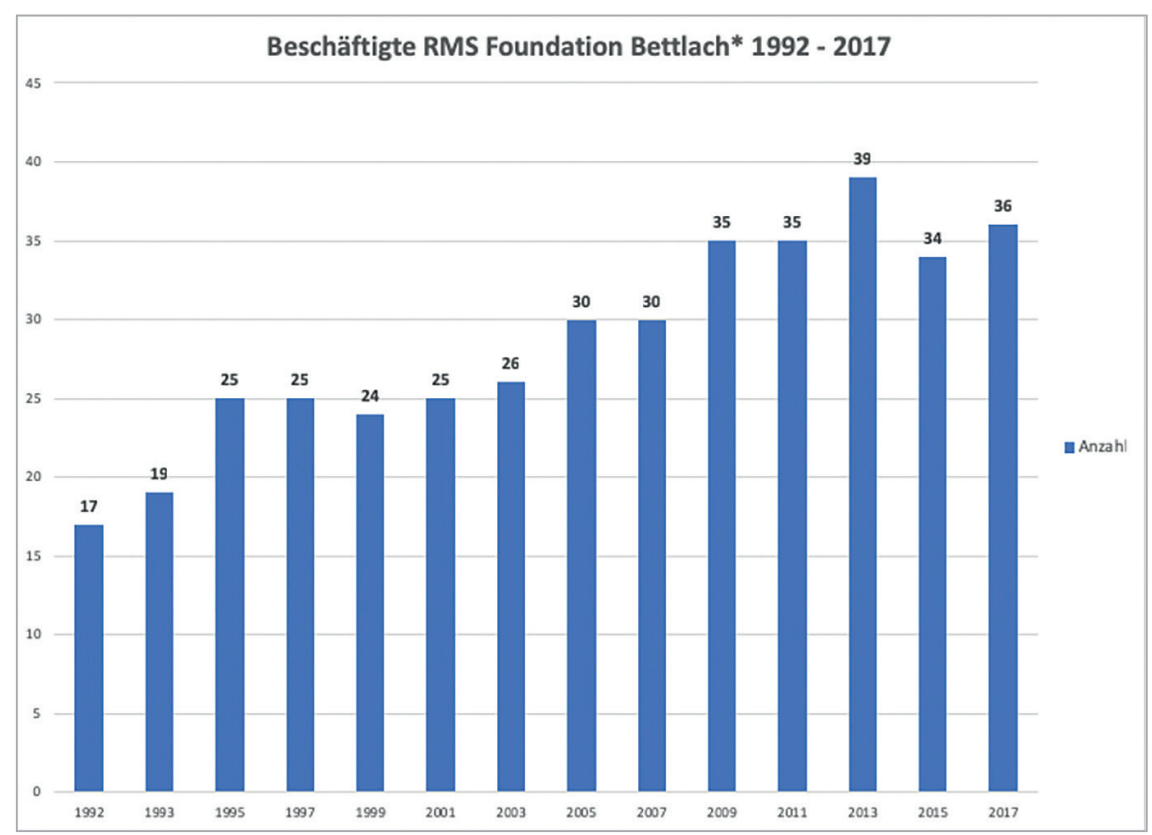

${ }^{*}$ Nach Ausgliederung aus Mathys Medizinaltechnik AG 1992 Quelle: Angaben von Robert Mathys jun. 


\section{Anhang 5: Beschäftigte Synthes* im Kanton Solothurn zwischen 2007 und 2018}

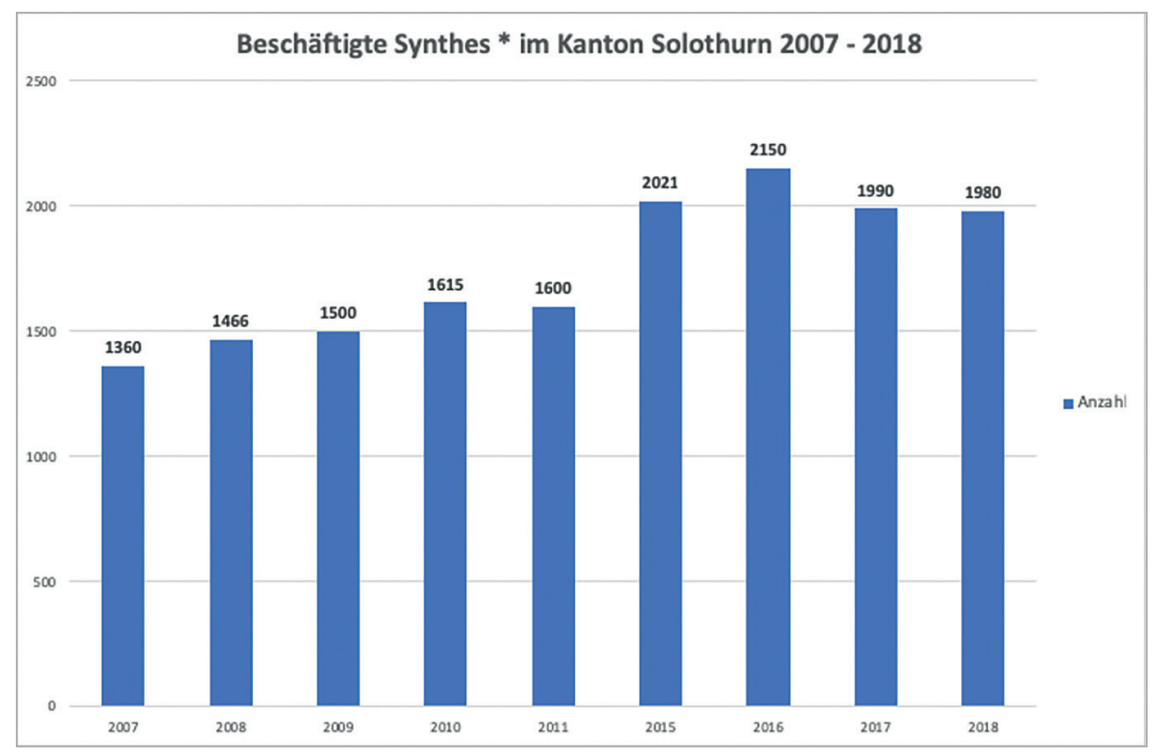

*2007-2012 Synthes, Inc.; ab 2012 De Puy Synthes

Die Zahlen sind in Vollzeitäquivalente umgerechnet. Keine Angaben für 2012, 2013 und 2014 Quelle: TOP 100, November 2007-November 2018 


\section{Anhang 6: Beschäftigte Stryker Selzach} zwischen 2007 und 2018

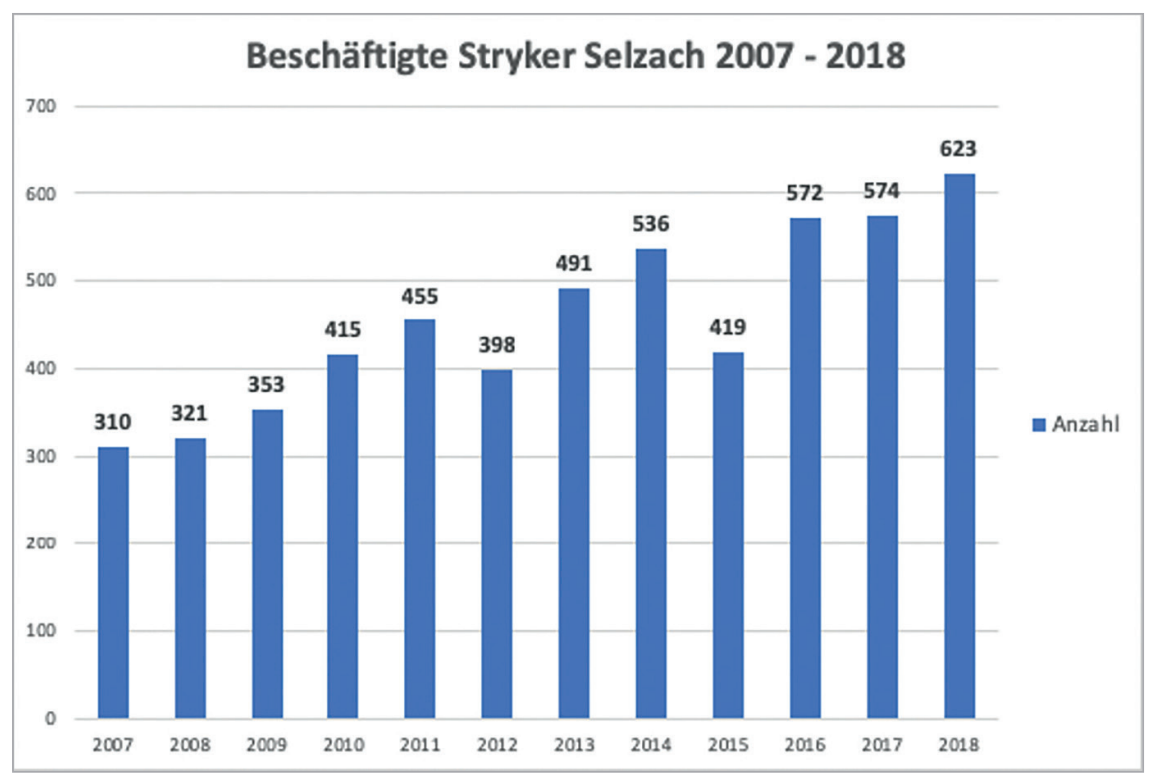

Die Zahlen sind in Vollzeitäquivalente umgerechnet.

Quelle: TOP 100, November 2007-November 2018 
Anhang 7: Beschäftigte Ypsomed Holding AG zwischen 2005 und 2018

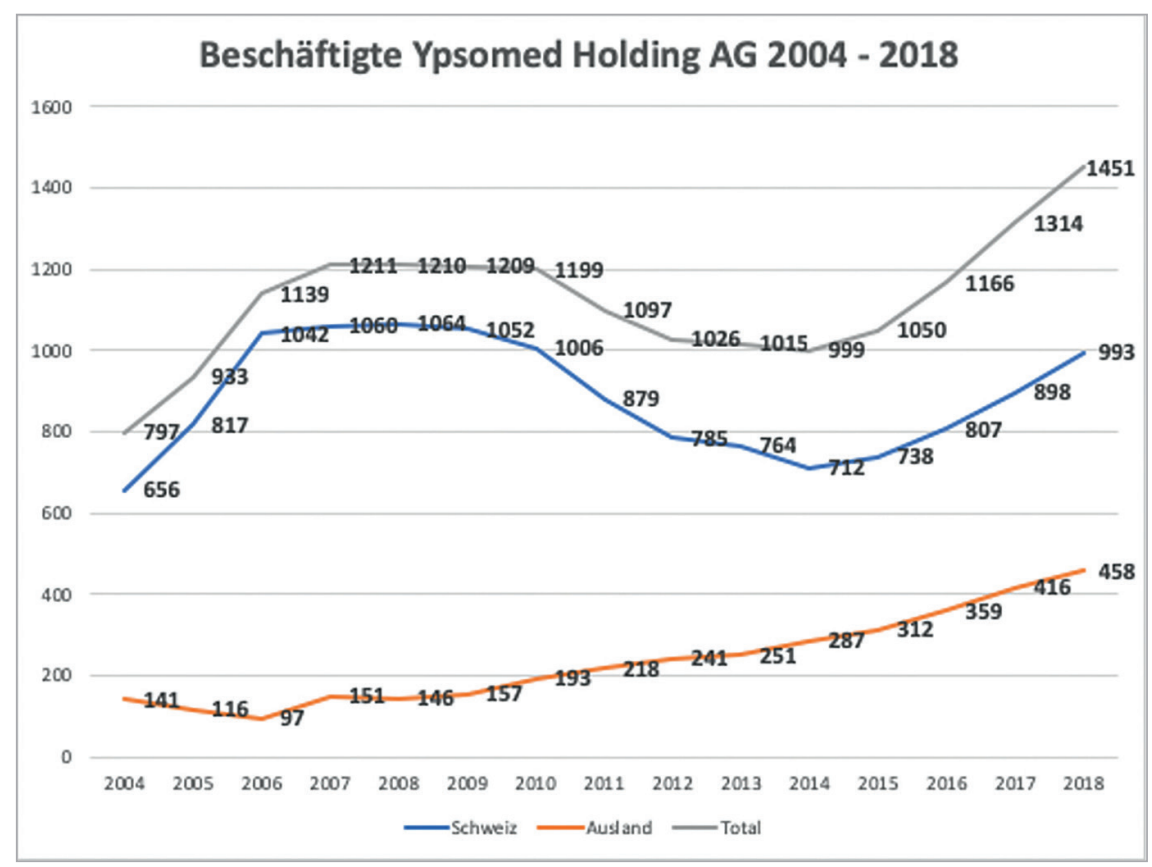

Quelle: Angaben von Michael Zaugg, Ypsomed Holding AG 


\section{Anhang 8: Beschäftigte Ypsomed Holding AG an den Standorten Burgdorf und Solothurn* 2012-2018**}

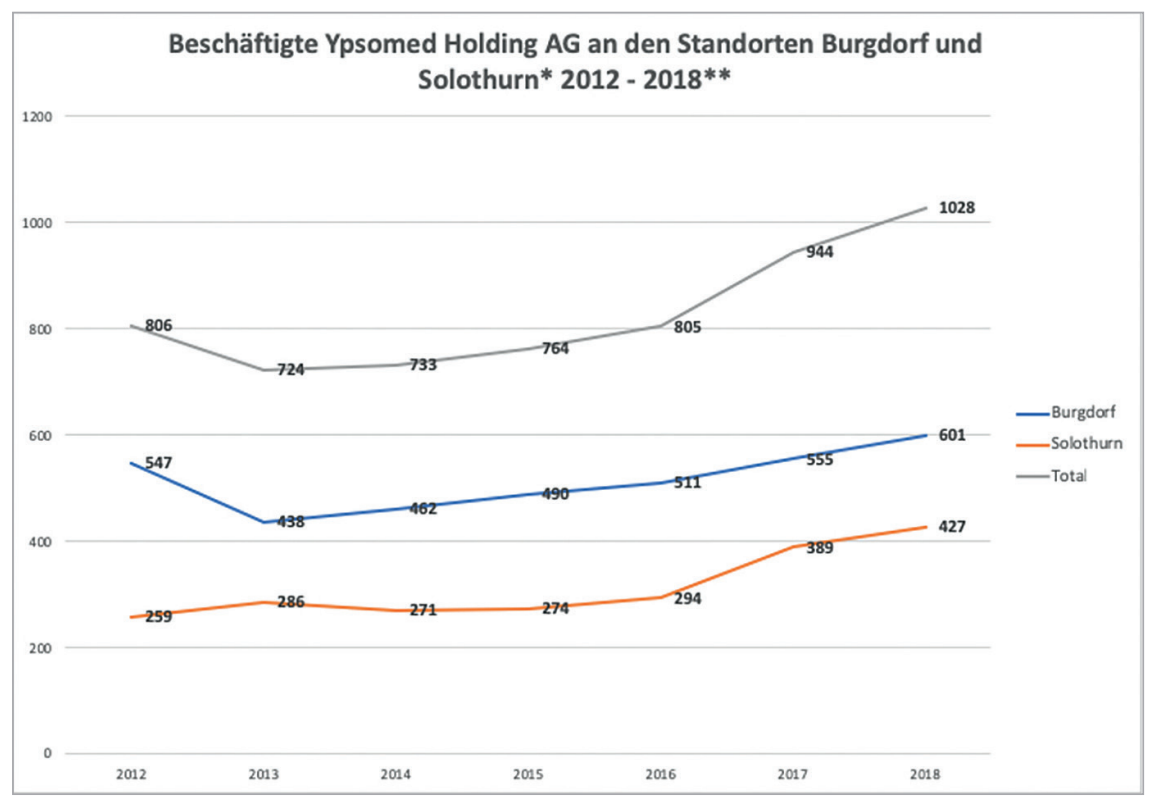

* inkl. Ypsotec Grenchen

** Eine Aufteilung in Burgdorf und Solothurn wird erst ab 2012 vorgenommen. Die Zahlen werden zu verschiedenen Terminen innerhalb eines Jahres erfasst. Der Gesamtwert für die Schweiz kann deshalb im Vergleich mit der vorangehenden Grafik leicht variieren.

Quelle: Angaben von Michael Zaugg, Ypsomed Holding AG 


\section{Bibliografie}

\subsection{Fachliteratur}

Berghoff, Hartmut / Sydow, Jörg: Unternehmerische Netzwerke - Theoretische Konzepte und historische Erfahrungen, in: Berghoff, Hartmut / Sydow, Jörg: Unternehmerische Netzwerke. Eine historische Organisationsform mit Zukunft?, Stuttgart 2007

Bergier, Jean-François: Die Wirtschaftsgeschichte der Schweiz. Von den Anfängen bis zur Gegenwart, Zürich, Köln 1983

Betschon, Franz / Betschon, Stefan / Lindecker, Jürg Dominik / Schlachter, Willy (Hrsg.): Ingenieure bauen die Schweiz, Technikgeschichte aus erster Hand, Band 1, Zürich 2013

Betschon, Franz / Betschon, Stefan / Lindecker, Jürg Dominik / Schlachter, Willy (Hrsg.): Ingenieure bauen die Schweiz, Technikgeschichte aus erster Hand, Band 2, Zürich 2014

Bibliothèque cantonale et universitaire, Fribourg (Hrsg.): Les Fribourgois sur la planète, Fribourg 1987

Bohlhalter, Bruno: Unruh. Die schweizerische Uhrenindustrie und ihre Krisen im 20. Jahrhundert, Zürich 2016

Bonjour, Edgar: Theodor Kocher, Bern 1981

Borner, Silvio / Porter, Michael E. / Weder, Rolf / Enright, Michael: Internationale Wettbewerbsvorteile: Ein strategisches Konzept für die Schweiz, Frankfurt a. M. 1991

Breiding, R. James / Schwarz, Gerhard: Wirtschaftswunder Schweiz. Ursprung und Zukunft eines Erfolgsmodells, Zürich 2016

Bundesamt für Statistik: Eidgenössische Betriebszählung 1985. Arbeitsstätten und Beschäftigte nach Wirtschaftsarten, Bern 1986

Bundesamt für Statistik: Die Kantone im Vergleich 1985/91/95, Bern 1998

Casson, Marc: Der Unternehmer. Versuch einer historisch-theoretischen Deutung, in: Geschichte und Gesellschaft. Zeitschrift für historische Sozialwissenschaft, Nr. 27, Göttingen 2001

Donzé, Pierre-Yves: Bâtir, Gérer, Soigner. Histoire des établissements hospitaliers de Suisse romande, Chêne Bourg-Genève 2003

Donzé, Pierre-Yves: Histoire de l'industrie horlogère suisse, Neuchâtel 2009

Dümmler, Patrick: Wissensbasierte Cluster in der Schweiz: Realität oder Fiktion? Das Beispiel der Medizinaltechnikbranche, Zürich 2005 (Dissertation an der ETH)

Eidgenössisches Statistisches Amt: 4. Eidgenössische Betriebszählung 25. August 1955, Band 4. Die Gewerbebetriebe in den Kantonen, Bern 1960 
Eidgenössisches Statistisches Amt: Eidgenössische Betriebszählung September 1965, Industrie und Gewerbe, Band 3. Betriebe, Hauptergebnisse nach Kantonen, Bern 1967

Eidgenössisches Statistisches Amt: Eidgenössische Betriebszählung 1975. Arbeitsstätten, Hauptergebnisse für die Kantone, Bern 1977

Etter, Richard / Hollenstein, Heinz: Die konjunkturelle Entwicklung der Solothurner Industrie im Zeitraum 1978-1985. Ein Vergleich mit der gesamtschweizerischen Entwicklung anhand des KOF/ETH-Konjunkturtests, Zürich 1986

Farago, Peter / Kriesi, Hanspeter (Hrsg.): Wirtschaftsverbände in der Schweiz. Organisation und Aktivitäten von Wirtschaftsverbänden in vier Sektoren der Industrie, Einleitung und Schlussfolgerungen, Grüsch 1986

FASMED: Medizintechnik - eine Schlüsselbranche für die Schweiz, Bern 2010

Finanzdepartement des Kantons Solothurn: Beiträge zur Statistik des Kantons Solothurn, Reihe W (Wirtschaftsstatistik). Heft 2. Betriebszählung Kanton Solothurn 1985 , Solothurn 1987

Frick, Andres / Hollenstein, Heinz: Innovationskraft und Innovationsverhalten in der Solothurner Wirtschaft, Zürich 1986

Hafner, Wolfgang: Die Krise und ihre Bewältigung. Jüngste Wirtschaftsgeschichte des Kantons Solothurn, Baden 2012

Halbeisen, Patrick / Müller, Margrit / Veyrassat, Béatrice (Hrsg.): Wirtschaftsgeschichte der Schweiz im 20. Jahrhundert, Basel 2012

Heim, Urs F. A.: Das Phänomen AO. Gründung und erste Jahre der Arbeitsgemeinschaft für das Studium der Osteosynthese, Bern 2001

Jahrbuch für Solothurnische Geschichte, Band 73 (2000)

Jeannet, Jean-Pierre: Leading a Surgical Revolution, Cham 2019

Kienzle, André: «Es gibt nur ein Gerlafingen!» Herrschaft, Kultur und soziale Integration in einer Standortgemeinde des Stahlkonzerns von Roll, 1918-1932, Zürich 1997

Lätt, Jean-Marie: 120 Jahre Arbeiterbewegung des Kantons Solothurn, Zürich 1990

Lipp, Silvan: Standort Schweiz im Umbruch. Etappen der Wirtschaftspolitik im Zeichen der Wettbewerbsfähigkeit, Zürich 2012

Mahrer, Stefanie: Handwerk der Moderne. Jüdische Uhrmacher und Uhrenunternehmer im Neuenburger Jura 1800-1914, Wien, Köln, Weimar 2012

Mumenthaler, M.: 75 Jahre M. Schaerer A.-G. Wabern-Bern. Kleine Chronik der Firma M. Schaerer A.-G. in Wabern-Bern, Fabrik für Arzt- und Spitalbedarf und Apparatebau 1882-1967, o. O. 1967

Murer Mecattaf, Laura / Frey, Jonas / Schmolders, Annebelle / Biedermann, Peter: Schweizer Medizintechnikindustrie (SMTI)-Branchenstudie, Bern 2016

RMS Foundation (Hrsg.): Engagement in zweiter Generation. Robert Mathys, Biel 2014

RMS Foundation (Hrsg.): Seminar zum 75. Geburtstag von Dr. h.c. Robert Mathys, Bettlach 1996

Rütter, Heinz / Nathani, Carsten / Popp, Jutta / Holzhey, Matthias: Wirtschaftliche Bedeutung der Medizintechnik in der Schweiz, Bern 2010 (im Auftrag von FASMED Dachverband der Schweizerischen Handels- und Industrievereinigungen der Medizinaltechnik)

Scheidegger, Urs: Solothurner Pioniergeist. Eine Reise durch die Solothurner Wirtschaftsgeschichte, Langendorf 2007 
Schlich, Thomas: Surgery, Science and Industry. A Revolution in Fracture Care, New York 2002

Schmitt-Rütt, Stephanie / Esslinger, A. Susanne / Schöffski, Oliver: Der Markt für Medizintechnik. Analyse und Entwicklungen im Wandel der Zeit, Burgdorf 2007

Strahm, Rudolf H.: Die Akademisierungsfalle. Warum nicht alle an die Uni müssen, Bern 2014

Wyss, Hedi: Hansjörg Wyss - mein Bruder, Wettingen 2014

Ypsotec (Hrsg.): 100 Jahre Ypsotec, o. O. 2016

\subsection{Quellen}

\section{Zeitungen}

Basellandschaftliche Zeitung, Ausgabe vom 29.2.2008

Basler Zeitung, Ausgabe vom 4.4.2002

Berner Zeitung, Ausgaben vom 19.5.2011, 17.5.2014, 24.5.2017

Cash, Ausgabe vom 15.7.2004

Der Bund, Ausgaben vom 14.11.2004, 16.11.2005, 23.4.2011, 27.5.2011

Finanz und Wirtschaft, Ausgaben vom 16.8.2003, 21.7.2007, 4.9.2010, 18.2.2012, 19.12.2012

Handelszeitung, Ausgaben vom 8.11.2000, 23.10.2002, 19.8.2003, 21.7.2007, 9.1.2014

Le Temps, Ausgabe vom 28.4.2011

Neue Zürcher Zeitung, Ausgaben vom 11.8.2000, 14.8.2003, 2.12.2003 (Sonderbeilage Medizinaltechnik in der Schweiz), 7.5.2006, 20.2.2008, 28.5.2008, 28.4.2011, 11.6.2O11, 13.2.2012, 14.6.2O12, 4.4.2016, 14.7.2017, 5.11.2017, 24.5.2018, 20.7.2O18, 6.9 .2018

NZZ Folio; Ausgabe 11/2005

Solothurner Zeitung, Ausgaben vom 6.7.1981, 31.1.1996, 24.6.2009, 23.6.2013, 8.8.2016, 2.9.2016, 19.11.2017, 8.6.2018, 19.7.2018, 13.9.2018, 5.10.2018

Tages-Anzeiger, Ausgabe vom 21.5.2018

\section{Zeitschriften}

AO Dialogue 14, Davos 2001

Crédit Suisse (Hrsg.): Investment, Solutions \& Products: Regionalstudie Wirtschaftsraum Jurasüdfuss. Swiss Issues Regionen. Jurasüdfuss: Life Sciences sorgen für Aufwind, Bern Mai 2016

Die Volkswirtschaft. Das Magazin für Wirtschaftspolitik, Ausgabe 1/2, Bern 2010

Gesnerus, Schweizerische Zeitschrift für Medizin und Naturwissenschaften, Vol. 66.2, Basel 2009

Gesnerus, Schweizerische Zeitschrift für Medizin und Naturwissenschaften, Vol.70.1, Basel 2013 
Handelskammer Deutschland - Schweiz, Handelskammerjournal 7.6.2016

SO Economy, herausgegeben von az Solothurner Zeitung, in Zusammenarbeit mit

Baloise Bank SOBA und Kanton Solothurn Wirtschaftsförderung, 16.6.2011

TOP 100. Eine Sonderbeilage von Solothurner Woche, Solothurner Tagblatt,

Ausgaben: November 2007, November 2008, November 2009, November 2010,

November 2011, November 2012, November 2013, November 2014, November 2015 , November 2016, November 2017, November 2018

Traverse: Zeitschrift für Geschichte, Band 19 (2012), Heft 2, Pflegekrisen

\section{Geschäftsberichte}

Swatch Group: Geschäftsbericht 2018, Biel 2019

Synthes: Geschäftsbericht 2005, Geschäftsbericht 2007, Geschäftsbericht 2008,

Geschäftsbericht 2009, Annual Report Synthes 2010

Synthes-Stratec: Jahresbericht 1999, Geschäftsbericht 2000, Geschäftsbericht 2001, Geschäftsbericht 2002

Ypsomed Holding AG: Geschäftsbericht 2004/05, Geschäftsbericht 2005/06, Geschäftsbericht 2006/07, Geschäftsbericht 2010/11, Geschäftsbericht 2014/15, Geschäftsbericht 2017/18

\section{Präsentationen}

Bundesamt für Statistik: NOGA 2011-2016, unter https://www.pxweb.bfs.admin.ch/pxweb/ de/px-x-0602010000_103/px-x-0602010000_103/px-x-0602010000_103.px, abgerufen am 10.2.2019

Bundesamt für Statistik: NOGA 1995-2008, unter https://www.bfs.admin.ch/bfs/de/home/ statistiken/kataloge-datenbanken/daten.assetdetail.290096.html, abgerufen am 10.2.2019

Eschbach, Lukas: Medtech im Kanton Solothurn, unter: www.standortsolothurn > Wirtschaftsstandort $>$ MedTech $>$ Medtech im Kanton Solothurn, abgerufen am 12.1.2O19

Graber, Roger, Wirtschaftsförderung Kanton Solothurn: MedTech-Landschaft Kanton Solothurn, Community Event MedTech Solothurn, 20.9.2017, Solothurn 2017, erhältlich: Kanton Solothurn Wirtschaftsförderung, Roger Graber 03262794 51 oder http://www.standortsolothurn.ch/fileadmin/standortsolothurn/pdf/ wirtschaftsstandort/MedTech_Community_Anlass_2017.pdf, abgerufen am 12.1.2019

Graber, Roger / Müller, Philippe: Life-Science-Landschaft Kanton Solothurn, Solothurn 2018 (Präsentation am Community Event vom 29.8.2018 in Bettlach), erhältlich: Kanton Solothurn Wirtschaftsförderung, Roger Graber 0326279451

Wirtschaftsförderung Kanton Solothurn: Solothurner Life Science-Industrie 2018. 4. Solothurner MedTech-Cluster, Solothurn 2018 (Präsentation am 
Community Event vom 29.8.2018 in Bettlach), erhältlich: Kanton Solothurn Wirtschaftsförderung, Roger Graber 0326279451 oder www.standortsolothurn.ch $>$ Wirtschaftsstandort $>$ Medtech $>$ Medtech-Hotspot der Schweiz $>$ Life-Science. Industrie 2018, abgerufen am 31.3.2019

\section{Firmendarstellungen}

Julius Bär (Hrsg.): Swiss Research. Gesellschaftsstudie Synthes-Stratec Inc., Zürich 22.3.1999 Blum, Stefan: Synthes-Stratec, Sarasin Basic Report, o. O. Oktober 1999 De Puy Synthes: Integrität in Aktion - Health Care Compliance, o. O. August 2012 Furler, Markus: Stratec, Sarasin-Studie, o. O. April 1996

Gachnang, Peter, Bank Julius Bär und Co AG: Unternehmerportraits - Medizinaltechnik (Branchenstudie), Zürich 16.9.1999

greaterzuricharea.com > Life Sciences $>$ Erfolgsgeschichten $>$ stryker, abgerufen am 11.1.2019

Helvea: Synthes. Nailing Success, Genf 2006

Mathys AG Bettlach: Ganzheitliche Medizinaltechnik, o. O. 1991 (Firmenbroschüre)

Pictet: Synthes-Stratec Healthcare / Switzerland, Genf 3.6.2002

Robert Mathys: Es gibt auch humanitäre Industrie in der Schweiz, o. O. 1968/69

(Erscheinungsjahr gemäss Auskunft von Robert Mathys jun.)

Robert Mathys: Robert Mathys stellt für die ganze Welt Produkte für die Knochenchirurgie her, die den Menschen die Freude am Leben zurückgeben, o. O. 1971/72

(Erscheinungsjahr gemäss Auskunft von Robert Mathys jun.)

Sonova Website: www.sonova.com > Über uns > Fakten und Zahlen, abgerufen am 31.3.2019 Stratec Holding AG: Verkaufsprospekt für Börsengang, o. O. 1996 Stratec Medical: Im Dienste der Gesundheit, o. O., nicht datiert

Straumann-Website: www.straumann.com > Über uns > Zahlen und Fakten, abgerufen am 31.3.2019

Straumann-Website: www.straumann.com > Über uns > Unsere Geschichte, abgerufen am 31.3.2019

Stryker: http://www.stryker.de/de/standorte/selzach/, abgerufen am 31.3.2019

Synthes: Synthes. Dedicated to Health, o. O., nicht datiert

Synthes: Synthes in der Schweiz. Wo alles begann, o. O., nicht datiert 


\section{Persönliche Gespräche}

Bruno Bohlhalter, Autor der Dissertation Unruh. Die schweizerische Uhrenindustrie und ihre Krisen im 20. Jahrhundert, Zürich 2016, Oekingen, 21.12.2017

Jesus Fernandez, Regionalverantwortlicher UNIA Biel Seeland/Solothurn, Biel, 14.8.2018

Robert Frigg, Geschäftsführer 41 medical AG, Bettlach (ehemals engster Mitarbeiter von

Hansjörg Wyss, Synthes), Bettlach, 23.4.2018

Urs Graber, Projektleiter, Wirtschaftsförderung des Kantons Solothurn, Solothurn, 7.3.2018

Wolfgang Hafner, Wirtschaftshistoriker, Brugg, 5.3.2018

Robert Mathys jun., Stiftungsratspräsident Robert Mathys Stiftung / RMS Foundation, Bettlach, 20.12.2017 und 7.3.2018

Simon Michel, CEO Ypsomed Holding AG Burgdorf und Solothurn, Solothurn, 18.6.2018

Thomas Straumann, Gründer und Verwaltungsrat der Straumann Group Basel sowie

Gründer und Verwaltungsratspräsident von Medartis Basel, Basel, 12.8.2020

Thomas Wahl, ehem. Generalmanager Stryker Selzach, Telefongespräch am 6.6.2018

\subsection{Gesprächsprotokolle}

\section{Robert Mathys jun.}

Robert Mathys jun., Stiftungsratspräsident RMS Foundation, während vieler Jahre Mitglied und Präsident des Verwaltungsrates sowie Leiter F \& E der Mathys AG Bettlach.

Das Gespräch fand am 20. Dezember 2017 in Bettlach statt.

Wie kam es zur ersten Begegnung zwischen dem Chirurgen Maurice E. Müller und ihrem Vater, Robert Mathys?

Als Bieler hatte Maurice E. Müller Kontakt zum Stahllieferanten Notz, der meinen Vater mit rostfreiem Material belieferte. So wurde er auf meinen Vater aufmerksam. Zuvor hatte Müller schweizweit Kontakt mit mehreren Chirurgieinstrumentemachern. Diese waren jedoch nicht bereit, einen gewissen Vorlauf zu finanzieren. Müller wollte eine Entwicklung technologisch zur Reife bringen, danach die Anwender schulen und schliesslich das Produkt auf dem Markt platzieren.

Diese Vorgehensweise stand am Ursprung der kurz darauf gegründeten Arbeitsgemeinschaft für Osteosynthese (AO). Die Risiken der operativen Knochenchirurgie waren den Medizinern durchaus bewusst (z. B. defekte, ungenügende Befestigungsmittel). Sie wollten vor dem Markteintritt ein fertiges, sicheres Produkt. Demgegenüber wollten die Firmen, die Müller vor meinem Vater kontaktiert hatte, das Produkt gleich sofort verkaufen und auf eine Vorfinanzierung verzichten. Diese Vorlaufphase betrug schon damals zwei bis vier Jahre. 
Was stellte Ihr Vater vor dem Umstieg auf die Medizintechnik her?

Er war spezialisiert auf allgemeine Befestigungsmittel wie Schrauben und Muttern aus rostfreiem und säurebeständigem Stahl. Dieses Know-how war damals noch kein Allgemeingut. Die Bearbeitung von rostfreien Stählen benötigte bestimmte Anforderungen, da diese relativ «zähhart» waren. Notz empfahl also Müller «den von Bettlach».

Die Firma meines Vaters war ein Décolletage-Betrieb, der schon zwischen 1946 und 1958 eigene Produkte entwickelt hatte. Mit seinen ungefähr fünfzehn Mitarbeitern war er Zulieferer für grosse Firmen von Befestigungssystemen. Er produzierte beispielsweise Hähne und Fittings für die Milchindustrie. Hingegen verzichtete er auf den Einsatz von Messing, weil bereits die gesamte Uhrenindustrie damit arbeitete.

Nach dem ersten Kontakt mit Maurice E. Müller sagte mein Vater: «Das ist meine Chance, da gehe ich jetzt einfach rein.» Er war überzeugt, Eigenes entwickeln und neue technologische Inputs geben zu können.

Ihr Vater hatte offenbar das Gespür für Marktlücken.

Das stimmt. Zudem war die Chemie zwischen den beiden von Anfang an so gut, dass Müller meinen Vater begeistern konnte. In der Regel bestand zu dieser Zeit eine strikte Trennung zwischen Ärzten und «normalem Volk». Der einfache Arbeiter wurde kaum anerkannt, die Standesunterschiede waren ausgeprägt. Es war überhaupt nicht selbstverständlich, dass ein Mann wie mein Vater freien Zugang zu der bereits bestehenden Gruppe um Maurice Müller hatte. Mein Vater nahm die Impulse auf und gab alles, um die Entwicklung erfolgreich zu gestalten. Zwischen April 1958 und November/Dezember 1958 hatte er bereits die ersten Grundinstrumentarien in Kastenform entwickelt. Als Selfmademan hatte er sich die notwendige Technologie angeeignet. Nach seiner Mechanikerlehre hatte er sich ständig weitergebildet.

Aufgrund der vielen Inputs hat Müller meinen Vater kennen und schätzen gelernt. Dieser zeigte immer wieder auf, wie man etwas vernünftig herstellen kann. Das Verhältnis mit Müller wurde danach freundlich-familiär.

Markiert die Beziehung zwischen Müller und Ihrem Vater generell den Beginn der Zusammenarbeit zwischen Chirurgen und Produzenten?

Ja, am Ursprung stand das gegenseitige Verständnis der beiden. Sie haben sich trotz verschiedener Fachsprachen gefunden. Müller hatte ein technologisches Vorstellungsvermögen, und mein Vater konnte später auch Operationen beiwohnen, etwa im Spital Grenchen bei Dr. Guggenbühl, der zum erweiterten Kreis der AO gehörte. Dass er danach als erster Nichtakademiker einen AO-Kurs besuchen durfte, machte Furore. Der Dialog wurde immer besser, auch wenn Technologie und Medizin ursprünglich verschiedene Welten waren.

Wesentlich war die systematische Organisation der Instrumente und des Materials in Kästen. Schraubenkasten und Plattenkiste wurden zu einem ganzheitlichen Instrumentarium in kompletten Sets zusammengefügt. Um die erforderliche Systematik der Hilfsmittel zu erreichen, musste der Produzent die Arbeitsabläufe in der Chirurgie genau kennen. Die von meinem Vater erstellten Sets waren wiederum die Basis für die Schulung der Ärzte und des Operationspersonals sowie für das AO-Manual, das diese Technologie beschrieb.

Parallel zur Traumatologie entwickelte Müller in seiner Funktion als Orthopäde Hüftprothesen, nachdem er zuvor spezielle Hüftplatten hergestellt hatte. Im Gegensatz zur Traumatologie wollte Müller diesen Bereich für sich behalten. 
Wie konnte Ihr Vater den Wechsel zur Medizintechnik wirtschaftlich verkraften?

Tatsächlich fokussierte er den Betrieb ab 1958 fast ausschliesslich auf die Medizintechnik. Das hat ihm finanziell beinahe den Kopf gekostet, weil er - wie erwähnt - mit dem Markteintritt zuwarten musste. Auf Initiative von Müller gewährten die Banken vor Ort meinem Vater einen gewissen Spielraum. Das wäre bei heutigen Startups nicht mehr möglich. Ohne fixfertigen Businessplan geben die Banken keine Kredite mehr.

Eine weitere Schwierigkeit bestand darin, dass damals die Uhrenindustrie vor Ort praktisch alle Fachleute kontrollierte. Qualifizierte Décolleteure konnten nicht einfach in andere Branchen abwandern. Mein Vater musste diese selber anlernen. Die umfassende Dominanz der Uhrenindustrie ist heute kaum mehr vorstellbar.

Wo sehen Sie die Berührungspunkte zwischen Medizintechnik und Uhrenindustrie?

Gemeinsam ist das Gefühl für Präzision. Im Gegensatz zur Uhrenindustrie geht es allerdings in der Medizintechnik nicht um 1/10oo Millimeter. Zudem sind die Materialien sehr unterschiedlich. Die Uhrenindustrie arbeitet mit Messing. Dieses Material ist leicht zu verarbeiten, die Abnutzung der Werkzeuge ist deshalb minimal. Demgegenüber arbeiten wir mit rostfreiem Stahl, Titan, Kobalt-Chrom-Legierungen - alles harte, zähe Stoffe mit hohem Werkzeugverschleiss.

Hingegen haben Uhrenindustrie und Medizintechnik dasselbe Verständnis von Décolletage (früher benötigte man dafür Exzenterscheiben). Heute ist die Uhrenindustrie allerdings weitgehend automatisiert und auf Kleinteilefabrikation spezialisiert. Da braucht es keine Décolleteure mehr. Die Maschinen, einmal eingestellt, produzieren Zehntausende von Kleinteilen.

Kurz zusammengefasst: Die Technologie ist in beiden Branchen dieselbe. Die Uhrenindustrie ist aber filigraner. Neben den Grössenordnungen sind auch die Materialien und Werkzeuge unterschiedlich.

Wie entwickelte sich die Firma Mathys nach dem Einstieg in die Medizintechnik weiter?

Sie verbreitete sich rasch über ihre eignen Grenzen hinaus. Aufgrund der Organisation und der Grösse der Firma hat man schon bald eingesehen, dass man nicht alles allein machen konnte. Deshalb arbeitete Mathys mit anderen Instrumenteherstellern oder Décolletage-Betrieben (wie Rieger in Grenchen) zusammen. Wir hatten stets ungefähr ein Drittel unserer Produktion durch Zulieferung, damit wir notfalls ausweichen konnten. Je nach spezifischen Bedürfnissen hatten wir verschiedene Zulieferer.

Mit anderen Personen gründete mein Vater neue Zulieferfirmen, da wir am Standort Grenchen keine Fachleute mehr hatten und daher auslagern mussten.

Vereinzelt sprangen auch Leute von uns ab. So gründeten die ehemaligen Mitarbeiter Vogt und Karpf 1963 die Firma VOKA, die ein Jahr später in Osteo umbenannt wurde. In unserem Nachbardorf Selzach entstand ein neuer Konkurrent. Dieser wiederum wurde 1996 vom amerikanischen Konzern Stryker übernommen.

Bereits 1960 trat das Forschungsinstitut Straumann aus Waldenburg (BL) auf den Plan.

Am Ursprung standen Materialprobleme. Die AO erhoffte sich von Straumann rostfreie Stähle von besserer Qualität. Folglich wurde er beigezogen. Etwas später stieg er auch in die Produktion ein.

Zunächst entstand aus dieser Situation ein ziemlicher Knatsch. Die Berner Seite der AO mit Maurice E. Müller unterstützte Mathys, während die Basler mit Allgöwer und Willenegger das Gefühl hatten, man könne Mathys übergehen, weil man mit Strau- 
mann besser bedient sei. Nachdem sich mein Vater gewehrt hatte, teilten Straumann und Mathys die Märkte untereinander auf. Daraufhin wurden mit der Synthes AG in Chur Lizenzverträge abgeschlossen. Das war die Basis für Produktion und Preisbildung. Später kam Synthes USA als weiterer Partner hinzu. Nach dessen Fusion mit Stratec Medical hatte die Synthes AG mit Synthes-Stratec und Mathys erneut zwei Ansprechpartner. Diese Kooperation wurde Ende der 1990er-Jahre von der europäischen Kartellgesetzgebung als unzulässige Absprache kritisiert. Diese heiklen Rahmenbedingungen waren mit ein Grund dafür, dass Mathys 2004 die Traumatologie an die nachmalige Synthes Inc. verkaufte, welche nunmehr zum alleinigen Partner der Synthes AG Chur (AO) wurde.

Wie beurteilen Sie die aktuelle Situation der Medizintechnik am Jurasüdfuss?

Bezüglich der Beschäftigung lässt sich die Medizintechnik nicht genau quantifizieren. Statistisch läuft sie unter der Metallindustrie. Auch die Zulieferer lassen sich nicht spezifisch erfassen, weil sie ja auch noch für andere Branchen produzieren.

Unbestritten ist jedoch die Bedeutung der Innovation. Engineering hat in der Medizintechnik nach wie vor einen hohen Stellenwert, auch wenn die Produkteentwicklung heute durch die Kostenentwicklung im Gesundheitswesen gebremst wird. Der Spardruck führte in den letzten Jahren dazu, dass bestimmte Technologien gar nicht mehr auf den Markt gekommen sind. So beträgt etwa die Produkteeinführung eines Implantates sechs bis zehn Jahre, was den Entwicklungsprozess derart verteuert, dass das Gesamtsystem die daraus entstehenden Kosten nicht mehr zu decken vermag. Hinzu kommt, dass in der Traumatologie die marktgängigen Produkte ziemlich ausgereift sind. Auch in der Prothetik ist der Innovationsdruck nicht mehr sehr gross. Heute erhält man den Investitionsaufwand nicht mehr zurück, sodass bei De Puy Synthes und auch bei uns Forschung und Entwicklung (F\&E) eher rückläufig sind, zumal die Technologie ein sehr hohes Niveau erreicht hat.

Die Tendenz geht in Richtung "günstiger produzieren». Ich war früher immer Optimist, heute sehe ich für den Werkplatz Schweiz Probleme kommen. Wir haben unseren Vorsprung eingebüsst. Wer die Automatisierung nicht stark vorantreiben und - wie in der Medizintechnik - keine Riesenserien produzieren kann, verlagert die Produktion ins Ausland. Einfache Produkte sind schon heute "Made in China».

Was bleibt, ist F \& E. In diesem Bereich sehen wir bei der Medizintechnik ähnliche Konzentrationsprozesse wie in anderen Branchen. Kleine Firmen werden von den grossen übernommen, die wiederum gezielte Entwicklungsaufträge nach aussen an spezialisierte Betriebe geben. Dieses Phänomen kennen wir schon seit längerem in der Pharmaindustrie.

Ist die RMS Foundation nicht auch auf Forschung spezialisiert?

Die RMS Foundation wurde 1985 von meinem Vater nach dem Vorbild der AO gegründet. Wir wollten für die Schulung der Ärzte und für die Überwachung der technologischen Entwicklung ein quasi neutrales Instrumentarium. Fünf Jahre später haben wir die Firma Mathys in die Divisionen "Trauma», "Künstlicher Gelenkersatz» und "Oralimplantologie» unterteilt. Als Forschungseinheit für Labor und Tests haben wir die RMS aus der Produkteentwicklung herausgelöst und zum neutralen Dienstleister für die drei Hauptbereiche aufgebaut. Seit 1993 ist die RMS rechtlich und finanziell unabhängig. Der Fokus der Forschung liegt auf Material- und Fertigungstechnologie. Das Schulungswesen wird von der Firma selber für die Ärzte stark produkteorientiert durchgeführt. 
Ab 1992 wurde die RMS auch Dienstleister für andere Firmen. Mit Mathys haben wir bestimmte Projekte, die wir aus Konkurrenzgründen anderen Betrieben nicht anbieten. Heute sind Mathys und De Puy Synthes unsere wichtigsten Kunden. Sie und andere Medtech-Firmen beziehen Leistungen gegen Bezahlung. Wir liefern unseren Kunden Beratung und Informationen, die sie für die Registrierung ihrer Produkte benötigen. Dazu gehören Testleistungen nach Normen.

Sie haben die Unterteilung von Mathys in drei Tätigkeitsfelder erwähnt.

Seit 1960 kooperierte Müller mit Mathys nicht nur in der Traumatologie, sondern auch in der Entwicklung von Prothesen. Diesen Bereich bearbeitete Müller selbstständig und klammerte ihn folgerichtig von der AO aus. Er gründete dafür die Firma Protek, die er später an Sulzer verkaufte. Als Zulieferer von Protek und danach von Sulzer war Mathys zuständig für bestimmte Prothesensegmente. 1996 machte sich Mathys im Bereich des künstlichen Gelenkersatzes selbstständig.

Ab 1975 entwickelte mein Vater zudem Implantate für die Oralchirurgie. Daraus entstand 2001 die Firma Thommen Medical in Grenchen.

Wie waren bzw. sind Ihre Beziehungen zu Behörden und Sozialpartnern?

Wir waren und sind ein typischer Familienbetrieb, in dem die Mitarbeiter stets einen hohen Stellenwert hatten. Wir hatten immer gut qualifizierte Leute und sind - im Gegensatz zu amerikanischen Firmen - sozial eingestellt. Das wird uns auch von ehemaligen Mitarbeitern bestätigt. Während Grossfirmen die Entscheide weit weg vom Arbeitsplatz treffen, bleiben wir überschaubar. Selbst als wir 1700 Mitarbeiter hatten, haben wir den Kontakt zu diesen hochgehalten. Heute haben wir etwas mehr als 500, davon 300 in Bettlach. Wir sind weiterhin eine Familiengesellschaft, in der der persönliche Kontakt unvermindert wichtig ist.

Unsere Familie blieb immer bescheiden. Ohne grosse Exzesse. Diese Glaubwürdigkeit ist wichtig.

Wie sind die Zukunftsaussichten der Medizintechnik in der Schweiz?

Nach einer turbulenten Entwicklungszeit wird die Medizintechnik eine gewisse Konsolidierung erfahren. Ich hoffe, dass die Schweiz den Lead in F \& E und Technologie behalten kann. Wenn aber die Fertigung wegbricht, werden auch die medizintechnischen Dienstleister Dämpfer einstecken.

Die Medizintechnik ist und bleibt eine Zukunftstechnologie, doch müssen wir aufpassen, dass uns der Zug nicht abfährt. Es sei bloss darauf hingewiesen, welche Summen Deutschland, unterstützt vom Staat, in F \& E investiert. Ich befürchte, dass die Schweiz die in den letzten vierzig bis fünfzig Jahren aufgebaute Führungsposition einbüssen wird.

Hinzu kommt, dass die Eintrittsbarriere in den Markt der Medizintechnik infolge der Regulierungsflut enorm hoch ist. Seit ein paar Jahren reguliert in Europa die Medical Device Regulation (MDR) alles. Dadurch erhöht sich - wie bereits erwähnt - die Markteinführung eines Produktes beträchtlich. Die Eigenverantwortung der Medtech-Produzenten nimmt kontinuierlich ab. Darunter leiden namentlich die KMU. 


\section{Robert Frigg}

Robert Frigg war als Chief Technology Officer (Forschung und Entwicklung) bei Synthes Inc. engster Mitarbeiter von Hansjörg Wyss und ist heute Geschäftsführer der 41medical AG, Bettlach. Er ist Ehrendoktor der Universitäten Zürich und Salzburg und Gastprofessor am Berlin Institute of Health der Charité Universitätsmedizin Berlin.

Das Gespräch fand am 23. April 2018 in Bettlach statt.

\section{Wie kamen Sie zur Medizintechnik?}

Mich interessierte die Fliegerei. Für die Ausbildung zum Piloten fehlte das Geld. Folglich habe ich mich für die Option Flugzeugmechaniker entschieden. Dieser Weg führte über eine Ausbildung zum Mechaniker und danach über eine Zusatzausbildung als Flugzeugmechaniker. Als ich 1978 die vierjährige Lehre abgeschlossen hatte, wurden die Weiterbildungsangebote zum Flugzeugmechaniker abgeschafft. Ich stand vor dem Nichts.

Ich wollte deshalb eine Zweitlehre als Fotograf starten und reagierte auf ein entsprechendes Angebot der Arbeitsgemeinschaft für Osteosynthese (AO) Davos. Der Leiter des AO-Forschungsinstitutes, Stephan Perren, fragte mich gleich bei meinem Einstieg, ob ich für zwei Monate in der mechanischen Werkstätte aushelfen könne, um einen Abgang zu überbrücken. Ich war einverstanden und stellte für Ingenieure Prototypen her, die jedoch teilweise grottenschlecht waren. So brachte ich dann selber erste Ideen ein, worauf mich der Abteilungsleiter von einer Weiterarbeit in der Mechanik zu überzeugen versuchte. Nach einigem Zögern - ich hatte immer noch die Fotografenlaufbahn vor Augen - willigte ich ein. Die folgenden fünf Jahre war ich in allen Abteilungen der AO tätig: in der Histologie, der Biomechanik, bei Testaufbauten und der Assistenz an Operationen und als Tierpfleger.

Welches waren die nächsten Karriereschritte?

Nach der Aktivität in den verschiedenen Abteilungen wurde ich als jüngstes Mitglied in die Technische Kommission (TK) der AO aufgenommen. Da war die ganze AO-Führungsriege mit Müller, Schneider, Allgöwer, Willenegger, Perren, um nur einige zu nennen. Die Struktur war damals noch sehr hierarchisch, ich war gleichsam als Zuhörer geduldet («Hören Sie zu, da können Sie lernen»). Immerhin hatte ich die Chance, Neues auszuprobieren, und habe auf dieser Basis erste Patente angemeldet.

Entscheidend für meine Weiterentwicklung innerhalb der AO waren zwei Grundfähigkeiten, über die ich bis heute verfüge. Ich sehe rasch, ob ein Produkt oder eine Vorgehensweise gut ist oder nicht. Dasselbe gilt, wenn ich jemandem zuschaue, der ein Instrument in den Händen hält. Mein Aufstieg wurde auch dadurch begünstigt, dass ich hierarchisch niemanden bedrohte. Die Chirurgen, insbesondere aus den USA und Deutschland, baten mich bei ihren Schulungen um Hilfe.

Höchstens Robert Mathys und sein Mitarbeiter Toni Cotting, beides ebenfalls Mechaniker und Konstrukteure, betrachteten mich zunächst als Konkurrenten. Nachdem wir uns jedoch auf der Sachebene gefunden hatten, entstand eine tiefe Freundschaft.

Sie haben später ja bei Mathys gearbeitet.

Nach einem Vorgesetztenwechsel bei der AO habe ich 1997 gekündigt, weil mir die Wertschätzung fehlte. Ich arbeitete dann für Mathys und Synthes USA. Bei Mathys hörte ich oft: «Du bist zu teuer.» Meine Antwort: «Eine normale Schraube kann jeder herstellen, 
eine qualitative Neuentwicklung kostet jedoch Geld.» Tatsächlich habe ich für Platten eine neue Verschraubungstechnik erfunden. Nach dieser Erfindung verzehnfachte sich bei Mathys die Nachfrage nach Platten.

Was braucht es neben dem Fachwissen, um einen derartigen Erfolg zu erreichen?

Entscheidend ist der richtige Umgang mit Chirurgen. Diese haben immer recht ... Folglich musste ich sie dazu bringen, dass sie meine Neuerungen als die ihren betrachteten. Das erfordert Diplomatie. Wohl auch deshalb wollten die Chirurgen nur noch mit mir zusammenarbeiten. Auf diesem Weg entstanden - über die berufliche Ebene hinaus enge Beziehungen.

Früher war man regelmässig bei Operationen dabei, leistete Support. Techniker und Entwickler waren in den Kliniken zuhause. Hansjörg Wyss, der Handwerk, Können und Wissen schätzte, erkannte die Bedeutung der Kooperation aller wichtigen Akteure. Das war sein Mantra, das setzte er konsequent um. Heute besteht diese Beziehung zwischen Chirurgen und Produzenten nicht mehr - ein massiver Rückschritt. So dürfen in mehreren Staaten der USA Aussendienstmitarbeiter bei Operationen nicht mehr anwesend sein. Genau das war aber ein wichtiger Schlüssel zum Erfolg.

Nach der Übernahme von Synthes durch Johnson \& Johnson (J \& J) wurde mein enges Netzwerk anerkennend gewürdigt, doch musste ich aus Gründen der Compliance meine Kontakte zu den Medizinern abbrechen bzw. auf ein Minimum reduzieren. Generell werden bei Grossfirmen die Kontakte zwischen Wissenschaft und Produktion wesentlich beschränkt. Gespräche werden nur ausnahmsweise bewilligt. Sie erfordern ellenlange Rechtfertigungen. Es müssen ausführliche Notizen über Relevanz und Nutzen der Gespräche erstellt werden. Unter solchen Bedingungen werden Innovationen beinahe verunmöglicht. Aus diesen Gründen habe ich bei J\& J gekündigt. Es ging dort nicht mehr um Produkteentwicklung, sondern um Verkaufsstrategien. Das hat mich nicht mehr interessiert.

Sie haben ja auch verschiedene Fusionen erlebt.

Von 1997 bis 2004 arbeitete ich für Mathys und Synthes USA. Nach der Übernahme von Mathys durch Synthes übersiedelte ich für zwei Jahre in die USA. 2011 wurde Synthes von J\& J übernommen. Bei all diesen Fusionen gingen wichtige Netzwerke für immer verloren. Früher flog Fritz Straumann umgehend nach Helsinki, wenn ein finnischer Chirurg Probleme hatte. Wenn mich ein Mediziner aus Berlin anrief, war ich anderntags dort. Heute kennt man sich nicht einmal mehr.

War (und ist) nicht gerade die Kooperation zwischen Chirurgen einerseits, Herstellern und Entwicklern andererseits ein zentraler Erfolgsfaktor in der Medizintechnik?

Am intensivsten war die Zusammenarbeit innerhalb der AO. Dort hatten die Mediziner den Lead. Bei Innovationen wurden diese systematisch beigezogen. Förderlich war dafür auch der hierarchiefreie Umgang unter den Ärzten selbst. Auch wenn durchaus eine gewisse Konkurrenz bestand, etwa zwischen der "Müller-Schule» und der "Allgöwer-Schule», war die Grundstimmung freundschaftlich, kooperativ. Dies war mit ein Grund für die jahrelangen zweistelligen Gewinne bei Synthes, die allerdings bereits vor der Übernahme durch J \& J zurückgingen.

Das ganze Umfeld hat sich geändert. Bei den Grossfirmen werden die Chirurgen heute nur noch punktuell kontaktiert. Auch in den Klinken selbst ist deren Freiraum kleiner geworden. Der Einkauf bestimmt, welche Produkte angeschafft werden, die Chirurgen sind zu blossen Angestellten geworden. 
Wie reagieren Sie selbst auf diese Veränderungen?

Nach dem Weggang bei J\&J hat mich Hansjörg Wyss in die von ihm übernommene Firma Startec GmbH in Bettlach geholt, mit der Absicht, den Medtech-Zweig aufzubauen und massiv auszuweiten. 2016 wurde die Firma in 41medical umbenannt. Dazu ist festzustellen, dass Wyss und ich bis in die jüngste Zeit während vierzig Jahren eng zusammengearbeitet haben. Ich habe von Wyss sehr viel gelernt. Er ist enorm grosszügig und ebenso hartnäckig, wenn er etwas durchsetzen will. Für mich war er ein extremer Glücksfall. Wyss und ich waren geradezu ein Dream-Team; er liess mir freie Hand, auch beim Aufbau von 41medical.

Als Verwaltungsratspräsident dieses KMU-Unternehmens habe ich die Kontakte zu den Chirurgen Schritt für Schritt wieder reaktiviert. Wir sind zertifiziert, um Medtech-Produkte zu entwickeln, herzustellen und zu vertreiben. Mittlerweile nimmt die Medizintechnik 95 Prozent des Umsatzes ein. 41medical hat jedoch keine eigenen Produkte auf dem Markt. Wir sind Zulieferer für ungefähr dreissig Kunden (auch J \& J), mit drei Schwerpunkten. 1) Die Kunden bezahlen uns für unsere Erfindungen eine Lizenzgebühr. Sie lagern also die Risiken gewissermassen aus und übernehmen eine Neuentwicklung erst, wenn diese rentiert. 2) Wir übernehmen Auftragsentwicklungen. Das ist der grösste Bereich. 3) Wir unterstützen Start-ups, indem wir ihnen Qualitätssysteme verkaufen, mit denen sie ihre Ideen bis in die Kliniken hineinbringen können. Diese Funktion nehmen wir bei der ETH, beim Campus Balgrist und auch durch meine Gastprofessur an der Berliner Universitätsklinik Charité wahr. Insbesondere die beiden erstgenannten Aktivitäten erfordern sehr hohe Vorinvestitionen.

Was fasziniert Sie an der Medizintechnik?

Speziell sind die Prozessabläufe zwischen Patienten, Chirurgen, Unfall, Nachbehandlung. In der daraus entstehenden Dynamik ist das Produkt nur ein Teil. Es kann nicht isoliert betrachtet werden. Die Kernfrage ist für mich, wie ich mit dem Produkt Bestehendes optimieren kann. Beeinflussung der anderen Akteure durch Visualisierung und Schulung hat einen hohen Stellenwert. Das Erfinden neuer Produkte und Vorgehensweisen lässt mich nicht los. Ich bleibe beharrlich bis zur Lösung. In meinem Berufsleben habe ich 250 Patente entwickelt.

Oft wird die Verwandtschaft der Medizintechnik mit der Uhrenindustrie betont. Wie sehen Sie die Beziehungen zwischen diesen beiden Branchen?

Uhrenindustrie und Medizintechnik sind zwei Paar Schuhe. Sowohl die Materialien als auch der Grad der Präzision sind unterschiedlich. Während in der Medizintechnik rostfreier Stahl und Titan benutzt werden, bestehen die "Innereien" einer Uhr aus Messing. Auch in der Décolletage sind die Prozesse unterschiedlich. In der Uhrenindustrie werden um ein Vielfaches grössere Serien produziert. Im Technopark von Sulzer wollten wir die Zusammenarbeit mit Uhrenfachleuten vertiefen, doch hatten wir schlicht zu wenig Gemeinsamkeiten. Die Verwandtschaft zwischen den beiden Industriezweigen ist also stark zu relativieren.

Gemeinsam ist generell das Qualitätsbewusstsein. Das habe ich bei meiner Mechanikerlehre im Bündnerland nie in dem Ausmass erlebt, wie es etwa am Jurasüdfuss der Fall ist. 
Warum ist der Jurasüdfuss zu einem weltweiten Hotspot der Medizintechnik geworden? Und wie beurteilen Sie die Zukunftsaussichten?

Am Ursprung stand Robert Mathys. Dann gab es Ableger wie etwa die von ehemaligen Mathys-Ingenieuren gegründete Osteo, welche 1996 von Stryker übernommen wurde. Weil Mathys nicht alles selber produzieren konnte, nahm er Kontakt mit Zulieferern auf bzw. beteiligte sich selber an der Gründung neuer Zulieferbetriebe. Die breite gewerblich-industrielle Abstützung, die daraus hervorging, ist heute Gold wert.

Ich befürchte hingegen, dass die grossen Firmen wegziehen, wenn die Unternehmenssteuerreform nicht bald realisiert wird. Zudem sind die Zulassungen in den USA einfacher. Hinzu kommt, dass wir eine gewisse Marktsättigung haben. Grösster Hemmschuh ist jedoch die extreme Regulierungsdichte in Europa, mit der Medical Device Regulation (MDR). Diese wird in einem Jahr mit einer Übergangsfrist von drei Jahren in Kraft treten. Gemäss MDR muss man im Voraus beweisen, dass eine Innovation besser ist als bestehende Angebote. Das ist praktisch unmöglich. An den strikten Neuregulierungen werden viele Zulieferer zerbrechen. Fachleute rechnen damit, dass in Europa ein Drittel aller Medtech-Betriebe schliessen wird.

Wie äussern sich die europäischen Neuregulierungen konkret?

Wenn ich beispielsweise eine neue Schraube entwickle, wird in Zukunft verlangt, dass ich mein Dossier mit dem Dossier des andern Produktes genau vergleichen muss. Konkret: Ich muss meinem Konkurrenten mitteilen, dass ich eine neue Schraube entwickeln werde. Weiter muss ich eine klinische Studie machen, um zu beweisen, dass meine neue Schraube besser ist als die bisherige. Das ist blosse Theorie, weil die Qualität der Schraube vom Chirurgen, vom Patienten und dessen Knochenbeschaffenheit abhängig ist. Der ganze Qualifizierungs- und Zertifizierungsprozess hängt in der Luft. Das ist weiter auch nicht verwunderlich, weil die Firmen in der Regel die schlechtesten Mitarbeiter in die Normenvereinigung schicken. Dort werden dann unrealisierbare Vorschriften zusammengeschustert.

Als Folge davon werden einfache Entwicklungen blockiert, weil der finanzielle Aufwand zu gross ist. Grössere Projekte werden extrem teuer und komplex. Auch bei der Materialentwicklung bremsen die neuen EU-Regulierungen. Die zu entwickelnde Menge ist verhältnismässig gering, sodass die dafür notwendigen Investitionskosten gescheut werden.

Wo steht heute die AO?

Sie ist nach wie vor das grösste Chirurgennetzwerk der Welt. Früher war sie aktiv in der Entwicklung, die Industrie durfte ein Produkt erst dann verkaufen, wenn sie grünes Licht gab. Heute engagiert sich die AO zu 90 Prozent in der Schulung. F\&E sind marginal, sie beschränken sich bloss auf Grundlagenforschung, während früher die Produkteentwicklung im Vordergrund stand. Primär werden Prinzipien geschult. Aber am Schluss sind eben doch das Instrument und die Zusammensetzung der Werkkästen entscheidend. So umfasst ein Wirbelsäulen-Set drei Boxen mit 200 Instrumenten. Selbst Aussendienstmitarbeiter werden nicht mehr konkret geschult.

Eine ähnliche Entwicklung lässt sich am Jurasüdfuss bei J \& J beobachten. Solothurn ist zwar immer noch zentraler Standort für Europa, auch die Anzahl Fachleute dürfte ungefähr gleich geblieben sein. Diese befassen sich aber nicht mehr mit F \& E, sondern mit der Dokumentation von Produkten. 


\section{Simon Michel}

Simon Michel ist CEO der Ypsomed Holding AG Burgdorf und Solothurn.

Das Gespräch fand am 18. Juni 2018 in Solothurn statt.

Wie entstand die Ypsomed als Produzent von Insulinpens und -pumpen?

Mein Vater war ursprünglich im Aussendienst als Insulinverkäufer für die Firma Novo Nordisk tätig. Es fiel ihm auf, das ungenau gespritzt wurde. Zusammen mit seinem Bruder entschloss er sich deshalb, ein Gerät herzustellen, das Insulin automatisch verabreichte. 1984 gründeten sie zusammen in Burgdorf die Disetronic Holding AG. Ein Jahr später hatten sie die erste Insulinpumpe entwickelt, die 1987 auf den Markt kam. Weil die Pumpen nicht immer funktionierten, entwickelten sie parallel dazu Insulinpens, die bei Ausfall der Pumpe eingesetzt werden konnten. Das Pumpen- und Pengeschäft wuchs kontinuierlich, der Markt dehnte sich über Europa und die USA aus.

Trotzdem waren wir zu klein, um mit der Konkurrenz mitzuhalten. Als die gleichzeitig entstehende Minimed 2002 an Medtronic verkauft wurde, hatten wir, verglichen mit diesem Grossunternehmen, drei Mal weniger Leute im Aussendienst.

Wie hat Disetronic auf diese Situation reagiert?

2003 verkauften wir Disetronic für 1,6 Mrd. CHF an Roche. Gleichzeitig haben wir für ungefähr 500 Mio. CHF das Pengeschäft von Roche zurückgekauft und Ende des Jahres neu als Ypsomed mit drei Standorten in Burgdorf firmiert.

Um die Jahrtausendwende zeigte sich, dass Pens immer wichtiger werden würden. Medikamente wurden seltener oral eingenommen und immer häufiger subkutan gespritzt, weil deren Moleküle zu gross waren, um vom Magen verarbeitet werden zu können.

Ende 2003 begann der Ausbau von Ypsomed nach Solothurn. Was waren die Gründe?

Nachdem der französische Chemiekonzern Sanofi mit dem Insulin-Medikament Lantus die Penbestellungen sprunghaft erhöht hatte, mussten wir unsere Kapazität innert kurzer Zeit massiv erweitern. Dafür benötigten wir mehr Fläche. Die drei Burgdorfer Standorte waren für eine handarbeitsintensive, nicht aber für eine automatische Produktion mit einer 35 Meter langen Maschine geeignet.

Relativ rasch konnten wir in Solothurn grosse Hallen erwerben, da Flextronics, ein Produzent von Kunststoffspritzguss, den dort neu aufgebauten Standort nach kurzer Zeit wieder auflösen wollte. (Die Hallen hatte er von der Ascom erworben.) Zwischen 2003 und 2005 konnten wir schrittweise das ganze Firmengelände übernehmen. Wir haben in Solothurn unsere Spritzguss-Aktivitäten kontinuierlich ausgebaut.

2007/o8 ging die Nachfrage von Sanofi stark zurück, weil der französische Chemiegigant auch andere Penhersteller berücksichtigte. Wie haben Sie diesen Rückschlag überwunden?

Es war Schock und Chance zugleich. Wir mussten abbauen, erhielten aber gleichzeitig die Chance, Pens für andere Abnehmer zu produzieren. Heute sind wir absoluter Weltmarktleader im Entwickeln und Herstellen von Pens. Wir sind de facto Monopolist.

$\mathrm{Ab} 2006$ begannen wir, die Aktivitäten vom Projekt- ins Produktionsgeschäft zu verschieben. Weltweit führten wir Gespräche mit über 150 Pharma- und Biotechfirmen. Wir entwickelten Plattformen für Pens und antizipierten den Bedarf der Firmen. Von der Plattform zum Produkt verkürzten wir die Zeit von vier Jahren auf vier Monate. 
Die Pharmafirmen produzieren nicht mehr selber, müssen aber für das Produkt mehr bezahlen. Auf diesem Weg generieren wir eine Ebit-Marge von fast 35 Prozent. Im Gegensatz zu uns schuf die Konkurrenz keine Plattformen. Unser Vorteil: Wir stellen auf derselben Automationsschiene Produkte für verschiedene Kunden her - Produkte, die nicht identisch, aber ähnlich sind. Durch den Wechsel vom Projekt- zum Produktgeschäft ist eine Standorterweiterung aktuell geworden.

Vor bald zehn Jahren sind Sie ins Insulinpumpengeschäft zurückgekehrt. Was waren die Gründe für diesen Schritt?

Bei dem Verkauf an Roche hatten wir beim Pumpengeschäft einen Marktanteil von 70 Prozent. Dieser fiel allmählich auf 15 Prozent. Diese Tatsache sowie die Diversifizierung im Pengeschäft motivierten uns 2009 zur Rückkehr in diesen für uns traditionellen Bereich. Zwischen 2011 und 2018 haben wir zwanzig Tochtergesellschaften aufgebaut, deren Logistik entwickelt und 1000 Personen ausgebildet, die den Umgang mit Pumpen schulen. Zudem verfügen wir über virtuelle Callcenter in siebzehn Sprachen. Im Gegensatz zu den Pens, die von uns an andere Firmen geliefert werden (Business to Business, B2B), liefern wir die Pumpen an die Verbraucher (Business to Consumer, B2C). Diese werden also direkt via Arzt an die Patienten verkauft. Bei den beiden Vorgehensweisen sind Entwicklung und Herstellung identisch, unterschiedlich ist die Distribution. Bei B2B ist unser Name nicht sichtbar, bei B2C steht «Ypsomed» drauf.

Zurzeit wächst das Pumpengeschäft mit über 20 Prozent, das Pengeschäft mit gut 10 Prozent. Unser Umsatz beträgt 466 Mio. CHF bei einem Ebit von 13 Prozent. Bis in drei Jahren peilen wir einen Umsatz von über 500 Mio. CHF und einen Ebit von 20 Prozent an. Dieses Ziel ist realistisch, weil wir uns in Wachstumsmärkten bewegen und über die Plattformen ein Alleinstellungsmerkmal haben. Wir sind heute Kosten- und Technologieführer; Kostenführer dank vieler Kunden und daher grosser Anlagen, Technologieführer, weil wir das ausreichende Geld für Investitionen in neue Produkte/Plattformen haben (2017 waren es 9 Prozent des Umsatzes).

Wie sehen Sie die Entwicklungsmöglichkeiten im Standort Solothurn?

An unserem Standort Solothurn war mit Flextronics bereits zuvor eine Firma im Kunststoffspritzguss tätig. Wir konnten auf bestehendes Know-how zurückgreifen und haben fast 150 Personen von Flextronics übernommen. Dies ist umso wichtiger, als die entsprechende Technologie primär auf Erfahrung basiert. Unsere Kunststoffspritzgiessmaschinen der Netstal-Maschinen AG waren zudem weitgehend identisch mit jenen von Flextronics. Diese Rahmenbedingungen waren ein Glücksfall für uns, die Arbeitnehmer und den Kanton.

Seither haben wir die Infrastruktur stark erneuert, indem wir unsere verschiedenen Produktionen hoch automatisiert haben. Aus Gründen der Kapazitätsauslastung arbeiten wir 24/7, im 4- bzw. 5-Schichtbetrieb. Die fünfte Schicht umfasst über das Wochenende zweimal zwölf Stunden. Mit den Zulagen ergibt sich daraus ein Lohn von 100 Prozent.

Wie gestaltet sich die Zusammenarbeit mit den Behörden?

Die Zusammenarbeit mit dem Amt für Wirtschaft und Arbeit (AWA) Solothurn ist gut und weniger schwerfällig als im Kanton Bern. Demgegenüber ist Bern in der Wirtschaftsförderung agiler, etwa beim Unterstützen von Ansiedlungen.

Das AWA Solothurn ist für uns ein verlässlicher Partner. Ich erwähne beispielsweise 
die Hilfe bei der Neugestaltung der Schichtarbeit. Da zeigte sich, dass Solothurn eine grosse Tradition in Schichtarbeit hat.

Können Sie uns die Qualifikationsstruktur bei den Ypsomed-Arbeitnehmern in Solothurn genauer erläutern?

Auf dem Platz Solothurn haben wir ungefähr 350 Mitarbeiter, davon 250 in der Produktion. Davon wiederum sind rund 150 angelernt. Diese stammen oft aus anderen Berufsfeldern wie Bäcker, Logistiker, Automatiker, sind also fremdberuflich. Der Maschinenbediener muss nicht unbedingt Kunststofftechnologe sein, wesentlich wichtiger ist die praktische Erfahrung. Viele Mitarbeiter sind bei uns über zehn Jahre tätig. Teilweise haben wir sie von der Flextronics übernommen.

Vor der Anstellung klären wir jeweils innerhalb eines Tages ab, ob der Bewerber technisch begabt ist. Insgesamt verfügen ungefähr 60 Prozent von ihnen über eine Berufslehre, 40 Prozent haben keine berufliche Ausbildung. Deshalb bieten wir seit drei Jahren Nachholbildungen nach Art. 32 Berufsbildungsgesetz an, und zwar in den Berufen Produktionsmechaniker und Logistiker. Zurzeit machen zwölf Personen im Alter von 45 bis 55 Jahren von diesem Angebot Gebrauch.

Im Laufe der Jahre haben sich mit der Automatisierung Berufsinhalte und Qualifikationsanforderungen verändert. Der Mensch nimmt seine Funktionen heute vorwiegend in der Steuerung und Planung der Prozesse wahr. Wer früher ölige Hände hatte, analysiert heute Daten. Aus diesem Grunde gehen wir heute bei der Rekrutierung anders vor als vor zehn bis fünfzehn Jahren. Wir stellen praktisch keine Ungelernten ein.

Als wir in Solothurn einstiegen, brauchten wir vorwiegend arbeitswillige Maschinenbediener. Dabei haben wir - bei einem hohen Ausländeranteil - eine gute Arbeitsmentalität vorgefunden. Noch heute sind über 50 Prozent der Belegschaft in Solothurn Ausländer (gegenüber ungefähr 10 Prozent in Burgdorf). Diese vorwiegend aus dem Balkan stammenden Mitarbeiter sind bestens integriert, verfügen über solide Deutschkenntnisse und sind ausgesprochen betriebstreu. Das gilt übrigens für alle Arbeitnehmer der Ypsomed, beträgt doch die Fluktuationsrate in Solothurn weniger als 5 Prozent.

Der Jurasüdfuss ist eine Medtech-Region. Wie stark wirkt sich das auf die Ypsomed aus?

Generell hat Solothurn eine grössere Dichte an technisch-industriellen Mitarbeitern als der Kanton Bern. Trotzdem, eine «Medtech-Branche» als solche gibt es nicht. Bei uns arbeiten Kunststoffspritzer, bei Mathys oder Stryker Metallfräser oder -dreher. Dessen ungeachtet haben Feinmechanik und Uhrenindustrie hier Spuren hinterlassen. Die Leute sind präzises Arbeiten gewohnt und haben ein entsprechendes Mindset.

Wir profitieren insofern von der Medtech-Industrie, als wir immer Bedarf an ausgebildeten Fachkräften haben. In der Orthopädie hat - nicht zuletzt infolge der verstärkten Regulierung - eine Konsolidierung stattgefunden. Kleinere Firmen können teils nicht mehr mithalten, grössere verlieren Lieferanten. Gerade für Décolleteure bietet Ypsomed eine Alternative. Sie können im Umbau und in der Wartung von Maschinen und Kunststoffspritzen eingesetzt werden. So arbeiten bei uns in Burgdorf fünfzig, in Solothurn zwanzig Décolleteure.

Selber haben wir die Anzahl Lehrlinge von dreissig auf sechzig und die Anzahl Lehrberufe von sechs auf zwölf erhöht. Zudem fördern wir den Besuch der Fachhochschule, indem wir für den Absolventen bei einer Präsenz von 60 Prozent und einem Lohn von 70 Prozent die Schulkosten vollumfänglich übernehmen. Dieses Angebot wird ergänzt durch unsere Ypsomed Academy für Weiter- und Nachfolgebildung. 
Wie stark ist die zur Ypsomed gehörende Firma Ypsotec in die Medizintechnik eingebunden? Unsere Pens hatten vor 20 bis 25 Jahren viele Metallteile, die wir zum Teil von der damaligen Décolletage AG Grenchen erwarben. Nachdem diese verkauft wurde und einige Jahre später geschlossen werden sollte, übernahmen wir die Firma 1994 zu günstigen Bedingungen und tauften sie 2003 als Tochtergesellschaft der Ypsomed zu Ypsotec um.

Ypsotec hat sich vor ungefähr zehn Jahren umorientieren müssen, da die Pens praktisch keine Metallteile mehr aufweisen. Heute beliefert sie zu 50 Prozent die Orthopädie wie J \& J, Stryker und fast zur Hälfte andere Industriefirmen. Mit uns macht sie noch 5-6 Prozent Umsatz. Diese Diversifizierung weisen in der Region viele KMU auf.

Allgemein tendieren grosse Orthopädiefirmen dazu, weniger selber zu entwickeln und vermehrt bei KMU einzukaufen, weil eingekaufte Entwicklungen die Erfolgsrechnung nicht belasten, sondern in die Bilanz übergehen. Sie konzentrieren sich auf die Produktion und die Optimierung von Prozessen. Dieser Trend ist am Jurasüdfuss besonders ausgeprägt.

Kann am Jurasüdfuss von einem Medtech-Cluster gesprochen werden?

Nein, die Medizintechnik ist, wie erwähnt, zu diversifiziert. Wenn überhaupt, können wir eher von der Präzisionsmechanik als Cluster sprechen. Die Region besitzt dank der genau und sauber arbeitenden Fachkräfte eine gewisse Anziehungskraft. Das zeigt sich nun beispielsweise bei der Ansiedlung von Biogen in Luterbach, die aus dem Medtech-Gebiet Leute rekrutiert.

Von einem Medtech-Cluster kann allenfalls für die vergleichsweise immer noch begrenzte Region Bern-Biel-Zürich mit der schweizerischen Dachorganisation Swiss Medtech gesprochen werden. Abgesehen davon fehlt am Jurasüdfuss auch eine aktive Clusterpolitik mit regelmässigen Anlässen und Best Practice.

Die Zukunft der Medtech hängt in unserer Region wesentlich von einer vernünftigen Unternehmenssteuerreform ab. Sollten die Steuersätze für internationale Holdings zu hoch werden, besteht die Riesengefahr, dass sie ihren Sitz im Kanton Solothurn aufgeben werden.

Wie sind Ihre Beziehungen zu den Gewerkschaften?

Die Gewerkschaften sind in der Schweiz unbedeutend. Wir nehmen sie nicht wahr, da wir eng mit der internen Personalkommission zusammenarbeiten. Diese trifft sich alle sechs bis acht Wochen mit der Geschäftsleitung.

In einer sozialen Firma, bei der 74 Prozent der Aktien der Familie gehören, sind Gewerkschaften und Gesamtarbeitsvertrag (GAV) überflüssig; abgesehen davon, dass der GAV als Vertragskonstrukt ein Auslaufmodell ist. Unser Basislohn liegt heute weit über den vertraglichen Mindestlöhnen, da wir unsere Mitarbeiter mit guten Arbeitsbedingungen bei uns behalten wollen. Entscheidend ist für uns deren Motivation. Das schweizerische Arbeitsrecht kennt einen relativ schwachen Kündigungsschutz. Deshalb kommen aber auch viele Firmen, die kontinuierlich neue Arbeitsplätze schaffen, in die Schweiz.

Bei einem Familienbetrieb ist das Verantwortungsbewusstsein ohnehin höher als bei einem managergetriebenen Konzern, dessen Sitz womöglich in den USA ist. Als Familienfirma haben wir ein Grundcommitment, auch gegenüber der Öffentlichkeit. 


\section{Thomas Wahl}

Thomas Wahl war bis Ende Juni 2018 Generalmanager von Stryker Selzach. Heute ist er Inhaber einer Beratungsfirma im Bereich der Medizintechnik.

Das Gespräch fand telefonisch am 6. Juni 2018 statt.

Der amerikanische Konzern Stryker hat 1996 die Solothurner Medtech-Firma Osteo übernommen. Seither ist das Unternehmen aus Selzach massiv gewachsen. Was war das Motiv für die Übernahme?

Osteo wurde 1963 von den beiden ehemaligen Straumann-Ingenieuren Vogt und Karpf gegründet, zunächst unter dem Namen Voka. Das Unternehmen war auf Knochenbruchbehandlung und Gelenkersatz spezialisiert. Die Produkte waren analog, aber konzeptionell etwas verschieden vom Synthes-System.

In Zusammenarbeit mit Heinz Mittelmeier, Ordinarius für Orthopädie in Homburg, entwickelte Osteo 1974 ein System von Hüftprothesen, bei denen Hüftkopf und -pfanne aus Keramik gefertigt waren. Vor der Übernahme durch Stryker gehörte Osteo zum Medtech-Konzern Smith \& Nephew und hatte folgende Schwerpunkte: Knochenersatzmaterialien, Hüft- und Gelenkersatz (z.B. Schulter, Finger, Fussgelenk) und Knochenbruchbehandlung (Traumatologie). Zwischen 1986 und 1996 richtete die Firma den Fokus auf Gelenkersatz und Weiterentwicklung der Marknagelung.

Stryker übernahm Osteo, weil der Konzern neu in die Traumatologie einsteigen wollte.

Wie kam es danach zur sprunghaften Erweiterung von Stryker in Selzach?

1998 übernahm Stryker von Pfizer das ebenfalls in der Traumatologie tätige Unternehmen Howmedica. Dieses war wesentlich grösser als Osteo, das innerhalb von Stryker der kleinste Teilbetrieb war. Aus diesem Grunde wurde dort die Prothetik fallengelassen. Hingegen wurde im Werk Selzach die Traumatologie ausgebaut. Innerhalb der Unternehmensgruppe wurde die Fertigung mehr und mehr in Selzach konzentriert. Diese Sparte macht heute einen grossen Teil der Wertschöpfung vor Ort aus.

Weshalb hat sich Stryker für Selzach als konzerninternes Zentrum der Traumatologie entschieden? Die Produkte aus Selzach gehören zur Traumalinie, wo Osteo Erfahrung, Know-how und den entsprechenden Talentlevel hatte. Die Leute in unserer Region haben Präzision im Blut. Das präzise Denken und Arbeiten am Jurasüdfuss basiert auf der Mikromechanik der hier ansässigen Maschinen- und Uhrenindustrie. Die Anforderungen sind in jüngster Zeit noch gestiegen, da wir Knochenplatten mit Knochenschrauben auf absolutem Hightech-Niveau produzieren. Das Präzisions- und Qualitätsbewusstsein schafft zudem eine für die Region typische Mentalität: Vorbildliche Arbeitseinstellung, hohe Motivation, Pünktlichkeit, ausgeprägtes Commitment, Engagement und Identifikation mit dem Betrieb.

Ein weiterer Erfolgsfaktor ist das duale Ausbildungssystem, das praxisnahes Knowhow generiert. Zusammen mit den Universitäten und Fachhochschulen ist in der Schweiz eine geballte Ladung an Wissen vorhanden. Auch bei Stryker Selzach selbst werden Aus- und Weiterbildung ausgebaut, nicht zuletzt, um zumindest partiell dem Fachkräftemangel zu begegnen. 
Kann auf Wissensebene von einem Medtech-Cluster am Jurasüdfuss gesprochen werden? Alle grossen Firmen gehen dorthin, wo Know-how bereits vorhanden ist. Daraus entstehen Konkurrenzsituationen und eine gegenseitige Stimulierung, was wiederum zu Bestleistungen antreibt. Das sind die positiven Auswirkungen eines Clusters.

Im Interesse der Medtech-Region «Jurasüdfuss» habe ich bei der Ansiedlung von Biogen in Luterbach mitgeholfen, indem ich Tipps für den Aufbau einer Firma in der Region weitergab. Hilfreich sind in diesem Zusammenhang die positiven Kontakte zu Behörden und Wirtschaftsförderung. So konnten wir für unseren Neubau Land benutzen, das vorher Landwirtschaftszone war.

Gibt es weitere Erfolgsfaktoren für die regionale Medizintechnik?

Wir haben in der Schweiz stabile politische Rahmenbedingungen, keine mächtigen Gewerkschaften, eine gute Zusammenarbeit zwischen Politik und Wirtschaft.

Ein US-Konzern geht in keine Länder mit starken Gewerkschaften, um sich das Leben nicht unnötig zu erschweren.

In der Schweiz haben wir eine konstruktive Verhandlungskultur auf Betriebsebene. Wir sind gegenüber unseren Mitarbeitern fair und versuchen, ihr Verantwortungsbewusstsein zu fördern. Aus diesem Grunde hatten wir noch nie gewerkschaftliche Zwischenfälle irgendwelcher Art. Wir pflegen eine betriebsinterne Sozialpartnerschaft, fordern und fördern unsere Mitarbeiter. Konzernweit gilt die Devise, dass unsere Mitarbeiter ihre Talente entfalten können.

Diese Grundhaltung zeigt sich auch in unserer Organisationsstruktur. Wir haben in Selzach flache Hierarchien. Die Produktion erfolgt in flexiblen Fertigungsinseln, was beim Einzelnen ein hohes Mass an Eigenverantwortung erfordert. Wichtig ist für uns zudem das regionale Lieferantennetzwerk, produzieren wir doch nur ungefähr 50 Prozent der Wertschöpfung inhouse. Wir arbeiten intensiv mit externen Partnern und schaffen so ein breiteres Medtech-Umfeld.

Die Gespräche mit Bruno Bohlhalter, Jesus Fernandez, Urs Graber und Wolfgang Hafner bestanden aus punktuellen Informationen und wurden deshalb nicht aufgezeichnet. Das Gespräch mit Thomas Straumann ist in Kapitel 8 dieser Publikation wiedergegeben. 
Wer von der Medizintechnik am Jurasüdfuss spricht, denkt an Konzerne der Unfallchirurgie und Orthopädie. Die Erfolgsgeschichte von Unternehmen wie Mathys, Stryker, DePuy Synthes beginnt 1958 mit der Begegnung des Chirurgen Maurice E.Müller und des Mechanikers und Konstrukteurs Robert Mathys. Die von Standesdünkeln freie Zusammenarbeit dieser beiden genialen Fachleute löste die Entwicklung eines neuen Industriezweigs aus, zu dem heute auch Ypsomed gehört, der Weltmarktführer für Injektionssysteme.
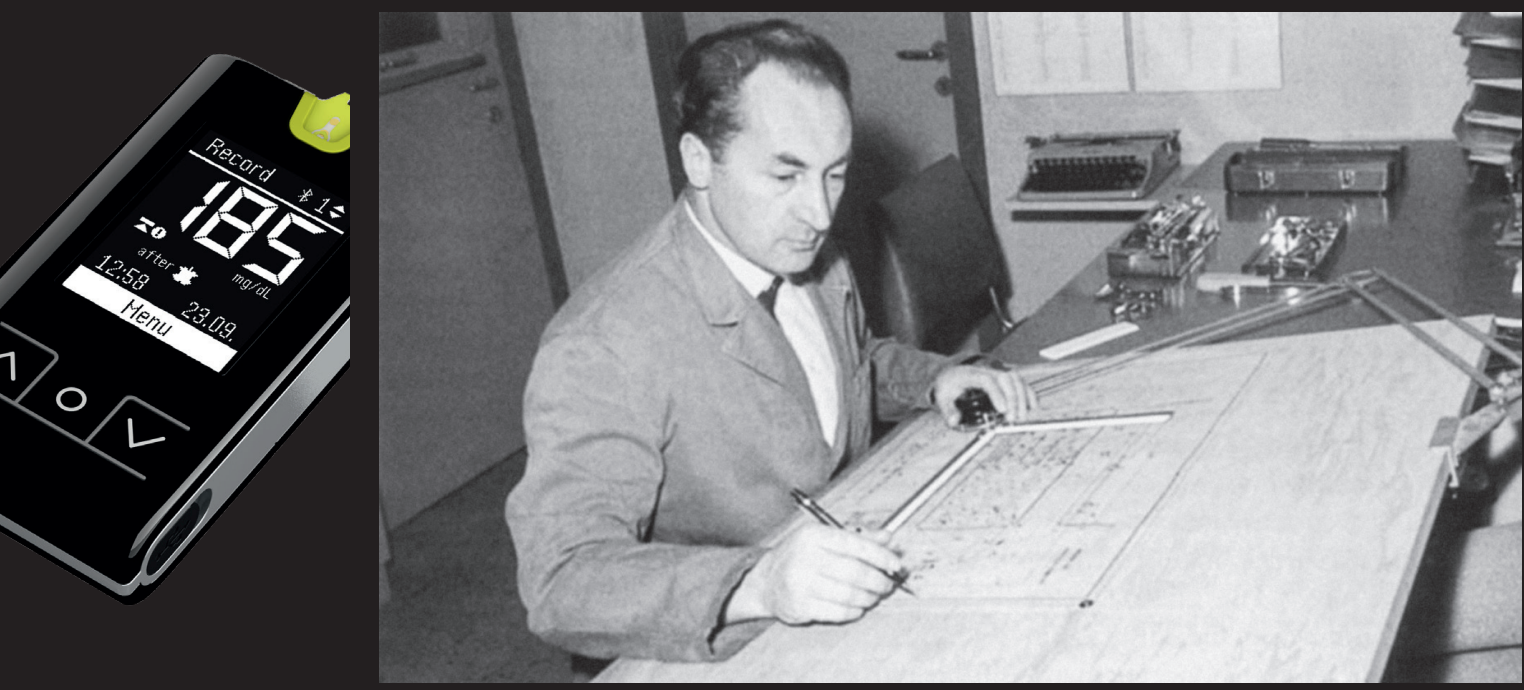

Dieses Buch zeigt konkret und verständlich auf, weshalb die Region zwischen Grenchen und Solothurn zu einem Hotspot der Medizintechnik geworden und bis heute geblieben ist.

Viktor Moser (geboren 1948) studierte Geschichte und Soziologie, war zwischen 1974 und 1988 Bildungsverantwortlicher des Schweizerischen Gewerkschaftsbundes und später Fachmann für Schulung, Teamsupervision und Einzelcoaching im Auftrag von Bund, Kantonen und privaten Unternehmen sowie von sozialen und kulturellen Institutionen. Er war Redaktor einer Fachzeitschrift für Berufsbildung, Berufsberatung und Arbeitsmarkt. 2019 schloss er einen Lehrgang für angewandte Geschichte mit dem Masterdiplom ab. 